\title{
A Fyn - specific biosensor reveals localized, pulsatile kinase activity and spatially regulated signaling crosstalk
}

Ananya Mukherjee*,1,4, Randhir Singh*,1, Sreeram Udayan ${ }^{1}$, Sayan Biswas ${ }^{1}$, Purushotham Pothula ${ }^{2}$, Saumya Manmadhan ${ }^{1}$, Geen ${ }^{1}$, Shilpa Dilip Kumar ${ }^{1}$, Ranabir Das $^{2}$, Balaji M. Rao ${ }^{3 凶}$, and Akash Gulyani ${ }^{1 凶}$

\section{Authors Affiliations}

1. Institute for Stem Cell Science and Regenerative Medicine, Bangalore, India

2. National Centre for Biological Sciences, Bangalore, India

3. North Carolina State University, Raleigh, North Carolina, USA

4. SASTRA University, Thanjavur, Tamil Nadu, India

*; Equal contribution

$\triangle$ To whom correspondence should be addressed:

Email: akashg@instem.res.in

bmrao@ncsu.edu

Phone: +918061948230 


\begin{abstract}
Cell behavior is controlled through spatio-temporally localized protein activity. Despite unique and often contradictory roles played by Src-family-kinases (SFKs) in regulating cell physiology, activity patterns of individual SFKs have remained elusive. Here, we report a biosensor for specifically visualizing active conformation of SFK-Fyn in live cells. We deployed combinatorial library screening to isolate a binding-protein (F29) targeting activated Fyn. Nuclear-magnetic-resonance (NMR) analysis provides the structural basis of F29 specificity for Fyn over homologous SFKs. Using F29, we engineered a sensitive, minimally-perturbing fluorescence-resonance-energy-transfer (FRET) biosensor (FynSensor) that reveals cellular Fyn activity to be spatially localized, pulsatile and sensitive to adhesion/integrin signaling. Strikingly, growth factor stimulation further enhanced Fyn activity in pre-activated intracellular zones. However, inhibition of focal-adhesion-kinase activity not only attenuates Fyn activity, but abolishes growth-factor modulation. FynSensor imaging uncovers spatiallyorganized, sensitized signaling clusters, direct crosstalk between integrin and growthfactor-signaling, and clarifies how compartmentalized Src-kinase activity may drive cell fate.
\end{abstract}


Regulation of cell fate and behavior is achieved through complex and interconnected signaling networks acting in concert ${ }^{1-3}$. For precise signaling, activities of key signaling proteins are tightly compartmentalized in the cell, both spatially and temporally ${ }^{4-6}$. Especially important to cellular controls are signaling nodes that function downstream of multiple receptors classes and help in signal integration. Src family kinases (SFKs) are such key signaling nodes; activated by cell adhesion receptors, integrins, receptor tyrosine kinases (RTKs, including growth factor receptors) and G-coupled protein receptors (GPCR) among others ${ }^{7-11}$. Src-kinases critically influence cell fate; regulating cell shape, migration and adhesion, survival and growth, stemness and differentiation making them important therapeutic targets in multiple diseases ${ }^{12-20}$. Despite their importance, intracellular activity patterns of individual Src family kinases with spatial and temporal precision are still unclear. Visualization of active kinases in cells is essential to understand how Src kinases integrate signals and regulate multiple, and sometimes opposing processes, with fidelity and precision.

Currently available fluorescent SFK sensors have severe limitations. Most available biosensors, especially genetically-encoded one, do not directly report the intracellular distribution of active kinases since they rely on detecting the phosphorylation of "pseudosubstrate" peptide ${ }^{21-25}$. As a result, biosensor readout - extent of phosphorylation of 'sensor' peptides -can be affected by the activities of kinases as well as cellular phosphatases. Confounding readouts further, it has been reported that sensors can get trapped in an "ON" state owing to strong intramolecular interactions between phosphorylated substrate peptides and their respective recognition motifs ${ }^{6,26}$; and therefore are unable to report the turning OFF of kinase activity. Another major drawback of current sensors is that multiple kinases, especially closely related ones, can phosphorylate these substrate-based sensors in a promiscuous manner leading to a lack of specificity.

Study of SFK activity is especially complicated by the presence of multiple closely related Src family members, including three ubiquitously expressed kinases c-Src, cYes and Fyn. While there is some functional redundancy, individual SFKs also perform critical, exclusive roles in the cell ${ }^{20,27-32}$. Therefore, despite decades of study, it is not always clear which kinase(s) is activated in a given cellular context? In this regard, fluorescent biosensors that report the activation of individual Src kinases in live cells would be extremely valuable in clarifying kinase activity and function. However, such specific biosensors for individual Src kinases are still limited ${ }^{5,33,34}$, with current sensors generally giving only a pan-SFK readout with limited spatial and temporal resolution.

Here we present a fluorescent biosensor specific for the SFK-Fyn. Fyn, is ubiquitously expressed and regulates cell migration, epithelial to mesenchymal transition (EMT), cancer metastasis, immune-response, axonal guidance and patterning, and synaptic function $^{13,35-39}$. Interestingly, among several SFKs implicated, recent evidence shows Fyn may specifically control EMT and metastatic progression ${ }^{13}$. Overall, Fyn is a critical player in multiple cell/tissue types, integrating signaling through multiple receptor classes ${ }^{32,40,41}$ and controlling cell fate. However, there is currently no tool, including specific antibodies, to visualize Fyn activity in cells. As a consequence, there is no information on intracellular Fyn dynamics in live cells. A Fyn biosensor would bridge this gap and offer a method to address Fyn functional complexity as a key signaling node. 
Generation of biosensors is often limited by a lack of naturally available binders that can detect active states of proteins in real-time. To address this, we have developed a strategy of using artificially engineered binders that recognize the active form of target proteins in live cells ${ }^{5}$. Like other SFKs ${ }^{42-46}$, Fyn is maintained in an inactive or closed form through multiple intramolecular interactions ${ }^{11}$. Activation of the kinase involves disruption of these interactions leading to an open conformation, with more exposed surfaces $^{5,47}$. Taking advantage of this, we screened a combinatorial protein library to isolate a binding protein (F29) that specifically binds to a region of Fyn (SH3 domain) that is more exposed in the open conformation. For screening, we chose a library generated by mutagenizing the Sso7d protein - a highly stable protein derived from the hyperthermophilic archaeon Sulfolobus solfataricus. We have previously shown that Sso7d mutant-libraries can be used to isolate binding proteins for a wide range of targets $^{48-51}$. Also, since Sso7d has no known interactions with mammalian intracellular proteins, we reasoned that Sso7d would serve as an ideal "inert" scaffold for generating an intracellular biosensor. Our analysis shows that F29 indeed binds specifically to Fyn, with little to no cross-reactivity with other SFKs. Nuclear magnetic resonance (NMR) spectroscopy analysis of F29 in complex with the target reveals the structural basis of the specificity of F29 binding to Fyn.

An efficient readout of the molecular recognition of the target by the binding protein is central to constructing an effective biosensor. Here, we used our new binder/F29 to construct a genetically encoded Fyn biosensor (FynSensor) based on fluorescence resonance energy transfer (FRET). FynSensor is robust, sensitive and faithfully reports Fyn activation and regulation. We also show how FynSensor expression in cells is minimally perturbing, with no measurable changes in either cellular morphodynamics or downstream signaling. Significantly, FynSensor enables direct visualization of active Fyn in live cells, revealing Fyn activation to be spatially compartmentalized, polarized and pulsatile. To the best of our knowledge, this is the first reported visualization of activated Fyn in live cells; notably, unavailability of suitable antibodies currently precludes even routine detection -of active Fyn. Strikingly, FynSensor imaging shows the presence of spatially localized signaling clusters sensitive to integrin and growth factor signaling; and reveals how growth factor response in cells is influenced by integrin-dependent protein activity. More broadly, FynSensor shows how spatially compartmentalized activation of key signaling proteins may lead to efficient signal integration and precise control of cell physiology. Further, our results provide a framework for systematic development of intracellular biosensors for specific Srcfamily and other kinases in general.

\section{Results}

\section{Screening yields binder specific for active Fyn}

Specifically recognizing active Fyn is critical for constructing an intracellular sensor. Activation of SFKs is accompanied by large conformational changes; the active kinase adopts a more open conformation with significantly reduced intramolecular interactions 11. Specifically, prior evidence ${ }^{5,47}$ suggests that the $\mathrm{SH} 3$ domain in SFKs is more accessible in the active conformation relative to the closed, inactive state (Fig. 1a). Therefore, a binding protein targeting the SH3 domain of Fyn can be expected to preferentially bind the active form of the kinase ${ }^{5}$. Such an active state binder can then be engineered as a fluorescent biosensor for reporting intracellular Fyn activation (Fig. 
1a, b). Accordingly, we sought to generate a binding protein specific for Fyn $\mathrm{SH} 3$ domain. Towards this end, we screened a yeast surface display library of Sso7d mutants (library generated by randomizing ten Sso7d residues), using magnetic screening and fluorescence associated cell sorting (FACS), adapting methods described previously $^{49,52}$ (Supplementary Fig. 1a, b). Stringent negative selection steps were included to eliminate binders that exhibited cross-reactivity to the SH3 domains of seven other SFKs, specifically c-Src, c-Yes, Fgr, Blk, Lck, Lyn, and Hck. The pool of yeast cells obtained after magnetic selection were further subjected to multiple rounds of FACS to enrich Fyn SH3-binding yeast cells (Supplementary Fig. 1c). Single clones were then picked and assessed for specific binding to Fyn-SH3. One such clone (referred to as F29 hereafter, see Fig. 1d for sequence and comparison with Sso7d) showed specific binding to Fyn-SH3 domain, with little or no cross-reactivity with SH3 domains from the other highly homologous SFKs mentioned above (Fig. 1c \& Supplementary Fig. 2).

We further examined if F29 retained binding to Fyn-SH3 when removed from the context of the yeast cell surface. F29 fused with glutathione-S-transferase(GST-F29) was recombinantly expressed and purified, and immobilized on glutathione sepharose beads. Immobilized GST-F29 could pull down 6xHistidine (6xHis) -tagged Fyn-SH3, as detected by immunoblotting using an anti-6xHis antibody. In contrast, no signal was seen when the Fyn SH3 pulldown experiment was done with GST-saturated beads, or when the SH3 domain of Src was pulled down using GST-F29 beads (Supplementary Fig. 3, Fig. 1e). Taken together these results confirm that F29 binds specifically to the Fyn-SH3. We further used pulldown experiments to estimate the binding affinity $\left(\mathrm{K}_{\mathrm{D}}\right)$ of the interaction between F29 and Fyn-SH3. Briefly, immobilized GST-F29 was incubated with varying concentrations of Fyn-SH3, and the amount of bead-bound FynSH3 was quantified. Upon fitting the data to a monovalent binding isotherm, the apparent $\mathrm{K}_{\mathrm{D}}$ of the F29-Fyn-SH3 interaction was estimated as $60 \pm 16 \mathrm{nM}$ (Fig. 1f).

\section{Molecular basis of specific Fyn recognition by F29}

F29 specifically binds Fyn SH3 while showing little or no cross-reactivity to SH3 domains of other highly homologous SFKs. To investigate the molecular basis of the specificity of F29 binding to Fyn-SH3, we used NMR spectroscopy to determine the structure of F29 in complex with Fyn-SH3. For this, we first analyzed the structure of isolated F29. The ${ }^{15} \mathrm{~N}$-edited Heteronuclear Single Quantum Correlation (HSQC) spectrum of purified F29 (isolated from $E$. coli grown in ${ }^{13} \mathrm{C},{ }^{15} \mathrm{~N}$-labeled media) showed well-dispersed peaks, indicating proper folding of the protein despite extensive mutagenesis of the Sso7d scaffold (Fig. 2b). Complete chemical shift assignments of the backbone and side chain atoms using a series of 2D and 3D NMR experiments were then used to calculate a structure by the CS-ROSSETTA algorithm ${ }^{53}$. The structure of F29 superimposed well with Sso7d (Supplementary Fig. 4a) indicating a conservation of the Sso7d fold in the binder.

To obtain the structure of Fyn-SH3:F29 complex, ${ }^{15} \mathrm{~N}$-edited HSQC spectra of ${ }^{15} \mathrm{~N}$ labeled free Fyn SH3 domain (residues 87 to 139 of h-Fyn) as well as in complex with F29 (unlabeled) were acquired. The differences between chemical shifts in the free and bound Fyn-SH3 provided the Chemical Shift Perturbations (CSPs) in Fyn-SH3 due to F29 binding (Fig. 2a, c). High CSPs point to specific Fyn SH3 residues likely present at the binding interface in the complex with F29 (Fig. 2c). Similarly, the CSPs in ${ }^{15} \mathrm{~N}$ - 
labeled F29 upon binding Fyn-SH3 were also obtained (Fig 2b, d); these values help identify the F29 binding interface in the complex. A 3D ${ }^{15} \mathrm{~N}$-edited Nuclear Overhauser Effect Spectroscopy (NOESY) experiment, performed on a complex of ${ }^{15} \mathrm{~N},{ }^{2} \mathrm{H}$-labelled Fyn-SH3 and unlabeled F29, led to the unambiguous assignment of $\sim 7$ intermolecular NOEs from the spectra. These NOEs helped identify through-space interfacial interactions between residues. Using the structure of Fyn-SH3 (pdb: 3UA6), the structure of F29 obtained above, and the CSPs as ambiguous restraints, and NOEs as unambiguous restraints, the structure of Fyn-SH3:F29 complex was solved by Haddock $^{54,55}$.

After analysis, the most populated cluster had 200 structures (Supplementary Table 3) and the lowest energy structure and its surface representation are shown in Supplementary Fig. $4 \mathbf{b} \&$ Fig. 2e, f. The contact residues at the interface of the complex are shown in Fig. 3a, b. At the binding interface several non-covalent interactions including hydrophobic-aromatic, hydrogen bonds and salt bridges can be observed (Supplementary Table 4). Eight of the ten randomized residues as well as five originals (SSo7d scaffold) amino acids of F29 show interactions with twelve amino acids of Fyn SH3 (Fig. 3c). Analysis of the binding interface reveals that residues Phe22, Thr24, Tyr26, and Phe31, 32, 43 of F29 show multiple interactions with the Fyn SH3 domain, with SH3 residues Tyr137, Asn136, Tyr132, Trp119, Arg96 and Tyr91 featuring prominently in these interactions. Interestingly, Lys9 (an original Sso7d residue) and Arg33 (randomized residue) on the F29 binder showed exclusive salt bridge interactions with Asp118 and Asp99 of Fyn SH3 domain, respectively (Fig. 3c).

SH3 domains naturally interact with proteins with poly-proline motifs ${ }^{56}$. To gain insight into how F29 binds and specifically recognizes Fyn SH3, we compared the F29-Fyn SH3 complex with structures of FYN-SH3 bound to poly-Proline peptides (pdb: 4EIK and pdb: 3UA7) (Fig. 3d, e). The buried surface area of F29:Fyn SH3 complex is much larger $\left(800 \AA^{2}, \Delta \mathrm{G}=-7 \mathrm{kcal} / \mathrm{mol}\right)$ compared to that of the poly-Pro:Fyn SH3 complex (pdb: 3UA7, 330 $\AA^{2}, \Delta \mathrm{G}=-5 \mathrm{kcal} / \mathrm{mol}$ ). The number of contacts is also considerably larger in the F29 ( 84 contacts) versus the poly-Proline (40 contacts). Moreover, the orientation of the poly-Prolines at the interface differs from the orientation of the F29 interfacial beta-sheet by $\sim 30^{\circ}$. Hence, although the F29 interface overlaps with the polyProline interface, it is significantly distinct in terms of contacts, surface area and orientation.

We then used site-directed mutagenesis to examine contributions of key interfacial residues to F29-Fyn SH3 binding. A salt bridge between the residue R33 of F29 and D99 of Fyn SH3 appears prominent. Indeed, the introduction of an R33A mutation in F29 resulted in reduced binding to Fyn-SH3 domain, as evidenced by pull-down analysis (Supplementary Fig. 5a). These results confirm that R33 in F29 and D99 in Fyn-SH3 contribute substantially to the F29-Fyn-SH3 binding interaction. Interestingly, a Pro41Ala mutant of F29 also showed reduced binding to Fyn-SH3 (Supplementary Fig. 5a). Although the NMR analyses do not show Pro41 to form direct interfacial contacts with $\mathrm{SH} 3$, the reduced binding of this mutant may be attributed to perturbation of local structure. Proline41 forms a critical hinge preceding $\beta 3$ strand (Fig. 3b); mutation of the proline likely disrupts the structure the $\beta$-sheet that contains multiple key binding contacts with Fyn-SH3. 
These structural analyses offer considerable insight into the molecular basis for the specificity of F29 binding to Fyn-SH3 over highly homologous SH3 domains of other SFKs. The binding interface in the Fyn-SH3-F29 complex reveals multiple interactions (Fig. 3b, c). Notably, while almost all the interacting residues in Fyn-SH3 are conserved in other SFKs, D99 in Fyn-SH3 that forms a salt bridge is unique to ubiquitously expressed Fyn as well as Lyn and Fgr (Supplementary Fig. 5c). Further, the pool of Fyn-SH3 binders isolated from the combinatorial Sso7d library after multiple rounds of FACS showed cross-reactivity with the SH3 domain from Yes (Yes-SH3), but not other SFKs (Supplementary Fig. 1c). Therefore, we hypothesized that D99 in FynSH3 is a key determinant of binding specificity of F29 for Fyn-SH3. To test this hypothesis, we generated a mutant version of the SH3 domain from Src (Src-SH3) wherein the native threonine was replaced with aspartic acid (T99D). Strikingly, T99D Src-SH3, but not wild-type Src-SH3, showed detectable binding to F29 in pulldown assays (Supplementary Fig. 5b). These results confirm that D99 in Fyn-SH3 is a key determinant of the binding specificity of F29 for Fyn over other SFKs. Nevertheless, specificity of F29 for Fyn-SH3 over Yes-SH3 (Fig. 1c, e), which contains the E99 residue, underscores the point that binding specificity results from a unique combination of multiple interactions at the binding interface.

\section{F29 enables construction of FynSensor, a FRET biosensor for active Fyn}

F29 efficiently and specifically binds Fyn SH3 in-vitro. We then examined if F29 is able to recognize full-length, cellular Fyn and if it preferentially binds the active form of Fyn. To test this, either WT Fyn kinase or the CA mutant ${ }^{57}$ (active and open conformation) was exogenously expressed in human embryonic kidney (HEK-293T) cells and the cell lysate treated with immobilized GST-F29. Fyn pulldown and immunoblotting showed that the CA mutant was more efficiently pulled down by F29 as compared to the WT Fyn kinase (Fig. 4a). On the contrary, GST-alone control beads showed no significant pulldown of either WT or CA Fyn. These data indicate that F29 preferentially binds active Fyn; the SH3 domain targeted by F29 is more accessible in the CA mutant compared to the WT Fyn kinase. Further, similar GST-pulldown and immunoblot analysis showed that immobilized F29 (GST-F29), but not GST-alone control, was able to bind and pull-down endogenous or native Fyn from cell lysates (Fig.4b). These results confirm efficient binding of F29 to cellular Fyn.

Taken together, our results so far show F29 to be a promising candidate for generating an intracellular biosensor for active Fyn. Accordingly, we conceptualized a biosensor design based on FRET wherein the binding of F29 to active Fyn would result in an increase in FRET between suitably placed donor and acceptor fluorophores (Fig. 4c). To construct a genetically encoded biosensor, Fyn was labeled with a FRET donor fluorescent protein (mCerulean) while F29 was tagged with a FRET acceptor protein (mVenus). While F29 already shows high specificity to Fyn, our design further ensures that FRET signal can arise only from labeled F29 binding to labeled active Fyn. For generating the fluorescently labeled Fyn, we chose to introduce mCerulean (FRET donor) between the unique (UD) and the SH3 domains of full-length Fyn kinase ${ }^{33}$ (Fig. 4c). The choice of this insertion position is designed to increase the probability of efficient FRET when acceptor-labeled F29 binds the exposed SH3 domain in active Fyn. Another critical design consideration was to minimize any perturbation caused due to mCerulean insertion into Fyn. To this end, we selected a flexible poly-(glycineserine) peptide linker ${ }^{58}$ to insert mCerulean within Fyn (also see method and 
Supplementary Fig. 6 for details on molecular engineering). We then examined the suitability of the designed biosensor constructs for cellular imaging. Immunoblotting analysis on cell lysates showed robust intracellular expression of full-length mCerulean-Fyn fusion proteins (hereby referred to as 'mCer-Fyn'), confirming that the fusion proteins remain intact and resistant to proteolysis in cells (Supplementary Fig. 7a, i). Further, mCer-Fyn fusions expressed in cells showed expected fluorescence; with spectra from cells closely resembling unmodified mCerulean (Supplementary Fig. 7c). Additionally, the mVenus-F29 fusion biosensor protein also expressed well, was resistant to proteolysis (Supplementary Fig. 8a) and showed the expected fluorescent signatures resembling unmodified mVenus (Supplementary Fig. 8b). Importantly, we then tested if labeled Fyn kinase retains activity and is regulated appropriately. Src family kinases possess autocatalytic activity; a tyrosine residue (Y420 in h-Fyn) in the activation loop is phosphorylated when the protein gets activated $^{59,60}$ Significantly, immunoblot analysis showed that mCer-Fyn retains autocatalytic activity similar to the wild-type unmodified protein in cells. Further quantification of Fyn Y-420 autophosphorylation levels, show no significant differences between mCer-Fyn and unlabeled wt-Fyn (Supplementary Fig. 7a, b). Importantly, these data show that mCerulean insertion is minimally disruptive to kinase activity and that the engineered kinase appears to be regulated similar to unmodified Fyn.

To further test if our Fyn fusion (mCer-Fyn) is functional and can accurately reflect cellular Fyn dynamics, we examined if mCer-Fyn can modulate downstream signaling and functionally replace endogenous, wild-type Fyn. For this we carefully assayed Fyn effects on downstream signaling, as measured through extracellular receptor kinase (ERK) phosphorylation and performed Fyn knock-down rescue in cells, comparing both unlabeled as well as Fyn-fusion (mCer-Fyn) constructs. We first established that a RNA-i knockdown of cellular Fyn protein in HEK293T cells (Supplementary Figure 9), significantly attenuates the levels of ERK phosphorylation in cells. This is consistent with earlier reports documenting Fyn's role in modulating ERK activity and downstream signaling ${ }^{61}$. Notably, expressing either mCer-Fyn or wt, unlabeled Fyn in 'Fyn-knockdown' cells significantly rescues this reduction in ERK phosphorylation. Further, the extent of increase in this ERK phosphorylation, mediated through ectopic Fyn expression in 'Fyn-knockdown' cells, was observed to be the same for wild-type, unlabeled Fyn as well as mCer-Fyn (Supplementary Figure 10). These results unequivocally demonstrate that our labeled Fyn (mCer-Fyn) is regulated appropriately, 'signaling competent' and can functionally replace native, untagged Fyn; thereby making it suitable for faithfully reporting cellular Fyn dynamics.

Biosensor variants were then tested for efficacy in reporting intracellular Fyn activity. For this, we expressed biosensor constructs in HEK-293T cells and recorded fluorescence spectra from live cells (see methods). When cells co-expressing FRET donor (mCer-Fyn) and FRET acceptor (mVenus-F29) were excited at $435 \mathrm{~nm}$ (mCerulean/donor excitation), emission peaks were seen at $\sim 475 \mathrm{~nm}$ (mCerulean) as well as at $\sim 525 \mathrm{~nm}$ corresponding to the peak emission wavelength of the acceptor fluorophore (mVenus) (Fig. 4d). Appearance of acceptor emission (mVenus, $~ 525 \mathrm{~nm}$ ) on donor excitation $(435 \mathrm{~nm}$ ) clearly shows that FRET occurs between mCer-Fyn and mVenus-F29. This confirms that mVenus-F29 can recognize intracellular Fyn in live cells and this recognition can be reported through a robust FRET signature. We then tested if this FRET signal is sensitive to the relative stoichiometry of the target (Fyn) 
and the binder F29 as would be expected with a binding-induced FRET. Indeed, increasing the amount of intracellular mVenus-F29 while maintaining constant expression of mCer-Fyn, led to a concomitant increase in FRET signal, followed by saturation of this FRET increase. Here, the saturation in FRET signal clearly indicates that even increasing concentrations of F29 binder in cells do not simply cause an inadvertent or artefactual activation of the kinase. Importantly, the FRET signal in cells is specific and dependent on mVenus-F29 binding to mCer-Fyn, since making a single point mutation in F29 abolishes the FRET response. If the non-binding variant of F29 (mVenus-F29-P41A) is co-expressed with mCer-Fyn in cells, little or no FRET is seen, even at higher concentrations of the non-binding control (Fig. 4d left inset). Further, the FRET signal was higher in cells co-expressingmVenus-F29 and constitutively active Fyn, relative to wild-type Fyn (Fig. 4d right inset). This shows that F29 binding and biosensor readout (FRET response) is indeed sensitive to the activation status of Fyn (Fig. 4d).

Fyn kinase has two acylation marks (a myristoyl and a palmitoyl group) that make it preferentially localized to the cell membrane ${ }^{62}$. Therefore, we reasoned that localization of F29 to the cell membrane would increase its proximity to membrane-bound Fyn, resulting in an increase in FRET signal and consequently greater dynamic range of the biosensor. To test this hypothesis, we added a myristoylation('myr') sequence (MGSSKSKPKDPS) to the F29 binder $^{63}$. Indeed, addition of a myristoylation signal led to a substantial increase in FRET signal (Fig. 4e). To further test the fidelity of this enhanced FRET response observed with myristoylated-F29, we tested the non-binding variant (P41A) of the myr-mVenus-F29 binder. Notably, myristoylated non-binding mVenus-F29 P41A showed little or no FRET signal when co-expressed with mCer-Fyn (Fig. 4e). Taken together, these results clearly show that the FRET signal observed with the F29-based Fyn biosensor is indeed due to specific binding of F29 to the active form of Fyn, and not just due to non-specific membrane localization and incidental proximity. The biosensor constructs - mCer-Fyn and myr-mVenus-F29 (referred to as binder) - are collectively referred to as FynSensor hereafter.

Src family kinases, including Fyn are known to strongly regulate cell adhesion as well as the cytoskeleton. Several SFK substrates and interacting partners are reported to be actin binding proteins, regulators of Rho family GTPases and other proteins that help remodel and regulate the actomyosin network ${ }^{3,64-66}$. We therefore reasoned that measuring the cell morphodynamics ${ }^{5,67}$ would be a sensitive way to examine if moderate to low expression of labelled Fyn and the F29 binder significantly and measurably perturb the cell. First, the expression levels of FynSensor constructs labeled Fyn (mCer-Fyn) as well as mVenus-F29 were quantified in U2OS cells (Supplementary Fig. 11). We then quantitatively examined temporal changes in cell area and perimeter of cells expressing either FynSensor (mCer-Fyn:myr-mVenus-F29, DNA ratio 1:2, also see Fig. 4d) or the binder alone or a control construct (myr-taggedmVenus) in the adherent osteosarcoma U2OS cells at the expression levels specified (Supplementary Fig. 11). We find no significant change in cellular morphodynamics as a result of FynSensor expression the binder alone as compared to controls cells (Supplementary Fig. 12). We further confirmed that the expression of Fyn-binder does not perturb the overall intracellular localization of labeled Fyn (Supplementary Fig. $13)$. 
While FynSensor and F29 expression had no effect on cell morphodynamics and Fyn localization, we also examined if FynSensor expression significantly perturbs downstream signaling. For this, we again used the extent of ERK phosphorylation as a sensitive readout of Fyn signaling. We find that neither expression of FynSensor nor binder alone in adherent osteosarcoma U2OS cells under the conditions used has any measurable effect on ERK phosphorylation levels (Supplementary Fig. 11). Similarly, expression of FynSensor constructs (labeled Fyn + binder) in C2C12 mouse myoblasts (Supplementary Figure 14) or Fyn-knockdown HEK-293 cells (Supplementary Fig. 10) did not significantly change levels of p-ERK. Notably, we further confirmed by immunofluorescence analysis that levels and intracellular localization patterns of $p$ ERK are not affected by expression of FynSensor in cells (Supplementary Fig. 15). These data collectively show that FynSensor expression under these specified conditions does not significantly perturb downstream signaling. In light of these data showing little or no cellular perturbation, we have used the same FynSensor expression conditions for all further biosensor imaging studies.

\section{FynSensor reports localized active Fyn in live cells}

Our results show that FynSensor produces a FRET signal upon specific binding of F29 to Fyn-SH3, resulting in a sensitive readout of active intracellular Fyn. We further evaluated FynSensor for its ability to report spatial patterns of active Fyn in single, living cells. To investigate the spatial FRET response generated by FynSensor, we first employed the 'fluorescence recovery after acceptor photobleaching (APB)' method. APB analysis is a robust method of confirming and quantifying FRET in cells ${ }^{68}$, where recovery of donor fluorescence on APB is seen as a strong indicator of proximityinduced FRET. Energy transfer from the donor to the acceptor molecules results in quenching of donor fluorescence, and this quenching is relieved when proximal acceptor molecules undergo photobleaching. Indeed, when adherent osteosarcoma U2OS cells expressing FynSensor were illuminated with increasing doses of $514 \mathrm{~nm}$ laser light (wavelength corresponding to acceptor absorption; mVenus photobleaching), we observed a substantial, dose-dependent recovery of donor (mCerulean) fluorescence. This confirms FRET between mCer-Fyn and mVenus-F29. Significantly, APB-induced recovery of donor fluorescence was greater at cell edges (Fig. 5a, b), suggesting spatial variation in Fyn activity. Notably, in control cells expressing a mutant form of labeled-F29 (P41A), acceptor photobleaching showed no such dose-dependent recovery of donor fluorescence (Fig. 5c). Since the P41A mutant lacks the ability to bind Fyn-SH3, these data show that APB-induced recovery of donor fluorescence and the FRET response are due to F29 binding to target and not due to incidental proximity or optical artifacts. Finally, when cells were treated with a known pharmacological inhibitor of Src family kinase including Fyn, SU6656 ${ }^{69}$, we observed a significant reduction in the amount of donor fluorescence being recovered (Supplementary Fig. 16). This reduction in FRET (F29 binding to Fyn) on inhibitor treatment is interesting and points to allosteric changes in Fyn conformation on inhibitor binding. While ATP-competitive inhibitors like SU6656 directly bind the catalytic domain, they can also stabilize an inactive conformation of Src family kinases and modulate the accessibility of its key regulatory domains to ligands/binding proteins ${ }^{70}$. Therefore, our data (reduced FynSensor FRET) suggests that indeed Fyn likely adopts a closed conformation in response to inhibitor treatment, thereby reducing SH3 domain accessibility/binding to F29. The sensitivity of FynSensor FRET to inhibitor binding in fact suggests that the labeled kinase shows allosteric 
conformational changes similar to those seen with untagged SFKs, further indicating that the biosensor faithfully reports Fyn conformational dynamics. Taken together, these results further confirm that the FynSensor readout is the result of specific binding of F29 binding to Fyn-SH3, and selectively reports the active form of Fyn (Fig. 5b, c and Supplementary Fig. 16). The FynSensor readout also shows hitherto uncharacterized spatial patterns in intracellular active Fyn in live cells, with greater Fyn activity closer to the cells edge.

\section{FynSensor reveals pulsatile Fyn activity and spatially compartmentalized signal integration}

We used FynSensor to examine the spatial and temporal dynamics of Fyn activity, especially in light of the important role played by Fyn as a signaling node functioning downstream of distinct receptors classes. We reasoned that ability to specifically image the active conformation of a single SFK would provide new insight into how signal integration may occur. We first examined Fyn activation dynamics in serum-starved cells plated on fibronectin (FN) that show constitutive protrusion retraction cycles. For imaging activity dynamics, we used ratiometric sensitized emission (normalized acceptor emission due to donor excitation) to quantify FRET signals from the sensor ${ }^{71}$ (Methods). Ratiometric sensitized emission measurements allow facile and rapid imaging of FRET responses in cells with both spatial and temporal resolution.

Sensitized emission (FRET) imaging with FynSensor in FN-plated serum-starved U2OS cells revealed spatially localized Fyn activity, with clear intracellular zones showing higher total FRET $\left(\mathrm{FRET}_{\mathrm{T}}\right)$. Such intracellular zones showing enhanced FynSensor FRET $_{\mathrm{T}}$ were consistently observed in all cells in the data set (Fig. 6a-i). Since all cells spontaneously appear to show regions of differential Fyn activity, we employed quantitative image analysis to probe these patterns of Fyn activity. An automated cell quadrant analysis of intracellular FRET $_{T}$ levels provides clear evidence of differential and compartmentalized Fyn activity. Briefly, every cell imaged was divided into quadrants in an automated manner and FRET response for each quadrant was analyzed over time. These analyses show substantial and significant differences in Fyn activation levels (FynSensor FRET $_{\mathrm{T}}$ ) between intracellular quadrants (designated as 'low' and 'high' FRET quadrants for consistency and comparison) (Fig 6a-ii). Importantly, the spatial patterns of Fyn activation revealed through FynSensor imaging are robust and are unlikely to be an artifact of imaging, since a single point mutation in the binder completely or substantially abolishes the FRET response seen. When mCerFyn is co-expressed with the non-binding P41A mutant of F29, little or no FRET signal is seen in single cells, clearly showing that FynSensor FRET is dependent on F29 binding to active Fyn (Fig. 6b, Supplementary Fig. 17, Supplementary Video 1). Overall, the spontaneous compartmentalizing of Fyn activity was observed in all cells and is a striking finding revealed through FynSensor imaging. (Fig. 6a).

We demonstrate that the spatially enhanced $\mathrm{FRET}_{\mathrm{T}}$ patterns observed are unlikely to be an artifact of an unequal or spatially-constrained intracellular distribution of the F29 binder. Supplementary Fig. 18 shows that while the FynSensor FRET levels are nonuniform and spatio-temporally patterned, the fluorescently labeled F29 (myr-mVenusF29) is homogeneously distributed in cells. While increased recruitment of Fyn may contribute to the establishment of regions of 'high Fyn activity' in serum-starved, FNplated cells, our data analysis shows that differential Fyn activity observed cannot 
solely be ascribed to increased localization of Fyn. Supplementary Fig. 19 a, b show that intracellular regions showing higher $\mathrm{FRET}_{\mathrm{T}}$ also show significantly higher levels of donor-normalized FRET (donor-normalized FRET $=$ FRET $\mathrm{T} / \mathrm{mCer}_{\mathrm{F}}$-Fyn fluorescence), which normalizes the overall activity for protein levels. This is also illustrated (Supplementary Fig. 20 a \& b) by examples of regions that have almost similar levels of the kinase (mCer-Fyn localization as visualized in the donor channel in regions marked 1 and 2) but show significantly different levels of kinase activity (FRET index image, regions 1 and 2), reaffirming the point that increases in FRET signals are just not due to increase in kinase localization.

We surmised that spatially-enhanced Fyn activity seen in serum-starved cells on fibronectin may be caused by differential integrin signaling. To test if this Fyn activation in serum-starved cells, as visualized through FynSensor, is indeed sensitive to integrin signaling, we treated cells with an inhibitor of focal adhesion kinase (FAK), PF-562271 $1^{72,73}$ and examined FynSensor response (Fig. 6c- i, Supplementary Video 2). Focal adhesion kinase (FAK) is a critical transducer of integrin signaling and is known to partner SFKs in mediating cellular responses ${ }^{74,75}$. Strikingly, when serumstarved, FN-plated U2OS cells were treated with PF-562271, there was an immediate and drastic reduction in the FynSensor FRET response (Fig. 6 c ii-iv)). This reduction in activity is accompanied by a substantial dampening of the temporal changes seen in levels of active Fyn in cells (see below). These results clearly demonstrate that localized Fyn activity, reported through FynSensor, is sensitive to focal adhesion kinase activity; and support the model that compartmentalized Fyn activity arises through differential integrin signaling in serum-starved adherent cells.

To confirm that these spatial patterns of Fyn activity are not just limited to any one specific cell-type, we performed FynSensor imaging in mouse C2C12 myoblasts apart from U2OS osteosarcoma cells. Notably, FynSensor again shows Fyn activity to be spatially patterned in the serum-starved $\mathrm{C} 2 \mathrm{C} 12$ myoblast cells (Fig. 7a-i, ii). Quantitative quadrant analysis confirms differential Fyn activity $\left(\right.$ FRET $\left._{\mathrm{T}}\right)$ in distinct intracellular zones. (Fig. 7a-ii). To assess if cells expressing near-endogenous levels of Fyn kinase also show similar spatial-activation patterns, we performed imaging experiments in HEK-293T cells where the endogenous Fyn has been depleted through RNA-i and then complemented with FynSensor (Supplementary Fig. 21). Biosensor imaging in these HEK cells indeed showed activation patterns very similar to those previously observed in U2OS and C2C12 cells (Fig. 7b). The observation of similar, spatially-enhanced patterns of Fyn activity in these very distinct cell types shows that FynSensor responses are highly robust and is suggestive of conserved spatiallymodulated signaling mechanisms.

To further confirm that the conserved patterns of Fyn activity are not impacted by changes in FynSensor expression levels, we systematically examined FynSensor FRET profiles as a function of expression. In all three cell types examined, the spatial patterns remain conserved independent of the precise levels of labeled Fyn (m-Cer-Fyn) as well as the binder (m-Venus-F29) (Fig. 7c and Supplementary 22).

Another consistent and striking feature revealed by FynSensor is the temporal bursts of Fyn activity. FRET $_{\mathrm{T}}$ levels show clear oscillations over time in serum-starved cells plated on FN and imaged (Fig. 8a, Supplementary Video 3). Interestingly, these oscillations in Fyn activity revealed through FynSensor imaging are seen in multiple 
cell types and appears to be a conserved feature of Fyn activation dynamics. FRET $\mathrm{T}$ profiles show temporal oscillations in C2C12 myoblast (Fig. 8b), Fyn-KD HEK-293T (Fig. 8c) as well as U2OS cells (Fig. 8a) expressing FynSensor. We also tested if these Fyn activity pulses arise due to any sudden changes in kinase localization. Supplementary Fig. 19c clearly shows that along with the FynSensor FRET $_{\mathrm{T}}$ profiles, even the donor/mCerulean normalized FRET levels show pulsatile behavior. This shows that pulses observed are not due to fluctuations in Fyn concentration levels but arise due to direct and rapid regulation/modulation of Fyn activity (Supplementary Fig. 19d). Since the pulsatile nature of Fyn activity was found to be conserved across cell types, we specifically examined the duration/frequency of these pulses in U2OS, C2C12 and HEK-Fyn KD cells. Fig. 8d shows the mean of the dominant time-period of FynSensor FRET pulses in all three cell-types, determined through power spectrum density (PSD) analysis performed on the FRET $_{\mathrm{T}}$ time-traces. The PSD analysis reveals the mean of dominant time-period to be $\sim 3.5 \mathrm{~min}$ in three very distinct cell types, again highlighting a conserved feature of Fyn activity dynamics. There is increasing evidence to suggest that signaling modules, including growth factor responses in cells may show pulsatile behavior ${ }^{76-78}$. Bursts of activity are expected to bear signatures of complex positive and negative (inhibitory) feedback loops that are integral part of growth factor and other signaling modules ${ }^{79-81}$. Interestingly, temporal oscillations are highly pronounced in regions of spatially-enhanced Fyn activity as can be seen by plotting FynSensor FRET $_{\mathrm{T}}$ against time as well as distance from the cell-edge. The timedistance 3-D plot of Fyn activity shows bursts of activity in a tight intracellular zone proximal to the edge. (Fig. 8e-i, ii)

Fyn kinase signals downstream of multiple receptor classes, including integrin and receptor tyrosine kinases (RTKs/Growth factor receptors). Based on prior evidence ${ }^{82-}$ ${ }^{85}$, we hypothesized that Fyn, functioning as a signaling node may be involved in dynamically integrating signals downstream of integrins and RTKs ${ }^{86-88}$. We used the FynSensor to investigate this putative signal integration. For this, we examined the effect of platelet-derived growth factor (PDGF) on FN-plated, serum-starved U2OS cells and visualized active Fyn using FynSensor FRET. As discussed earlier, serumstarved FN-plated cells already showed spatially enhanced and temporally regulated Fyn activity, consistent with constitutive cell polarization and Fyn activation downstream of spatially regulated integrin signaling (Fig. 6a). However, after stimulation with PDGF, FynSensor cells (but not the P41A non-binding control Supplementary Video 1) showed a significant increase in Fyn activity as indicated by enhanced FRET signal (Fig. 9 a). Strikingly, despite a global PDGF stimulation, the increase in Fyn activity observed was spatially localized. It appeared that on stimulation, the FRET $_{\mathrm{T}}$ signal, preferentially increased in regions that already showed higher FRET in un-stimulated cells (Fig. 9a, Supplementary Video 4), resulting in highly compartmentalized Fyn activity patterns.

To quantitatively examine these activity patterns and signal modulation, we again employed automated image analysis. An automated intracellular quadrant analysis confirms differential FynSensor FRET levels across distinct intracellular zones, postPDGF stimulation (Fig. 9 b-i, ii). These data demonstrate that cells remain 'polarized' with respect to Fyn activity despite a global PDGF stimulation. Notably, quadrant image analysis also confirmed a preferential PDGF-induced enhancement of Fyn activity in pre-activated intracellular zones. Fig. 9b-ii shows that PDGF-induced enhancement in FynSensor FRET is greater in intracellular quadrants already showing 


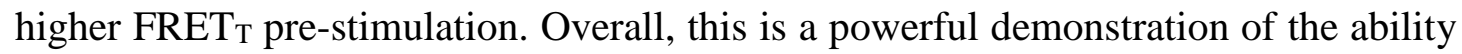
of FynSensor to reveal hitherto unexplored aspects of Fyn signaling dynamics.

FynSensor shows Fyn activity to be compartmentalized even in serum-starved cells. This is likely due to differential integrin signaling, since Fyn activity is significantly attenuated upon FAK inhibition. This spatially constrained Fyn activity could be further increased through growth factor stimulation, with greater enhancement seen in preactivated areas despite a global stimulation. These results provide a direct illustration of dynamic and intracellular localized signaling crosstalk between integrins and growth factor receptors; visualized through the activation (conformational change) of a nonreceptor tyrosine kinase that get activated by each of these receptor classes. While results so far imply that differential integrin signaling appears to spatially restrict the effect of growth factor, we asked if appropriate integrin signaling is required for growth factor mediated modulation of Fyn activity. For this we inhibited focal adhesion kinase activity and specifically tested if growth factor is still able to modulate Fyn activity levels. Interestingly, when FynSensor U2OS expressing cells are treated with PF562271 (FAK inhibitor), not only are the FynSensor FRET levels drastically reduced, the cells become insensitive to growth factor stimulation as measured through Fyn activity. PDGF-stimulation of inhibitor treated cells show little or no change in FynSensor FRET levels (Fig. 9c, Supplementary Video 5). These data indeed confirm a robust, spatio-temporally modulated and functional crosstalk between integrin and growth factor signaling.

FynSensor reveals striking spatio-temporal patterns of Fyn activity. Since Fyn is a key regulator of cell physiology, including cytoskeleton remodeling and adhesion dynamics, we also asked how Fyn activity correlates with cellular morphodynamics. For this we performed quantitative image analysis and measured transient changes in cell area as well as total FRET levels for individual cellular quadrants, over the time course of the experiment (Fig. 10a i-iii). Intriguingly, a plot of fractional area change versus FRET $_{\mathrm{T}}$ levels shows that the two parameters tend to be inversely correlated (Fig. 10b). This is an interesting trend which suggests that intracellular regions showing higher Fyn activity is likely to show reduced overall area changes. This link between Fyn activity and dampened cell membrane oscillations points to a 'poised' cell membrane when/where Fyn is active. Such a notion is consistent with Fyn's role in regulating cell-matrix adhesions and may need to be probed further (Fig 10c). It is remarkable that visualizing the conformational dynamics of a membrane-bound, nonreceptor kinase reveals pulsatile patterns, which can be modulated through integrin and growth-factor signaling.

\section{Discussion}

Direct visualization of protein activity is essential in order to gain a quantitative understanding of dynamic signaling networks that govern cell behavior. Despite the critical roles played by Src family kinases (SFKs) in regulating physiology ${ }^{12-20}$, specific tools/sensors to image activity of individual kinases in live cells and tissues are not available. This is particularly important as individual SFKs can perform overlapping but specific, even seemingly opposing roles, to control cellular output ${ }^{20,27-32}$. Therefore, understanding the specific activity patterns of individual kinases assumes considerable importance. Our work clearly addresses this issue and establishes a platform for developing new biosensors for visualizing activation of individual Src kinases. Using 
combinatorial library screening and protein engineering, we develop a biosensor for the critical SFK, Fyn. This specific biosensor reports the activation dynamics of Fyn in live cells, with no interference from other kinases. Fyn kinase is a major regulator of multiple cellular processes and has emerged as a key player in various disease pathologies ${ }^{16,89-91}$ but this is the first direct visualization of Fyn activity in cells. Interestingly, currently there are no tools for imaging active Fyn, with even specific antibodies not being available.

Our approach is extremely general and can be used for building sensors for other Src family kinases that are critical players in homeostasis and diseases. In this light, our work on addressing the specificity of biosensor binding using NMR structural analysis is likely to be significant. While our targeted screening and binding analysis yields a new binder (F29) that is highly specific for Fyn (Fig. 1), the NMR structure of F29 bound to Fyn SH3 provides fascinating new insights into the molecular basis of specificity (Fig. 2, 3). We identify key interactions that mediate binding and further use this insight to switch specificity of binding (Fig. 3). For instance, our Fyn-specific binder F29 does not or weakly bind Src-SH3 (Fig. 1c, e). However, based on predictions from structural analyses, making just a single residue change in Src SH3 allows it to now bind F29 better (Supplementary Fig. 5b). These findings offer the possibility of generating specific binders targeting various Src family kinases, leading to new biosensors and tools. More broadly, we showcase how targeted screening of combinatorial protein libraries can be deployed to generate genetically-encoded biosensors for visualizing active conformations of signaling proteins. This is a generally applicable strategy for biosensor development. With this method it is possible to develop sensors even for 'difficult' targets, where structural information is limited and conventional tools like antibodies do not exist.

We demonstrate that FynSensor is able to report on the activation status of Fyn in cells. Newly engineered binder F29 binds the active form of Fyn and this binding leads to a FRET response that can be measured in live cells (Fig. 4-10). Our sensor design and extensive control experiments ensures high sensitivity while minimizing perturbation. We have shown that the fluorescently labeled Fyn retains kinase activity, is fully functional and behaves like the unmodified kinase in terms of its regulation, localization and ability to modulate downstream signaling (Supplementary Fig. 7, 10, 13,15). Importantly, the fluorescently labeled Fyn is also able to complement the endogenous Fyn in RNAi-knock-down rescue experiments. For instance, in knockdown-rescue experiments, labeled Fyn is able to rescue downstream ERK phosphorylation similar to untagged Fyn, showing that our tagging of Fyn does not perturb regulation and signaling function. We also demonstrate that under our imaging conditions (low to moderate expression of FynSensor) in two very distinct cell types, biosensor does not perturb either downstream signaling or cellular morphodynamics, reiterating the efficacy of our design (Supplementary Fig. 11). When the non-binding control version of the F29 binder (F29P41A) is used in cells, the FRET response is abolished, showing FRET to be dependent on F29 recognizing activated Fyn (Supplementary Fig. 17). Further, an inhibitor of kinase activity also significantly attenuates FRET response, showing the biosensor readout to reflect kinase activity (Fig. 6b). Overall, we show that the FynSensor response is specific and reflects cellular activity of Fyn.

A significant advantage of FynSensor is that it is highly specific and directly reports on the active conformation of Fyn with high spatial and temporal precision, unlike 
previously reported kinase sensors. This direct visualization of the active form of a single kinase, within a critically important yet complex family of kinases, has led to new insights into signaling dynamics and regulation. FynSensor imaging reveals Fyn activity to be localized and temporally modulated (Fig. 5, 6,7,8,9), with greater activity closer to the cell edge. Further, as serum-starved, fibronectin (FN)-plated cells undergo constitutive cycles of protrusion and retraction, we observe intracellular zones of high Fyn activity and even spontaneous cell polarization measured through levels of active Fyn. Critically, we show that this compartmentalized Fyn activity is dependent on integrin signaling. Inhibition of focal adhesion kinase, a key mediator of integrin signaling, abolishes or greatly attenuates Fyn activity. When FN-plated cells with spatially-localized Fyn activity are treated with platelet-derived growth factor (PDGF), we observe a greater increase in Fyn activity in and around the zones that were already pre-activated. This is indeed remarkable. Despite global PDGF treatment, its effect is highly localized and is dependent on intracellular zones that are established through integrin signaling. Our results suggest a model wherein localized/differential integrin activation in cells undergoing constitutive protrusion-retraction cycles not only helps establish signaling zones/compartments with higher Fyn activity, but also sensitizes these zones to be more responsive to growth factor signaling (Fig. 11). This could be through either preferential localization or pre-sensitization of PDGF-receptors and/or associated signaling components (Fig. 11). Thus compartmentalized integrin signaling is not only maintained but also 'functionally enhanced' through growth factor stimulation. We further show that integrin signaling, not only spatially constraints Fyn activity and subsequent growth factor response, but that appropriate integrin signaling is required for growth factor modulation of Fyn activity. Inhibiting focal adhesion kinase activity not only dramatically attenuates Fyn activity in FN-plated, serumstarved cells, it also renders Fyn insensitive to any further stimulation through PDGF (Fig. 9c).

Our results offer a striking demonstration of spatially localized crosstalk between integrin and growth factor signaling and shows that integrin can localize and regulate the effect of even globally applied growth factors in activating downstream signaling (Fig. 9). This illustrates how by visualizing the dynamic activation of a key signaling node (Fyn), it is possible to directly visualize signaling crosstalk between receptors in cells. Prior work has specifically implicated Fyn for its ability to integrate information emanating from different cell-surface receptors ${ }^{92,93}$. For instance, Fyn is plays a crucial role in cell survival and myelination of neurons by oligodendrocytes, specifically by integrating integrin signaling with growth factor signaling. Oligodendrocyte precursors are able to survive and subsequently wrap neurons with a myelin sheath, even in low growth factor conditions through the additive effects of integrin and RTK signaling ${ }^{94-}$ ${ }^{98}$. This signal integration is mediated through Fyn activity ${ }^{94,98}$. It is striking and highly significant that our biosensor reveals evidence of such signal integration, in single cell imaging experiments in-vitro. This shows that compartmentalized signaling crosstalk between different receptor classes may be a key feature of signaling systems (Fig. 11).

Biosensor imaging also shows Fyn activity signals to be pulsatile. Recent work has shown that oscillatory signaling patterns are key hallmarks of several signaling modules, including regulation by transcription factors ${ }^{99,100}$ as well as MAP kinases $^{78,80,81,101,102}$. Generally, pulses arise due to the presence of negative feedback loops, which may also be accompanied by additional modulators. Interestingly, there is some evidence that switching temporal activity patterns can alter cellular fate; for 
instance, pulsatile ERK activity leads to one output while persistent activity leads to another. We observe robust and spontaneous activity pulses of Fyn in very distinct cell types even in serum-starved cells plated on FN (Fig. 8), and these pulses are further modulated by growth factor stimulation. This behavior strongly suggests the presence of rapidly activated, negative feedback loops that directly modulate Fyn activity.

Fyn activity pulses may be functionally significant in light of its demonstrated role in regulating cell migration, adhesion and actomyosin remodeling. In migration, there is an intrinsic periodicity in cycles of actin polymerization/remodelling/depolymerisation, periods of membrane remodelling as well adhesion assembly and disassembly. A protein that regulates several of these processes is conceptually much more likely to have pulses of activity versus sustained activity. In this light, its striking we are able to pick up 'activity pulses' at specific locations in the cell. This would need to be explored further in the context of directed migration and control of cell proliferation. Further, its indeed striking that membrane dynamics is reduced in the regions/zones where Fyn is more active (Fig. 10). Spatially-localized pulsatile activation may likely cause transient but limited stabilization of signaling clusters which would be difficult to achieve with sustained activation. These observations taken together suggest that Fyn activity may be correlated with a 'poised' membrane state and formation and stabilization of specific integrin-FAK dependent signaling clusters, which can be further tuned by growth factor signaling. Such specific control of adhesion and actomyosin dynamics may be important in Fyn's ability to mediate directed cell migration, growth control and tissue invasion. Context dependent and spatio-temporally regulated Fyn activity may provide a new perspective on understanding Fyn's roles in regulating diverse and sometimes opposing cellular functions. Fyn activity profiles also provide a direct readout of highly localized signaling crosstalk between distinct receptor classes. Overall, this work sets the stage for detailed investigations of such functional signaling crosstalk in numerous cellular contexts and offers a new paradigm for direct visualization of signaling dynamics mediated by Fyn and other Src kinases.

\section{Accession Numbers and Data Availability}

The coordinates of FYN-SH3/ Monobody-binder (F29) complex are deposited in the PDB under accession codes 5ZAU and associated NMR chemical shift data were deposited in BMRB under accession code 36164. MATLAB codes for image processing and computational analysis, other data and plasmid constructs used in the article are available from the corresponding authors upon reasonable request.

\section{Acknowledgements}

This research was supported by the Dept. of Biotechnology (DBT) (Grant \# BT/PR8071/MED/32/290/2013), Ministry of Science and Technology, Govt. of India and financial support from InStem and DBT, India. Additional support came from grant \# BT/IN/BMBF/03/AG/2015-16 and BT/IN/Denmark/07/RSM/2015-2016 from DBT, India. We thank Professor S. Ramaswamy and the Technology for the Advancement of Science (TAS) theme for support. A.M. was supported by The University Grants Commission (UGC), India with JRF-SRF fellowships. Financial support from the DBTRA Program to RS in Biotechnology and Life Sciences is gratefully acknowledged. We thank Dr. Sandeep Krishna, Debshankar Banerjee (NCBS) and Prof. Sugata Munshi (Jadavpur University) for helpful discussion regarding image analysis. We thank Prof. 
Klaus Hahn (University of North Carolina, Chapel Hill) and Professor Brian Kay (University of Illinois, Chicago) for various DNA constructs. We thank Dr. Colin Jamora (inStem) and Dr. Arati Ramesh (NCBS) for critical reading of the manuscript and helpful discussions. We thank Professor Satyajit Mayor (NCBS) and his group for discussions, sharing reagents and technical help. We thank members of the Gulyani lab and other colleagues from inStem and NCBS for discussions. We thank Nishan Shettigar for help with manuscript and discussions. We thank Central Imaging \& Flow Cytometry Facility of NCBS and inStem, especially Dr. H. Krishnamurthy, Dr. Manoj Mathew and Dr. Feroz M. H. Musthafa for support with flow-cytometry and microscopy experiments. We also thank the sequencing facility and lab support at NCBS-inStem.

\section{Author contributions}

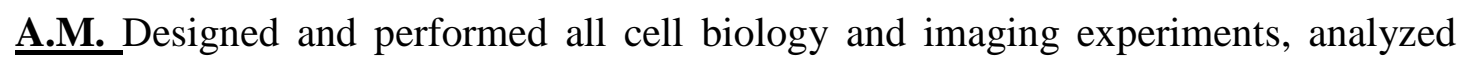
imaging and biosensor data, carried out biosensor optimization in cells, helped perform binder screening and co-wrote the manuscript. R.S. Designed experiments and engineered the genetically encoded biosensor and developed constructs used in the study, performed biochemical and immunoblotting assays, analyzed biochemical and biosensor data, helped perform binder screening and co-wrote the manuscript. $\underline{\text { S.U. }}$ Performed binder screening and analysis. S.B Performed image quantification and analysis using MATLAB scripts. P.P. Performed NMR acquisition and structural analysis. S.M. Performed binder screening and carried out biochemical assays. $\underline{\text { G. }}$ Performed biochemical assays. S.D.K. Wrote and implemented MATLAB code for image processing. R.D. Oversaw structural analysis, designed NMR experiments and carried out structural analysis and co-wrote the manuscript. B.M.R. Designed and oversaw yeast surface display screening, provided yeast-display library, co-wrote the manuscript. A.G. Conceived designed and oversaw the study, designed experiments, analyzed data and wrote the manuscript with input from other authors.

\section{Competing interests -}

The authors declare no competing interests 


\section{Methods}

\section{Reagents and Cell-culture maintenance}

Chemicals, antibodies, primer and DNA constructs information are provided in the supplementary section. Mycoplasma negative cell lines, Human Embryonic Kidney Cells (HEK-293T), Amphopack-293, Mouse Myoblast (C2C12) and Human Bone Osteosarcoma Epithelial Cells (U2OS) were used. HEK-293T, Amphopack-293, and C2C12 cells were cultured in DMEM media supplemented with $10 \%$ FBS with $1 \%$ Penicillin Streptomycin (Pen-Strep, $10^{3} \mathrm{U} / \mathrm{mL}$ ) solution. U2OS cells were grown in McCoy's 5A media supplemented with Sodium Carbonate, 10\% FBS and 1\% PenStrep.

Protein purification and Binder Screening-The details are described in supplementary section.

\section{Sub-cloning of SH3 domains and binder F29}

The sequence of thrombin cleavage site 'thr' $(L V P R G S H)$ of vector pet $14 \mathrm{~b}$ was changed to Prescission protease cleavage site 'ppx' (LEVLFQGP) using the quick change mutagenesis (Supplementary Fig. 6a) with SN 1, 2 primes to yield plasmid p-SUMOppx-Fyn SH3.Similarly, ppx site was introduced in other SH3 domains. plasmids pSUMO-ppx-Src SH3-T99D has been constructed using site directed mutagenesis with primers SN 4, 5. Binder F29 ORF was amplified (primers SN 6, 7) and product was digested with $\mathrm{NdeI}$ and $\mathrm{BamH1}$ and sub-cloned in p-SUMO-ppx-Fyn SH3 to yield plasmid p-SUMO-ppx-F29. Furthermore, F29-ORF was sub-cloned along with linker $(\text { GGGS })_{3}$ into pGX6P-1 vector under Bam H1 and XhoI site using overlap extension PCR with primers SN 8,9 yielding plasmid p-GST-ppx-L ${ }_{15}-\mathrm{F} 29$ (Supplementary Table 1, 2).

\section{Binder-Fyn SH3 domain complex structure determination}

(i) NMR Studies-Protein purification for NMR studies is described in supplementary section. All NMR spectra were acquired at $298 \mathrm{~K}$ on an $800 \mathrm{MHz}$ Bruker Avance III spectrometer (NMR facility-NCBS). Assignment of backbone resonances of the proteins were carried out using HNCACB and CBCACONH experiment. ${ }^{1} \mathrm{H}$ and ${ }^{13} \mathrm{C}$ resonance assignments of side chain atoms in F29 were obtained by collecting $\mathrm{H}(\mathrm{CC}) \mathrm{CONH}$ and $(\mathrm{H}) \mathrm{CC}(\mathrm{CO}) \mathrm{NH}$ spectra, respectively. A $3 \mathrm{D}^{15} \mathrm{~N}$-edited NOESY experiment was carried out on a complex of ${ }^{15} \mathrm{~N}$, ${ }^{2} \mathrm{H}$-labeled Fyn-SH3 and unlabeled F29. All the experimental data were processed using NMR pipe ${ }^{103}$ and TOPSPIN3.2 software. Analysis of NMR data was carried out by using Sparky ${ }^{104}$. Backbone assignments were obtained by PINE-NMR ${ }^{105}$ and confirmed manually. Structural model of F29 was calculated by CS-ROSETTA ${ }^{106}$. NMR titration was performed by titrating unlabeled F29 (ligand) to a sample of ${ }^{15} \mathrm{~N}$-labelled Fyn-SH3 domain (protein). At least 6 different protein: ligand ratios were collected ranging from 1:0.5 to $1: 4 .{ }^{1} \mathrm{H}_{-}{ }^{15} \mathrm{~N}$ HSQC spectra were taken at each titration point. The reverse titration was done by adding unlabeled Fyn-SH3 to labeled F29. 
(ii) Structure calculation-The structure calculation of the Fyn-SH3/F29 complex was performed using HADDOCK ${ }^{55}$. The input structure of Fyn-SH3 is from pdb: 3UA6, and that of F29 is the output lowest energy structure from CS-ROSETTA. The ambiguous restraints were obtained from the CSPs observed in the NMR titration data. Unambiguous restraints obtained from the NOESY spectra were included in the structure calculation. All restraints were used during the docking steps. The interface of F29 and Fyn-SH3 were kept semi-flexible during simulated annealing and the water refinement.

\section{Biosensor construction}

We have constructed intermolecular FRET-donor \& acceptor biosensor using h-Fyn gene and binder (F29), respectively. In the FRET-donor, mCerulean ( $\lambda_{\mathrm{ex}}=435 \mathrm{~nm}$, $\left.\lambda_{\mathrm{em}}=475 \mathrm{~nm}\right)$ and in FRET-acceptor, mVenus $\left(\lambda_{\mathrm{ex}}=515 \mathrm{~nm}, \lambda_{\mathrm{em}}=525 \mathrm{~nm}\right)$ were used. The source of mCerulean and mVenus were from pTriEx-mCerulean-Rac1 WT and pTriEx-mVenus-CBD constructs, respectively (gifted from Prof. Klaus Hahn's Lab University of North Carolina Chapel Hill). The h-Fyn gene source was from pRK5-cFyn (a gift from Dr. Filippo Giancotti, Addgene plasmid \# 16032). Biosensors were constructed using gene fusion, quick change mutagenesis, and overlap extension PCR methods ${ }^{107-109}$. The key steps are shown in Supplementary Fig. 6. Biosensors were either made in their original plasmid or in form of PCR fusion-product and were subcloned in the pTriEx-4neo vector (Novagen) under NcoI and BamH1 site. The strategies used during biosensors construction are summarized here in a step wise manner.

(i) FRET-donor- Flexible linker of (GGGGS) ${ }_{3}$ and its coding nucleotide sequence (5'GGTGGAGGCGGTTCAGGCGGAGGTGGCTCTGGCGGTGGCGGATCG-3') was incorporated between 243-244 nucleotide of h-Fyn ORF of pRK5c-FynWT plasmid using primer SN 14, 15 with quick change mutagenesis method to yield plasmid pRK-UD-L 15 -FynWT. In the next step mCerulean ORF was amplified from pTriEx-mCerulean-Rac1WT plasmid using primer SN 16, 17. Gel eluted product was used as a mega-primer in the overlap extension PCR using pRK-UD-L $15-$

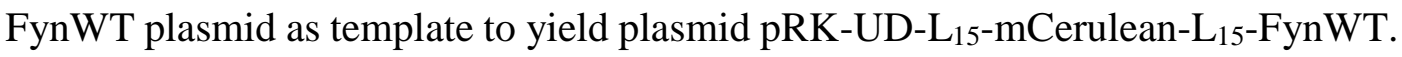
The ORF of this modified kinase was mutated (Y527F, constitutively active open kinase) with primer SN 18, 19 using site directed mutagenesis (SDM) to yield plasmid pRK-UD-L $\mathrm{L}_{15}-\mathrm{mCerulean}-\mathrm{L}_{15}$-FynCA. Further, these newly modified WT and CA ORFs were amplified using primer SN 20, 21. The product was digested with $N c o$ I and BamH1 and sub-cloned into pTriEx-4neo vector yielding plasmid pUD-L 15 -mCerulean-L 15 -FynWT/CA. Several plasmids were also constructed for control studies. The ORF of unmodified Fyn kinase WT was amplified with primer SN 20, 21 from pRK5c-FynWT plasmid. The amplified product was sub-cloned in pTriEx-4neo vector under NcoI and Bam $\mathrm{H} 1$ site yielding plasmid p-FynWT. Using SDM we have made p-Fyn CA. mCerulean ORF was cloned at the 3' end of Fyn WT ORF flanked by flexible linker yielding plasmid p-UD-FynWT-L 15 -mCerulean.

(ii) FRET-acceptor-mVenus \& F29 ORF was amplified using primer SN 17, 22 and 24,25 , respectively. These amplified products have an overlap in the coding sequence of linker, (incorporated through PCR). Using these products as a template 
and primer SN 22, 25, overlap PCR was performed. The fusion product mVenus$\mathrm{L}_{15}$-F29 was further sub-cloned to pTriEx-4neo vector under NcoI and BamH1 site to yield plasmid p-mVenus-L ${ }_{15}-\mathrm{F} 29$. In this plasmid the myristoyl group (MGSSKSKPKDPS) was incorporated with primer SN 26, 27 using quick change mutagenesis yielding plasmid p-myr-mVenus- $\mathrm{L}_{15}-\mathrm{F} 29$. We have also made the point mutant P41A in the F29 ORF of these acceptor biosensors with primer SN 10, 11 yielding plasmids p-mVenus-L ${ }_{15}-\mathrm{F} 29 \mathrm{P} 41 \mathrm{~A}$ and $\mathrm{p}-m y r-\mathrm{mVenus}_{15}-\mathrm{L} 29 \mathrm{P} 41 \mathrm{~A}$ respectively. The mCerulean, $\mathrm{mVenus}$ ORFs were amplified with primer SN 22, 23 from original source plasmids and further sub-cloned in pTriEx-4neo vector under NcoI and BamH1 to yield p-mCerulean, p-mVenus, respectively.

In-vivo biosensor characterization- The details methods are described in supplementary section.

\section{Cell treatment and imaging conditions}

For single-cell imaging experiments, transient transfections in all the cells (U2OS, C2C12 and Fyn-KD-HEK 293T) were carried out using Lipofectamine 3000 according to the manufacturer's instruction (Life Technologies). Cells were checked for expression of plasmid of interest after 18 hours post transfection by fluorescence microscopy. Cells were then starved for 4-6 hours prior to being plated on glassbottomed dishes coated with desired density of fibronectin (FN) (see Supplementary information) and allowed to adhere for 30-40 minutes prior to imaging.

Serum-starved cells plated on FN were imaged for 25 minutes (acquisition settings are mentioned in following section) to record basal Fyn activity (activity in response to integrin activation/engagement) in a serum-starved state. For stimulation experiments, cells were initially imaged for 5-6 minutes to record basal Fyn activity. PDGF at a final concentration of $10 \mathrm{ng} / \mathrm{ml}(20 \mu \mathrm{g} / \mathrm{ml}$ stock in 1X DPBS) was then added onto the cells and images for an additional 15-16 minutes were acquired.

For treatment of cells with FAK inhibitor (PF-562271), serum-starved cells plated on FN were imaged for an initial period of $10 \mathrm{~min}$ and the inhibitor PF-562271 was added at a final concentration of $10 \mu \mathrm{M}(10 \mathrm{mM}$ stock in $100 \%$ DMSO). Post inhibitor addition cells were imaged for an additional 10 minutes. The cells were then treated with PDGF (10ng/ml) and imaged for another 10 mins.

\section{Image acquisition}

All imaging experiments (unless mentioned) were carried on the Olympus FV3000 microscope equipped with an PLANAPO N 60X oil immersion objective with a NA = 1.42 attached to a stage-top live-cell incubator to maintain cells at $37^{\circ} \mathrm{C}$ with $5 \% \mathrm{CO}_{2}$ (Tokai Hit). The microscope has two High Sensitivity Detectors (HSDs). The microscope also has a Z-Drift compensator and a motorized XY-stage which allows for multi-point-time-lapse imaging. Dex-Dem images were acquired using an excitation wavelength of $405 \mathrm{~nm}$ and emission was collected on HSD1 and the bandwidth of 
collection was from (437-499) nm. Aex-Aem images were acquired using an excitation wavelength of $514 \mathrm{~nm}$ and emission was collected on HSD2 and the bandwidth of collection was (528-628) $\mathrm{nm}$. Dex-Aem images were acquired using donor excitation and emission was same as acceptor emission. Voltage and gain settings were kept same while imaging the same cell prior to and after any kind of treatment (Bleaching/addition of PDGF/addition of inhibitor)

(i) Acceptor Photo-bleaching Method-For cells expressing FynSensor or non-binding mutant, prebleach and post-bleach Dex-Dem, and Aex-Aem images were acquired as described above. Acceptor bleaching was performed using $514 \mathrm{~nm}$ laser at $2 \mathrm{X}, 5 \mathrm{X}$ and $10 \mathrm{X}$ light dosage for 10 seconds each $(\mathrm{X}=0.069 \mathrm{~mW}$ ) (refer to Fig. $5 \mathbf{b}$ \& c) and DexDem, and Aex-Aem images acquired. For determining the amount and pattern of the increase in donor fluorescence post acceptor photo-bleaching, a customized MATLAB program was used. Dex-Dem (donor channel) images were used for this analysis. All the images were subjected to background subtraction and a thresholding operation before analysis. A user-defined global threshold was first used to generate a binary mask using the donor channel pre-bleach image of each cell. This was then used to determine the boundary of the cell using Canny's edge detection algorithm ${ }^{110,111}$. A centroid of each cellular mask was also determined. A "linescan" algorithm was used to quantify the variation of donor fluorescence recovery with distance from the cell periphery. For this, lines from every point on the periphery of the cell to the centroid were generated. Donor fluorescent intensities (from raw images) along these lines were calculated, and averages of all intensities were obtained as a function of distance from the periphery. For a given cell, the donor intensity values before and after bleaching (Pre-bleach Donor $_{\text {and Post-bleach }}$ Donor) were thus determined and used in the following equation to calculate percentage recovery of donor fluorescence as per established methods ${ }^{112}$.

Donor recovery $(\%)=\left(1-\frac{\text { IPre-bleachDonor }}{\text { IPost-bleachDonor }}\right) * 100 \ldots \ldots \ldots \ldots \ldots \ldots$ Equation 1

Where,

I Pre-bleach ${ }_{\text {Donor }}$ - donor fluorescence intensity prior to bleaching.

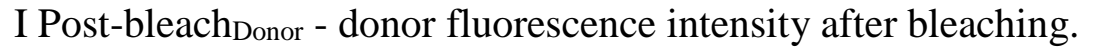

For generating the representative $\Delta$ Donor images, in Fig. 5a the prebleach donor channel image was subtracted from the postbleach donor channel image of the same cell $\left(\Delta\right.$ Donor $=$ Post-bleach ${ }_{\text {Donor-Pre-bleach }}$ Donor $)$ using MATLAB.

(ii) Donor Sensitized emission method-The Donor Sensitized Emission (SE) method records the FRET signal by measuring the amount of acceptor emission after excitation of the donor. The method used here has been modified from the original method as described earlier ${ }^{71}$ and requires the Dex-Dem, Dex-Aem and Aex-Aem images for calculation of FRET. To calculate the levels of both donor and acceptor bleed-through and direct acceptor cross-excitation using donor excitation, "donor only" and "acceptor only" samples were imaged in the following way:

i. Donor only sample: Dex-Dem and Dex-Aem

ii. Acceptor only sample: Dex-Aem and Aex-Aem 


\section{Image analysis}

All the image analysis was done using MATLAB and plots have been generated using Graph Pad Prism 5, unless otherwise specified. The customized codes written for the purpose of image analysis are available on request.

\section{Generation of FRET index image}

All images acquired were converted to 8-bit format prior to processing. FRET measurements were done on cells expressing both donor and acceptor fluorophores of FynSensor using equation 2, followed by a median filtering with a $2 \times 2$ pixel block around each pixel ${ }^{113}$. The Fiji plugin "FRET analyzer"114 was used to determine the bleed through values used in equation 2 from donor-only and acceptor-only cells.

FRET index images are shown using ImageJ (NIH) Fire LUT and scaled by an arbitrary number only for visual representations.

Briefly, for our sensitized FRET experiments, the following equation was used to generate the FRET index images:

FRET index image $=I_{\text {FRET }}-\left(\alpha_{D} I_{\text {Donor }}\right)-\left(\alpha_{A} I_{\text {Acceptor }}\right) \ldots \ldots \ldots$ Equation 2

Wherein,

$\boldsymbol{\alpha}_{D}-$ is the mean of the slope of the bleed-through signal of the donor alone (here 0.55), $\boldsymbol{\alpha}_{\mathrm{A}}$ - is the mean of the slope of the bleed-through signal of the acceptor alone (here 0.028 ),

IFRET- is the FRET channel image,

IDonor - is the Donor channel image, and,

$\mathbf{I}_{\text {Acceptor }- \text { is the Acceptor channel images. }}$

FRET index images depict total FRET $\left(\right.$ FRET $\left._{\mathrm{T}}\right)$

Calculations used are consistent with other literature reports on intermolecular FRET measurements, like the Fiji plugin "FRET analyzer"114 and another very recent report $^{115}$.

\section{Quadrant analysis}

For cellular area estimation, acceptor channel image time series (Aex-Aem) for each cell was processed, (global thresholding, smoothening by erosion operation and filling holes using imfill and removing small area particles by bwareafilt). Cellular area processed images (binary images with a value of 1 inside cell and 0 outside) hence generated, were used as masks to further process the FRET index images (or 'YFP channel' or 'CFP channel images as the case may be (Supplementary Fig. 18a, 19d). Cell centroids were determined on the binary masks using regionprops function. With respect to the centroid, a vertical and horizontal line was drawn to divide each cell into four quadrants as shown in Fig. 6a i. Mean fluorescent intensity (either FRET or 'CFP' or 'YFP') in each quadrant was calculated using Equation 3; wherein sum of the fluorescent intensity values from all pixels in a given quadrant is divided by total number of pixels in the quadrant. The mean intensity is followed over time and the variation is plotted in Fig. 8 a ii, b ii, $\mathbf{c}$ ii 


$$
\text { Mean intensity }=\frac{\sum_{i=1}^{n} \text { Pixel Value }_{i}}{n} \ldots \ldots \ldots \ldots \ldots \text { Equation } 3
$$

$n=$ no. of pixels in the specific quadrant

Quantifying spatio-temporal Fyn activity patterns

For testing if Fyn activity is spatially enhanced, mean FRET $_{\mathrm{T}}$ values (see above) for every quadrant in each cell was analyzed over time. Max-FRET ${ }_{\mathrm{T}}-\mathrm{HFQ}$ (HFQ: highFRET quadrant) refers to the maximum value of mean quadrant FRETT seen in the time series, and was determined for each cell. Max-FRET ${ }_{\mathrm{T}}$-LFQ (LFQ:low FRET quadrant') was the corresponding mean $\mathrm{FRET}_{\mathrm{T}}$ value for lowest FRET quadrant at that specific time point, for the given cell. Max-FRET ${ }_{\mathrm{T}}$-HFQ and Max-FRET $\mathrm{T}_{\mathrm{T}} \mathrm{LFQ}$ were averaged over multiple cells for both serum-starved as well as PDGF-stimulated cells.

For calculating PDGF-induced enhancement in Fyn activity, difference in the FRET intensity levels (Max-FRET T-HFQ) before and after PDGF stimulation were calculated. Also calculated were differences in Max-FRET ${ }_{\mathrm{T}}-\mathrm{LFQ}$ before and after PDGF.

\section{Characteristic Time Period of FRET pulses}

Power spectral density (PSD) ${ }^{116}$ of the time series was computed using periodogram function. Prior to subjecting the $\mathrm{FRET}_{\mathrm{T}}$ time traces to frequency decomposition analysis, we removed any quadratic trend using the detrend function in line with established procedures ${ }^{117,118}$. The frequency against which maximum power ${ }^{116,119}$ is obtained, known as dominant frequency in the time series, was used to calculate the time-period of $\mathrm{FRET}_{\mathrm{T}}$ intensity oscillations in each quadrant. The frequency is converted to time period based on the sampling frequency, and is plotted in the Fig $\mathbf{8 d}$.

\section{Quantifying active Fyn localization at membrane.}

The maximum FRET intensity region of cell membrane was identified through line scanning.

An arc of arbitrary length unit (40 pixels here) on the membrane encompassing this region of maximum FRET intensity was selected, and a sector was constructed which spanned the membrane at the cell edge and extended towards the centroid of the cell. The intensity in this sector was measured over time and plotted as a function of distance from the cell edge (Fig. 8e)

\section{Cell morpho-dynamics analysis}

Donor channel images (Dex-Dem) were used to generate a cell mask and calculate the cellular area at each time point (see above). Two consecutive cell mask images were used to determine the temporal change in the cellular area $\left(\Delta A_{i}=A_{i+1}-A_{i}\right)$. The absolute value of this temporal change in cell area was then normalized for the initial cell area $\left(\left|\Delta \mathrm{A}_{\mathrm{i}}\right| / \mathrm{A}_{1}\right)$ to account for differently sized cells. This was then used to calculate the mean fractional area change to reflect the cumulative area changes over a period of time 
(Equation 4a). Similarly, cumulative cell perimeter changes were determined using the modulus of change in perimeter between consecutive cell frames, normalized for the initial cell boundary length (Equation $\mathbf{4 b}$ ).

$$
\begin{aligned}
& \text { Mean Fractional Area Change }=\frac{\sum_{1}^{n-1} \frac{\left|\Delta \mathrm{A}_{\mathrm{i}}\right|}{\mathrm{A}_{1}}}{n-1} \ldots \ldots \ldots \ldots \ldots \ldots \ldots \ldots \ldots \ldots \ldots \text { Equation } 4 \mathbf{a} \\
& \text { Mean Fractional Perimeter Change }=\frac{\sum_{1}^{n-1} \frac{\left|\Delta \mathrm{P}_{\mathrm{i}}\right|}{\mathrm{P}_{1}}}{n-1} \ldots \ldots \ldots \ldots \ldots \ldots \ldots \text { Equation } 4 \mathbf{b}
\end{aligned}
$$

In the above equation, $\mathrm{n}$ is total numbers of frames recorded. Once mean fractional area and perimeter changes were determined for a single cell, averages from multiple such cells were then calculated for each of the parameters in each set has been plotted Supplementary Fig. 12. Also see Fig. 10a ii, iii.

\section{FRET intensity and membrane motility correlation}

Intracellular quadrant area changes have been described through a parameter (temporal quadrant area change; $\mathrm{T} \Delta \mathrm{QA}$ ), wherein the temporal change (between time $\mathrm{i}$ and $\mathrm{i}+1$ ) in quadrant area has been normalized to the area of the quadrant at time $\mathrm{i}$. This was done to rationalize for relative area changes in each quadrant.

$$
\mathrm{T} \Delta \mathrm{QA}=\frac{\left(\left|\mathrm{QA} A_{\mathrm{i}+1}-\mathrm{QA} \mathrm{i}\right|\right)}{\mathrm{QA}_{\mathrm{i}}} \ldots \ldots \ldots \ldots \ldots \ldots \ldots \ldots \text { Equation } 5
$$

Where, $\mathrm{QA}_{\mathrm{i}}$ is the quadrant area at the $\mathrm{i}^{\text {th }}$ time point.

$\mathrm{T} \Delta \mathrm{QA}$ was determined for each cellular quadrant, for each time point. This temporal change in cell area was then mapped to the mean FRET $_{\mathrm{T}}$ intensity of the $\mathrm{i}^{\text {th }}$ frame, for each time point and each individual quadrant and is plotted in the graph Fig. 10 b ii. For the purpose of FRET-area change mapping, the FRET $_{\mathrm{T}}$ intensity data was binned into lengths of 2 intensity units $[(10,12),(12,14)]$ and so on. For each $\mathrm{FRET}_{\mathrm{T}}$ bin, the mean and the SEM of the linked quadrant morphology (T $\triangle \mathrm{QA})$ values were determined and plotted. This was done to investigate the correlation between quadrant area changes and FRET $_{\mathrm{T}}$ levels. The curve-fitting was done using the Polynomial Quadratic equation $\left(\mathbf{f}=\mathbf{y}_{0}+\mathbf{a}^{*} \mathbf{x}+\mathbf{b}^{*} \mathbf{x}^{2}\right)$ on SigmaPlot (see Fig. 10 b ii). 

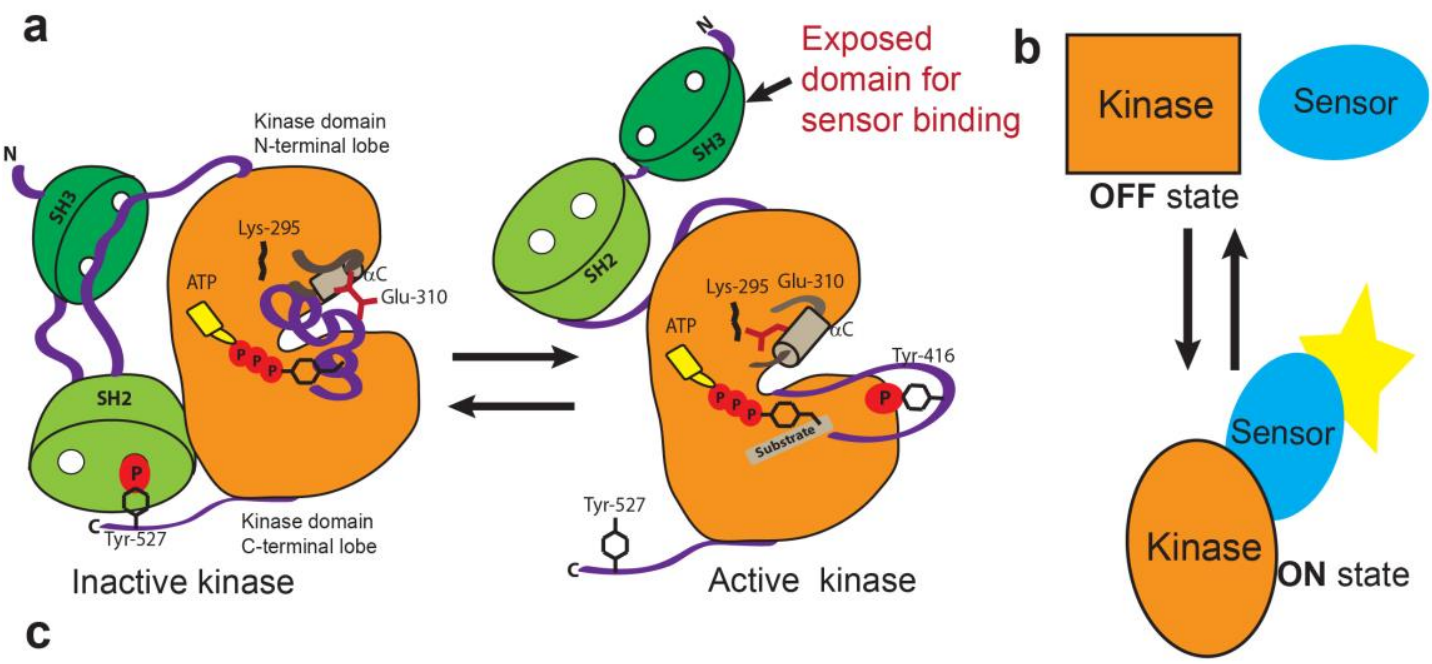

C

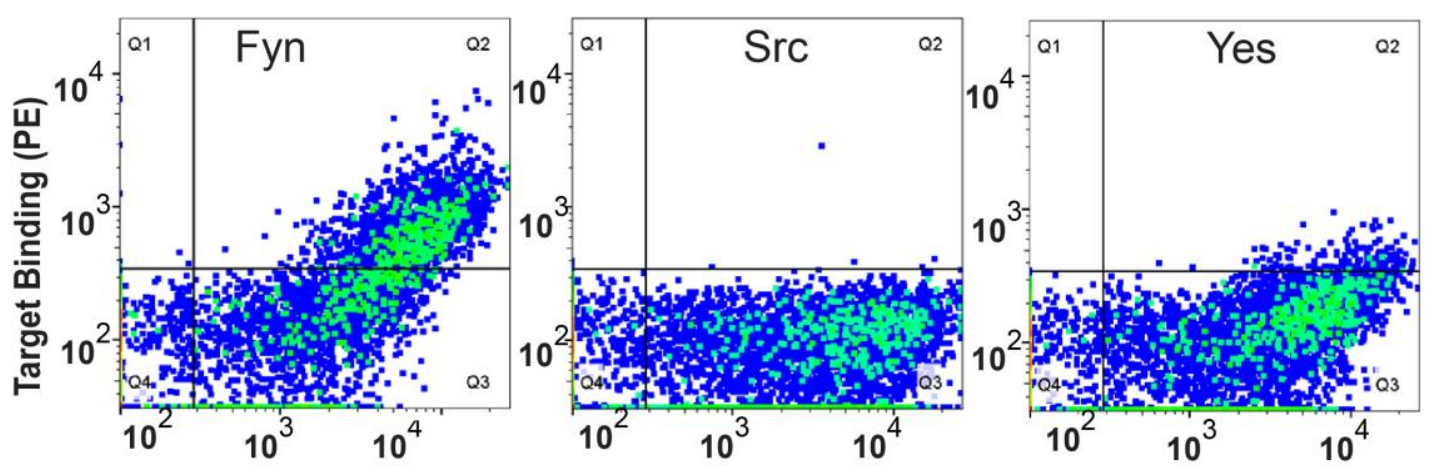

Library scaffold expression (Alexa fluor-633)

d

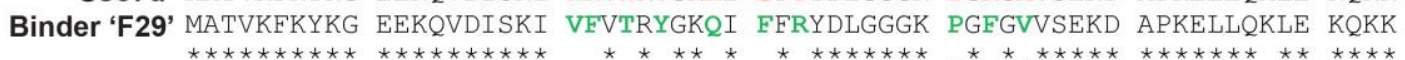
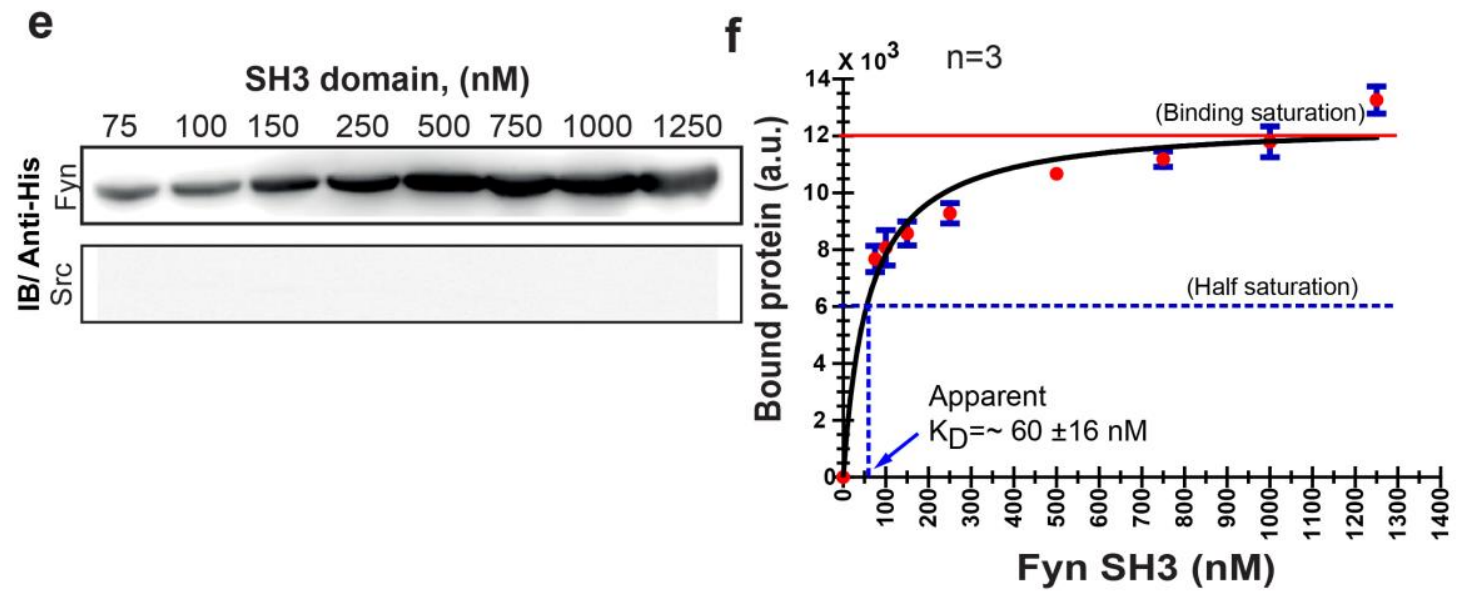

Fig. 1: High-throughput screening yields a new protein binder for generating a specific biosensor for active Fyn.

(a) Rationale for a biosensor targeting active conformation of SFK, Fyn. A cartoon depicts how activation of Fyn kinase leads to a more open conformation and buried regions (including its $\mathrm{SH} 3$ domain) becoming more accessible for biosensor binding. Inactive (left) SFKs are held in a closed conformation through two key intramolecular interactions (for instance see Cell, 2001, 105, 115-12) that are lost in the open state 
(right). (b) Schematic diagram representing sensing of active and open state of kinase by an active state sensor. (c) High-throughput combinatorial library screening yields a protein binder specific for Fyn SH3 domain. Analysis of the yeast clone 'F29' using fluorescence activated cell sorting (FACS) shows F29 preferentially binds Fyn while showing little or no binding to SH3 domains of ubiquitously expressed SFKs, c-Src and Yes $(1 \mu \mathrm{M}$ each). Here, fluorescence signal due to biotin-labeled target SH3 (Streptavidin-PE) is plotted against signal due to yeast cells expressing the c-myctagged Sso7d scaffold (Alexa 633 labelled anti-c-myc). Detection of fluorescence signal due to Fyn-SH3 along with anti-c-myc fluorescence in a single yeast cell, indicates expression of full-length F29 fusion-protein and binding to labelled Fyn-SH3 target. (also see Supplementary Fig. 2) (d) Comparison of amino acid sequences of new binder F29 with original Sso7d scaffold. 10 Sso7d residues randomized for library generation are shown marked in red while corresponding new residues in binder F29 are shown in green. (e) Binder F29 is able to recognize Fyn SH3 outside the context of yeast surface with recombinantly expressed and purified F29 showing saturable binding to Fyn SH3. Immunoblot showing pull-down of His-tagged Fyn or Src SH3 domains (75 $\mathrm{nM}$ to $1.25 \mu \mathrm{M}$ ) using GST-F29 fusion protein immobilized as bait on glutathione sepharose beads. His-tagged SH3 protein detected via anti-6X-His antibody (also see Supplementary Fig. 3). (f) Amount of bound Fyn SH3 plotted against total input Fyn $\mathrm{SH} 3$. The data from three independent binding experiments $(\mathrm{n}=3)$ was plotted and fitted assuming 1:1 binding using a GraphPad Prism module. The apparent $\mathrm{K}_{\mathrm{D}}$ values were calculated using Supplementary Equation 1 (refer to Supplementary methods). Values are mean \pm s.e.m. 

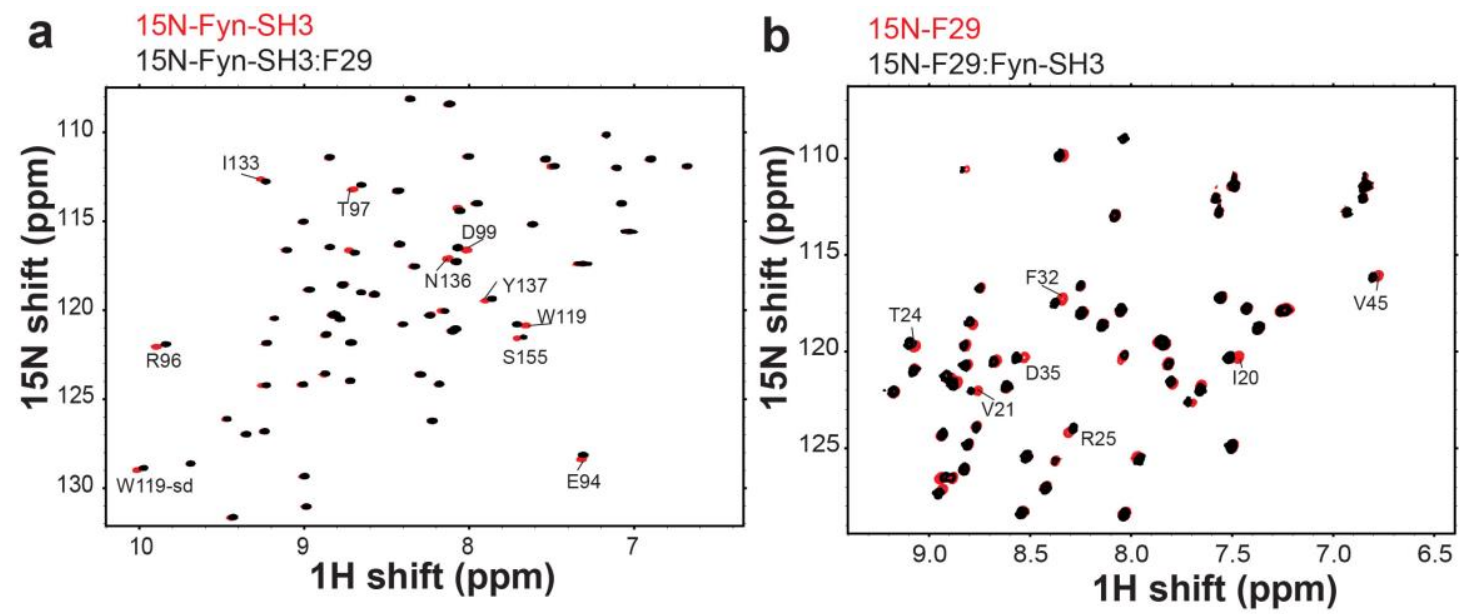

C

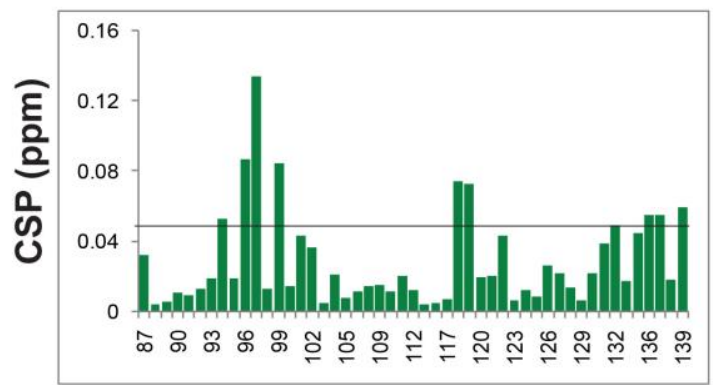

Fyn-SH3 residues

d

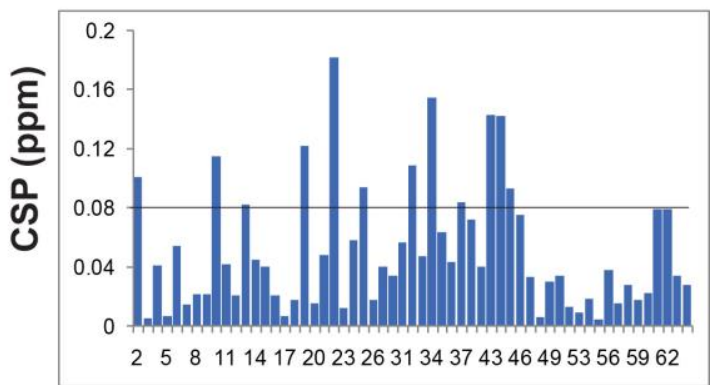

e

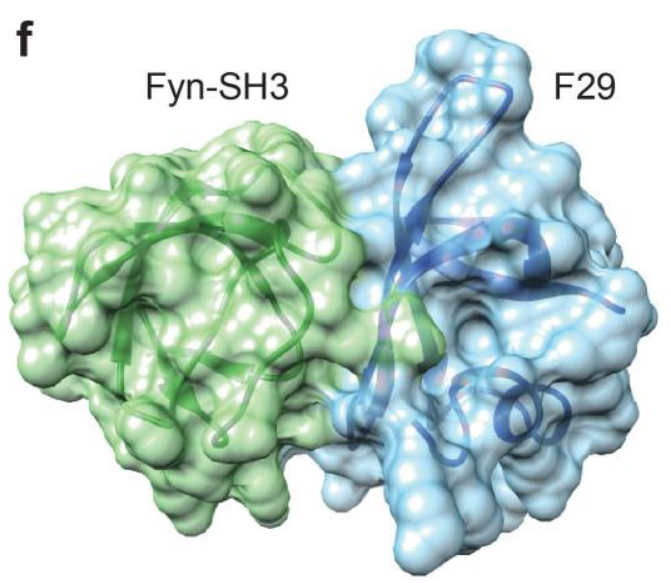

Fig. 2: NMR analysis yields structure of the Fyn-SH3 complex with newly isolated binder.

(a) ${ }^{15} \mathrm{~N}$-edited heteronuclear single quantum coherence (HSQC) spectrum of Fyn $\mathrm{SH} 3$ (red) overlapped with the spectrum of F29-bound Fyn-SH3 (black). (b) ${ }^{15} \mathrm{~N}$-edited HSQC spectrum of F29 (red) overlapped with the spectrum of Fyn-bound F29 (black). The peaks and assigned residues with significant chemicals shifts are marked in both $\mathbf{a}$ and $\mathbf{b}$. Chemical shift perturbations (CSP) observed in $\mathbf{a}$ and $\mathbf{b}$ are plotted in (c) and (d), respectively. A horizontal line is drawn at $2 \sigma$, where $\sigma$ is the standard deviation of the CSP values. (e) The lowest energy structure from Haddock is provided, where FynSH3 is colored green and F29 is colored in light blue. (f) The surface representation of e, where the surface of Fyn-SH3 and F29 are colored in green and light blue, respectively. 
a

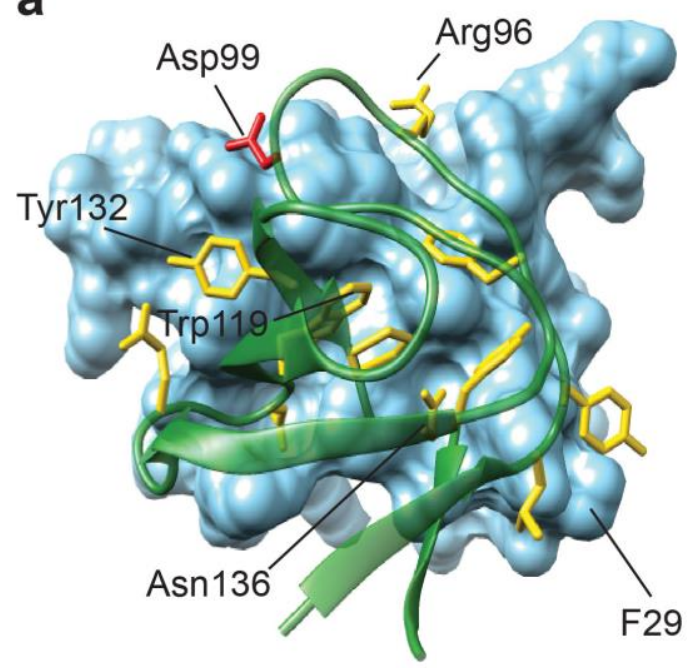

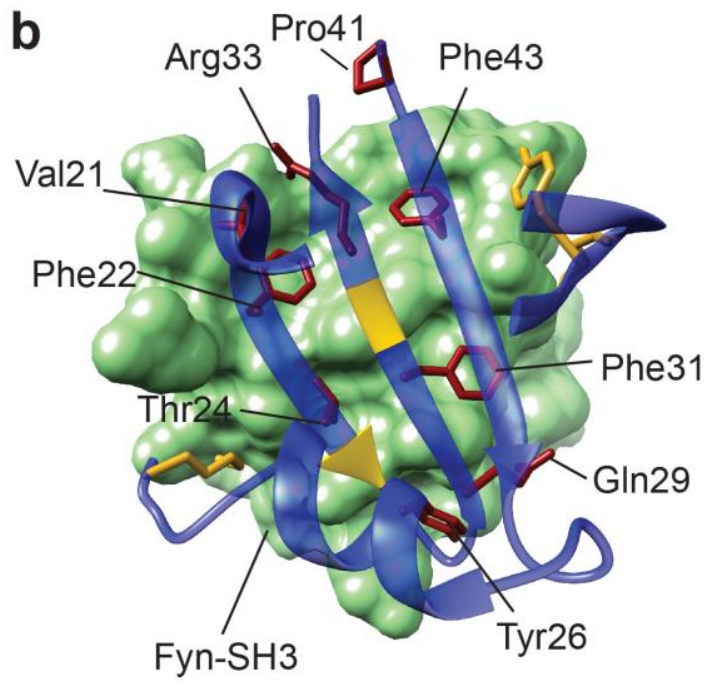

C

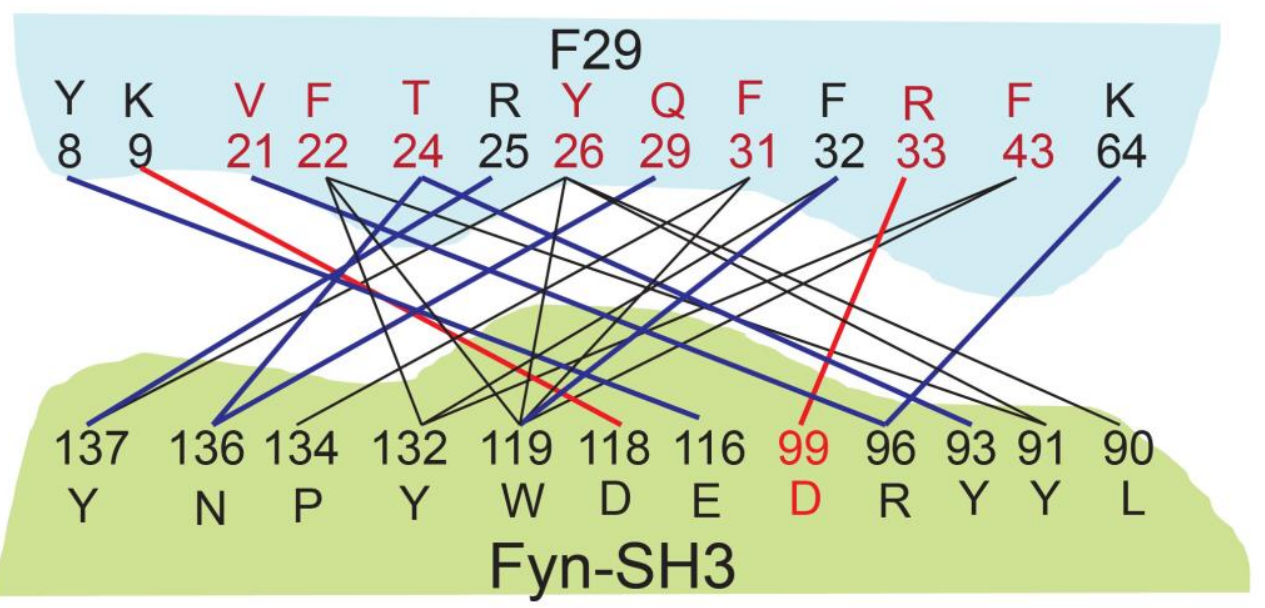

d
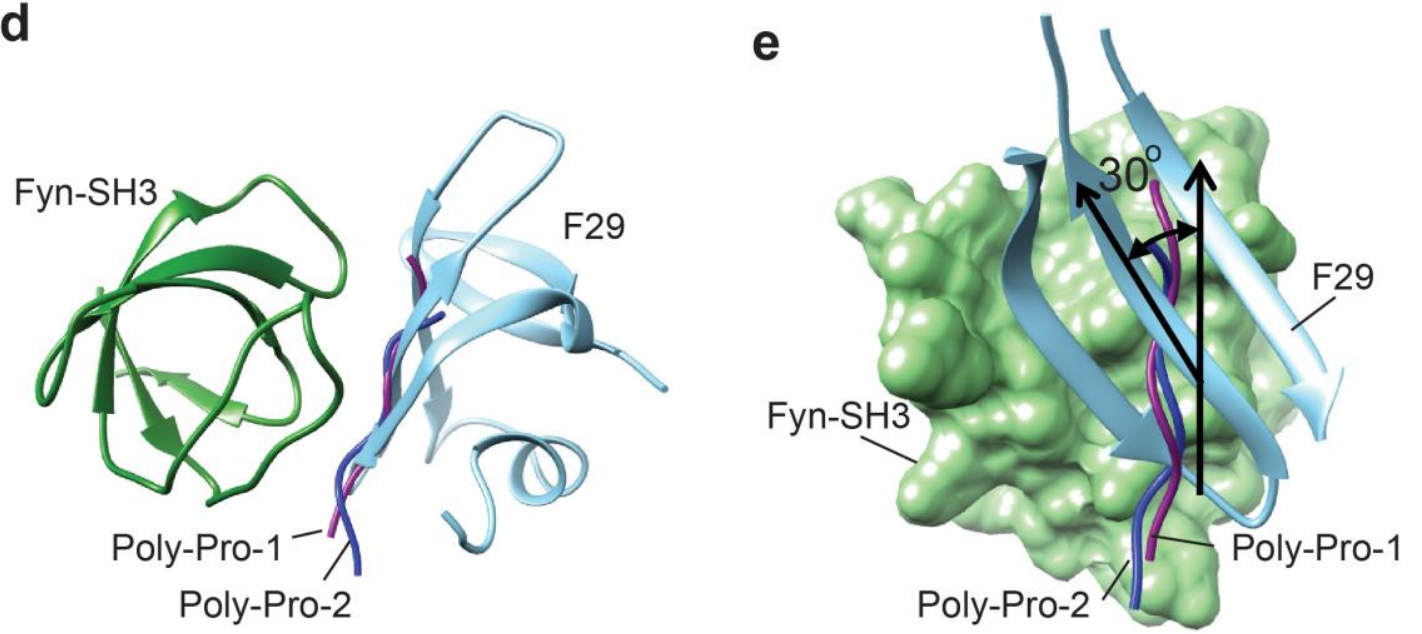

Fig. 3: Binding interface of the Fyn-SH3:F29 complex.

(a) Ribbon diagram of contact interface: A view of the contact interface where the F29 surface is represented in light blue and Fyn-SH3 is shown in green ribbon. The interacting side chains of Fyn-SH3 are shown in yellow and the key interacting residue Asp99 (see below) side chain is shown in red. (b) Complementary view of interface: Complementary view of the interface with Fyn-SH3 in green and F29 in blue (ribbon diagram) is shown. The interacting side chains of F29 residues are shown and identified 
here. They are colored in dark red if randomized and colored in gold otherwise. The non-randomized residues Arg25 and Phe32 interact with Fyn-SH3 through the backbone atoms and hence, their ribbons are colored in gold. Non-interacting regions of F29 far away from the interface are hidden for clarity. (c) Schematic showing FynSH3:F29amino acids involved in interfacial contacts. The hydrophobic and aromaticaromatic contacts between specific residues are shown in black lines, the hydrogen bonds are shown in blue lines and salt-bridges are shown in red lines. Residues are colored in red if randomized and colored in black otherwise. Asp99 in Fyn-SH3 is colored in red. (d) Comparison with SH3-polyproline binding: An alignment of the Fyn-SH3:F29 complex with two structures of Fyn-SH3:poly-proline complexes (pdb:4eik and pdb:3ua7). (e) A rotation of $\boldsymbol{d}$ to show a comparison between the two interfaces. F29 is differently aligned as compared to the poly-prolines when bound to $\mathrm{SH} 3$ domain. The angle between poly-prolines and the $\beta$-sheet of F29 is $\sim 30^{\circ}$ with respect to the $\mathrm{SH} 3$ domain. 
a (i) Exogenously expressed Fyn Kinase pull down

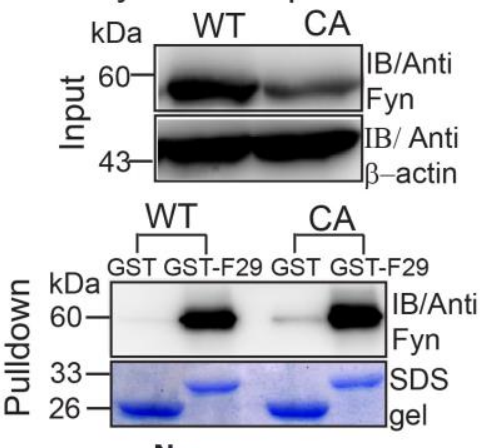

(ii)

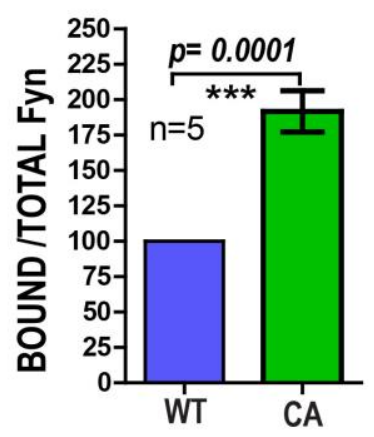

b Endogenous Fyn kinase pull down
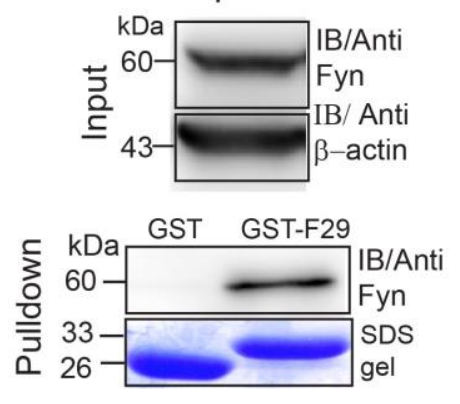

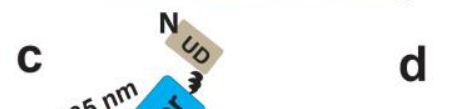

d

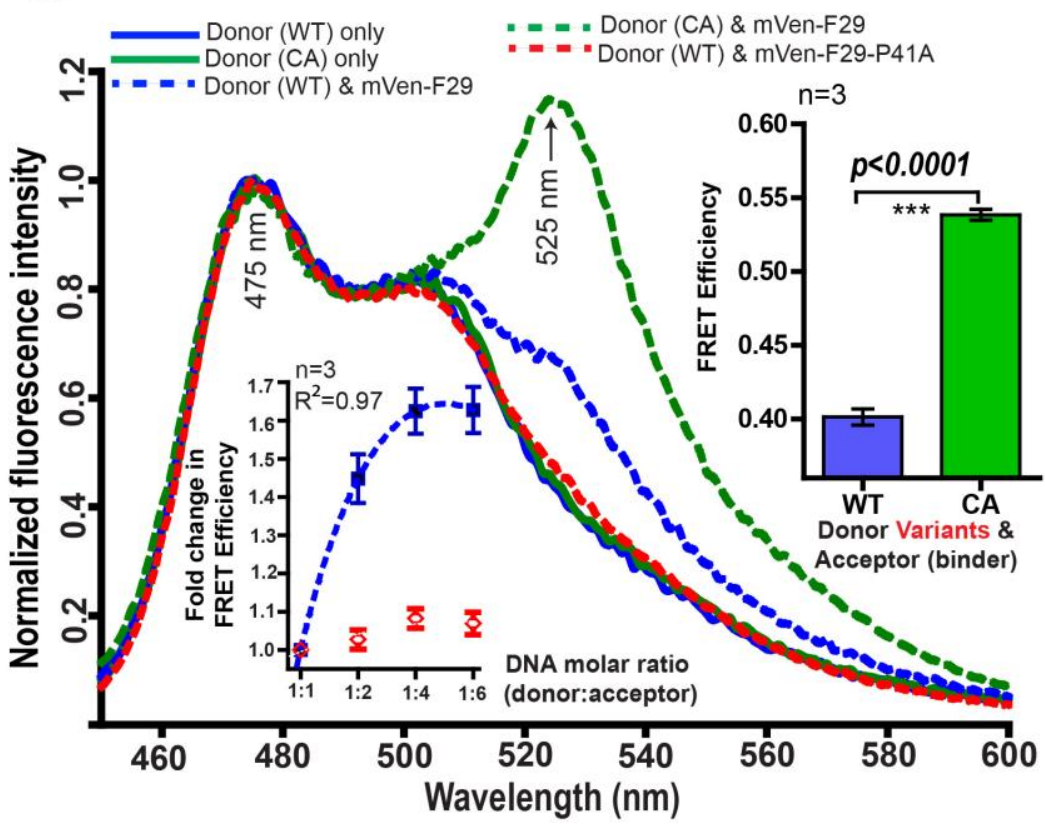

e (i)

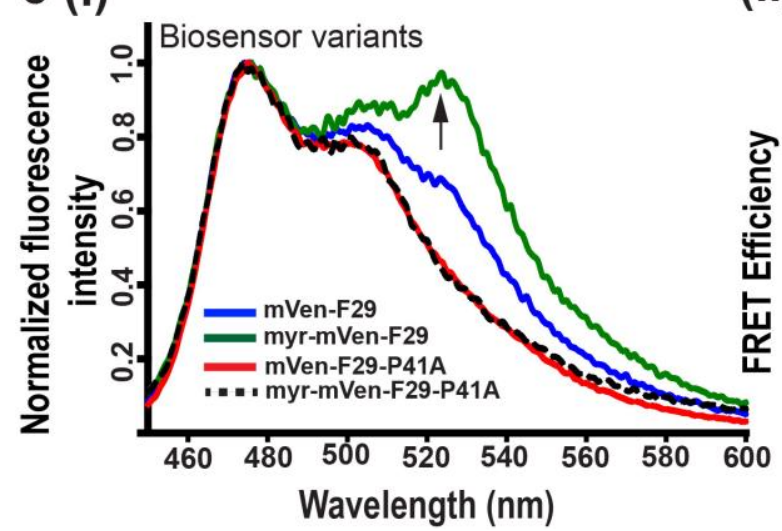

(ii)

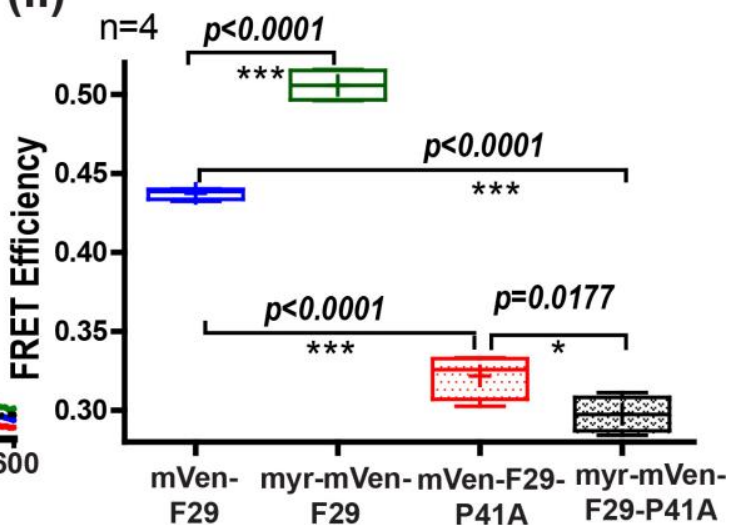

Fig. 4: Developing a FRET biosensor for sensing active Fyn in cells.

(a) F29 preferentially binds active Fyn kinase. (i) Immunoblots showing GST-F29 pulldowns of cellular Fyn after transfecting cells with either Fyn WT (wild type) or Fyn CA (constitutively active kinase). (ii) Extent of pulldown with either CA or WT Fyn. The band intensity of pulled-down Fyn kinase from five such experiments was quantified and normalized to expression level. Binder F29 is able to pull-down CA 
mutant of Fyn to a greater extent than WT. Values are mean \pm s.d from least five independent experiments. Data was compared using Student's unpaired one-tailed ttest $(* * * p=0.0001)$ on GraphPad Prism. (b) F29 binds full-length, endogenous cellular Fyn kinase. Immunoblots, using anti-Fyn antibody, show pulldown of full-length endogenous Fyn from cell lysates by GST-F29 immobilized on glutathione beads $(n=3)$. GST coated beads are shown as controls. Coomassie stained gels of the bait proteins, either GST-F29 or GST used for pulldown are also shown. (c) Schematic diagram and model for an intermolecular FRET biosensor for visualizing active Fyn in cells. The donor fluorophore (mCerulean) is inserted in between Fyn kinase unique (UD) and SH3 domains using flexible linker sequences to minimally disrupt kinase function (also see Supplementary Fig. 7, 10). Newly developed Fyn active-state binder F29 is fused with acceptor fluorophore (mVenus). In the cell, F29 binder (FRET-acceptor) would bind the exposed SH3 domain of active Fyn (FRET donor), resulting in an intermolecular FRET between donor and acceptor fluorophore (bottom panel). In contrast, F29 would be expected to show significantly lower binding and reduced or no FRET when Fyn is inactive (upper panel). (d) Sensing active Fyn in cells through binding-dependent FRET. Fluorescence spectral measurements from HEK-293T cells co-transfected with FRET-donor (WT or CA) (Fyn-mCerulean) alone OR along with a FRET-acceptor (either Fyn binder mVenus-F29 or the non-binding mutant, mVenus-F29P41A). Specifically, the normalized emission spectra of transiently transfected cells when excited at $435 \mathrm{~nm}$ (corresponding to donor mCerulean excitation) are shown. Spectra has been normalized relative to emission intensity at $475 \mathrm{~nm}$. Overall the spectra show that FRET ('sensitized' acceptor emission at $525 \mathrm{~nm}$ ) is greater for CA Fyn as compared to WT Fyn. No FRET signal is observed (sensitized $525 \mathrm{~nm}$ emission) with donor alone or non-binding mutant F29-P41A. Left inset figure shows that binding and increase in FRET efficiency is saturable. Using raw fluorescent intensity values the FRET efficiency was calculated as per the equation $\mathrm{Fa} /(\mathrm{Fa}+\mathrm{Fd})$, where $\mathrm{Fa}$ is acceptor and $\mathrm{Fd}$ is donor raw fluorescence intensity respectively. Increasing the amount of labeled binder (mVenus-F29/acceptor) in cells while maintaining Fyn (donor) levels constant causes an increase in FRET till saturation is reached (blue closed squares), indicating binding dependent FRET. From three independent experiments, a nonlinear best fit was plotted $\left(\mathrm{R}^{2}=0.97\right)$ using GraphPad Prism (blue, dotted line). In contrast, acceptorlabelled non-binder control (F29 P41A mutant) shows little or no FRET (red open symbol) showing FRET response is binding dependent and specific. Right inset figure shows a bar plot of FRET efficiency comparing WT and CA Fyn for a 1:2 donor:acceptor molar ratio. From three such independent experiments, Data was compared using Student's unpaired one-tailed t-test $(* * * p<0.0001)$ on GraphPad Prism. Values are mean \pm s.e.m.(e) Membrane tagging of F29 increases biosensor sensitivity and FRET efficiency. (i) The emission spectra of cells co-transfected with Fyn (WT) and, either mVenus-F29 or myr-mVenus-F29 at a 1:4 donor:acceptor molar ratio on $435 \mathrm{~nm}$ excitation are shown. Spectra for corresponding non-binding controls (mVenus-F29P41A with and without myristoyl tags) are also shown. (ii) Box-andWhisker plot showing observed FRET efficiency in cells when myristoyl-tag is added to the FRET-acceptor (mVenus-F29) or its non-binding control mutant P41A. Data from four such independent experiments were analyzed using Student's unpaired onetailed t-test on GraphPad Prism (respective $p$ values are indicated on panel). 

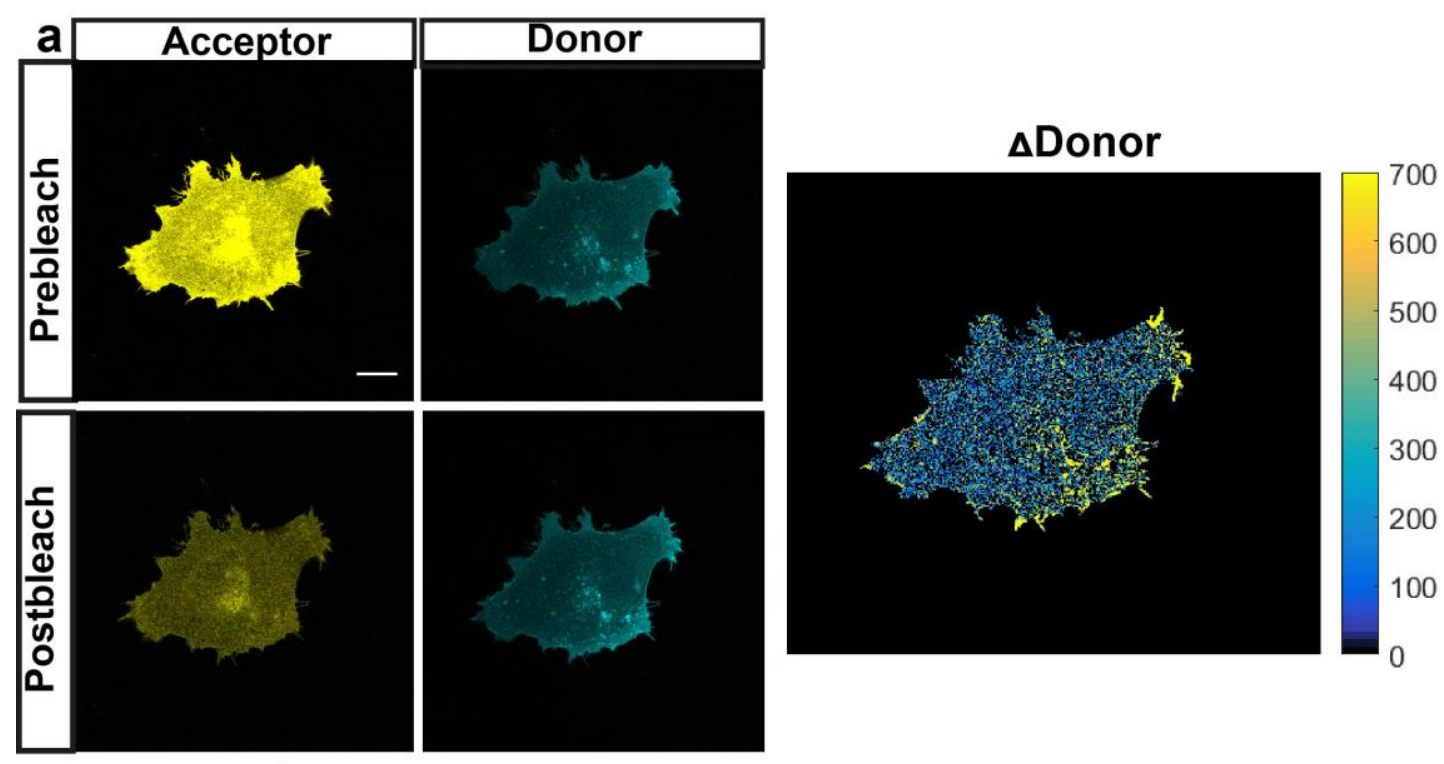

b

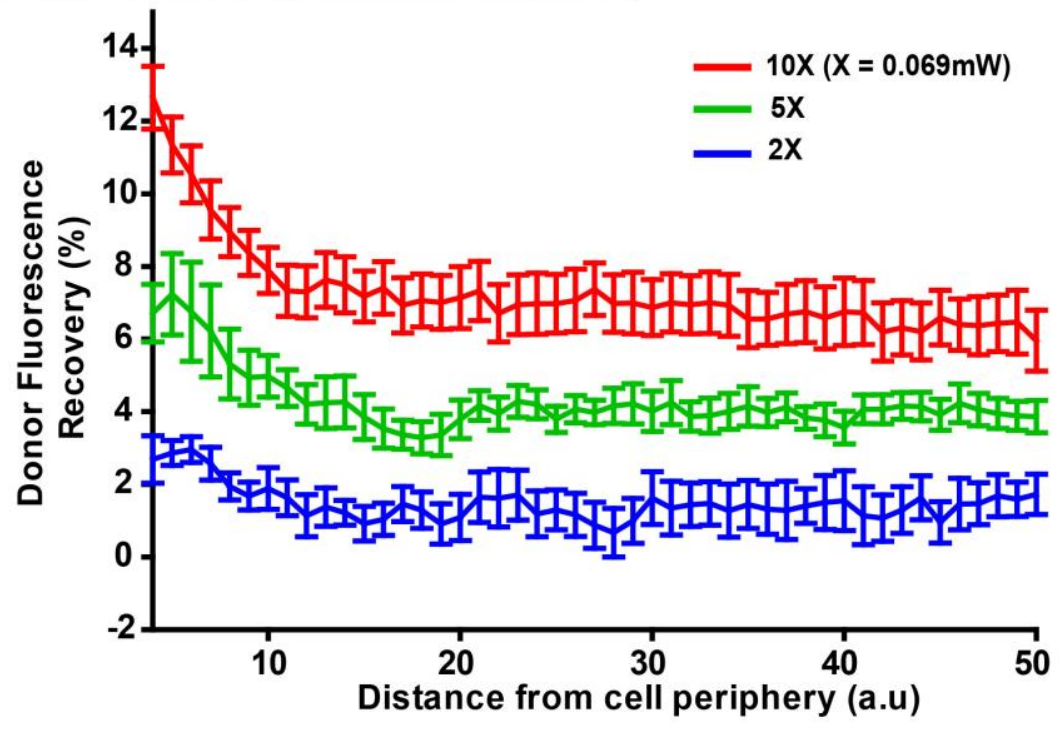

C

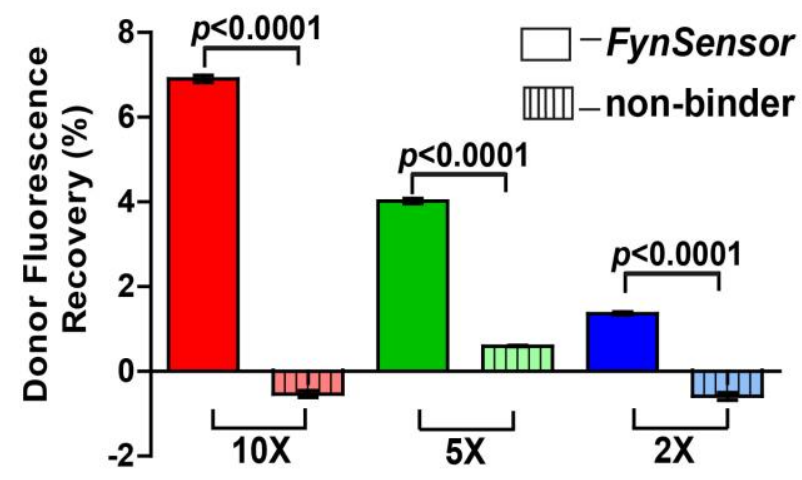

Bleaching dose $(\mathrm{mW})$

Fig. 5: FynSensor allows direct visualization of spatially localized active Fyn in cells.

Acceptor photo-bleaching (APB) experiment for visualizing active Fyn in U2OS cells co-expressing FRET donor (Fyn WT) and acceptor (myr-mVenus-F29) FynSensor constructs. (a) APB shows cellular FRET and localized Fyn activity. Representative figure shows donor and acceptor fluorescence images, prior to and after laser based 
bleaching of acceptor with $514 \mathrm{~nm}$ laser pulse. Also shown is the corresponding $\Delta$ Donor image (see methods) (Scale bar $=10 \mu \mathrm{m}$ ). APB experiments reveal areas showing greater recovery of donor fluorescence after APB indicating spatially localized Fyn activity (b) APB-induced recovery of donor fluorescence is greater at the cell periphery and sensitive to bleaching light dosage. Percentage recovery of donor fluorescence in FynSensor expressing cells, after acceptor photobleaching at different light dosage (using Equation 1, see methods), plotted as a function of distance from the cell edge. Extent of recovery also shown at different light dosage. Bleaching is carried out for 10 seconds using the $514 \mathrm{~nm}$ laser line at $2 \mathrm{X}, 5 \mathrm{X}$ and $10 \mathrm{X}$ doses $(\mathrm{X}=0.069 \mathrm{~mW})$. Lines represent mean of $n=14$ cells for $10 X, n=9$ cells for $5 X, n=7$ cells for $2 X$, respectively. Values are mean \pm s.e.m. (c) FRET signal measured through the APB method is specific (binding dependent) as well as sensitive to APB light doses. Cumulative APB-induced percentage recovery of donor fluorescence observed at the cell periphery in FynSensor expressing cells (as in b), plotted as a function of light dosage (solid bars). Also, shown extent of recovery when donor-labeled Fyn is used with acceptor-labeled non-binding P41A mutant of F29 (dashed bars). Extent of recovery with non-binding control is minimal and significantly lower than observed for F29 FynSensor. Bars represent mean of $n=14$ cells for $10 \mathrm{X}, \mathrm{n}=9$ cells for $5 \mathrm{X}$ and $\mathrm{n}=7$ cells for $2 \mathrm{X}$ when binder/F29 is used and $\mathrm{n}=14$ cells for $10 \mathrm{X}, \mathrm{n}=10$ cells for $5 \mathrm{X}$ and $\mathrm{n}=8$ cells for $2 \mathrm{X}$ when non-binder/F29P41A is used, respectively. Student's unpaired one-tailed t-test was used to determine the p-value. Graph was made using GraphPad Prism. Values are mean \pm s.e.m. 
a (i)

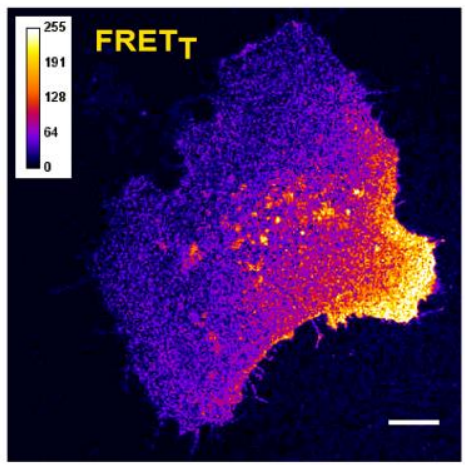

(ii)

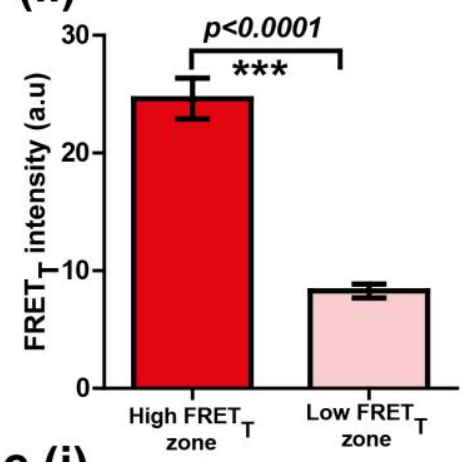

c (i)
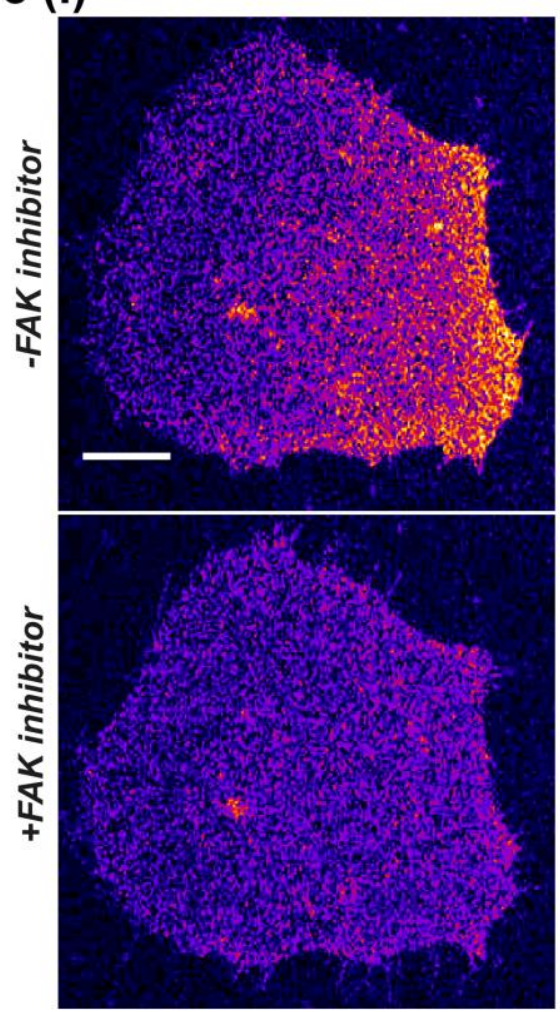

\section{U2OS:FynSensor}

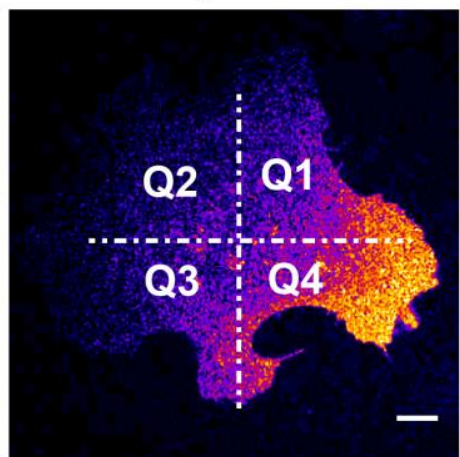

b (i) U2OS:non-binder

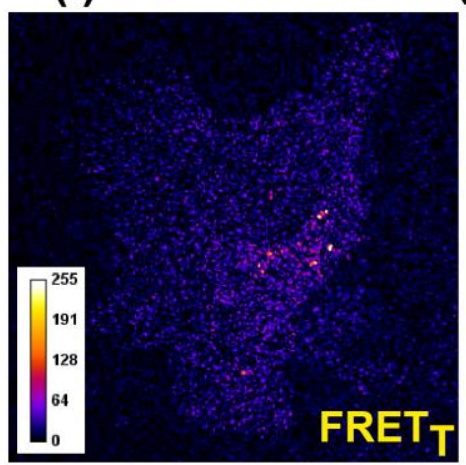

(ii)

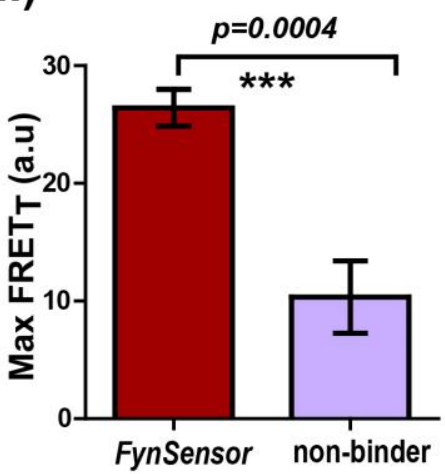

(ii) $901-$ - FRETT intensity

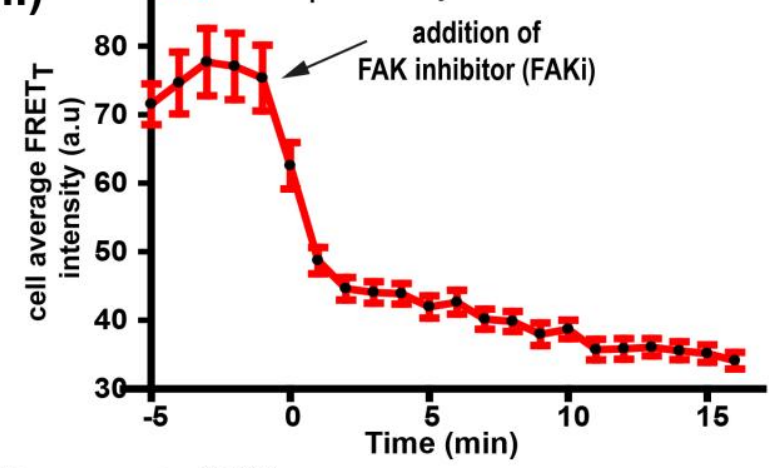

(iii)

(iv)
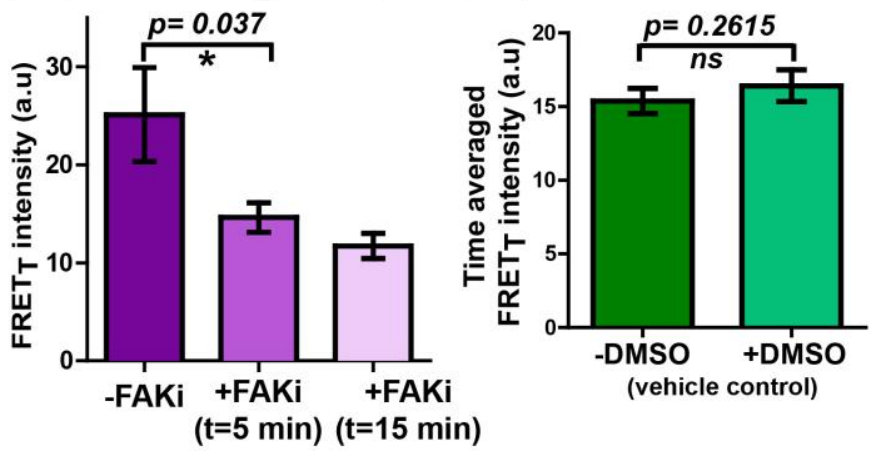

Fig. 6: FynSensor reveals integrin-dependent Fyn activity to be spatially compartmentalized.

(a) Spontaneous formation of zones enriched in active Fyn is seen in serum starved cells plated on fibronectin (FN). Representative confocal fluorescence micrographs showing sensitized emission (total FRET: FRET $_{\mathrm{T}}$ ) levels indicative of active Fyn in 
serum starved-U2OS cells expressing FynSensor (Scale bar $=10 \mu \mathrm{m}$ ). For quantitative image analysis of compartmentalized Fyn activity, we have divided the cell into quadrants labelled as Q1-Q4 as shown. (ii) Bar graph comparing FynSensor FRET levels in distinct cellular zones. The mean of Max-FRET ${ }_{\mathrm{T}}-\mathrm{HFQ}$ (HFQ: high-FRET quadrant, Q4 in panel i) and Max-FRET ${ }_{\mathrm{T}}-\mathrm{LFQ}$ (LFQ: low-FRET quadrant, Q2 in i) values (see methods) are plotted. Values are mean \pm s.e.m. Student's paired one-tailed $\mathrm{t}$-test has been used to determine the p-value ( $\mathrm{n}=37$ cells). (b) FynSensor FRET readout is dependent on F29 binding Fyn, with the non-binding control mutant of F29 (P41A) showing no or significantly reduced $\mathrm{FRET}_{\mathrm{T}}$ signal compared to FynSensor. (i) Confocal fluorescence micrographs showing sensitized emission (total FRET: FRET $_{\mathrm{T}}$ ) levels for cells expressing mCer-Fyn and non-binding control binder mVenus-F29 P41A. (ii) Comparison of $\mathrm{FRET}_{\mathrm{T}}$ levels for FynSensor with non-binding control. The bar graph shows the average maximum FRET $_{\mathrm{T}}$ obtained (Max-FRET $\left.\mathrm{T}_{\mathrm{T}} \mathrm{HFQ}\right)$ for non-binder ( $\mathrm{n}=5$ cells) and FynSensor ( $\mathrm{n}=37$ cells). Values are mean \pm s.e.m. Student's unpaired one tailed-t-test was used to determine the p-value. (c) Fyn activity levels are sensitive to inhibition of focal adhesion kinase (FAK), a known mediator of integrin signaling. (i) Confocal fluorescence micrographs showing FynSensor FRET $_{\mathrm{T}}$ levels before and after treatment with $10 \mu \mathrm{M}$ of FAK inhibitor (PF 562271). Loss of basal Fyn activity is observed in cells when treated with inhibitor but not with DMSO (vehicle control). (ii) Time course of Fyn activity in response to FAK inhibition. Average fluorescence intensity profile $\left(\mathrm{FRET}_{\mathrm{T}}\right)$ of FynSensor cells $(\mathrm{n}=5)$ over time, before and after addition of FAK inhibitor. (Scale bar $=10 \mu \mathrm{m}$ ). (iii) Quantifying FynSensor FRET on FAK inhibition. Bar graph shows the average $\mathrm{FRET}_{\mathrm{T}}$ intensity of cells before FAK inhibitor treatment (-FAKi), 5 mins after FAKi treatment and 15 mins after FAKi treatment ( $n=5$ cells). Values are mean \pm s.e.m. Student's paired one tailed-t-test was used to determine the p-value. (iv) FAK-inhibitor induced reduction in FynSensor FRET levels is NOT seen with vehicle control. Bar graph shows time-averaged, mean cell FRET $_{\mathrm{T}}$ intensity before and after treatment with the vehicle control ( $n=8$ cells). Values are mean \pm s.e.m. Student's paired one tailed-t-test was used to determine the p-value. 
a (i)

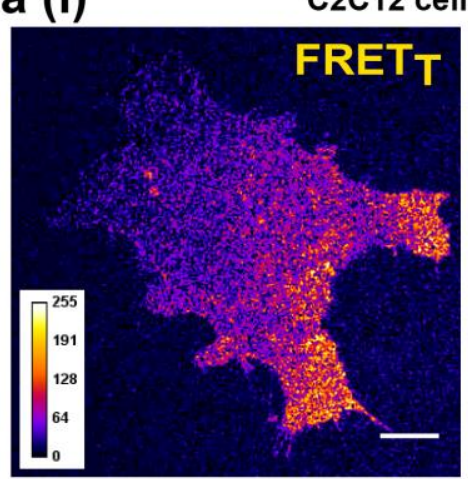

b (i)

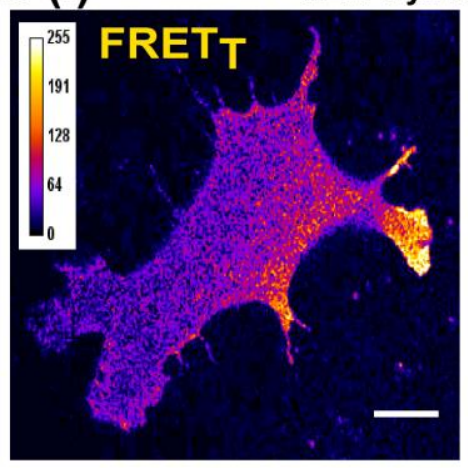

C
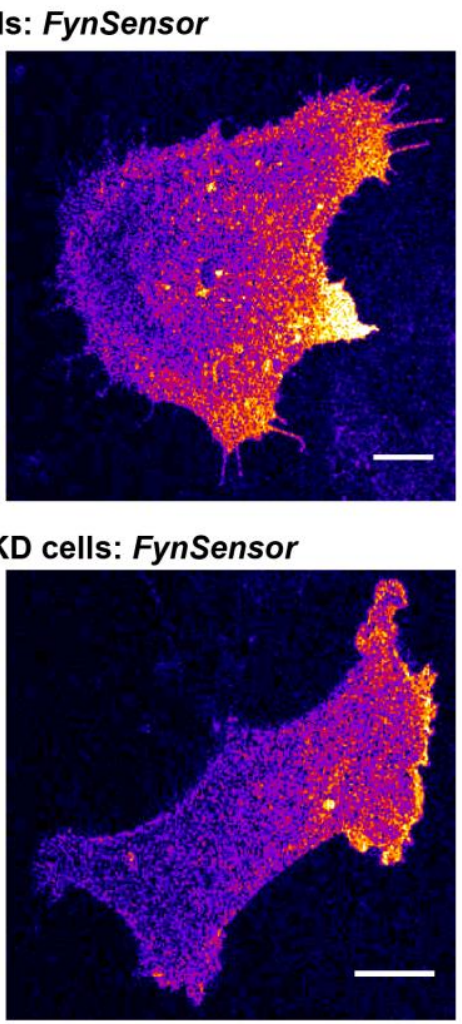

(ii)

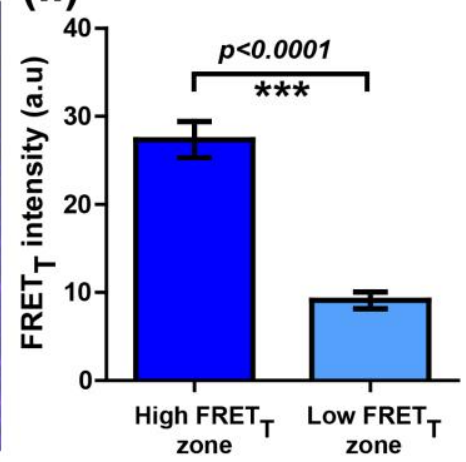

(ii)

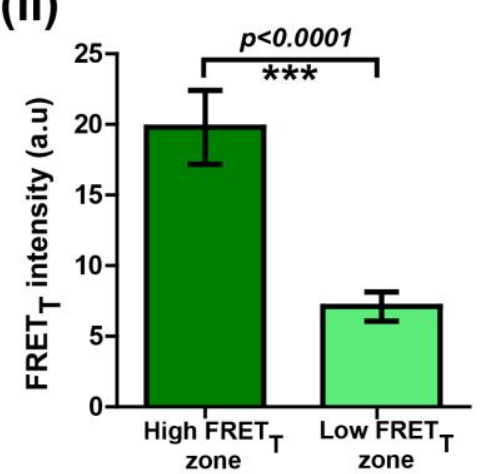
zone

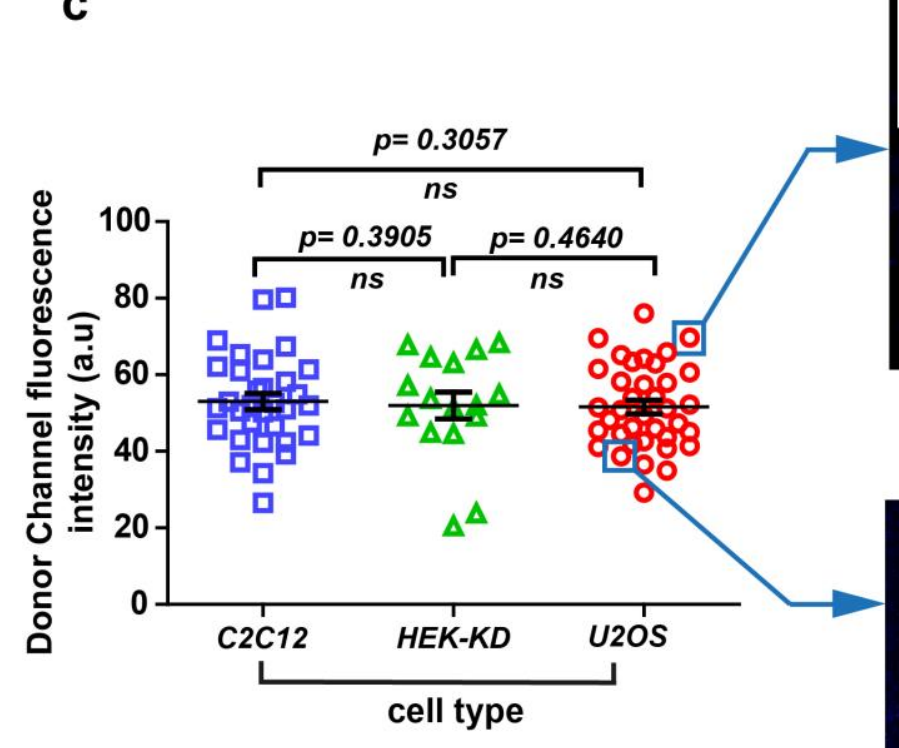

-

Fig. 7: Conserved spatial pattern of Fyn activity in distinct cell-types.

(a) FynSensor in mouse myoblast $\mathrm{C} 2 \mathrm{C} 12$ cells reveals spontaneously compartmentalized Fyn activity, similar to that observed in U2OS cells. (i) Representative confocal fluorescence micrographs of serum-starved cells showing 
intracellular zones enriched with active Fyn when plated on FN (Scale bar $=10 \mu \mathrm{m})$. Quantitative image analysis, as previously described, yielded zones of high FRET $_{\mathrm{T}}$ and low FRET T. (ii) Bar graph comparing FynSensor FRET levels in distinct cellular zones. Graph compares the mean of Max-FRET ${ }_{\mathrm{T}}$-HFQ and Max-FRET $\mathrm{T}_{\mathrm{T}}$-LFQ in serumstarved cells. Values are mean \pm s.e.m. Student's paired one-tailed t-test has been used to determine the p-value ( $\mathrm{n}=32$ cells). (b) FynSensor imaging reveals spatially localized Fyn activity patterns when FynSensor is ectopically expressed in HEK293T Fyn-knockdown cells. (i) Confocal micrographs show zones of high and low FRET $_{T}$, showing spatially localized Fyn activity, similar to that seen with U2OS and C2C12 myoblasts. (ii) Bar graphs comparing the mean of Max-FRET $\mathrm{T}_{\mathrm{T}} \mathrm{HFQ}$ and Max-FRET $\mathrm{T}^{-}$ LFQ in serum-starved cells confirm differential FRET levels across intracellular zones. Values are mean \pm s.e.m. Student's paired one-tailed t-test has been used to determine the $\mathrm{p}$-value $(\mathrm{n}=16$ cells). (c) Spatial patterns of locally-enhanced Fyn activity revealed by FynSensor imaging are unaffected by FynSensor expression levels. FynSensor imaging reveals locally enhanced, compartmentalized Fyn activity independent of sensor expression levels. To assess the effect of kinase expression on the FRET patterns observed in all the distinct cell types, we quantified the donor (m-Cerulean) fluorescence intensity for all the cells used in our study. Scatter-plot comparing donor fluorescence across different cell types, $\mathrm{n}=32$ cells for C2C12, 37 for U2OS and 16 for HEK-KD cells. Mean \pm s.e.m is denoted. Student's unpaired one-tailed t-test has been used to determine the p-value. Representative cells from the U2OS data set, having either relatively 'high' (above the mean value of $\mathbf{5 1 . 6 1} \pm \mathbf{1 . 8 0 5}$ fluorescence intensity units, denoted by teal box) or 'low' (below the mean value, teal box) FynSensor expression (donor/m-Cer fluorescence intensities), show very similar spatially compartmentalized Fyn activity pattern, suggesting that the activity patterns observed in different cells are not subject to expression artifacts. 

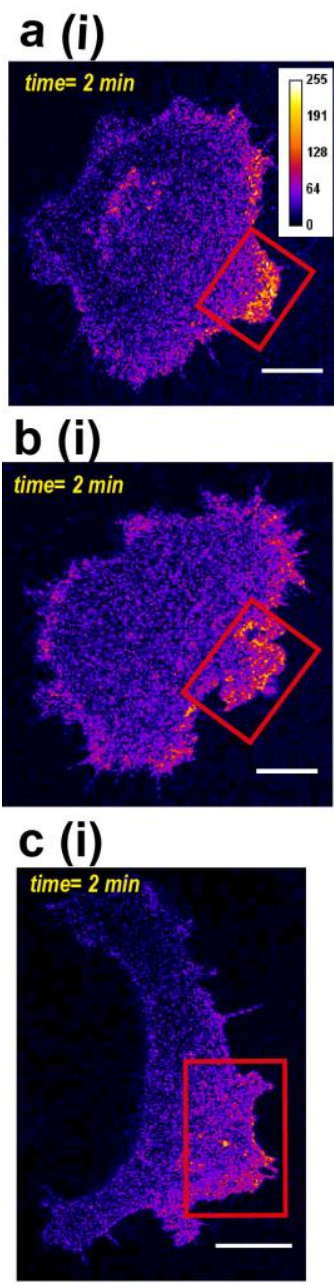

d

\section{U2OS cells}

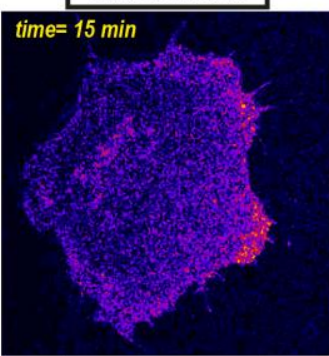

C2C12 cells

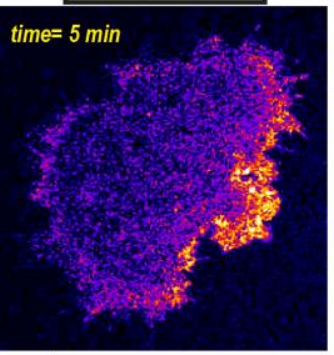

HEK- Fyn KD cells

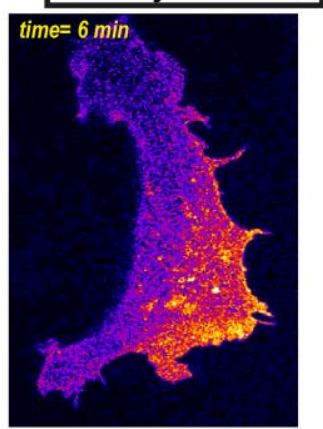

e (i)
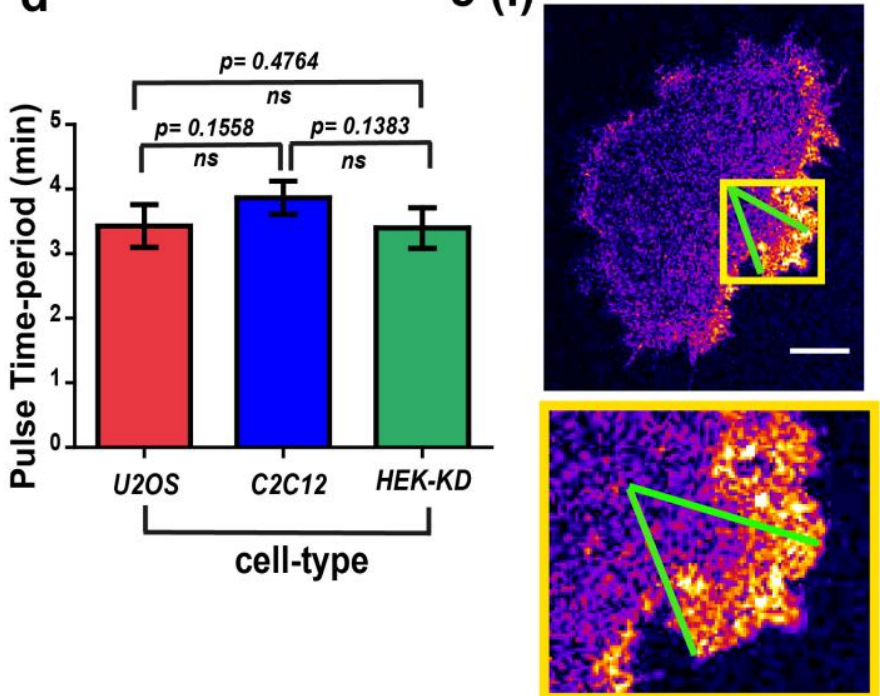

(ii)
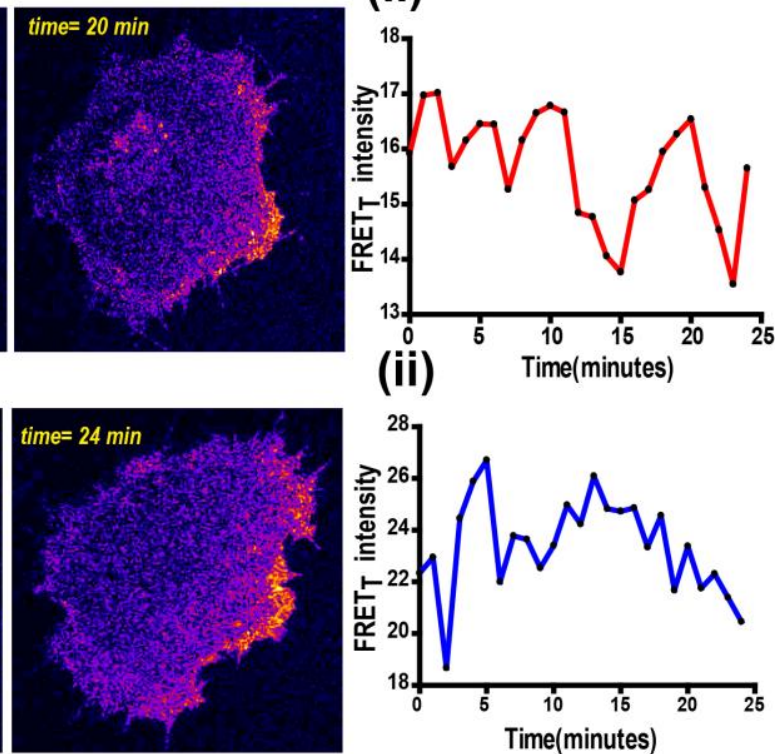

(ii)
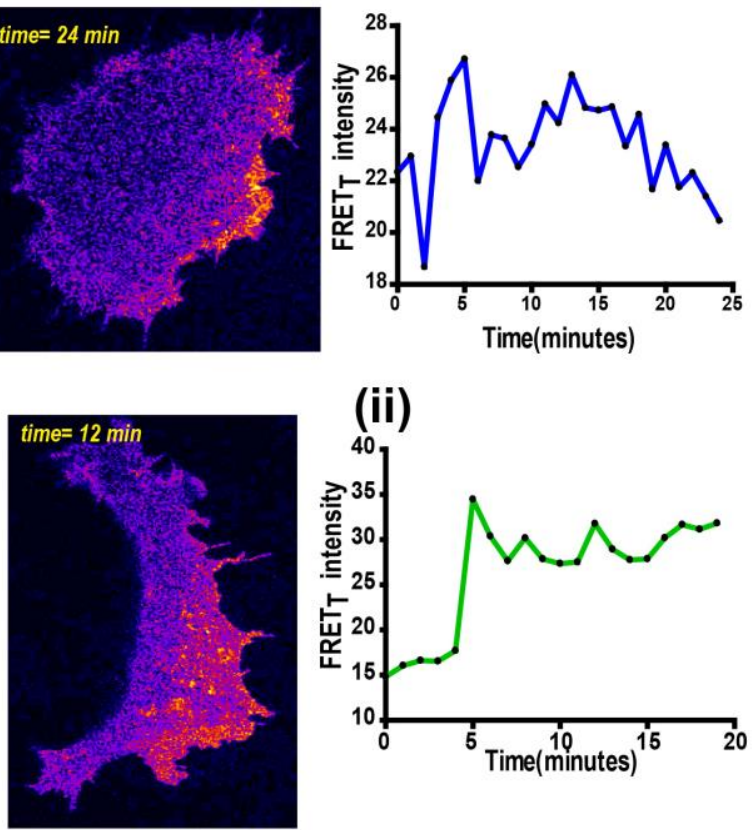

(ii)
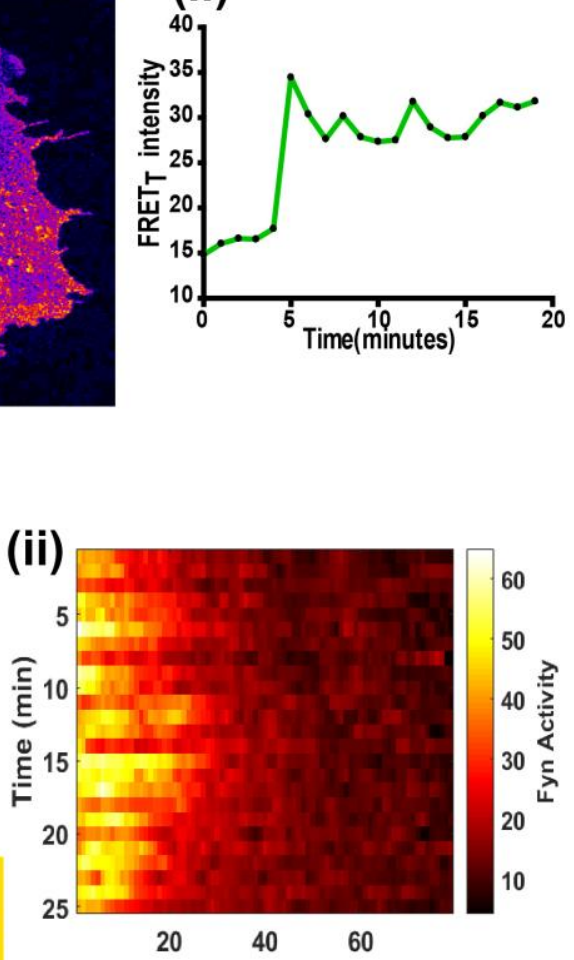

Distance from cell periphery(a.u)

Fig 8: FynSensor imaging reveals spatially-compartmentalized temporal bursts of kinase activity across distinct cell types.

(a) A striking feature revealed by FynSensor is the pulsatile nature of Fyn activation. (i) Representative confocal micrographs of a U2OS cell at different time points after being plated on FN, as indicated, reveal significant oscillations in the $\mathrm{FRET}_{\mathrm{T}}$ intensity (area marked by red box) (Scale bar $=10 \mu \mathrm{m}$ ). (ii) Time-traces of the $\mathrm{FRET}_{\mathrm{T}}$ signal showing temporal pulses of Fyn activity. (b) FynSensor imaging in C2C12 mouse 
myoblast cells, a cell-type distinct from the U2OS human osteosarcoma cells, also show an oscillating Fyn activity profile. (i) Confocal micrographs from a $\mathrm{C} 2 \mathrm{C} 12$ cell transiently expressing FynSensor, at different time-points post FN plating, shows temporal bursts of kinase activity (area marked by red box). (ii) Time-trace showing the pulsatile temporal pattern in FRET ${ }_{\mathrm{T}}$ signal in the cell shown. (c) FynSensor imaging reveals pulsatile activity patterns in Fyn knock-down cells that have been 'rescued' with labeled Fyn (m-Cer-Fyn). Fyn activity pulses are similar to those observed in U2OS and $\mathrm{C} 2 \mathrm{C} 12$, showing temporal patterns are robust and conserved. (i) Representative cell confocal micrograph of RNAi-Fyn knockdown HEK-293 (KD) cells expressing FynSensor show temporal oscillations in FRET $_{\mathrm{T}}$ levels (area marked by red box). (ii) Time-trace of $\mathrm{FRET}_{\mathrm{T}}$ signal from individual cell revealing pulsatile Fyn signal. (d) Oscillatory/pulsatile behavior in levels of active Fyn in the different cell-types studied reveal a consistent major time period. Plotted here is the mean of the dominant timeperiod of FynSensor FRET pulses in U2OS C2C12 and HEK-KD cells expressing the sensor, determined through power spectrum density (PSD) analysis on the time-traces of single cell quadrant averaged total FRET. The PSD analysis reveals the mean of dominant time-period to be $\sim 3.5$ minutes in distinct cell types. $\mathrm{N}=37$ for U2OS, 32 for C2C12 and 16 for HEK-KD cells. Values are mean \pm s.e.m. Student's two-tailed unpaired t-test has been used to determine the p-value. See methods for details of the analysis. (e) Pulsatile Fyn activity is spatially-constrained. Intracellular zones of elevated Fyn activity also show clear oscillations over time. (i) Confocal fluorescence micrograph of a $\mathrm{C} 2 \mathrm{C} 12$ cell shows a zone of high FRET signal (indicated by yellow box). For plotting temporal oscillations over distance from edge, an arc (of arbitrary length units) was suspended on the membrane encompassing this zone. A smaller sector was then created on the cell (indicated by green lines). (ii) Plots shows a heat map of FRET $_{\mathrm{T}}$ intensity values in this sector, over time, as a function of distance from the cell- membrane. Data reveals that active kinase to be spatially constrained and oscillating over time. See methods for details of the analysis. 
a (i)

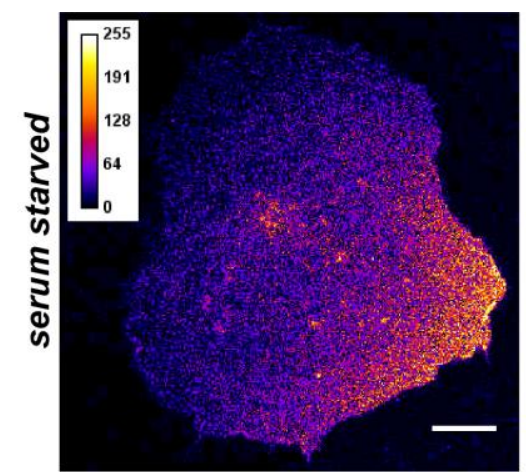

b (i)

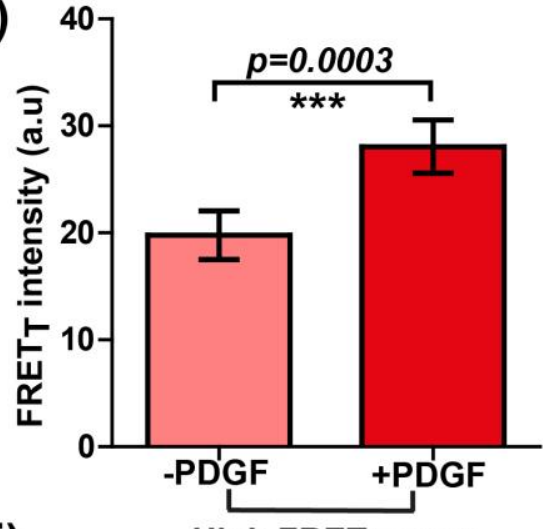

C (i)

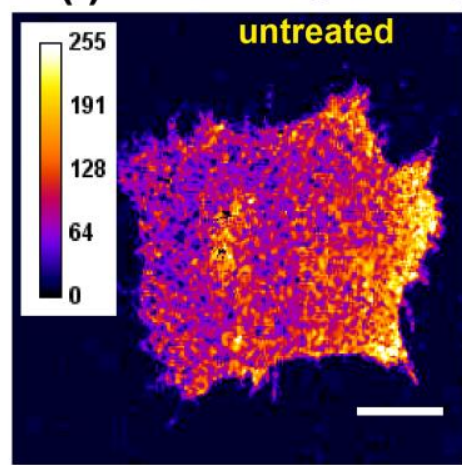

(ii)
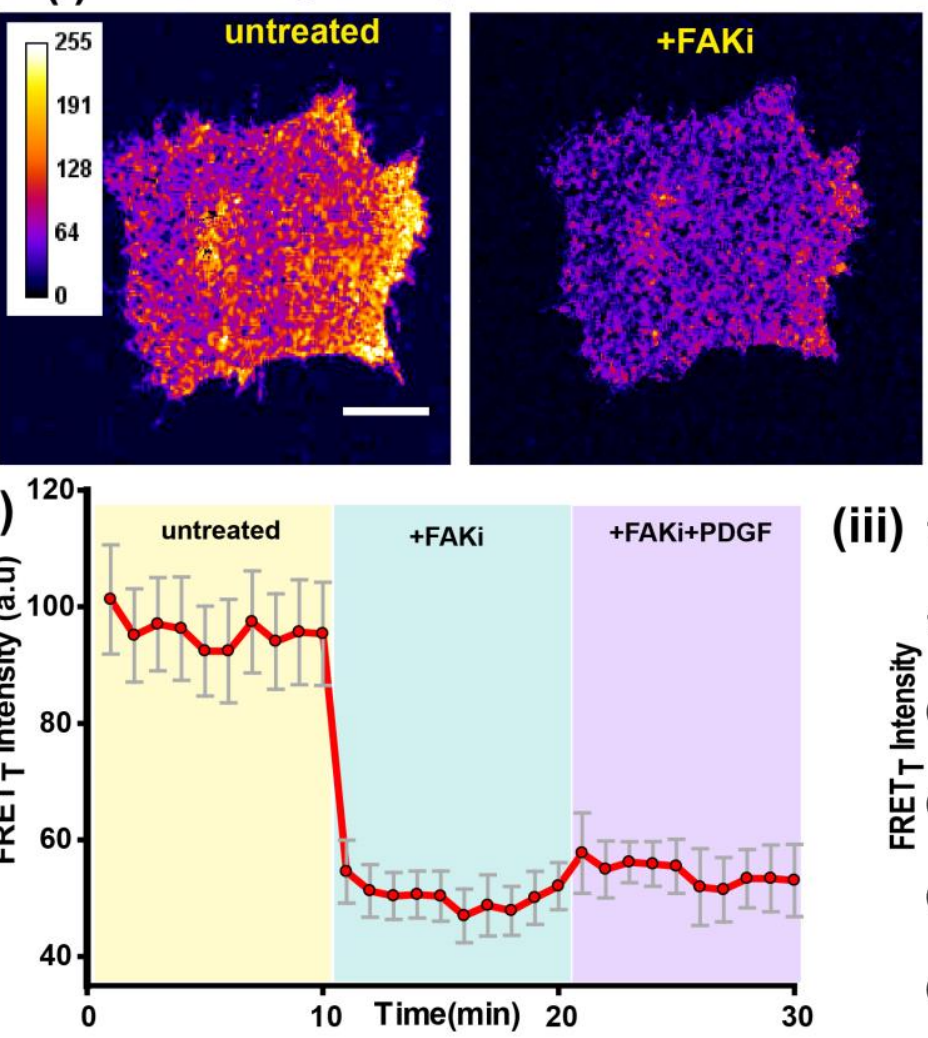

(ii)

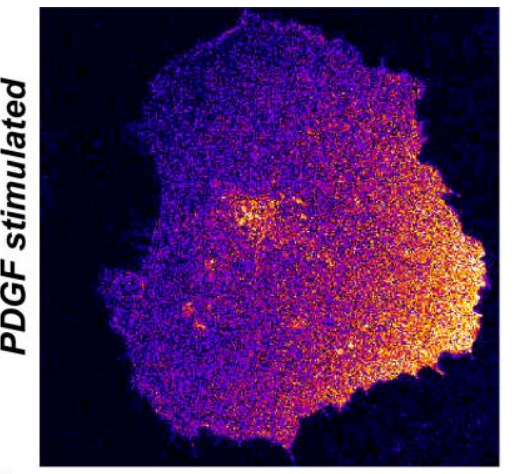

(ii)
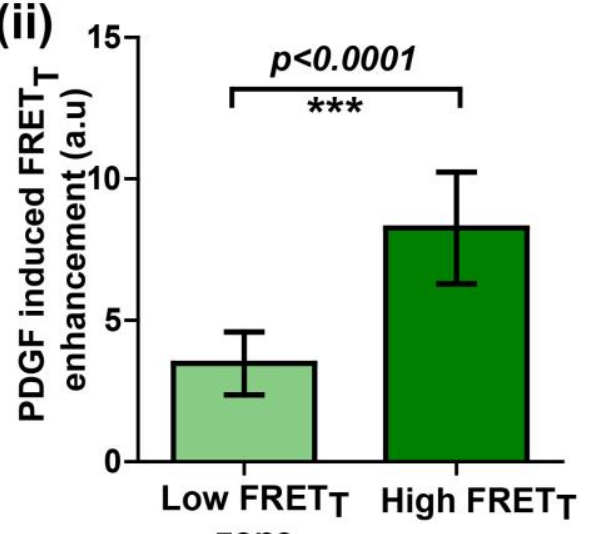

zone

zone
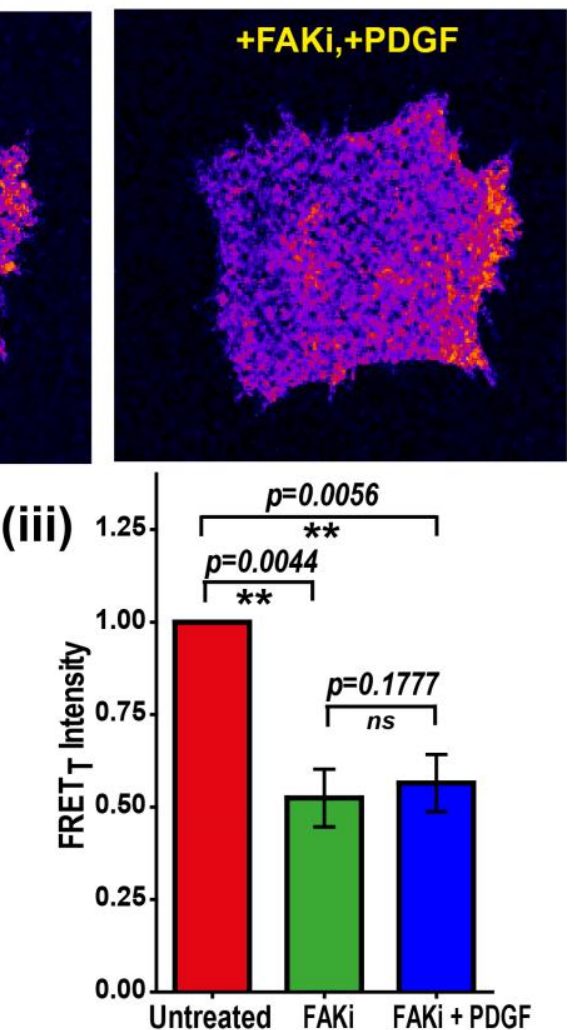

Fig 9: Integrin-dependent, spatially compartmentalized, pulsatile Fyn activity can be modulated by growth factor suggesting signaling crosstalk.

(a) Spontaneously formed zones of active Fyn in serum-starved cells plated on FN can be further modulated by Platelet-derived growth factor (PDGF). Representative confocal fluorescence micrographs showing sensitized emission $\left(\mathrm{FRET}_{\mathrm{T}}\right)$ levels 
indicative of active Fyn in serum starved (i) and PDGF stimulated U2OS cells (ii) (Scale bar $=10 \mu \mathrm{m})$. (b) Quadrant analysis of cells reveal that on a global PDGF stimulation of serum-starved cells, the increase in Fyn activity observed was spatially localized (i) Bar graph comparing the mean of Max-FRET $\mathrm{T}_{\mathrm{T}}-\mathrm{HFQ}$ before and after addition of PDGF $(10 \mathrm{ng} / \mathrm{ml})$ indicates that the FRET $_{\mathrm{T}}$ intensity increase in the high FRET zone on addition of PDGF. Values are mean \pm s.e.m. Student's paired one-tailed $\mathrm{t}$-test has been used to determine the $\mathrm{p}$-value ( $\mathrm{n}=18$ cells). (ii) PDGF-induced enhancement in Fyn activity is greater in pre-activated intracellular zones. Bar graph show the difference in the mean intensities of the maximum FRET $_{\mathrm{T}}\left(\mathrm{Max}_{\left.-\mathrm{FRET}_{\mathrm{T}}\right)}\right.$ obtained before and after PDGF addition for both 'high FRET' as well as 'low FRET' quadrants/zones across single cells. Quadrants were designated as 'HFQ' or 'LFQ' in FN-plated cells, prior to PDGF stimulation $(\mathrm{n}=18$ cells). Values are mean \pm s.e.m. Student's paired one-tailed t-test has been used to determine the p-value. (c) Focal adhesion kinase (FAK) activity not only regulates Fyn activity but is required for the modulation/enhancement of Fyn activity by platelet derived growth factor. (i) Confocal fluorescence micrographs showing sensitized emission (FRET ${ }_{\mathrm{T}}$ ) levels in untreated/serum starved cells, cells treated with FAK inhibitor (FAKi, PF-562271 as well as cell pre-treated with FAK inhibitor prior to stimulation with PDGF. (Scale bar= $10 \mu \mathrm{m}$ ). FAK inhibition attenuates FRET levels (active Fyn levels) in FN-plated serumstarved cells AND abolishes the enhancement and modulation of Fyn activity by PDGF. FAK-i treated cells fail to respond to PDGF as measured through FynSensor FRET levels. (ii) The mean $\mathrm{FRET}_{\mathrm{T}}$ intensity profile for the high $\mathrm{FRET}_{\mathrm{T}}$ quadrant in 4 cells plotted over time. Also, marked in color are experimental condition or the treatments; serum starved cells (yellow, $\mathrm{t}=0-10 \mathrm{mins}$ ), $+\mathrm{FAKi}$ (cyan, $\mathrm{t}=10-20$ mins) AND + FAKi+PDGF (lavender, $\mathrm{t}=20-30$ mins). Values are mean \pm s.e.m. (iii) Whole cell FRET $_{\mathrm{T}}$ data from all the cells $(\mathrm{n}=4)$ for untreated cells, $+\mathrm{FAKi}$, and $+\mathrm{FAKi}+\mathrm{PDGF}$. The mean intensity value of untreated cells has been used to normalize the data. Student's two-tailed paired t-test has been used to determine the p-value. Values are mean \pm s.e.m. Data show no significant enhancement is seen in Fyn activity by PDGF stimulation, if cells are pre-treated with FAKi. 


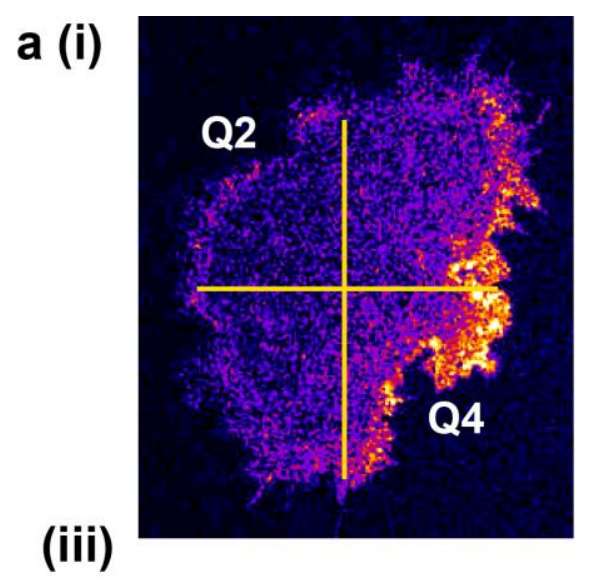

(ii)
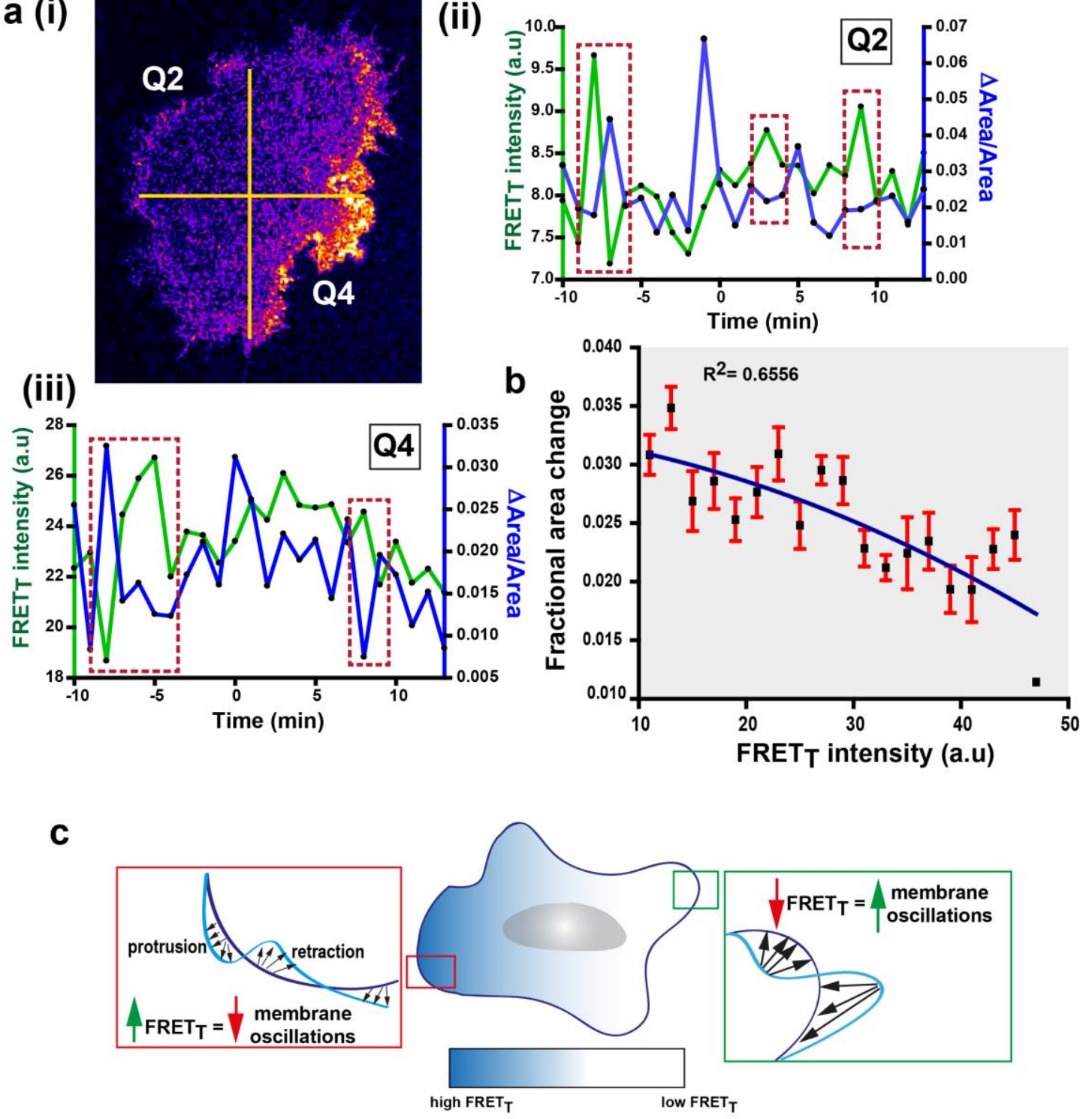

Fig 10: Fyn activity couples to cellular morphodynamics.

(a) Fyn activity correlates with reduced amplitudes of membrane protrusion-retraction as revealed by simultaneous measurement of cell area, perimeter and FynSensor FRET $T$ levels. (i) Graph plotted here shows $\mathrm{FRET}_{\mathrm{T}}$ intensity profile and the change in area ( $\triangle$ Area/Area) for two quadrants, Q2 (i) and Q4 (ii) in a representative confocal micrograph. Here FRET $_{T}$ levels appear to negatively correlate with changes in area change (see red dotted boxes). (b) Graph shows the cumulative time-averaged change in area, plotted against FRET $_{T}$ intensity for $\mathrm{C} 2 \mathrm{C} 12$ cells $\left(\mathrm{n}=32\right.$ cells). We find $\mathrm{FRET}_{\mathrm{T}}$ levels and area change are inversely correlated with an $\mathrm{R}^{2}$ value $=0.6556$ (Plot fitted using the equation $\mathbf{f}=\mathbf{y} \mathbf{0}+\mathbf{a}^{*} \mathbf{x}+\mathbf{b}^{*} \mathbf{x} \mathbf{2}$ ). Values are mean \pm s.e.m. (c) An illustration of Fyn activity being negatively correlated to membrane morphodynamics wherein, cellular zones showing higher Fyn activity (high FRET $_{\mathrm{T}}$ ) show reduced overall area changes (lesser constitutive protrusion/retraction cycles). 


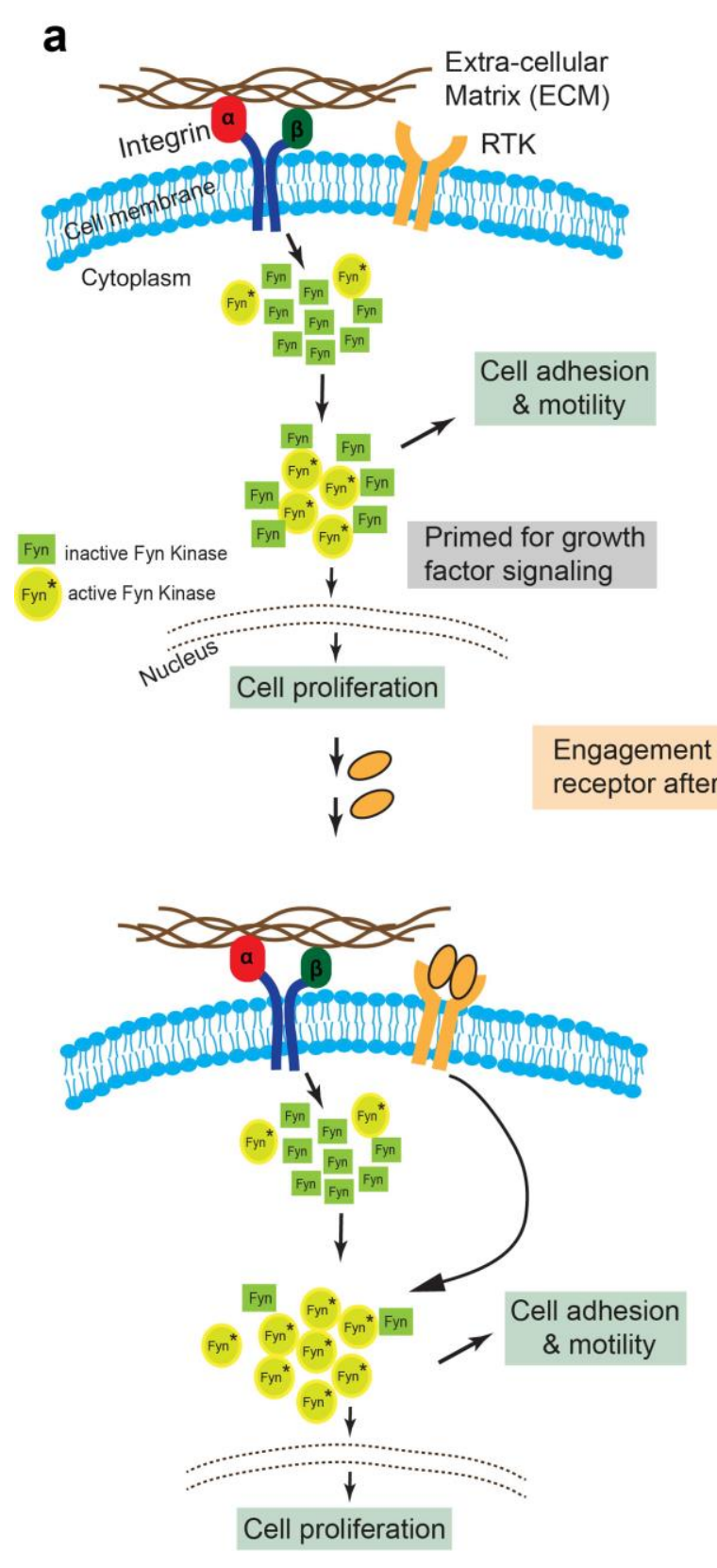

b

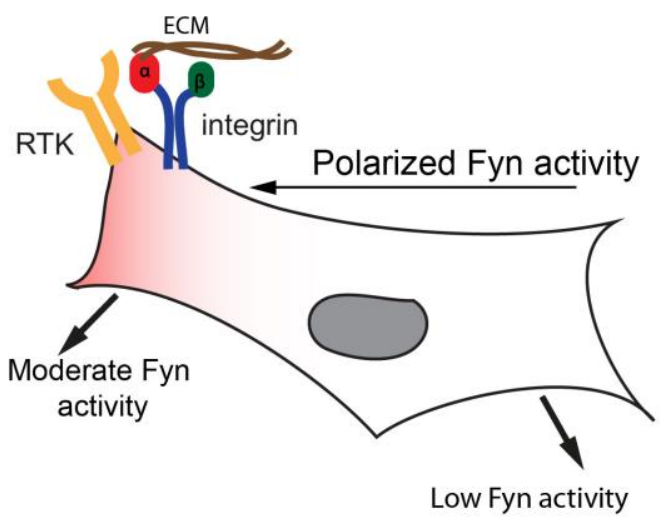

Fig. 11: Model representing how Fyn activity helps mediate spatio-temporally localized signalling crosstalk.

(a) Src kinases have been long known to associate with the integrin receptor and growth factor receptor (RTK) signaling, thereby placing these kinases at nodal spots for signal integration. We provide the first visual proof for Fyn's involvement in integrating signals from these two receptor classes and see that the cell is highly polarized with respect to Fyn kinase activity. FynSensor shows that integrin receptor engagement results in formation of "zones" of active Fyn in the cell, causing the cell to become polarized with respect to the activity of the kinase, even in serum-starved, FN-plated cells. PDGF stimulation further increases Fyn activity preferentially in these presensitized regions only, thereby strengthening the spatially localized response. (b) Spatio-temporally localized Fyn activity; specifically, set-up through signal integration offers a quantitative picture of how Fyn activity may help regulate cell fate in certain 
bioRxiv preprint doi: https://doi.org/10.1101/726133; this version posted August 5, 2019. The copyright holder for this preprint (which was

not certified by peer review) is the author/funder, who has granted bioRxiv a license to display the preprint in perpetuity. It is made available under aCC-BY-NC-ND 4.0 International license.

situations. Fyn is known to be crucial for context-dependent cell migration and cell state switching in normal as well malignant cells, therefore, a spatio-temporally polarized response may be important for setting up cell output, especially for processes like that of directional cell migration and adhesion dependent cell switching. Pulsatile Fyn activity further suggests pre-organized signaling clusters with negative and positive feedback loops sensitive to both integrin and growth factor signaling. 


\section{References}

1 Cai, D. et al. Mechanical feedback through E-cadherin promotes direction sensing during collective cell migration. Cell 157, 1146-1159, doi:10.1016/j.cell.2014.03.045 (2014).

2 Devreotes, P. N. et al. Excitable Signal Transduction Networks in Directed Cell Migration. Annual review of cell and developmental biology, doi:10.1146/annurev-cellbio-100616-060739 (2017).

3 Ridley, A. J. et al. Cell migration: integrating signals from front to back. Science 302, 1704-1709, doi:10.1126/science.1092053 (2003).

4 Depry, C., Mehta, S., Li, R. \& Zhang, J. Visualization of Compartmentalized Kinase Activity Dynamics Using Adaptable BimKARs. Chemistry \& biology 22, 1470-1479, doi:10.1016/j.chembiol.2015.10.004 (2015).

5 Gulyani, A. et al. A biosensor generated via high-throughput screening quantifies cell edge Src dynamics. Nature chemical biology 7, 437-444, doi:10.1038/nchembio.585 (2011).

6 Komatsu, N. et al. Development of an optimized backbone of FRET biosensors for kinases and GTPases. Molecular biology of the cell 22, 4647-4656, doi:10.1091/mbc.E11-01-0072 (2011).

7 Abram, C. L. \& Courtneidge, S. A. Src family tyrosine kinases and growth factor signaling. Experimental cell research 254, 1-13, doi:10.1006/excr.1999.4732 (2000).

8 Giannone, G. \& Sheetz, M. P. Substrate rigidity and force define form through tyrosine phosphatase and kinase pathways. Trends in cell biology 16, 213-223, doi:10.1016/j.tcb.2006.02.005 (2006).

9 Grande-Garcia, A. et al. Caveolin-1 regulates cell polarization and directional migration through Src kinase and Rho GTPases. The Journal of cell biology 177, 683-694, doi:10.1083/jcb.200701006 (2007).

10 Parsons, S. J. \& Parsons, J. T. Src family kinases, key regulators of signal transduction. Oncogene 23, 7906-7909, doi:10.1038/sj.onc.1208160 (2004).

11 Thomas, S. M. \& Brugge, J. S. Cellular functions regulated by Src family kinases. Annual review of cell and developmental biology 13, 513-609, doi:10.1146/annurev.cellbio.13.1.513 (1997).

12 Chetty, S. et al. A Src inhibitor regulates the cell cycle of human pluripotent stem cells and improves directed differentiation. The Journal of cell biology 210, 12571268, doi:10.1083/jcb.201502035 (2015).

13 Gujral, T. S. et al. A noncanonical Frizzled2 pathway regulates epithelialmesenchymal transition and metastasis. Cell 159, 844-856, doi:10.1016/j.cell.2014.10.032 (2014).

14 Kim, L. C., Song, L. \& Haura, E. B. Src kinases as therapeutic targets for cancer. Nature reviews. Clinical oncology 6, 587-595, doi:10.1038/nrclinonc.2009.129 (2009).

15 Lewis-Tuffin, L. J. et al. Src family kinases differentially influence glioma growth and motility. Molecular oncology 9, 1783-1798, doi:10.1016/j.molonc.2015.06.001 (2015).

16 Nygaard, H. B., van Dyck, C. H. \& Strittmatter, S. M. Fyn kinase inhibition as a novel therapy for Alzheimer's disease. Alzheimer's research \& therapy 6, 8, doi:10.1186/alzrt238 (2014). 
17 Saad, F. Src as a therapeutic target in men with prostate cancer and bone metastases. BJU international 103, 434-440, doi:10.1111/j.1464410X.2008.08249.x (2009).

18 Timpson, P., Jones, G. E., Frame, M. C. \& Brunton, V. G. Coordination of cell polarization and migration by the Rho family GTPases requires Src tyrosine kinase activity. Current biology : CB 11, 1836-1846 (2001).

19 Zhang, S. et al. SRC family kinases as novel therapeutic targets to treat breast cancer brain metastases. Cancer research 73, 5764-5774, doi:10.1158/00085472.CAN-12-1803 (2013).

20 Zhang, X., Simerly, C., Hartnett, C., Schatten, G. \& Smithgall, T. E. Src-family tyrosine kinase activities are essential for differentiation of human embryonic stem cells. Stem cell research 13, 379-389, doi:10.1016/j.scr.2014.09.007 (2014).

21 Liao, X. et al. Visualization of Src and FAK activity during the differentiation process from HMSCs to osteoblasts. PloS one 7, e42709, doi:10.1371/journal.pone.0042709 (2012).

22 Ouyang, M., Sun, J., Chien, S. \& Wang, Y. Determination of hierarchical relationship of Src and $\mathrm{Rac}$ at subcellular locations with FRET biosensors. Proceedings of the National Academy of Sciences of the United States of America 105, 14353-14358, doi:10.1073/pnas.0807537105 (2008).

23 Ouyang, M. et al. Sensitive FRET Biosensor Reveals Fyn Kinase Regulation by Submembrane Localization. ACS sensors 4, 76-86, doi:10.1021/acssensors.8b00896 (2019).

24 Seong, J., Lu, S. \& Wang, Y. Live Cell Imaging of Src/FAK Signaling by FRET. Cellular and molecular bioengineering 2, 138-147, doi:10.1007/s12195-0110161-3 (2011).

25 Wang, Y. et al. Visualizing the mechanical activation of Src. Nature 434, 10401045, doi:10.1038/nature03469 (2005).

26 Regot, S., Hughey, J. J., Bajar, B. T., Carrasco, S. \& Covert, M. W. Highsensitivity measurements of multiple kinase activities in live single cells. Cell 157, 1724-1734, doi:10.1016/j.cell.2014.04.039 (2014).

27 Kuo, G., Arnaud, L., Kronstad-O'Brien, P. \& Cooper, J. A. Absence of Fyn and Src causes a reeler-like phenotype. The Journal of neuroscience : the official journal of the Society for Neuroscience 25, 8578-8586, doi:10.1523/JNEUROSCI.1656-05.2005 (2005).

28 Lowe, C. et al. Osteopetrosis in Src-deficient mice is due to an autonomous defect of osteoclasts. Proceedings of the National Academy of Sciences of the United States of America 90, 4485-4489 (1993).

29 Lowell, C. A. \& Soriano, P. Knockouts of Src-family kinases: stiff bones, wimpy T cells, and bad memories. Genes \& development 10, 1845-1857 (1996).

30 Marchetti, D., Parikh, N., Sudol, M. \& Gallick, G. E. Stimulation of the protein tyrosine kinase c-Yes but not c-Src by neurotrophins in human brain-metastatic melanoma cells. Oncogene 16, 3253-3260, doi:10.1038/sj.onc.1201877 (1998).

31 Molina, T. J. et al. Profound block in thymocyte development in mice lacking p56lck. Nature 357, 161-164, doi:10.1038/357161a0 (1992).

32 Palacios-Moreno, J. et al. Neuroblastoma tyrosine kinase signaling networks involve FYN and LYN in endosomes and lipid rafts. PLoS computational biology 11, e1004130, doi:10.1371/journal.pcbi.1004130 (2015). 
33 Paster, W. et al. Genetically encoded Forster resonance energy transfer sensors for the conformation of the Src family kinase Lck. Journal of immunology 182, 2160-2167, doi:10.4049/jimmunol.0802639 (2009).

34 Koudelkova, L. et al. Novel FRET-Based Src Biosensor Reveals Mechanisms of Src Activation and Its Dynamics in Focal Adhesions. Cell chemical biology 26, 255-268 e254, doi:10.1016/j.chembiol.2018.10.024 (2019).

35 Du, C. et al. KLF5 promotes cell migration by up-regulating FYN in bladder cancer cells. FEBS letters 590, 408-418, doi:10.1002/1873-3468.12069 (2016).

36 Lewin, B. et al. Expression of Fyn kinase modulates EMT in oral cancer cells. Anticancer research 30, 2591-2596 (2010).

37 Meriane, M. et al. Phosphorylation of DCC by Fyn mediates Netrin-1 signaling in growth cone guidance. The Journal of cell biology 167, 687-698, doi:10.1083/jcb.200405053 (2004).

38 Posadas, E. M. et al. Saracatinib as a metastasis inhibitor in metastatic castrationresistant prostate cancer: A University of Chicago Phase 2 Consortium and DOD/PCF Prostate Cancer Clinical Trials Consortium Study. The Prostate 76, 286-293, doi:10.1002/pros.23119 (2016).

39 Salter, M. W. \& Kalia, L. V. Src kinases: a hub for NMDA receptor regulation. Nature reviews. Neuroscience 5, 317-328, doi:10.1038/nrn1368 (2004).

40 Martin-Avila, A. et al. Protein Tyrosine Kinase Fyn Regulates TLR4-Elicited Responses on Mast Cells Controlling the Function of a PP2A-PKCalpha/beta Signaling Node Leading to TNF Secretion. Journal of immunology 196, 50755088, doi:10.4049/jimmunol.1501823 (2016).

41 Yadav, V. \& Denning, M. F. Fyn is induced by Ras/PI3K/Akt signaling and is required for enhanced invasion/migration. Molecular carcinogenesis 50, 346352, doi:10.1002/mc.20716 (2011).

42 Arold, S. T. et al. The role of the Src homology 3-Src homology 2 interface in the regulation of Src kinases. The Journal of biological chemistry 276, 17199-17205, doi:10.1074/jbc.M011185200 (2001).

43 Boggon, T. J. \& Eck, M. J. Structure and regulation of Src family kinases. Oncogene 23, 7918-7927, doi:10.1038/sj.onc.1208081 (2004).

44 Kinoshita, T., Matsubara, M., Ishiguro, H., Okita, K. \& Tada, T. Structure of human Fyn kinase domain complexed with staurosporine. Biochemical and biophysical research communications 346, 840-844, doi:10.1016/j.bbrc.2006.05.212 (2006).

45 Noble, M. E., Musacchio, A., Saraste, M., Courtneidge, S. A. \& Wierenga, R. K. Crystal structure of the SH3 domain in human Fyn; comparison of the threedimensional structures of SH3 domains in tyrosine kinases and spectrin. The EMBO journal 12, 2617-2624 (1993).

46 Sicheri, F. \& Kuriyan, J. Structures of Src-family tyrosine kinases. Current opinion in structural biology 7, 777-785 (1997).

47 Young, M. A., Gonfloni, S., Superti-Furga, G., Roux, B. \& Kuriyan, J. Dynamic coupling between the $\mathrm{SH} 2$ and $\mathrm{SH} 3$ domains of c-Src and Hck underlies their inactivation by C-terminal tyrosine phosphorylation. Cell 105, 115-126 (2001).

48 Gera, N., Hill, A. B., White, D. P., Carbonell, R. G. \& Rao, B. M. Design of pH sensitive binding proteins from the hyperthermophilic Sso7d scaffold. PloS one 7, e48928, doi:10.1371/journal.pone.0048928 (2012). 
49 Gera, N., Hussain, M., Wright, R. C. \& Rao, B. M. Highly stable binding proteins derived from the hyperthermophilic Sso7d scaffold. Journal of molecular biology 409, 601-616, doi:10.1016/j.jmb.2011.04.020 (2011).

50 Gocha, T., Rao, B. M. \& DasGupta, R. Identification and characterization of a novel Sso7d scaffold-based binder against Notch1. Scientific reports 7, 12021, doi:10.1038/s41598-017-12246-1 (2017).

51 Hussain, M., Lockney, D., Wang, R., Gera, N. \& Rao, B. M. Avidity-mediated virus separation using a hyperthermophilic affinity ligand. Biotechnology progress 29, 237-246, doi:10.1002/btpr.1655 (2013).

52 Gera, N., Hussain, M. \& Rao, B. M. Protein selection using yeast surface display. Methods 60, 15-26, doi:10.1016/j.ymeth.2012.03.014 (2013).

53 van der Schot, G. et al. Improving 3D structure prediction from chemical shift data. Journal of biomolecular NMR 57, 27-35, doi:10.1007/s10858-013-9762-6 (2013).

54 de Vries, S. J., van Dijk, M. \& Bonvin, A. M. The HADDOCK web server for data-driven biomolecular docking. Nature protocols 5, 883-897, doi:10.1038/nprot.2010.32 (2010).

55 van Zundert, G. C. P. et al. The HADDOCK2.2 Web Server: User-Friendly Integrative Modeling of Biomolecular Complexes. Journal of molecular biology 428, 720-725, doi:10.1016/j.jmb.2015.09.014 (2016).

56 Li, S. S. Specificity and versatility of SH3 and other proline-recognition domains: structural basis and implications for cellular signal transduction. The Biochemical journal 390, 641-653, doi:10.1042/BJ20050411 (2005).

57 Takeuchi, M. et al. Functional and physical interaction of protein-tyrosine kinases Fyn and Csk in the T-cell signaling system. The Journal of biological chemistry 268, 27413-27419 (1993).

58 Trinh, R., Gurbaxani, B., Morrison, S. L. \& Seyfzadeh, M. Optimization of codon pair use within the (GGGGS)3 linker sequence results in enhanced protein expression. Molecular immunology 40, 717-722 (2004).

59 Cooper, J. A. \& MacAuley, A. Potential positive and negative autoregulation of p60c-src by intermolecular autophosphorylation. Proceedings of the National Academy of Sciences of the United States of America 85, 4232-4236 (1988).

60 Barker, S. C. et al. Characterization of pp60c-src tyrosine kinase activities using a continuous assay: autoactivation of the enzyme is an intermolecular autophosphorylation process. Biochemistry 34, 14843-14851 (1995).

61 Wary, K. K., Mariotti, A., Zurzolo, C. \& Giancotti, F. G. A requirement for caveolin-1 and associated kinase Fyn in integrin signaling and anchoragedependent cell growth. Cell 94, 625-634 (1998).

62 van't Hof, W. \& Resh, M. D. Dual fatty acylation of p59(Fyn) is required for association with the $\mathrm{T}$ cell receptor zeta chain through phosphotyrosine-Src homology domain-2 interactions. The Journal of cell biology 145, 377-389 (1999).

63 Victor, K. \& Cafiso, D. S. Structure and position of the N-terminal membranebinding domain of pp60src at the membrane interface. Biochemistry 37, 34023410, doi:10.1021/bi9721501 (1998).

64 Etienne-Manneville, S. \& Hall, A. Integrin-mediated activation of Cdc42 controls cell polarity in migrating astrocytes through PKCzeta. Cell 106, 489-498 (2001). 
65 Huveneers, S. \& Danen, E. H. Adhesion signaling - crosstalk between integrins, Src and Rho. Journal of cell science 122, 1059-1069, doi:10.1242/jcs.039446 (2009).

66 Roca-Cusachs, P., Iskratsch, T. \& Sheetz, M. P. Finding the weakest link: exploring integrin-mediated mechanical molecular pathways. Journal of cell science 125, 3025-3038, doi:10.1242/jcs.095794 (2012).

67 Hodgson, L. et al. FRET binding antenna reports spatiotemporal dynamics of GDI-Cdc42 GTPase interactions. Nature chemical biology 12, 802-809, doi:10.1038/nchembio.2145 (2016).

68 Karpova, T. \& McNally, J. G. Detecting protein-protein interactions with CFPYFP FRET by acceptor photobleaching. Current protocols in cytometry Chapter 12, Unit12 17, doi:10.1002/0471142956.cy1207s35 (2006).

69 Blake, R. A. et al. SU6656, a selective src family kinase inhibitor, used to probe growth factor signaling. Molecular and cellular biology 20, 9018-9027 (2000).

70 Krishnamurty, R. et al. Active site profiling reveals coupling between domains in SRC-family kinases. Nature chemical biology 9, 43-50, doi:10.1038/nchembio.1118 (2013).

71 Gordon, G. W., Berry, G., Liang, X. H., Levine, B. \& Herman, B. Quantitative fluorescence resonance energy transfer measurements using fluorescence microscopy. Biophysical journal 74, 2702-2713, doi:10.1016/S00063495(98)77976-7 (1998).

72 Mills, R. D. et al. A role for the tyrosine kinase Pyk2 in depolarization-induced contraction of vascular smooth muscle. The Journal of biological chemistry $\mathbf{2 9 0}$, 8677-8692, doi:10.1074/jbc.M114.633107 (2015).

73 Stokes, J. B. et al. Inhibition of focal adhesion kinase by PF-562,271 inhibits the growth and metastasis of pancreatic cancer concomitant with altering the tumor microenvironment. Molecular cancer therapeutics 10, 2135-2145, doi:10.1158/1535-7163.MCT-11-0261 (2011).

74 Renshaw, M. W., Price, L. S. \& Schwartz, M. A. Focal adhesion kinase mediates the integrin signaling requirement for growth factor activation of MAP kinase. The Journal of cell biology 147, 611-618 (1999).

75 Cheng, S. Y., Sun, G., Schlaepfer, D. D. \& Pallen, C. J. Grb2 promotes integrininduced focal adhesion kinase (FAK) autophosphorylation and directs the phosphorylation of protein tyrosine phosphatase alpha by the Src-FAK kinase complex. Molecular and cellular biology 34, 348-361, doi:10.1128/MCB.0082513 (2014).

76 Coster, A. D., Thorne, C. A., Wu, L. F. \& Altschuler, S. J. Examining Crosstalk among Transforming Growth Factor beta, Bone Morphogenetic Protein, and Wnt Pathways. The Journal of biological chemistry 292, 244-250, doi:10.1074/jbc.M116.759654 (2017).

77 Warmflash, A. et al. Dynamics of TGF-beta signaling reveal adaptive and pulsatile behaviors reflected in the nuclear localization of transcription factor Smad4. Proceedings of the National Academy of Sciences of the United States of America 109, E1947-1956, doi:10.1073/pnas.1207607109 (2012).

78 Weber, T. J. et al. Basic fibroblast growth factor regulates persistent ERK oscillations in premalignant but not malignant JB6 cells. The Journal of investigative dermatology 130, 1444-1456, doi:10.1038/jid.2009.383 (2010). 
79 Avraham, R. \& Yarden, Y. Feedback regulation of EGFR signalling: decision making by early and delayed loops. Nature reviews. Molecular cell biology 12, 104-117, doi:10.1038/nrm3048 (2011).

80 Sparta, B. et al. Receptor Level Mechanisms Are Required for Epidermal Growth Factor (EGF)-stimulated Extracellular Signal-regulated Kinase (ERK) Activity Pulses. The Journal of biological chemistry 290, 24784-24792, doi:10.1074/jbc.M115.662247 (2015).

81 Albeck, J. G., Mills, G. B. \& Brugge, J. S. Frequency-modulated pulses of ERK activity transmit quantitative proliferation signals. Molecular cell 49, 249-261, doi:10.1016/j.molcel.2012.11.002 (2013).

82 DeRita, R. M. et al. c-Src, Insulin-Like Growth Factor I Receptor, G-ProteinCoupled Receptor Kinases and Focal Adhesion Kinase are Enriched Into Prostate Cancer Cell Exosomes. J Cell Biochem 118, 66-73, doi:10.1002/jcb.25611 (2017).

83 Edick, M. J., Tesfay, L., Lamb, L. E., Knudsen, B. S. \& Miranti, C. K. Inhibition of integrin-mediated crosstalk with epidermal growth factor receptor/Erk or Src signaling pathways in autophagic prostate epithelial cells induces caspaseindependent death. Molecular biology of the cell 18, 2481-2490, doi:10.1091/mbc.E06-04-0261 (2007).

84 Samayawardhena, L. A., Kapur, R. \& Craig, A. W. Involvement of Fyn kinase in Kit and integrin-mediated Rac activation, cytoskeletal reorganization, and chemotaxis of mast cells. Blood 109, 3679-3686, doi:10.1182/blood-2006-11057315 (2007).

85 Parsons, J. T. \& Parsons, S. J. Src family protein tyrosine kinases: cooperating with growth factor and adhesion signaling pathways. Current opinion in cell biology 9, 187-192 (1997).

86 Lehembre, F. et al. NCAM-induced focal adhesion assembly: a functional switch upon loss of E-cadherin. The EMBO journal 27, 2603-2615, doi:10.1038/emboj.2008.178 (2008).

87 Kinnunen, T. et al. Cortactin-Src kinase signaling pathway is involved in Nsyndecan-dependent neurite outgrowth. The Journal of biological chemistry 273, 10702-10708 (1998).

88 Arias-Salgado, E. G., Lizano, S., Shattil, S. J. \& Ginsberg, M. H. Specification of the direction of adhesive signaling by the integrin beta cytoplasmic domain. The Journal of biological chemistry 280, 29699-29707, doi:10.1074/jbc.M503508200 (2005).

89 Bhaskar, K., Yen, S. H. \& Lee, G. Disease-related modifications in tau affect the interaction between Fyn and Tau. The Journal of biological chemistry 280, 35119-35125, doi:10.1074/jbc.M505895200 (2005).

90 Schenone, S. et al. Fyn kinase in brain diseases and cancer: the search for inhibitors. Current medicinal chemistry 18, 2921-2942 (2011).

91 Chin, J. et al. Fyn kinase induces synaptic and cognitive impairments in a transgenic mouse model of Alzheimer's disease. The Journal of neuroscience : the official journal of the Society for Neuroscience 25, 9694-9703, doi:10.1523/JNEUROSCI.2980-05.2005 (2005).

92 Colognato, H. et al. CNS integrins switch growth factor signalling to promote target-dependent survival. Nature cell biology 4, 833-841, doi:10.1038/ncb865 (2002). 
93 Colognato, H., Ramachandrappa, S., Olsen, I. M. \& ffrench-Constant, C. Integrins direct Src family kinases to regulate distinct phases of oligodendrocyte development. The Journal of cell biology 167, 365-375, doi:10.1083/jcb.200404076 (2004).

94 Laursen, L. S., Chan, C. W. \& ffrench-Constant, C. An integrin-contactin complex regulates CNS myelination by differential Fyn phosphorylation. The Journal of neuroscience : the official journal of the Society for Neuroscience $\mathbf{2 9}$, 9174-9185, doi:10.1523/JNEUROSCI.5942-08.2009 (2009).

95 Laursen, L. S., Chan, C. W. \& Ffrench-Constant, C. Translation of myelin basic protein mRNA in oligodendrocytes is regulated by integrin activation and hnRNP-K. The Journal of cell biology 192, 797-811, doi:10.1083/jcb.201007014 (2011).

96 Laursen, L. S. \& Ffrench-Constant, C. Adhesion molecules in the regulation of CNS myelination. Neuron glia biology 3, 367-375, doi:10.1017/S1740925X08000161 (2007).

97 Sperber, B. R. \& McMorris, F. A. Fyn tyrosine kinase regulates oligodendroglial cell development but is not required for morphological differentiation of oligodendrocytes. Journal of neuroscience research 63, 303-312, doi:10.1002/1097-4547(20010215)63:4<303::AID-JNR1024>3.0.CO;2-A (2001).

98 Schafer, I., Muller, C., Luhmann, H. J. \& White, R. MOBP levels are regulated by Fyn kinase and affect the morphological differentiation of oligodendrocytes. Journal of cell science 129, 930-942, doi:10.1242/jcs.172148 (2016).

99 Imayoshi, I. et al. Oscillatory control of factors determining multipotency and fate in mouse neural progenitors. Science 342, 1203-1208, doi:10.1126/science.1242366 (2013).

100 Isomura, A. \& Kageyama, R. Ultradian oscillations and pulses: coordinating cellular responses and cell fate decisions. Development 141, 3627-3636, doi:10.1242/dev.104497 (2014).

101 Antoine-Bertrand, J., Fu, M. \& Lamarche-Vane, N. Direct measurement of oscillatory RhoA activity in embryonic cortical neurons stimulated with the axon guidance cue netrin-1 using fluorescence resonance energy transfer. Biology of the cell 108, 115-126, doi:10.1111/boc.201500077 (2016).

102 Shankaran, H. et al. Rapid and sustained nuclear-cytoplasmic ERK oscillations induced by epidermal growth factor. Molecular systems biology 5, 332, doi:10.1038/msb.2009.90 (2009).

103 Delaglio, F. et al. NMRPipe: a multidimensional spectral processing system based on UNIX pipes. Journal of biomolecular NMR 6, 277-293 (1995).

104 Kneller, D. G. \& Kuntz, I. D. Ucsf Sparky - an Nmr Display, Annotation and Assignment Tool. J Cell Biochem, 254-254 (1993).

105 Lee, W., Westler, W. M., Bahrami, A., Eghbalnia, H. R. \& Markley, J. L. PINESPARKY: graphical interface for evaluating automated probabilistic peak assignments in protein NMR spectroscopy. Bioinformatics 25, 2085-2087, doi:10.1093/bioinformatics/btp345 (2009).

106 Lange, O. F. et al. Determination of solution structures of proteins up to $40 \mathrm{kDa}$ using CS-Rosetta with sparse NMR data from deuterated samples. Proceedings of the National Academy of Sciences of the United States of America 109, 1087310878, doi:10.1073/pnas.1203013109 (2012). 
107 Kunkel, T. A., Roberts, J. D. \& Zakour, R. A. Rapid and efficient site-specific mutagenesis without phenotypic selection. Methods in enzymology 154, 367-382 (1987).

108 Zheng, L., Baumann, U. \& Reymond, J. L. An efficient one-step site-directed and site-saturation mutagenesis protocol. Nucleic acids research 32, e115, doi:10.1093/nar/gnh110 (2004).

109 Bryksin, A. V. \& Matsumura, I. Overlap extension PCR cloning: a simple and reliable way to create recombinant plasmids. BioTechniques 48, 463-465, doi:10.2144/000113418 (2010).

110 Canny, J. A computational approach to edge detection. IEEE transactions on pattern analysis and machine intelligence 8, 679-698 (1986).

111 Mathworks. Find edges in intensity image, <https://www.mathworks.com/help/images/ref/edge.html\#d120e54923> (2019).

112 Karpova, T. S. et al. Fluorescence resonance energy transfer from cyan to yellow fluorescent protein detected by acceptor photobleaching using confocal microscopy and a single laser. Journal of microscopy 209, 56-70 (2003).

113 Lim, J. S. Two-dimensional signal and image processing. Vol. XVI, 694p 469476 (Englewood Cliffs, N.J. : Prentice Hall, 1990).

114 Hachet-Haas, M. et al. FRET and colocalization analyzer--a method to validate measurements of sensitized emission FRET acquired by confocal microscopy and available as an ImageJ Plug-in. Microscopy research and technique 69, 941-956, doi:10.1002/jemt.20376 (2006).

115 Oldach, L. M., Gorshkov, K., Mills, W. T. t., Zhang, J. \& Meffert, M. K. A biosensor for MAPK-dependent Lin28 signaling. Molecular biology of the cell 29, 1157-1167, doi:10.1091/mbc.E17-08-0500 (2018).

116 Proakis John G., M. D. G. Digital Signal Processing: Principles, Algorithms, and Applications. Third edn, (Prentice-Hall International, 1996).

117 Mitov, I. P. A method for assessment and processing of biomedical signals containing trend and periodic components. Medical engineering \& physics $\mathbf{2 0}$, 660-668 (1998).

118 Colak, O. H. Preprocessing effects in time-frequency distributions and spectral analysis of heart rate variability. Digital Signal Processing 19, 731-739, doi:https://doi.org/10.1016/j.dsp.2008.09.004 (2009).

119 Mathworks. Find Periodicity Using Frequency Analysis, $<$ https://in.mathworks.com/help/signal/ug/find-periodicity-using-frequencyanalysis.html > (2019). 


\title{
SUPPLEMENTARY INFORMATION
}

\author{
A Fyn - specific biosensor reveals localized, pulsatile kinase activity and spatially \\ regulated signaling crosstalk
}

Ananya Mukherjee*,1,4, Randhir Singh*,1, Sreeram Udayan ${ }^{1}$, Sayan Biswas ${ }^{1}$, Purushotham Pothula $^{2}$, Saumya Manmadhan ${ }^{1}$, Geen ${ }^{1}$, Shilpa Dilip Kumar ${ }^{1}$, Ranabir Das ${ }^{2}$, Balaji M.Rao ${ }^{3 凶}$, and Akash Gulyani ${ }^{1 凶}$

\section{Authors Affiliations}

1. Institute for Stem Cell Science and Regenerative Medicine, Bangalore, India

2. National Centre for Biological Sciences, Bangalore, India

3. North Carolina State University, Raleigh, North Carolina, USA

4. SASTRA University, Thanjavur, Tamil Nadu, India

*; Equal contribution

To whom correspondence should be addressed:

Email: akashg@instem.res.in

bmrao@ncsu.edu

Phone: +918061948230 


\section{Chemical and reagents}

I. Sigma-Aldrich- IPTG, EDTA, Glutathione, BSA, Manganese(II) chloride, KCl, Bromophenol blue, Potassium acetate, $\mathrm{D}(+)$ Galatose, $\mathrm{CaCl} 2, \mathrm{D}(+)$ Glucose, $p$ Coumaric acid, Luminol, DMSO, Imidazole, Biotinamidohexanoic acid Nhydroxysuccinimide-ester, Ampicillin Sodium salt, Penicillin-Streptomycin, Yeast Nitrogen base without amino acids, Fibronectin solution, phenylmethylsulfonyl fluoride, Platelet-derived Growth Factor, Ethidium Bromide, puromycinand Tween20 and Dialysis tubing.

II. Fisher Scientific- Disodium Phosphates, Sodium Dihydrogen Phosphate, Tris-buffer.

III. HiMedia Biosciences-NaCl, SDS, McCoy's 5A media, Luria Broth and Agar, FBS.

IV. Merck- Anhydrous Glycerol, Glacial Acetic acid, Hydrochloric acid and Isopropanol

V. Thermo-Fisher Scientific- Dynabeads Biotin Binder (\#11047), PVDF-membrane, Lipofectamine LTX and plus reagent, Lipofectamine 3000, RIPA Lysis buffer, Pierce Phosphatase Inhibitor tablets, Pierce BCA assay kit, Page-Ruler Plus prestained protein ladder (10 to $250 \mathrm{kDa}$ ), Ni-NTA agarose beads, Agarose, DMEM (high glucose), DMEM (high glucose, HEPES, -phenol red), 1X DPBS, GlutaMax.

VI. Bio-RAD- 30\% acrylamide/ Bis solution, Stacking and resolving gel buffer, TAE electrophoresis buffer and Econo-chromatography Columns.

VII. GE healthcare- Glutathione sepharose 4 Fast Flow and Superdex-75GL Size Exclusion Chromatography Column.

VIII. Zymoresearch- Zymoprep yeast plasmid miniprep II kit.

IX. Promega- Pure-yield plasmid miniprep and wizard SV gelPCR clean-up system.

X. Roche- Protease inhibitor cocktail tablets, APS.

XI. $\quad \boldsymbol{N E B}$-Phusion high-fidelity DNA Polymerase, Q5 high fidelity DNA polymerases, T4 DNA ligase, DpnI, NcoI-HF, BamHI-HF, EcoRI-HF, alkaline phosphatise (CIP), $1 \mathrm{~kb}$ \& 100 bp DNA Ladder.

XII. Clontech-TaKaRa Ex-Taq, LA-Taq r-Taq DNA Polymerase.

XIII. EMD-Millipore- SU6656 (572635-1MG).

XIV. Selleckchem-FAK inhibitor PF-562271 (Catalog No. S2890).

XV. Santa Cruz Biotechnology-Polybrene (sc-134220).

\section{Antibodies}

I. Invitrogen-c-Myc chicken IgY antibody; goat anti-chicken $\operatorname{IgY}(\mathrm{H}+\mathrm{L})$ secondary antibody; Alexa Fluor 633 conjugate; Avidin-Neutravidin FITC conjugate and Streptavidin R-phycoerythrin conjugate (SAPE). Goat anti-mouse IgG secondary antibody-Alexa Fluor 647 conjugate; and Donkey anti-rabbit secondary antibody-Alexa Fluor 546 conjugate.

II. Cell Signaling Technology- phospho-Src family (Tyr416) antibody; phospho-Src (Tyr527) antibody; total Fyn antibody; $\beta$-actin antibody; p44/42 MAPK (Erk1/2) antibody, Phospho-p44/42 MAPK (Erk1/2) (Thr202/Tyr204) antibody, Anti-mouse IgG-HRP-linked antibody and Anti-rabbit IgG- HRP-linked antibody.

III. Abcam- anti-GFP antibody, Anti-Fyn Mouse monoclonal antibody (IF grade)

IV. Sigma-monoclonal anti-polyhistidine antibody, monoclonal anti-vinculin antibody, anti-Src polyclonal antibody produced in rabbit, anti-yes polyclonal antibody produced in rabbit. 
bioRxiv preprint doi: https://doi.org/10.1101/726133; this version posted August 5, 2019. The copyright holder for this preprint (which was not certified by peer review) is the author/funder, who has granted bioRxiv a license to display the preprint in perpetuity. It is made available under aCC-BY-NC-ND 4.0 International license.

\section{Primers procurement and DNA sequencing}

Primers used in study were procured from Bioserve Biotechnologies (India). The oligonucleotide synthesis scale was $25 \mathrm{nmol}$. Primers up to 50 nucleotide lengths were desalted, however, longer primers were PAGE purified. All DNA sequencing was performed on Illumina- MiSeq DNA Sequencer machine at the DNA sequencing facility at NCBS.

\section{Purification of SH3 domains}

Domains of individual Src kinase were encoded in a pET-14b plasmid, so that a ' $6 \mathrm{X}$-HisSUMO-Thr-SH3-domain' fusion protein could be expressed. The plasmid was transformed into competent $E$. coli BL21-(DE3) cells using heat shock method. The late-log phase grown E. coli cells (OD $\sim 0.8$ ) were induced with $0.5 \mathrm{mM}$ of IPTG for $3 \mathrm{hrs}$ at $37^{\circ} \mathrm{C}$, at $200 \mathrm{rpm}$ in a shaker incubator. Cells were harvested and washed once with $1 \mathrm{X}$ PBS buffer by centrifugation at $8,000 \times \mathrm{g}$ and then re-suspended 1:4 wt./vol in ice-cold Buffer-A (50 mM sodium phosphate buffer, $500 \mathrm{mM} \mathrm{NaCl}, \mathrm{pH} 7.5$ ) having $10 \mathrm{mM}$ imidazole followed by sonication with an ultrasonic processor on ice, with a total of 4 cycles consisting of 3 min cycle of $4 \mathrm{~s}$ ON time and $10 \mathrm{~s}$ OFF time at $40 \%$ amplitude. The cell-homogenate was centrifuged at $35,000 \times \mathrm{g}$ for $35 \mathrm{~min}$. The clear supernatant cell-free extract (CFE) was incubated for $4 \mathrm{~h}$ with Ni-NTA beads (Invitrogen) at $4^{\circ} \mathrm{C}$ under mild rotation condition. The CFE with beads were packed on to glass econo-columns (Bio-Rad). The unbound protein was washed twice with Buffer-A having $10 \mathrm{mM}$ imidazole and elutions were carried out using a step gradient of Buffer-A containing imidazole $(50 \mathrm{mM}$ to $250 \mathrm{mM}, 2 \mathrm{ml}$ each). Imidazole was removed by dialysis against BufferA. Protein fractions were further purified using Superdex 75GL size exclusion chromatography column (AKTA pure, GE healthcare life science) with Buffer-A. The protein preps were analysed using $12 \%$ SDS-PAGE for their purity and homogeneity. The fractions having protein of interest were pooled and concentrated using Amicon ultra-centrifugal filter $10 \mathrm{~K}$ (Millipore).

\section{Binder Screening Steps}

Stable Sso7d protein of Sulfolobus solfataricus was used as scaffold in binder screening. Earlier, a library of $\sim 10^{8} \mathrm{Sso} 7 \mathrm{~d}$ mutants had been generated by randomizing 10 amino acid residues on the DNA-binding surface of Sso7d, using yeast surface display platform ${ }^{1}$. The Sso7d scaffold library in the yeast surface display platform has been used to isolate the specific binder for SH3 domain of SFK, Fyn. The screening procedures were followed as described earlier ${ }^{1}$ and are explained below and in Supplementary Fig. 1.

i. Target biotinylation-SH3-domains of SFKs were biotinylated using Biotinamidohexanoic acid N-hydroxysuccinimide-ester followed by purification using SephadexG-10 column.

ii. Target coating on magnetic beads- Biotinylated SH3 domains $(1 \mu \mathrm{M})$ were pre-incubated with $2.5 \times 10^{6}$ dynabeads biotin binder in $1 \mathrm{X}$ PBS $(100 \mu 1$ system $)$ at $4^{\circ} \mathrm{C}$ at $5 \mathrm{rpm}$ on $360^{\circ}$ rotor. Target preloaded beads were washed twice with PBS-BSA (0.1\% BSA in $1 \mathrm{X}$ PBS) and captured using the Dyna-Mag2 magnet.

iii. Expansion and induction of yeast cell library - $2 \times 10^{8}$ cells were cultured from frozen stock of yeast cell library of Sso7d in 1L of SDCAA media with pen-strep (1:100) and incubated at $30^{\circ} \mathrm{C}, 200 \mathrm{rpm}$ shaker for $72 \mathrm{~h}$. From the expanded culture, $\sim 10^{9}$ cells were inoculated into $50 \mathrm{ml}$ of SGCAA media having pen-strep for $20 \mathrm{~h}$ at $30^{\circ} \mathrm{C}$ in order to induce the expression of mutant proteins on the yeast cell surface.

iv. Magnetic screening- $\sim 10^{9}$ cells from the induced culture (step iii) were centrifuged at 2500 $\mathrm{x} g$ for $3 \mathrm{~min}$ and the pellet dissolved in $1 \mathrm{ml}$ of PBS-BSA. Two rounds of negative selection against beads were carried out by incubating cells with the washed beads for $1 \mathrm{~h}$ at $4^{\circ} \mathrm{C}$ in a 
microcentrifuge tube, in a rotator followed by unbound yeast cell collection using magnet. The unbound yeast cells were incubated in a stepwise manner with $1 \mu \mathrm{M}$ biotinylated target (SH3 domains other than Fyn) in microcentrifuge tube for $1 \mathrm{~h}$ at $4^{\circ} \mathrm{C}$ in a rotator followed by unbound yeast cell collection using magnet in order to deselect binder. The probable Fyn SH3 binders were selected from previous unbound yeast cells by incubating with $1 \mu \mathrm{M}$ biotinylated target (Fyn SH3 domain) in a microcentrifuge tube for $1 \mathrm{~h}$ at $4^{\circ} \mathrm{C}$ in a rotator. The bead-yeast complex was isolated, washed twice with 1X PBS and transferred to a culture tube containing $5 \mathrm{ml}$ SDCAA with pen-strep and incubated for $48 \mathrm{~h}$ in a shaking condition at $30^{\circ} \mathrm{C}$, at $200 \mathrm{rpm}$. The beads were removed using the magnet and the yeast cells were further inoculated in a $50 \mathrm{ml}$ SDCAA culture with pen-strep for $48 \mathrm{~h}$ in a shaking condition at $30^{\circ} \mathrm{C}$, at $200 \mathrm{rpm}$.

v. Screening using FACS-The yeast cells obtained after magnetic screening were further screened using flow cytometry to isolate the highest affinity Fyn binders. In this step, inorder to isolate yeast cells bound to the target SH3 domain, two fluorescent signals were used. Target SH3 domain presence was detected by streptavidin, R-Phycoerythrin Conjugate (SAPE, against biotinylated SH3 domain). The yeast cells expressing full-length scaffold were detected by Alexa-Fluor 633 labeled secondary antibody against c-myc. Presence of dual staining in FACS experiments highlights events where yeast cells with scaffold bind the target (SH3 domain) and has been used for screening for binding and validation ${ }^{1}$. The Fyn binder was isolated after six subsequent stringent sorting steps using Fyn SH3 domains as a target. Finally, clean population in FACS were gated and sorted. The specificity of the pool was determined by FACS against SFKs SH3 domain (Supplementary Fig. 1). Yeast cells from this pool were plated onto SDCAA agar plates and incubated at $30^{\circ} \mathrm{C}$ for $48 \mathrm{~h}$. More than 30 randomly picked colonies were inoculated in $5 \mathrm{ml}$ SDCAA media and incubated for $48 \mathrm{~h}$ at $30^{\circ} \mathrm{C}$, at $200 \mathrm{rpm}$ in a shaker incubator. Specificity of each clone was tested against other SFK SH3 domains $(1 \mu \mathrm{M})$. We have selected a yeast clone number 29 for its specificity against Fyn SH3 domain compared to others using FACS (Fig. 1c, Supplementary Fig. 2). Plasmid was isolated from the yeast clone no. 29 using the Zymoprep kit (Zymo-Corp USA) and the DNA was sequenced. This clone is hereafter referred to as 'F29' (Fyn binder 29).

\section{Pull-down of SH3 domain with binders}

Pull-down experiments were performed to validate the interaction of binder F29 and Fyn SH3 domain. In the plasmid p-GST-ppx-L $\mathrm{L}_{15}-\mathrm{F} 29$, we have made P41A and R33A mutations in F29 ORF using site directed mutagenesis to yield plasmid p-GST-ppx-L15-F29-P41A \& p-GSTppx-L $15-F 29-R 331$ with primers SN 10,11 \& 12,13 respectively (Supplementary Table 1 \& 2).

i. Bead immobilization with bait protein and Pulldown-The purified GST fused binder (GSTF29) or its point mutant (GST-F29-P41A and GST-F29-R33A) were incubated at room temperature for 2 hours with glutathione sepharose beads (i.e. $10 \mu l$ beads with $100 \mu \mathrm{g}$ of protein) under mild $360^{\circ}$ rotating conditions $(5 \mathrm{rpm})$. Incubated beads were washed four times with $1 \mathrm{X}$-PBS buffer to remove the unbound, excess protein. From washed pool of GST-F29 saturated beads, $\sim 10 \mu \mathrm{l}$ beads was incubated with varying concentrations of $6 \mathrm{X}-$ His-Fyn-SH3 domains $(75 \mathrm{nM}$ to $1.25 \mu \mathrm{M})$ ina $100 \mu \mathrm{l}$ of reaction system for $4 \mathrm{~h}$ at $4{ }^{\circ} \mathrm{C}$ under mild rotating $360^{\circ}$ rotations. Each tube was washed four times with $1 \mathrm{ml}$ ice cold $1 \mathrm{X}$-PBS to remove unbound, excess SH3 domain. The beads were then boiled with $30 \mu 1$ of $1 \mathrm{X}$ Laemmli buffer for $10 \mathrm{~min}$ at $98^{\circ} \mathrm{C}$. Using these samples, we have performed SDS gel $(12 \%)$ electrophoresis for immunoblot assay. In order to show that GST protein does not interact with $6 \mathrm{X}$-His- Fyn SH3 domain, we have performed apulldown experiments with $10 \mu \mathrm{l}$ 
bioRxiv preprint doi: https://doi.org/10.1101/726133; this version posted August 5, 2019. The copyright holder for this preprint (which was not certified by peer review) is the author/funder, who has granted bioRxiv a license to display the preprint in perpetuity. It is made available under aCC-BY-NC-ND 4.0 International license.

glutathione sepharose beads saturated with $100 \mu \mathrm{g}$ of GST protein and incubated with $1000 \mathrm{nM} 6 \mathrm{X}$-His-Fyn-SH3 domain in a $100 \mu \mathrm{l}$ of reaction system using conditions similar to above. Similarly,500, 750 and 1000nM of $6 \mathrm{X}$-His-Fyn-SH3 was pulled-down using $10 \mu 1$ beads saturated with GST-F29protein and was run on a $12 \%$ SDS-gel. The gel region spanning 29 to $43 \mathrm{kDa}$ region was stained with Coomassie blue dye, and 29 to 16kDa region was use in immunoblot assay as described below.

ii. Immunoblot assay-The proteins from SDS gel were transferred onto PVDF membrane (Invitrogen, $0.2 \mu \mathrm{m}$ ) at $100 \mathrm{~V}$ for $90 \mathrm{~min}$ at $4^{\circ} \mathrm{C}$ in $1 \mathrm{X}$ transfer Buffer $(25 \mathrm{mM}$ Tris- $\mathrm{HCl} ; 190$ $\mathrm{mM}$ glycine and $20 \%$ methanol). After complete transfer the PVDF membrane was incubated (facing up) in blocking buffer (5\% non-fat dry milk, Blotto) in 1X-TBST (20mM Tris-Cl pH 7.6, $150 \mathrm{mM} \mathrm{NaCl}, \mathrm{pH} \mathrm{7.6}$, and $0.1 \%$ Tween 20) for $1 \mathrm{hr}$ at room temperature on a shaker. The blot was then incubated in hybridization bags with a primary antibody (anti-polyhistidine antibody, 1:3000) in antibody dilution buffer (5\% BSA in 1X-TBST) at $4^{\circ} \mathrm{C}$, overnight under mild shaking conditions. The membrane was washed stringently with 1X-TBST and incubated with anti-mouse IgG, HRP-linked secondary antibody (1:3000) at room temperature for $1 \mathrm{hr}$. The membrane was washed 4 times with 1X-TBST and imaged using Image-Quant LAS 4000 (GE-Healthcare) in luminol-based enhanced chemiluminescence reagent. The band intensity of blot was analysed using Image-J(NIH). Similar experiments were performed with Src SH3 domain and its mutant as well as nonbinder mutant etc.

iii. Fyn SH3 domain and binder F29 dissociation constant $\left(K_{D}\right)$ Calculation-Quantified band intensities of pulled-down His-tagged Fyn SH3 domains (75nM to $1.25 \mu \mathrm{M})$ from immunoblot (given in Fig. 1e) has been used to calculate the Dissociation constant $\left(\mathrm{K}_{\mathrm{D}}\right)$. The data from three such independent experiments were analysed using GraphPad Prism-5 where a rectangular hyperbola saturation binding curve was plotted (as shown in Fig. 1f). In the graph, the curve shows saturation at a given concentration of ligand. This has been used to determine the $\mathrm{B}_{\max }$ and the following standard equation for "one-site specific" binding has been used to calculate the $\mathrm{K}_{\mathrm{D}}$.

$$
\mathrm{Y}=\frac{\mathrm{B}_{\max } * \mathrm{X}}{\mathrm{K}_{\mathrm{D}}+\mathrm{X}} \ldots \ldots \ldots \ldots \text {........ Supplementary Equation } 1
$$

\section{Wherein-}

$\mathrm{X}$ is ligand concentration (Fyn $\mathrm{SH} 3, \mathrm{nM}$ )

$\mathrm{Y}$ is amount of bound protein

$\mathrm{B}_{\text {maxis }}$ the maximum binding capacity (same units as the $\mathrm{Y}$-axis)

$\mathrm{K}_{\mathrm{D}}$ is the equilibrium dissociation constant (same units as the $\mathrm{X}$-axis, concentration).

\section{Protein Purification for NMR studies}

Fyn SH3 domain was encoded in a pET-14b plasmid, so that a 6X-His-SUMO-ppx-SH3 fusion protein can be produced. The plasmid was transformed into E. coli BL21-(DE3) cells, and grown in LB to produce unlabeled protein. ${ }^{13} \mathrm{C},{ }^{15} \mathrm{~N}$-labeled protein was produced by growing the $E$. coli in vitamin supplemented M9 minimal media with ${ }^{13} \mathrm{C}_{6} \mathrm{H}_{12} \mathrm{O}_{6}$ and ${ }^{15} \mathrm{NH} 4 \mathrm{Cl}$ as the sole source for carbon and nitrogen, respectively. Labeled/Unlabeled Fyn-SH3 domain was purified as follows: Cells were grown up to OD $\sim 0.8$, followed by induction with $1 \mathrm{mM}$ IPTG. Four hours post induction, cells were harvested by centrifugation at $4^{\circ} \mathrm{C}$, then washed once with Buffer-B (50mM sodium phosphate buffer, $\mathrm{pH} 8.0,300 \mathrm{mM} \mathrm{NaCl})$, supplemented with $0.1 \%$ $(\mathrm{v} / \mathrm{v})$ Triton $\mathrm{X}-100)$, and then stored at $-80^{\circ} \mathrm{C}$. Frozen cells were re-suspended in Buffer-B 
supplemented with $2 \mathrm{mM}$ phenylmethylsulfonyl fluoride (1:10, wt./vol.). The suspension was sonicated at $4{ }^{\circ} \mathrm{C}$ for $30 \mathrm{~min}$ for lysis, followed by centrifugation at $4^{\circ} \mathrm{C}$. The resultant supernatant was allowed to bind Nickel beads (Thermo Fisher), washed with $20 \mathrm{mM}$ imidazole, and eluted with 100-200 mM imidazole. Fractions containing the fusion protein were pooled, and the imidazole was removed by dialysis against Buffer-B for overnight at $4{ }^{\circ} \mathrm{C}$.

The fusion protein $(3 \mathrm{mg})$ was cleaved by Precission Protease $(1 \mathrm{mg})$ in an overnight $1 \mathrm{ml}$ reaction Buffer-B under mild rotating conditions at $4^{\circ} \mathrm{C}$. Cleaved Fyn-SH3 was purified by gelfiltration. Fyn-SH3 fractions were pooled and dialyzed against Buffer-C $(50 \mathrm{mM}$ sodium phosphate buffer, $\mathrm{pH} 8.0$ ) overnight at $4^{\circ} \mathrm{C}$. The dialyzed protein was applied to a Mono $\mathrm{Q}$ column equilibrated in Buffer-C. The protein was eluted with a linear gradient from 0 to $100 \%$ of Buffer-D (50mM sodium phosphate buffer, $\mathrm{pH} 8.0$ with $500 \mathrm{mMNaCl}$ ). Fractions containing pure Fyn SH3 were pooled and concentrated after dialysis in Buffer-B (50mM sodium phosphate buffer, $\mathrm{pH} 8.0,300 \mathrm{mM} \mathrm{NaCl})$.

p-GST-ppx-L $15-\mathrm{F} 29$ plasmid was transformed into E. coli BL21 (DE3) cells. The proteins were over-expressed in suitable media as given above. Post lysis, the supernatant was passed through a column of glutathione-Sepharose beads (GE-Healthcare) in Buffer-C. After extensive washing, the bound GST-ppx- $\mathrm{L}_{15}-\mathrm{F} 29$ fusion protein was eluted with $15 \mathrm{mM}$ glutathione in Buffer-C. Fractions containing the fusion protein were pooled and allowed dialyze overnight at $4^{\circ} \mathrm{C}$ in Buffer-C. The fusion protein was cleaved by Precission Protease. Cleaved F29 was purified by size exclusion chromatography. Fractions containing the F29 were pooled and concentrated up to1mM for NMR studies.

\section{Pull-down of total Endo-/ exogenously expressed-Fyn kinase using F29 binder}

Glutathione sepharose beads saturated with GST-F29 and GST protein (bait protein) were used to pull-down the endogenous and exogenously expressed Fyn kinase (prey protein, regulatable; WT as well as open active CA) from the cell lysate of HEK-293T cells. We have followed an established protocol $^{2}$ with modifications as described below.

(a) Bait protein preparation-The pGEX-6P-1 vector having ORFs of GST as well as fusion protein GST-F29 were transformed inE. coli BL21 competent cells. Single transformed bacterial colony was grown as pre-inoculum for 3 to $4 \mathrm{hr}$ in a $3 \mathrm{ml}$ of Luria-broth having ampicillin $(100 \mu \mathrm{g} / \mathrm{ml})$ in a $50 \mathrm{ml}$ conical screw cap tube at $37^{\circ} \mathrm{C}, 200 \mathrm{rpm}$ shaker. Grown preinoculums was transferred to a $100 \mathrm{ml}$ glass flask having $20 \mathrm{ml}$ Luria-broth and ampicillin and further grown for $2 \mathrm{hr}$. The cells were induced with IPTG $(500 \mu \mathrm{M})$ at OD=0.8 for $3 \mathrm{hr}$ for protein expression. Cells were harvested by centrifugation at 7,000 $\times \mathrm{g}$ and re-suspended in 1.5 $\mathrm{ml}$ of ice-cold lysis/wash buffer (1X PBS pH= 7.4, 5\% glycerol) followed by sonication with an ultrasonic processor on ice, for a total 3 cycles constituting of 12 pulses of $3 \mathrm{~s}$ ON time and 5 s OFF time at $40 \%$ amplitude. The cell-homogenate thus obtained was centrifuged at 20,000 $\times \mathrm{g}$ for $35 \mathrm{~min}$ at $4^{\circ} \mathrm{C}$ to yield clear cell-free extract (CFE). The total protein was estimated from CFE using BCA Protein assay kit (Pierce). The CFE was used to saturate the Glutathione sepharose beads and further used in pull-down experiments.

\section{(b) Endogenous kinase pull-down}

i. Cell culture and whole cell lysate- HEK-293T cells were cultured in $100 \mathrm{~mm}$ plate $\left(\sim 10^{6}\right.$ cells $)$ and grown for $48 \mathrm{~h}$ at $37^{\circ} \mathrm{C}$ in $5 \% \mathrm{CO}_{2}$ incubator. Plates were allowed to reach $90 \%$ confluency. From the plates, cells were washed with 1X PBS and scrapped 
gently in $600 \mu \mathrm{L}$ of RIPA lysis and extraction buffer cocktail $(10 \mathrm{ml}$ cocktail includes 1X Pierce RIPA buffer, 1 tablet of Pierce Phosphatase Inhibitor and 1 tablet of cOmplete mini Protease Inhibitor). The cells were centrifuged at 20000 x g for $20 \mathrm{~min}$ at $4^{\circ} \mathrm{C}$ to remove the cellular debris.

ii. Bead pre-clearance-The whole cell lysate (step $i$ ) was mixed with $50 \mu 1$ of glutathione sepharose beads and incubated at room temperature under mild $360^{\circ}$ rotating conditions $(5 \mathrm{rpm})$ for $2 \mathrm{~h}$. After centrifugation at 7,000 $\times \mathrm{g}$ for $1 \mathrm{~min}$, the pre-cleared cell lysate was taken and total protein was estimated by BCA assay.

iii. Bead incubation with bait protein- Bacterial CFE containing fusion bait protein GSTF29 or GST (control) was incubated with glutathione sepharose beads $(50 \mu \mathrm{l}$ beads with $\sim 7.5 \mathrm{mg}$ of $\mathrm{CFE}$ ) under mild $360^{\circ}$ rotating conditions $(5 \mathrm{rpm})$ for $2 \mathrm{~h}$ at room temperature in a low protein-binding micro-centrifuge tube (Eppendorf). The saturated beads were centrifuged at7,000 $\times \mathrm{g}$ for $1 \mathrm{~min}$ at $4^{\circ} \mathrm{C}$ and washed with 4 times with $1 \mathrm{ml}$ of lysis/wash buffer.

iv. Pull-down- Protein-saturated glutathione sepharose beads $(\sim 50 \mu \mathrm{l})$ were incubated with a total of $2.0 \mathrm{mg}$ of pre-cleared cell lysate (from step ii) for $2 \mathrm{~h}$ under mild rotation at room temperature. The unbound lysate was removed after centrifugation at 7,000 $\times \mathrm{g}$ for $1 \mathrm{~min}$. The beads were washed thrice with $1 \mathrm{X}$-PBS containing protease and phosphatase inhibitors $+0.1 \%$ NP40 under mild rotating conditions for $5 \mathrm{~min}$ at $4^{\circ} \mathrm{C}$.

$\mathrm{v}$. Immunoblot assay -The remaining beads were incubated with $25 \mu \mathrm{l}$ of $1 \mathrm{X}$ Laemmli buffer and denatured for $10 \mathrm{~min}$ at $98^{\circ} \mathrm{C}$. Using the extracted sample, we have performed the SDS (8\%) gel electrophoresis. The gel region spanning 29 to $43 \mathrm{KDa}$ region (bait protein) was stained with Coomassie blue dye, however 53 to $90 \mathrm{kDa}$ region (prey protein) was used for immunoblot using anti-Fyn antibody (1:500). The expression level of Fyn kinase was also analysed from $50 \mu \mathrm{g}$ of pre-cleared cell lysate in a separate immunoblot experiment using anti-Fyn antibody. Here $\beta$-actin antibody was used as loading control.

\section{(c) Exogenous Fyn Kinase pull-down}

i. $\quad$ Transfection, over-expression and whole-cell protein isolation-HEK-293T cells were cultured in $60 \mathrm{~mm}$ plate as described earlier. The cells were transfected with Fyn (WT or CA) DNA construct $(1.5 \mu \mathrm{g})$ using Lipofectamine LTX-DNA transfection reagents (Invitrogen) and incubated for $24 \mathrm{~h}$ at $37^{\circ} \mathrm{C}$ in $5 \% \mathrm{CO}_{2}$ incubator. The whole cell protein isolation was performed as described above.

ii. The steps used in pull-down experiment were kept identical as described above, except here, for each case only $25 \mu 1$ of glutathione sepharose beads were used to saturate 3.75 $\mathrm{mg}$ of CFE having bait protein GST-F29 or GST proteins. Here a total of $1.0 \mathrm{mg}$ of bead-pre-cleared lysate of HEK293T expressing WT or CA Fyn has been used. All subsequent immunoblotting steps were identical as described above.

\section{Fyn knock-down (KD) using retrovirus-mediated RNA interference}

The endogenous Fyn kinase protein of HEK-239T cells was knocked-down (KD) using Retrovirus-mediated RNA interference (sh-RNA) as described earlier ${ }^{3}$. pRetroSuper-shFyn construct has been used for endogenous Fyn knock-down which was a gift from Dr. Joan Massague (Addgene plasmid \# 26985). In this case the Fyn kinase (XM_017010653.1) 3'UTR region: 5'-GAACTTCCATGGCCCTCAT-3' was used as shRNĀtarget sequence. For scrambled shRNA control, we have used pSUPERretropuroScrshRNA, which was a gift from John Gurdon (Addgene plasmid \# 30520) where the scrambled shRNA sequence: 5'GCGAAAGATGATAAGCTAA-3'was used. For packaging of the retrovirus we have used 
AmphoPack-293 Cell Line (Gift from Dr Reety Arora, NCBS). 60-70\% confluent AmphoPack-293 cells (grown in 10cm culture plate, with $10 \mathrm{ml}$ DMEM supplemented with $10 \%$ FBS) were transfected with $10 \mu \mathrm{g}$ of shRNA DNA construct using Lipofectamine LTX and Plus reagent according to the manufacturer's instruction (Thermo-Fisher Scientific) in BSL2. Post-transfection the spent media containing packaged retrovirus were collected at 48 and $72 \mathrm{~h}$. The collected media $(\sim 18 \mathrm{ml})$ was filtered using $0.45 \mu \mathrm{m}$ filter and used to infect the HEK-293T cells (10 cm plate, 70-80 \% confluent, grown in DMEM and 10\% FBS) in presence of polybrene $(10 \mu \mathrm{g} / \mathrm{ml})$. The plate was further incubated for $24 \mathrm{~h}$ and replaced with fresh media having $4 \mu \mathrm{g} / \mathrm{ml}$ of puromycin (Sigma). After $48 \mathrm{~h}$, only $\sim 3$ to 4 cells (per $10 \mathrm{~cm}$ plate) were found viable in both cases. The media containing puromycin was changed as per requirement until these cells reached $\sim 90 \%$ confluency. Finally, the puromycin selected cells were passaged in the media without puromycin and grown. The cells were tested for mycoplasma and found to be negative. The final batches from each case were frozen and stored in liquid N2. The knockdown of Fyn kinase was verified by immunoblot using specific antibodies.

\section{Probing extracellular signal-regulated kinases (ERK) phosphorylation}

Earlier it has been shown that downstream ERK phosphorylation can be regulated through/is sensitive to Src family kinase 'Fyn' signalling ${ }^{4}$. Therefore, ERK phosphorylation offers a sensitive measure of the signalling state. Here we aim to show-(a) cellular Fyn levels regulate downstream ERK signaling, (b) FynSensor Fyn is able to rescue downstream ERK signaling in Fyn-knockdown cells efficiently. (c) FynSensor, labelled Fyn and F29 binder do not perturb downstream ERK signaling in multiple cell lines.

a) Cellular Fyn levels regulate downstream signaling-In order to show that ERK phosphorylation is sensitive to Fyn signaling, we used stable cells expressing Fyn shRNA (Fyn KD HEK cells) as well as scrambled shRNA (control HEK cells) in this experiment. The cells were seeded at a density of $\sim 3 \mathrm{X} 10^{5} / \mathrm{ml}$ in $35 \mathrm{~mm}$ tissue culture dishes. After $24 \mathrm{~h}$, the cells were incubated in media containing $1 \%$ serum. $16 \mathrm{~h}$ post incubation, the cells were washed with 1X DPBS and lysed in $200 \mu$ of RIPA lysis buffer. The cell lysates were resolved on $10 \%$ SDS gel followed by transfer on a PVDF membrane. Blot region corresponding to 54 to $90 \mathrm{kDa}$ was probed with anti-Fyn antibody, 29 to $54 \mathrm{kDa}$ with anti-p44/42 MAPK (Erk1/2) antibody and anti-Phosphop44/42 MAPK (Erk1/2) (Thr202/Tyr204) antibody. Vinculin was used as loading control (116kDa). The band intensities from at least three such blots were analysed and the levels of phospho-/total ERK were compared using GraphPad Prism.

b) FynSensor Fyn is able to rescue downstream ERK signaling in Fyn-knockdown cells efficiently-In the FynKD-HEK cells, the ERK phosphorylation rescue assay was performed by transiently expressing vector (pTriex4 Neo) alone or unlabeled WT Fyn kinase or, FynSensor \{FRET donor (mCer-Fyn) + FRET acceptor (myr-mVenus-F29) in reduced serum (1\%) media for $18 \mathrm{~h}$. Using cell lysates from these different conditions, we have performed the immunoblot experiment using specific antibodies as described above. The band intensities from at least three such blots were analysed and the levels of phospho-/total ERK were compared using GraphPad Prism.

c) FynSensor, labeled Fyn and F29 binder do not perturb downstream ERK signalling in multiple cell lines-

i) U2OS cells - Cells were seeded at a density of $\sim 3 \mathrm{X} 10^{5} / \mathrm{ml}$ cells $/ \mathrm{ml}$ in $35-\mathrm{mm}$ culture dishes containing $2 \mathrm{ml}$ media. After $24 \mathrm{~h}$, cells were transiently transfected with vector, or myr-mVenus or myr-mVenus-F29 (FRET acceptor) or Fyn kinase donor (mCer-Fyn) or FynSensor. After $18 \mathrm{~h}$ of transient protein expression, cells 
were lysed using RIPA buffers. The lysates were resolved on to 10\% SDS gel followed by transfer on a PVDF membrane. Blot region corresponding to 54 to 90kDa was probed with anti-Fyn antibody, 29 to $54 \mathrm{kDa}$ with anti-p44/42 MAPK (Erk1/2) antibody and anti-Phospho-p44/42 MAPK (Erk1/2) (Thr202/Tyr204) antibody. Vinculin antibody was used as loading control (116kDa). The band intensities from at least 3 such blots were analysed and the levels of phospho/total ERK were compared using GraphPad Prism.

ii) Mouse myoblast C2C12 cells - Cells were seeded at a density of $\sim 3 \times 10^{5} / \mathrm{ml}$ cells $/ \mathrm{ml}$ in $35-\mathrm{mm}$ culture dishes containing $2 \mathrm{ml}$ media. After $24 \mathrm{~h}$, cells were transiently transfected with vector, or unlabelled wtFyn or myr-mVenus-F29 (FRET acceptor) or Fyn kinase donor (mCer-Fyn) or FynSensor. After 18h of transient protein expression, cells were lysed using RIPA buffers. The lysates were resolved on to $10 \%$ SDS gel followed by transfer on a PVDF membrane. Blot region corresponding to 54 to $90 \mathrm{kDa}$ was probed with anti-Fyn antibody, 29 to $54 \mathrm{kDa}$ with anti-p44/42 MAPK (Erk1/2) antibody and anti-Phosphop44/42 MAPK (Erk1/2) (Thr202/Tyr204) antibody. $\beta$-actin was used as loading control $(43 \mathrm{kDa})$.

\section{TIRF microscopy for visualizing Fyn localization}

U2OS cells transiently expressing the FynSensor (WT-mCer-Fyn+mVenus-F29) were plated on FN coated glass bottomed dish and imaged on the Olympus IX83 inverted microscope coupled to the cellTIRF® module. Cells were excited using the $445 \mathrm{~nm}$ laser and imaged using a 100X objective (Olympus UAPON 100X oil TIRF objective, NA=1.49) at a penetration depth of $100 \mathrm{~nm}$. Images were captured on the Evolve 512 Delta EMCCD camera at an exposure of 400ms. Images were acquired using the Olympus cellSens software and processed using Fiji.

\section{In-vitro biosensor characterization}

The over-expression, protein integrity and activity of the biosensor was analysed in live cells. Following methods have been adapted-

i. Transfection- HEK-293T cells were cultured in $35 \mathrm{~mm}$ plate. Cells were transiently transfected with Lipofectamine LTX and plus reagent according to the manufacturer's instruction (Thermo Fisher Scientific).

ii. Whole cell fluorescence measurement- After $18 \mathrm{~h}$ post transfection, cells were washed gently with $1 \mathrm{X}$ DPBS (Invitrogen) and re-suspended in 1ml 1X DBPS. The live cell suspension was used to measure the excitation and emission spectra of fluorescent protein using spectrofluorometer (Fluorolog, Horiba). The slit width and PMT voltage was kept $2 \mathrm{~nm}$ and $950 \mathrm{~V}$, respectively. The excitation and emission wavelength was kept identical to respective fluorescent protein as mentioned in methods.

iii. Whole cell protein isolation- the cells from suspension (step ii) were harvested by gentle centrifugation $\left(1000 \mathrm{x} \mathrm{g}\right.$ for $5 \mathrm{~min}$ at $4^{\circ} \mathrm{C}$ ) and incubated in presence of $\sim 300 \mu \mathrm{l}$ of RIPA lysis and extraction buffer cocktail in $-80^{\circ} \mathrm{C}$ for overnight. The cells were lysed by gentle vortexing and centrifuged at $2000 \mathrm{x}$ g for $20 \mathrm{~min}$ at $4^{\circ} \mathrm{C}$ in order to collect the whole cell lysate.

iv. Immunoblot assay- Protein was estimated from cell lysate (step iii) using BCA protein assay kit (Pierce). Cell lysate $(50 \mu \mathrm{g})$ from samples was incubated in $1 \mathrm{X}$ laemmli buffer and denatured for $10 \mathrm{~min}$ at $95^{\circ} \mathrm{C}$. Using these samples, we performed the SDS gel ( 8 
$\%)$ electrophoresis. The proteins from SDS gel were transferred onto PVDF membrane (Invitrogen, $0.2 \mu \mathrm{m}$ ) at $100 \mathrm{~V}$ for $90 \mathrm{~min}$ at $4^{\circ} \mathrm{C}$ in $1 \mathrm{X}$ transfer buffer. After complete transfer the PVDF membrane was incubated in blocking buffer (5\% non-fat dry milk (Blotto) in 1X-TBST for $1 \mathrm{~h}$ at room temperature on a shaker. The blot regions having protein of interests were incubated in hybridization bags with their specific primary antibody (5\% BSA in 1X TBST) buffer at $4{ }^{\circ} \mathrm{C}$, overnight under mild shaking conditions. These membranes were washed stringently with 1X-TBST and incubated with anti-mouse or rabbit IgG, HRP-linked secondary antibody (1:3000) at room temperature for $1 \mathrm{hr}$. The membranes were then washed 4 times with 1X-TBST and imaged using Image-Quant LAS 4000 (GE-Healthcare) in luminol-based enhanced chemiluminescent reagent. The band intensity of blots was analysed using Image-J (NIH).

\section{Fibronectin coating of confocal dishes}

The glass bottom dishes were coated with $10 \mu \mathrm{g} / \mathrm{ml}$ of fibronectin solution. Briefly, the $1 \mathrm{mg} / \mathrm{ml}$ stock was diluted to the desired concentration in DPBS and the solution was added to the plates and incubated at $37^{\circ} \mathrm{C}$ for an hour. Post incubation plates were washed with DPBS and stored for immediate use at $4^{\circ} \mathrm{C}$.

\section{Cell treatment and Image acquisition for SU6656-APB experiment}

The Acceptor photo-bleaching experiment on SU6656 treated cells was carried on the Olympus FV1000 confocal microscope using a 63X oil immersion objective. FRET-donor (mCer-Fyn) was excited using the $405 \mathrm{~nm}$ laser and emission from (425-500) nm was collected. Acceptor (myr-mVenus-F29) was excited using the 515nm laser and emission from (519-619) nm was collected. For bleaching the acceptor, the $515 \mathrm{~nm}$ laser was used at a high laser dose $(1.38 \mathrm{~mW}$ for 27 seconds). Post bleaching, both the donor and acceptor channel images were acquired. The data analysis was done as described in the methods section. Cells transfected with FynSensor were serum starved and plated as mentioned in methods. Cells were then treated with $2 \mu \mathrm{M}$ of SU6656 inhibitor ( $2 \mathrm{mM}$ stock in 100\% DMSO). Post inhibitor addition, bleaching of the acceptor was carried on. DMSO controls were treated and imaged in the same way.

\section{Immunofluorescence Assay}

i) Coverslip coating - A 24-well plate containing glass coverslips $(10 \mathrm{~mm}$ diameter) was used to attach the cells. The coverslips were washed with $1 \mathrm{X}$ DBPS and coated with Fibronectin (FN, $10 \mu \mathrm{g} / \mathrm{ml}$, in DPBS, 300 $\mu 1$ for one coverslip in a well). The plate was incubated for $60 \mathrm{~min}$ at $37^{\circ} \mathrm{C}$. The extra fibronectin was removed by aspiration and coverslips were washed with DPBS. Plate was either stored at $4^{\circ} \mathrm{C}$ or used immediately for cell attachment.

ii) Cell attachment-70-90\% confluent cells (transfected or un-transfected), were trypsinized $(0.25 \%$ trypsin) and suspension was made in appropriate media. Approximately 50000 cells were seeded in $300 \mu 1$ culture media onto fibronectin coated coverslips (in the 24-well plate). Cells were allowed to attach for $40 \mathrm{~min}$ in an incubator prior to further processing.

iii) Fixation- Attached cells in the wells were washed once with $300 \mu 1$ of 1 X DPBS. $300 \mu 1$ of paraformaldehyde solution (4\% in 1X DPBS having $10 \%$ methanol) was added as fixative reagent and incubated under gentle rocking condition for 
$20 \mathrm{~min}$ at room temperature. The cells were then washed thrice with $300 \mu \mathrm{l}$ with $1 \mathrm{X}$ DPBS.

iv) Permeabilization - To each well, $300 \mu$ l of permeabilization solution $(0.1 \%$ Triton-X100 in 1X DPBS) was added and incubated for 15 min under gentle

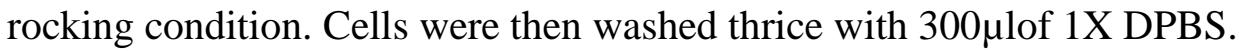

v) Blocking- To each well, 300 $\mu 1$ of blocking solution (1\% BSA in 1X DBPS and $1 \%$ FBS) was added and incubated for $60 \mathrm{~min}$ at room temperature under gentle rocking condition.

vi) $1^{\circ}$ Antibody co-staining - To each well, $200 \mu 1$ of $1^{\circ}$ Antibody [Anti-Fyn Mouse monoclonal antibody, Abcam \# ab3116 and Phospho-p44/42 MAPK (Erk1/2) (Thr202/Tyr204) Antibody CST \#9101; IF Grade) diluted in antibody dilution reagent $(0.05 \%$ tritonX100 in 1 X DBPS having 1\% BSA, Dilution 1:500, 1:100, respectively] was added and incubated at $4{ }^{\circ} \mathrm{C}$ overnight under gentle rocking condition. The following day, the cells were washed thrice with $300 \mu \mathrm{l}$ of $1 \mathrm{X}$ DBPS for 5 min each under gentle rocking condition.

vii) $2^{\circ}$ Antibody co-staining- To each well, $200 \mu$ l of secondary Antibody [Goat antimouse IgG secondary antibody, Alexa Fluor 647 conjugate, Invitrogen\# 21236 and Donkey anti-rabbit secondary antibody, Alexa Fluor 546 conjugate, Invitrogen \# 10040) diluted in antibody dilution reagent ( $0.05 \%$ triton-100 in $1 \mathrm{X}$ DBPS having 1\% BSA, at 1:500 dilutions] and incubated at room temperature under gentle rocking condition for $1 \mathrm{hr}$ in dark. Following that, cells were washed thrice with $300 \mu$ l of 1 X DBPS.

viii) Staining Nucleus-300 $\mu$ l of Hoechst 33342 nucleic acid stain $(1 \mathrm{mg} / \mathrm{ml})$ was added to a final dilution of $(1: 1000)$ in 1 XPBS to each well and incubated for 30 min under gentle rocking condition in dark. Following that, cells were washed thrice with $300 \mu 1$ of $1 \mathrm{X}$ DBPS for $2 \mathrm{~min}$.

ix) Mounting-Coverslips with fixed and stained cells were finally mounted onto a clean glass slide using $7 \mu \mathrm{l}$ of Mowiol mounting medium and stored for 2 days for proper drying in dark. The slides were stored at $4^{\circ} \mathrm{C}$ and used for confocal microscopy.

\section{Fyn and ERK localization studies using Immunofluorescence Assay}

Post 16 h of transfection the HEK 293T Fyn KD (with FynSensor WT, or unlabeled WT Fyn) or un-transfected HEK293T control cells were starved for another $4 \mathrm{~h}$ in serum free media and then plated on glass coverslip coated with fibronectin $(10 \mu \mathrm{g} / \mathrm{ml})$ and allowed to attach for 40 min. The cells were then fixed and used for immunofluorescence studies (see methods above). Alexa Fluor-647 conjugated Fyn kinase protein complex was excited using $640 \mathrm{~nm}$ laser and Alexa Fluor-546 conjugated phospho-ERK protein complex was excited using $561 \mathrm{~nm}$ laser. In order to capture the spatial expression of Fyn and phospho-ERK, several optical slices along the $\mathrm{z}$-axis were collected. The slice with maximum intensity for the emission corresponding to $640 \mathrm{~nm}$ excitation channel (specific for Alexa Fluor-647 conjugated Fyn kinase protein) was chosen, its intensity value was measured using Fiji. From the same optical plane, the corresponding emission for $561 \mathrm{~nm}$ laser excitation (signal specific for Alexa Fluor-546 conjugated phospho-ERK channel) intensity was also measured. The data was analysed using GraphPad Prism and shown in Supplementary Fig. 15. 
1 Supplementary Table 1. List of constructs used in study.

\begin{tabular}{|c|c|c|c|c|}
\hline Name & Gene of Interest & Modifications in constructs & Product of fusion protein & Vector and Hosts \\
\hline p-SUMO-ppx-FynSH3 & FynSH3 domain & \multirow{4}{*}{$\begin{array}{l}\text { Thrombin cleavage site (LVPRGSH) } \\
\text { was replaced with Pre-Scission protease } \\
\text { cleavage site (LEVLFQGP) }\end{array}$} & 6X-His-SUMO-ppx-FynSH3 & pET14b, Bacteria \\
\hline p-SUMO-ppx-Src SH3 & Src SH3 domain & & 6X-His-SUMO-ppx-Src SH3 & pET14b, Bacteria \\
\hline p-SUMO-ppx-F29 & F29, Fyn SH3 binder & & 6X-His-SUMO-ppx-F29 & pET14b, Bacteria \\
\hline p-SUMO-ppx-Src SH3 T99D & Src SH3 domain mutant & & 6X-His-SUMO-ppx-Src SH3 T99D & pET14b, Bacteria \\
\hline p-GST-ppx-L $15-F 29$ & F29, Fyn SH3 binder & \multirow{3}{*}{$\begin{array}{l}\text { Pre-Scission protease cleavage site and } \\
\text { F29 ORF was fused with gene flexible } \\
\text { linker (GGGGS)3 }\end{array}$} & GST-ppx-L $15-F 29$ & pGEX6P1, Bacteria \\
\hline p-GST-ppx-L $15-F 29$ P41A & \multirow{2}{*}{ Fyn Binder point mutant } & & GST-ppx-L15-F29 P41A & pGX6P1, Bacteria \\
\hline p-GST-ppx-L 15 -F29 R33A & & & GST-ppx-L $15-F 29$ R33A & pGX6P1, Bacteria \\
\hline p-GST-ppx-Fyn SH3 & Fyn SH3 domain & none & GST-ppx-Fyn SH3 & pGX6P1, Bacteria \\
\hline p-GST-ppx-Src SH3 & Src SH3 domain & none & GST-ppx-Src SH3 & pGX6P1, Bacteria \\
\hline p-GST-ppx-Yes SH3 & Yes SH3 domain & none & GST-ppx-Yes SH3 & pGX6P1, Bacteria \\
\hline p-FynWT & h-FynWT & \multirow[t]{2}{*}{ none } & Fyn Kinase WT & pTriex4neo, Dual \\
\hline p-FynCA & h-FynCA Y531F & & Fyn kinase CA $(\mathrm{Y} 531 \mathrm{~F})$ & pTriex4neo, Dual \\
\hline $\begin{array}{l}\text { p-UD- } \mathrm{L}_{15}-\mathrm{mCerulean}^{-} \mathrm{L}_{15-} \\
\text { FynWT }\end{array}$ & Labeled h-FynWT & \multirow{2}{*}{$\begin{array}{l}\text { mCerulean was inserted between Ud } \\
\text { and SH3 domain of Fyn flanked by } \\
\text { linker (GGGGS)3 }\end{array}$} & UD-L $\mathrm{L}_{15}$-mCerulean-L $\mathrm{L}_{15}$-FynWT & pTriex4neo, Dual \\
\hline p-UD-L $\mathrm{L}_{15}$-mCerulean- $\mathrm{L}_{15}$-FynCA & Labeled h-FynCA & & UD-L $_{15}$-mCerulean-L ${ }_{15}$-FynCA Y531F & pTriex4neo, Dual \\
\hline p-FynWT- $\mathrm{L}_{15}$-mCerulean & Labeled h-FynWT & $\begin{array}{l}\text { mCerulean was fused after Fyn flanked } \\
\text { by flexible linker (GGGGS)3 } \\
\end{array}$ & Fyn- $\mathrm{L}_{15}$-mCerulean WT & pTriex4neo, Dual \\
\hline p-mCerulean & mCerulean & none & mCerulean & pTriex4neo, Dual \\
\hline p-mVenus & mVenus & none & mVenus & pTriex4neo, Dual \\
\hline p-mVenus- $\mathrm{L}_{15}-\mathrm{F} 29$ & \multirow{4}{*}{ FynSH3 domain binder } & \multirow{2}{*}{$\begin{array}{l}\text { mVenus and Binder ORF was fused } \\
\text { with flexible linker (GGGGS) } 3\end{array}$} & mVenus-L ${ }_{15}-\mathrm{F} 29$ & pTriex4neo, Dual \\
\hline p-mVenus-L ${ }_{15}-\mathrm{F} 29$ P41A & & & mVenus-L $15-\mathrm{F} 29 \mathrm{P} 41 \mathrm{~A}$ & pTriex4neo, Dual \\
\hline p-myr-mVenus- $\mathrm{L}_{15}-\mathrm{F} 29$ & & \multirow{3}{*}{$\begin{array}{l}\text { myristoyl group (MGSSKSKPKDPS) } \\
\text { was fused before mVenus }\end{array}$} & $m y r-\mathrm{mVenus}-\mathrm{L}_{15}-\mathrm{F} 29$ & pTriex4neo, Dual \\
\hline p-myr-mVenus-L ${ }_{15}-\mathrm{F} 29$ P41A & & & myr-mVenus-L $\mathrm{L}_{15}-\mathrm{F} 29 \mathrm{P} 41 \mathrm{~A}$ & pTriex4neo, Dual \\
\hline p-myr-mVenus & mVenus & & myr-mVenus & pTriex4neo, Dual \\
\hline
\end{tabular}


3 Supplementary Table 2. Lists of primers used in study.

\begin{tabular}{|c|c|c|c|}
\hline $\mathbf{S N}$ & Name & Sequence (5'-----3') & Specifications \\
\hline 1 & $\mathrm{P} 2 \mathrm{R}$ & GGGCCCCTGGAACAGAACTTCCAGACCGGAACTTGCCGCCGCGTACG & \multirow{3}{*}{$\begin{array}{l}\text { Underline is overlapping } \\
\text { sequence of prescission protease } \\
\text { cleavage site (LEVLFQGP) }\end{array}$} \\
\hline 2 & P3F-Fyn & CTGGAAGTTCTGTTCCAGGGGCCCATGTTCGTTGCTCTGTATG & \\
\hline 3 & P3F-Src & CTGGAAGTTCTGTTCCAGGGGCCCATGACCTTCGTCGCCCTGTATGAC & \\
\hline 4 & Src SH3 T99D FP & CGTACCGAAGATGATCTGAGCTTTAAAAAAGGTGAACGCC & \multirow{2}{*}{$\begin{array}{l}\text { Underline region is codon for } \\
\text { aspartic acid }\end{array}$} \\
\hline 5 & Src SH3 T99D RP & GCTCAGATCATCTTCGGTACGGGATTCATAG & \\
\hline 6 & FB Nde FP & GGCTCGAGCATATGGCGACCGTGAAAT & \multirow{2}{*}{$\begin{array}{l}\text { Underline are restriction sites for } \\
N d e \mathrm{I} \text { and } \mathrm{Bam} \mathrm{H} 1\end{array}$} \\
\hline 7 & FB BamH1 RP & AAGCGGATCCTTATTTTTTCTGTTTTTCC & \\
\hline 8 & pGEXPS15L FP & CTGTTCCAGGGGCCCCTGGGATCCGGTGGAGGCGGTTCAGGCGGAG & \multirow{2}{*}{$\begin{array}{l}\text { Underlines are overlap regions } \\
\text { with pGEX6P1. Bam } \mathrm{H} 1 \text { and } \\
\text { XhoI site are shown in italics. }\end{array}$} \\
\hline 9 & F29 pGEX RP & GCGGCCGCTCGAGTCGACCTTATTTTTTCTGTTTTTCCAGCTTCTGCAGCAG & \\
\hline 10 & F29 P41A FP & ATCTGGGCGGCGGCAAAGCGGGCTTTGGCGTCGTGAGCGA & \multirow{4}{*}{ Underline is codon for alanine } \\
\hline 11 & F29 P41A FP & CGCCAAAGCCCGCTTTGCCGCCGCCCAGATCATATCTAAA & \\
\hline 12 & F29 R33A FP & AGCGATTTTT TTTGCGTATG ATCTGGGCGG CGGC & \\
\hline 13 & F29 R33A RP & CCCAGATCATACGCAAAAAAAATCGCTTTGCCGT & \\
\hline 14 & 15L-BC-FynSH3 FP & GGCGGAGGTGGCTCTGGCGGTGGCGGATCGACAGGAGTGACACTCTTTGTGGCCC & \multirow{4}{*}{$\begin{array}{l}\text { Yellow highlighted are coding } \\
\text { sequence of flexible linker. } \\
\text { Underlined region are for B, } \\
\text { italic for C and regular for A }\end{array}$} \\
\hline 15 & UD-15L-AB RP & AGAGCCACCTCCGCCTGAACCGCCTCCACCTCCTCCTCTCGTACGCAAGGTCCCCG & \\
\hline 16 & 15L-BC-CER-VEN FP & GGCGGAGGTGGCTCT $G G C G G T G G C G G A T C G A T G G T G A G C A A G G G C G A G G A G C T G$ & \\
\hline 17 & CER-VEN 15L AB RP & AGAGCCACCTCCGCCTGAACCGCCTCCACCCTTGTACAGCTCGTCCATGCCGAG & \\
\hline 18 & FynY527F FP & CAGAGCCCCAGTTCCAACCTGGTGAAAACC & \multirow{2}{*}{$\begin{array}{l}\text { Underline is codon for } \\
\text { Phenylalanine }\end{array}$} \\
\hline 19 & FynY527F RP & CACCAGGTTGGAACTGGGGCTCTGTCGCGG & \\
\hline 20 & UdFynNcoI FP & CAAAGGAGATATACCATGGGCTGTGTGCAATGTAAGG & \multirow{2}{*}{$\begin{array}{l}\text { Underline are restriction sites for } \\
N c o I \text { and } B a m \mathrm{H} 1 \text { enzymes }\end{array}$} \\
\hline 21 & FynBamH1 RP & CTGAGAATTCGGATTCTTACAGGTTTTCACCAGGTTGGTACTGGGGCTC & \\
\hline 22 & CER-VEN NcoI FP & CAAAGGAGATATACCATGGTGAGCAAGGGCGAGGAGCTG & Underline is $N c o$ I site \\
\hline 23 & CER-VEN BamH1 RP & CTGAGAATTCGGATTCTTATTGTACAGCTCGTCCATGCCGAG & Underline is BamH1 site \\
\hline 24 & 15L-BC-F29 FP & GGCGGAGGTGGCTCT $G G C G G T G G C G G A T C G A T G G C G A C C G T G A A A T T T A A A T A T A A A G G C G$ & Same for SN 18 primer \\
\hline 25 & F29 BamH1 RP & GCCGAGATCTGAGAATTCGGATCCTTATTTTTTCTGTTTTTCCAGCTTCTGCAGCAG & Underline BamH1 site \\
\hline 26 & Myr CER-VEN FP & ATGGGCAGTAGCAAGAGCAAGCCTAAGGACCCCAGC ATGGTGAGCAAGGGCGAGGAG & \multirow{2}{*}{$\begin{array}{l}\text { Grey highlighted are coding } \\
\text { sequence of myristoyl group tag }\end{array}$} \\
\hline 27 & PTriexMyr RP & $\begin{array}{l}\text { GCTGGGGTCCTTAGGCTTGCTCTTGCTACTGCCCATGGTATATCTCCTTTGATTGTAAATAA } \\
\text { AATGTAATTACAGTATAG }\end{array}$ & \\
\hline
\end{tabular}


bioRxiv preprint doi: https://doi.org/10.1101/726133; this version posted August 5, 2019. The copyright holder for this preprint (which was

not certified by peer review) is the author/funder, who has granted bioRxiv a license to display the preprint in perpetuity. It is made available under aCC-BY-NC-ND 4.0 International license.

5 Supplementary Table 3. Structural Statistics of Fyn-SH3/F29 complex.

\begin{tabular}{|l|l|}
\hline & Fyn-SH3/F29 \\
\hline Restraints & \\
\hline Unambiguous restraints & 06 \\
\hline Ambiguous restraints & 16 \\
\hline & \\
\hline Haddock Statistics & \\
\hline Cluster Size & 200 \\
\hline Haddock score & $-98.1( \pm 14.4)$ \\
\hline Van Der Waals Energy & $-56.9( \pm 11.6)$ \\
\hline Electrostatic Energy & $-235.4( \pm 43.2)$ \\
\hline Non-bonded energy & $-292.2( \pm 42.6)$ \\
\hline Surface area & $+1436.7( \pm 104.9)$ \\
\hline & \\
\hline Rmsd & \\
\hline Bond angles & 0.5 \\
\hline Bond lengths & 0.003 \\
\hline Rmsd & \\
\hline All backbone & 0.5 \\
\hline All heavy atoms & 0.7 \\
\hline MolprobityClashscore ${ }^{\mathrm{b}}$ & 1.53 \\
\hline & \\
\hline Ramachandran Map & $9.7 \%$ \\
\hline Most Favoured regions & $5.2 \%$ \\
\hline Allowed regions & \\
\hline Disallowed regions & \\
\hline
\end{tabular}

6

$7 \quad{ }^{\mathrm{a}}$ Calculated for an ensemble of 10 lowest energy structures.

$8 \quad{ }^{\mathrm{b}}$ Calculated for the lowest energy structure. 
Supplementary Table 4. Interactions observed in the Fyn-SH3:F29 complex

\begin{tabular}{|c|c|c|c|c|c|}
\hline \multicolumn{3}{|l|}{ Fyn-SH3 } & \multicolumn{3}{|l|}{ F29 } \\
\hline \multicolumn{6}{|c|}{ Hydrophobic Interactions within $5 \mathrm{~A}^{\circ}$} \\
\hline $\begin{array}{l}\text { Residue } \\
\text { Position }\end{array}$ & $\begin{array}{l}\text { Residue } \\
\text { type }\end{array}$ & Atom & $\begin{array}{l}\text { Residue } \\
\text { Position }\end{array}$ & $\begin{array}{l}\text { Residue } \\
\text { type }\end{array}$ & Atom \\
\hline 90 & LEU & & $26 *$ & TYR & \\
\hline 91 & TYR & & 26 & TYR & \\
\hline 119 & TRP & & 22 & PHE & \\
\hline 119 & TRP & & 31 & PHE & \\
\hline 119 & TRP & & 43 & PHE & \\
\hline 132 & TYR & & 43 & PHE & \\
\hline 134 & PRO & & 31 & PHE & \\
\hline 137 & TYR & & 26 & TYR & \\
\hline \multicolumn{6}{|c|}{ Side-chain: Main-chain hydrogen bonds } \\
\hline 96 & ARG & NH1 & 21 & VAL & $\mathrm{O}$ \\
\hline 96 & ARG & NH1 & 21 & VAL & $\mathrm{O}$ \\
\hline 96 & ARG & $\mathrm{NE}$ & 64 & LYS & $\mathrm{O}$ \\
\hline 119 & TRP & NE1 & 32 & PHE & $\mathrm{O}$ \\
\hline 136 & $\mathrm{ASN}$ & ND2 & 24 & THR & $\mathrm{O}$ \\
\hline 136 & ASN & ND2 & 24 & THR & $\mathrm{O}$ \\
\hline 136 & $\mathrm{ASN}$ & ND2 & 29 & GLN & $\mathrm{O}$ \\
\hline 136 & $\mathrm{ASN}$ & ND2 & 29 & GLN & $\mathrm{O}$ \\
\hline 137 & TYR & $\mathrm{OH}$ & 25 & ARG & $\mathrm{O}$ \\
\hline 116 & GLU & $\mathrm{O}$ & 8 & TYR & $\mathrm{OH}$ \\
\hline \multicolumn{6}{|c|}{ Side-chain: Side-chain hydrogen bonds } \\
\hline 118 & $\mathrm{ASP}$ & OD1 & 9 & LYS & $\mathrm{NZ}$ \\
\hline 93 & TYR & $\mathrm{OH}$ & 24 & THR & OG1 \\
\hline 99 & ASP & OD1 & 33 & ARG & NH1 \\
\hline 99 & ASP & OD1 & 33 & ARG & NH1 \\
\hline \multicolumn{6}{|c|}{ Aromatic Interactions } \\
\hline 91 & TYR & & 22 & PHE & \\
\hline 119 & TRP & & 26 & TYR & \\
\hline 119 & TRP & & 43 & PHE & \\
\hline 132 & TYR & & 43 & PHE & \\
\hline 137 & TYR & & 26 & TYR & \\
\hline
\end{tabular}

11 *The residues that are mutated from Sso7d (original scaffold) to F29 are marked in bold 
bioRxiv preprint doi: https://doi.org/10.1101/726133; this version posted August 5, 2019. The copyright holder for this preprint (which was

not certified by peer review) is the author/funder, who has granted bioRxiv a license to display the preprint in perpetuity. It is made available under aCC-BY-NC-ND 4.0 International license.

\section{Supplementary References}

14

15

16

17

18

19

1 Gera, N., Hussain, M. \& Rao, B. M. Protein selection using yeast surface display. Methods60, 15-26, doi:10.1016/j.ymeth.2012.03.014 (2013).

2 Sambrook, J. \& Russell, D. W. Detection of Protein-Protein Interactions Using the GST Fusion Protein Pulldown Technique. CSH protocols2006, doi:10.1101/pdb.prot3757 (2006).

3 Zhang, X. H. et al. Latent bone metastasis in breast cancer tied to Src-dependent survival signals. Cancer cell16, 67-78, doi:10.1016/j.ccr.2009.05.017 (2009).

4 Wary, K. K., Mariotti, A., Zurzolo, C. \& Giancotti, F. G. A requirement for caveolin1 and associated kinase Fyn in integrin signaling and anchorage-dependent cell growth. Cell94, 625-634 (1998). 
Legend for Supplementary Videos

Supplementary video 1: FRET response in human osteosarcoma cell-line, U2OS cells expressing non-binder mutant of FynSensor.

Live cell imaging of U2OS cells transfected with non-binder mutant of FynSensor before and after PDGF stimulation. Panel shows FRET index image using Fire LUT (Fiji) and scaled for ease of visualization. Individual images were acquired at $60 \mathrm{X}$ at an interval of $30 \mathrm{~s}$ and then stitched together to make a video (played at5 frames/second). Time stamp indicates elapsed time, "+PDGF" label shows the point at which PDGF was added to the cells. Colour bar shows intensity of FRET signal. No/very little FRET signal is seen from these cells and this FRET signal is not "enhanced" post PDGF stimulation. Scale bar $=10 \mu \mathrm{m}$.

\section{Supplementary video 2: Effect of FAK inhibition on Fyn activity in human osteosarcoma cell-line, U2OS cells.}

Live cell imaging of U2OS cells transfected with FynSensor before and after inhibition of FAK. Panel shows FRET index image using Fire LUT (Fiji) and scaled for ease of visualization. Individual images were acquired at $60 \mathrm{X}$ at an interval of $60 \mathrm{~s}$ as part of a multipoint-time-lapse imaging regime and then stitched together to make a video (played at 3 frames/second). Time stamp indicates elapsed time, "+FAK inhibitor" label shows the point at which FAK inhibitor was added to the cells. Colour bar shows intensity of FRET signal. Basal level of Fyn activity (unstimulated state) is highly localized in space. Post addition of inhibitor the Fyn activity in the cell is drastically attenuated. Scale bar $=10 \mu \mathrm{m}$.

\section{Supplementary video 3: Fyn activation in human osteosarcoma cell-line, U2OS} cells.

Live cell imaging of U2OS cells transfected with FynSensor plated on Fibronectin (FN). Panel shows FRET index image using Fire LUT (Fiji) and scaled for ease of visualization. Individual images were acquired at $60 \mathrm{X}$ at an interval of $60 \mathrm{~s}$ and then stitched together to make a video (played at 3 frames/second). Time stamp indicates elapsed time. Colour bar shows intensity of FRET signal. Basal level of Fyn activity (caused by integrin engagement) is spatially compartmentalized and shows temporal bursts of signals (activity pulses). Scale bar $=10 \mu \mathrm{m}$.

\section{Supplementary video 4: Fyn activity in U2OScells is modulated by PDGF.}

Live cell imaging of U2OS cells transfected with FynSensor before and after PDGF stimulation. Panel shows FRET index image using Fire LUT (Fiji) and scaled for ease of visualization. Individual images were acquired at $60 \mathrm{X}$ at an interval of $60 \mathrm{~s}$ and then stitched together to make a video (played at 3 frames/second). Time stamp indicates elapsed time, 
bioRxiv preprint doi: https://doi.org/10.1101/726133; this version posted August 5, 2019. The copyright holder for this preprint (which was

not certified by peer review) is the author/funder, who has granted bioRxiv a license to display the preprint in perpetuity. It is made available under aCC-BY-NC-ND 4.0 International license.

63

64

65

66

67

68

69

70

71

72

73

74

75

76

77

78

"+PDGF" label shows the point at which PDGF was added to the cells. Colour bar shows intensity of FRET signal. Basal level of Fyn activity (un-stimulated state) is spatially compartmentalized and post PDGF addition, FRET response is strengthened significantly from this "compartment/zone" and continues to be pulsatile. Scale bar $=10 \mu \mathrm{m}$.

\section{Supplementary video 5: FAK inhibitor treated, U2OS cells fail to respond to PDGF as} reported through FynSensor.

Live cell imaging of U2OS cells transfected with FynSensor was treated with FAK inhibitor and then stimulated with PDGF. Panel shows FRET index image using Fire LUT (Fiji) and scaled for ease of visualization. Individual images were acquired at $60 \mathrm{X}$ at an interval of $60 \mathrm{~s}$ and then stitched together to make a video (played at5 frames/second). Time stamp indicates elapsed time, "+FAKi" label shows the point at which FAK inhibitor was added and "+PDGF" indicates the point at which PDGF was added to the cells. Colour bar shows intensity of FRET signal. Localized activity of Fyn is greatly attenuated after treatment with FAK inhibitor. These cells also fail show any increase in FRET response after stimulation by PDGF. Scale bar= $10 \mu \mathrm{m}$. 


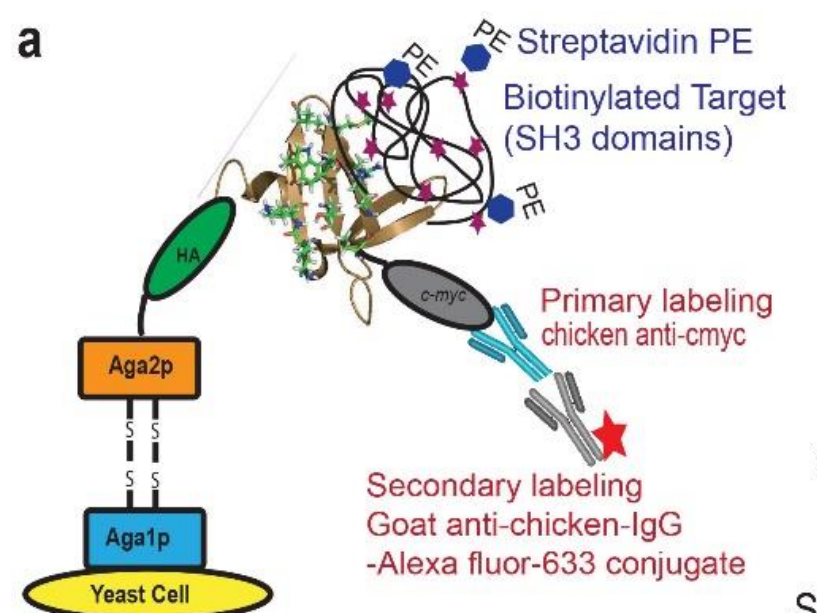

b Library consolidation \& growth
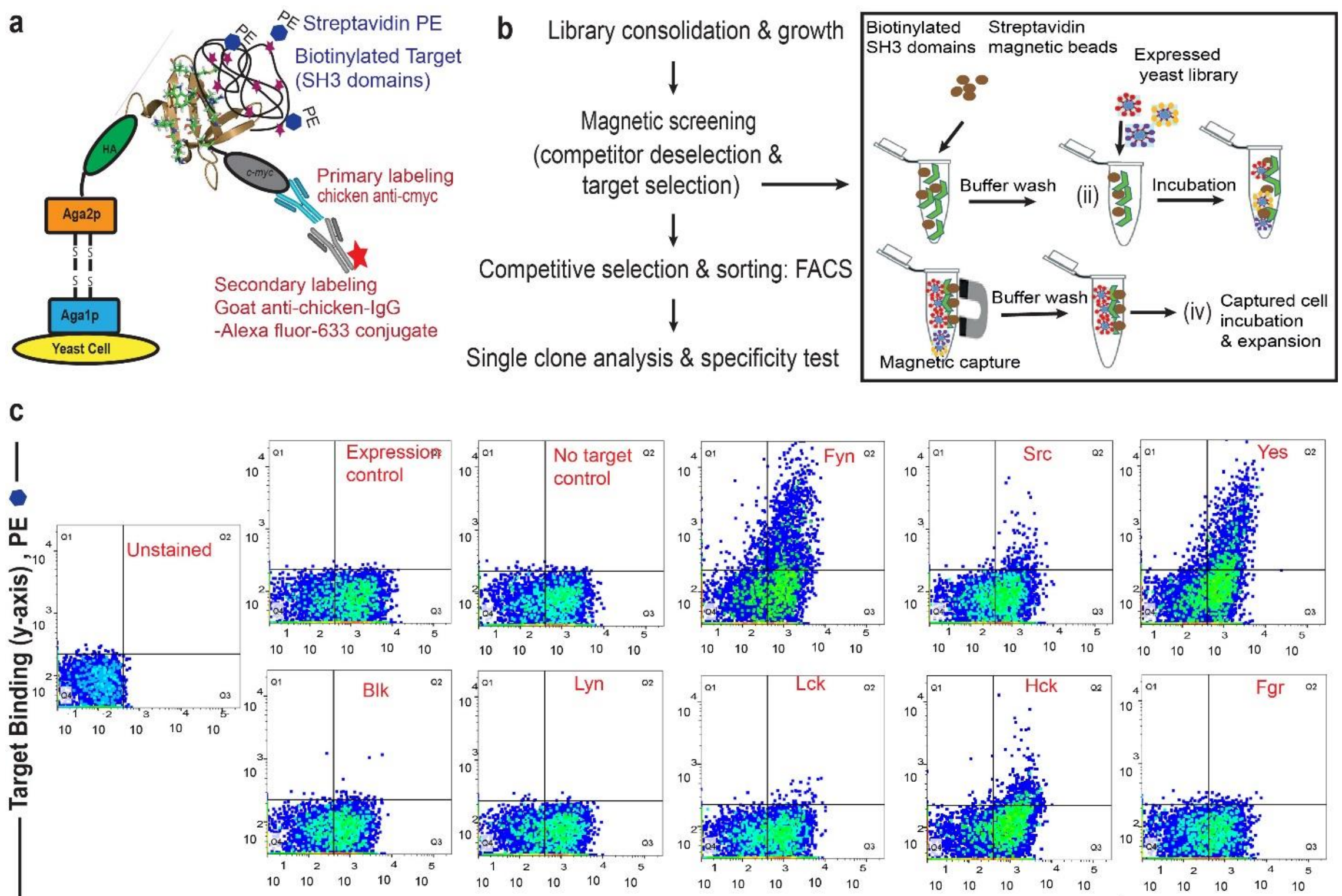

Magnetic screening

(competitor deselection \&

target selection)

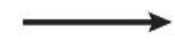

Competitive selection \& sorting: FACS $\downarrow$

Single clone analysis \& specificity test

Magnetic capture
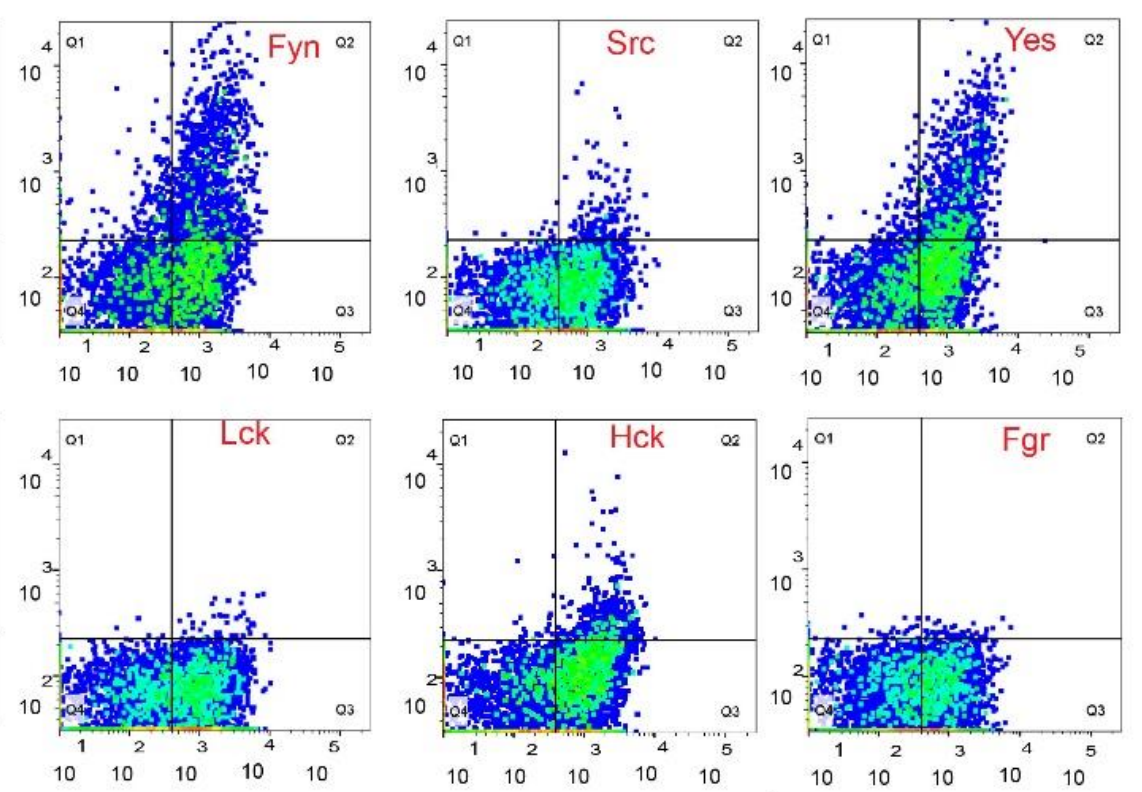

Library scaffold expression (x-axis), Alexa fluor-633 
80 Supplementary Fig. 1: Combinatorial library screening for a Fyn specific binder using yeast surface display of Sso7d variants.

(a) Schematic of yeast surface display platform. Briefly, a combinatorial library based on Sso7d scaffold (obtained by random mutagenesis of surface residues; Methods, 2013, 60, 15-26) was cloned in a yeast display vector. In this vector, Sso7d variants are flanked by HA tag and cmyc tags. Upon galactose induction, the Sso7d scaffold is expressed on the yeast surface as a fusion complex of Aga1p and Aga2p proteins along with HA and c-myc tags making it accessible for interaction with biotinylated target. The interaction of target and scaffold can be detected using immunofluorescence with Strep-PE (to detect biotinylated target) and a secondary antibody conjugated Alexa-Fluor 633 (for anti-c-myc antibody). The Alexa-Fluor 633 fluorescence corresponds to the full-length scaffold being expressed on the yeast cell surface, while the PE fluorescence intensity corresponds to the target SH3 domain presence. These features enable fluorescence assisted flow cytometer-based sorting of heterogeneous yeast cells (displaying variants of Sso7d scaffold) sensitive to target binding events. The figure is based on earlier reports (Methods in Molecular Biology, 2015, 1319, 155-175; Methods, 2013, 60, 15-26) (b) Work flow for binder screening (see Supplementary information for details). (c) FACS dot plot showing quantitative analysis of a pool of yeast cells obtained after magnetic selection and multiple rounds of FACS by flow cytometry to assess binding SH3 domains $(1 \mu \mathrm{M})$ from Src family of kinases. Data show binding of yeast clones to Fyn. Binding of some yeast cells is also detected for Yes and Hck; this is clarified further through single clone analysis (see Fig. 1 and Supplementary Fig. 2). 'Expression control' refers to yeast cells labelled with chicken anti-c-myc primary antibody and goat anti-chicken-IgG-Alexa Fluor 633 conjugate as secondary antibody. This control (antibody staining and subsequent FACS) confirms expression of the fusion protein containing Sso7d scaffold with a c-myc tag for detection. 'No target control' has been used to determine the specificity of Streptavidin-PE being used to label the target (i.e. biotinylated SH3 domains). 
bioRxiv preprint doi: https://doi.org/10.1101/726133; this version posted August 5, 2019. The copyright holder for this preprint (which was not certified by peer review) is the author/funder, who has granted bioRxiv a license to display the preprint in perpetuity. It is made available under aCC-BY-NC-ND 4.0 International license.

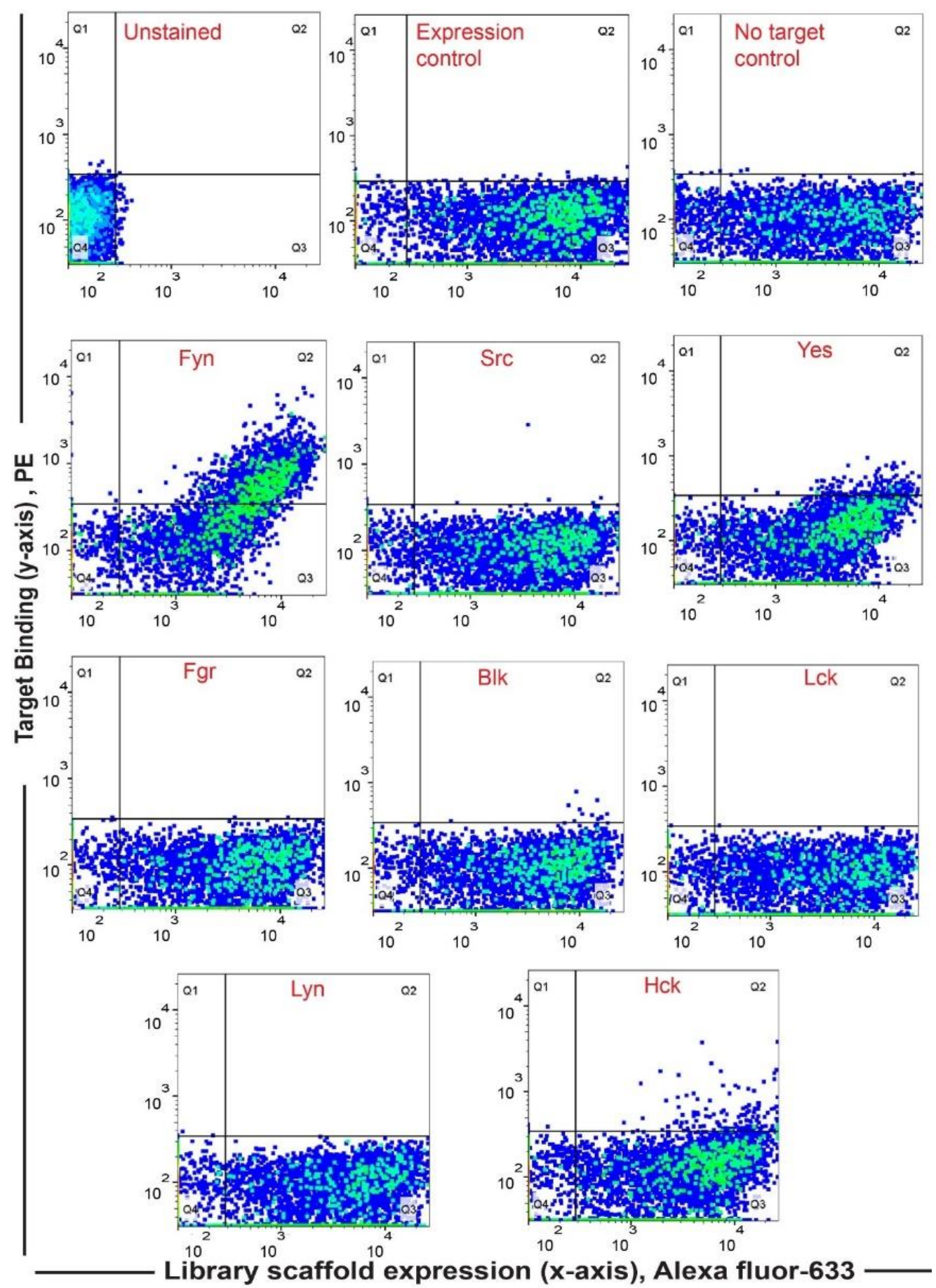

107 Supplementary Fig. 2: Screening yields new binder F29 that is highly specific for Fyn SH3.

Specificity analysis of the yeast clone 'F29' using FACS against SH3 domains of all the Src family of kinases $(1 \mu \mathrm{M})$ shows F29 preferentially binds Fyn. In all the graphs, fluorescence signal due to biotin-labeled target SH3 (Streptavidin-PE) is plotted against signal due to yeast cells expressing the c-myc-tagged Sso7d scaffold (Alexa 633 labelled anti-c-myc). Here we have scored for positive events wherein yeast cells likely expressing F29 are also stained for target with Strep-PE (for detailed explanation see Methods, 2013, 60, 15-26. Also see Supplementary Fig. 1). Expression and No-target controls were used as above. 


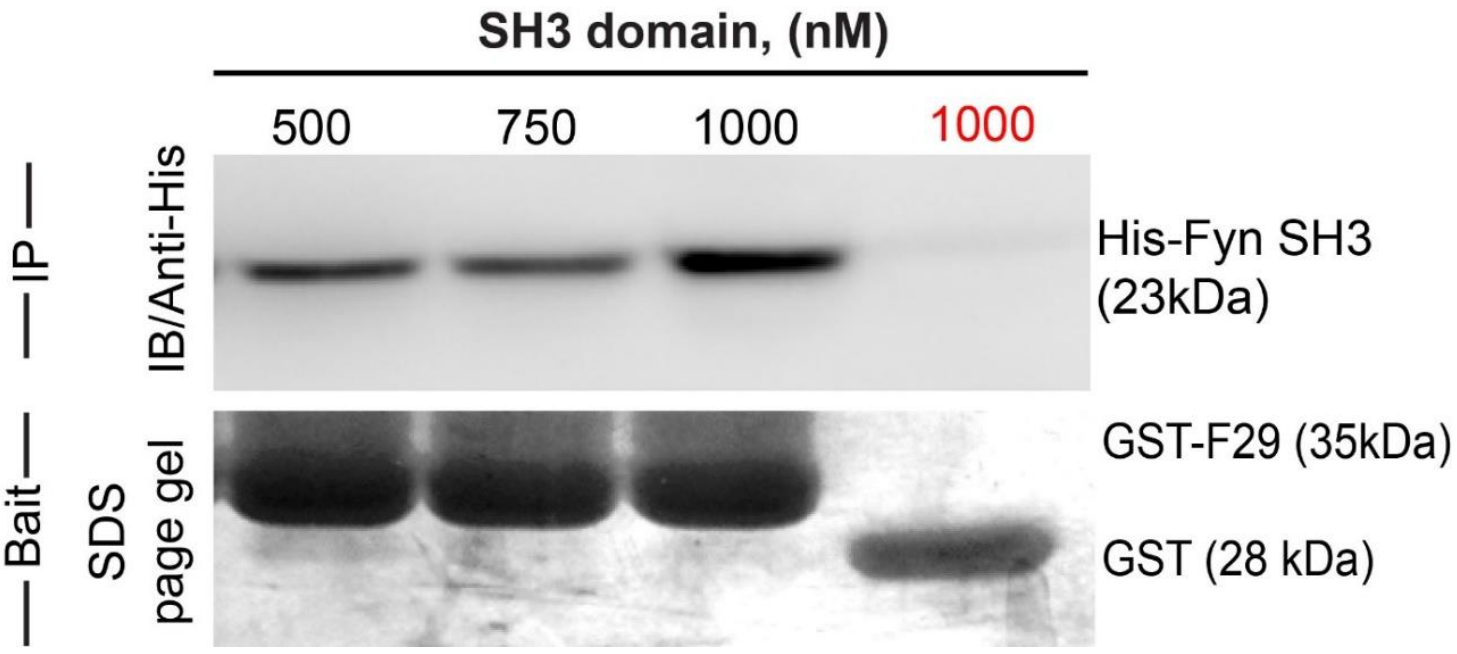

117 Supplementary Fig. 3: Newly identified, isolated protein binder F29 binds Fyn SH3

118 domain.

119 Binder F29 is able to recognize Fyn SH3 outside the context of the yeast surface, with 120 recombinantly expressed and purified F29 showing binding to Fyn SH3. Immunoblot showing 121 pull-down of His-tagged Fyn SH3 domains (500nM to 1000nM) using GST-F29 fusion protein, 122 immobilized as the bait on glutathione sepharose beads. In contrast GST-alone control beads 123 (GST protein immobilized glutathione sepharose beads) incubated with $1000 \mathrm{nM}$ of Fyn SH3 124 showed no pull-down (in red, last lane on the right). The His-tagged protein detected via anti125 6X-His antibody. Coomassie stained gel shows the bait protein bands immobilized on the beads 126 (GST-F29 or GST). 
bioRxiv preprint doi: https://doi.org/10.1101/726133; this version posted August 5, 2019. The copyright holder for this preprint (which was not certified by peer review) is the author/funder, who has granted bioRxiv a license to display the preprint in perpetuity. It is made available under aCC-BY-NC-ND 4.0 International license.

a

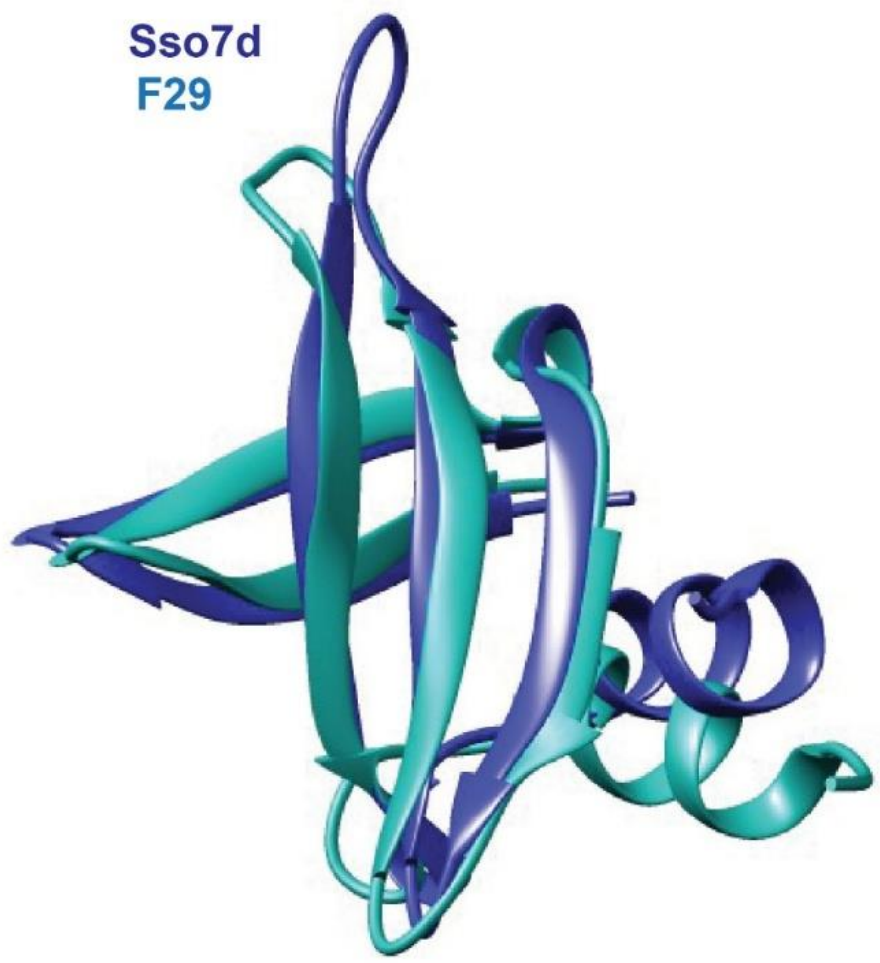

b

\section{Fyn-SH3}

F29

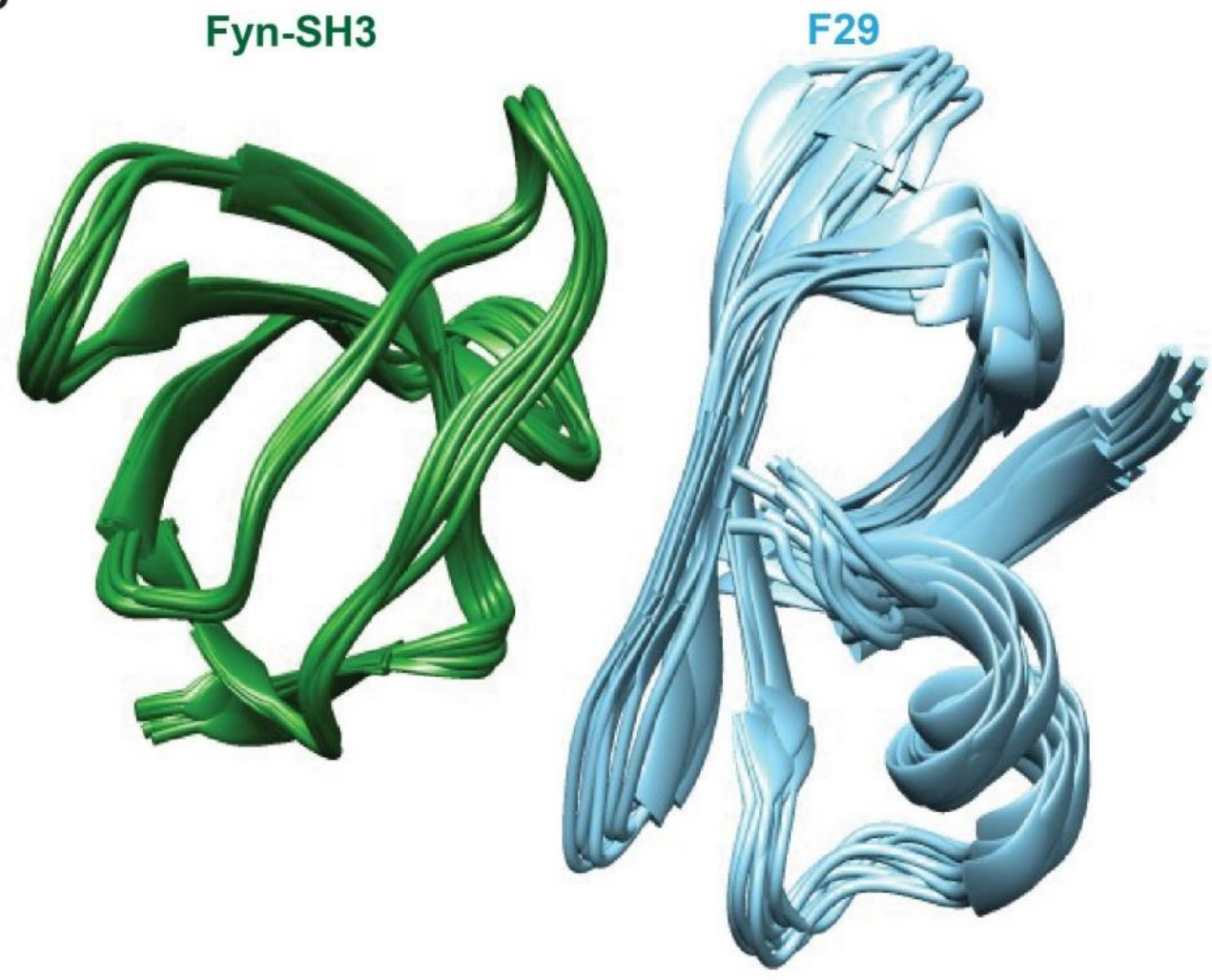
domain.

A superposition of (a) the CS-rosetta generated structure of F29 with Sso7d crystal structure. angstroms.(b) Ten lowest energy structures of Fyn-SH3:F29 complex generated by Haddock. 
a

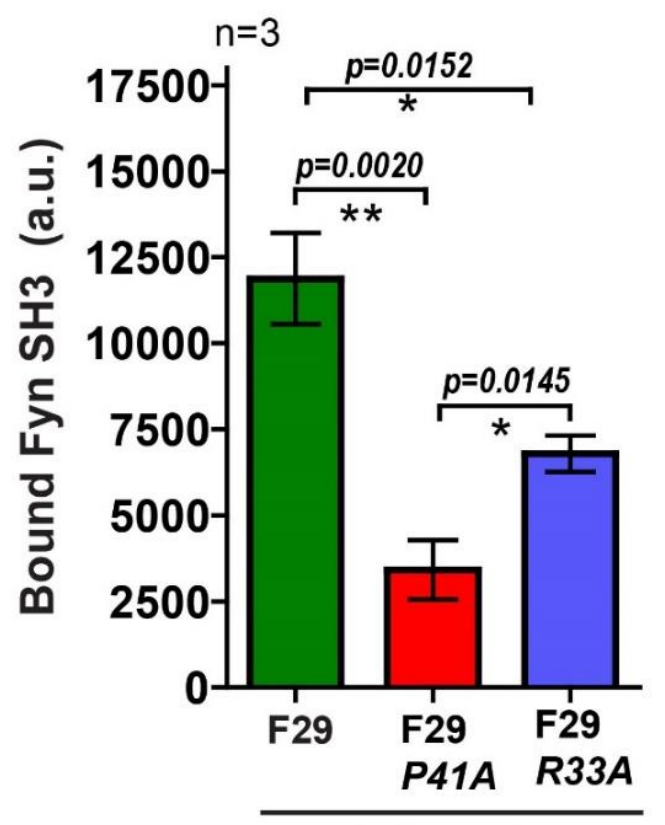

GST-F29

b

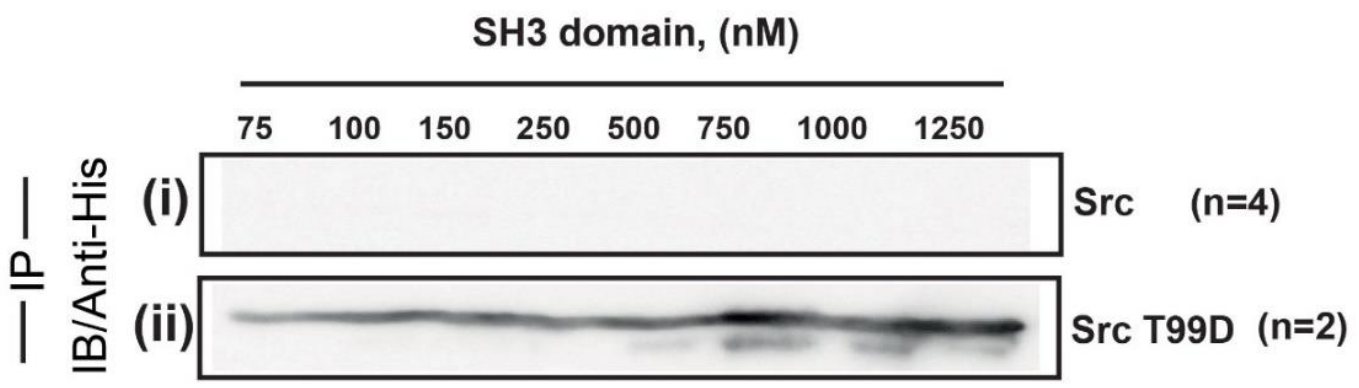

C

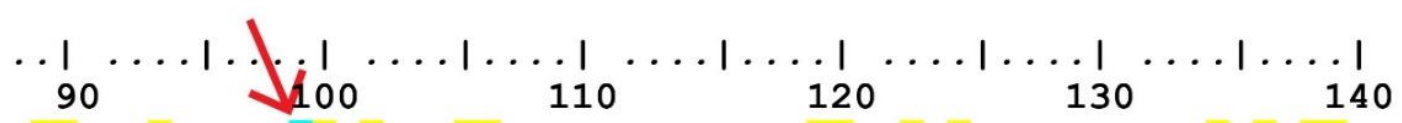

Fyn VAL YDYEARTEDD LSFHKGEKFQ ILNSSEGDWW EARSLTTGET GYIPSNYVAP

SrC VAL YDYESRTETD LSFKKGERLQ IVNNTEGDWW LAHSLSTGQT GYIPSNYVAP

Yes VAL YDYEARTTED LSFKKGERFQ IINNTEGDWW EARSIATGKN GYIPSNYVAP

Blk VAL YDYTAMNDRD LQMLKGEKLQ VLKGT-GDWW LARSLVTGRE GYVPSNFVAR

Lyn VAL YPYDGIHPDD LSFKKGEKMK VLEEH-GEWW KAKSLLTKKE GFIPSNYVAK

Lck IAL HSYEPSHDGD LGFEKGEQLR ILEQS-GEWW KAQSLTTGQE GFIPFNFVAK

Hck VAL YDYEAIHHED LSFQKGDQMV VLEES-GEWW KARSLATRKE GYIPSNYVAR

Fgr IAL YDYEARTEDD LTFTKGEKFH ILNNTEGDWW EARSLSSGKT GCIPSNYVAP

134 Supplementary Fig. 5: F29 specifically binds Fyn SH3 independent of the yeast cell surface and binding specificity can be switched through targeted mutagenesis.

Pull-down with F29 binder shows specific binding to Fyn SH3 domain. (a) Plot showing the amounts (band intensity, calculated from densitometry performed on immunoblot bands after pull-down) of bound Fyn SH3 domain to either immobilized GST-tagged binder F29 (by pulldown experiment) or F29 mutants P41A and R33A, respectively. Mutations, especially P41A attenuates binding significantly. Data from three independent experiments $(n=3)$ were analysed 
bioRxiv preprint doi: https://doi.org/10.1101/726133; this version posted August 5, 2019. The copyright holder for this preprint (which was

not certified by peer review) is the author/funder, who has granted bioRxiv a license to display the preprint in perpetuity. It is made available under aCC-BY-NC-ND 4.0 International license.

141 on GraphPad Prism. Student's unpaired one tailed-t-test was used to determine the p-value

142 (shown in the figure). Values are mean \pm s.d. (b) Immunoblot (probes with anti-6X-His 143 antibody) showing pull down of 6X-His tagged (i) Src SH3 domain and, (ii) Src SH3-T99D

144 domain mutant using GST-F29. While c-Src SH3 domain shows no binding, detectable binding 145 to F29 is observed for Src SH3 T99D domain mutant (c) Amino acid ClustalW alignment of 146 Src family member's SH3 domains. The residue numbers are according to full-length tyrosine147 protein kinase Fyn [Homo sapiens, (protein id-XP_016866143.1)]. The residues that are found 148 to be interacting with binder F29 (Fig. 3c) are also found to be conserved in these domains and 149 are highlighted. The Fyn SH3 residue (D99) that shows exclusive interaction with binder (R33) 150 is marked with an arrow. Introducing D99 into Src SH3 leads to measurable binding to F29 151 whereas WT Src SH3 domain shows no measurable interaction with F29 under identical 152 conditions. 
a

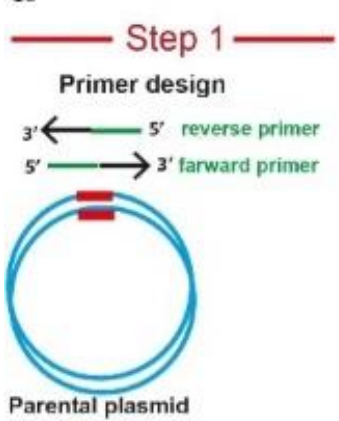

b
Step 2

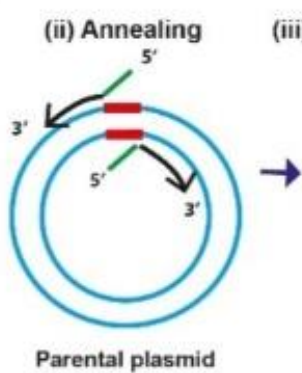

Parental plasmid

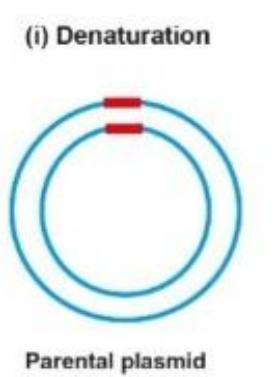

Parental plasmid

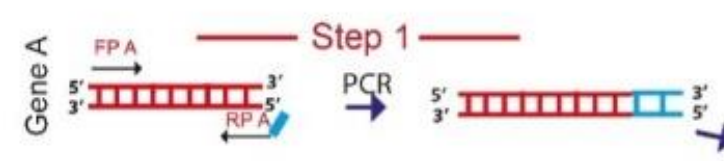

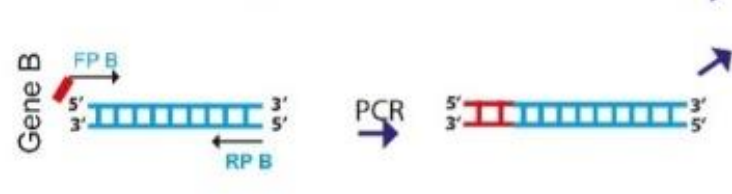

Used as

templates

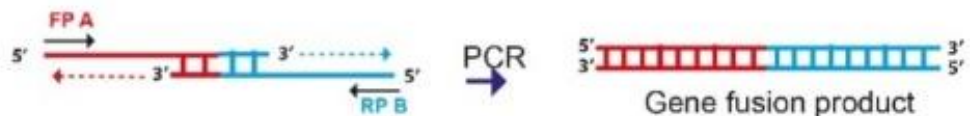

C
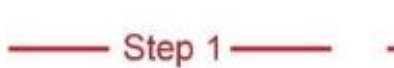

(i) Denaturation Step 2
Insert DNA preparation by
3. IIIIII'
$\overrightarrow{\mathrm{FP}} \downarrow$

3.

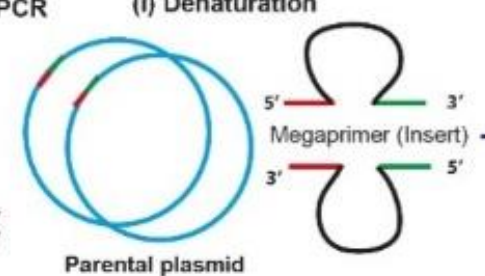

$\begin{array}{ll}\text { (ii) Annealing (iii) Extension } & \end{array}$

-Step $3-\quad-$ Step $4-$

Parental plasmid elimination Transformation by Dpnl digestion
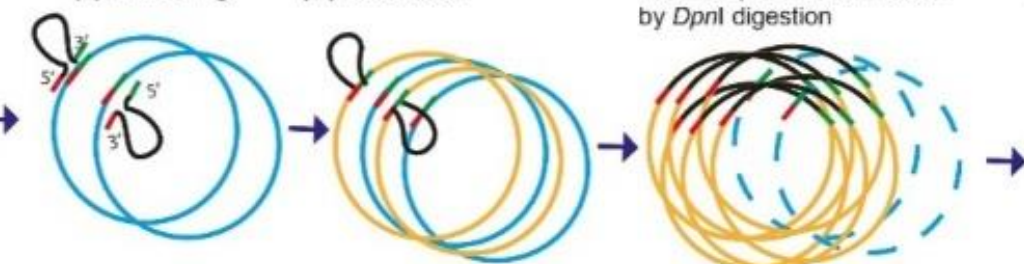
bioRxiv preprint doi: https://doi.org/10.1101/726133; this version posted August 5, 2019. The copyright holder for this preprint (which was

not certified by peer review) is the author/funder, who has granted bioRxiv a license to display the preprint in perpetuity. It is made available under aCC-BY-NC-ND 4.0 International license.

Supplementary Fig. 6: Step-wise representation of PCR based cloning methods for biosensor generation.

(a) 'Quick change' mutagenesis - The 'Quick change' mutagenesis method was adapted and used to perform point mutations, replace amino acids, deletion or insertion of single or multiple adjacent amino acids in a single tube reaction. This mutagenesis method was performed using Phusion high-fidelity DNA Polymerase (NEB) for mutagenic-primer directed replication of both parental plasmid strands. The primers, each complementary to opposite strands of the vector, were extended during temperature cycling by DNA polymerase, without primer displacement. Extended primers typically generated a mutated plasmid containing staggered nicks. Followed by temperature cycling, the products were treated with $D p n I$ restriction endonuclease (NEB) to digest methylated and hemi-methylated parental DNA. The mutationcontaining synthesized DNA products here selected as clones after transformation in E. coli. The method was adapted and modified similar to as described earlier (Methods in Enzymology, 1987, 154, 367-382; Nucleic acids research, 2004, 32, e115). (b) Overlapping PCR - In order to join two genes or DNA fragments A and B by PCR, the pair of primers FPA-RPA \& FPB \& RPB were designed. The primers RPA and FP B typically had overlapping regions with each other. The genes A and B were amplified and gel eluted from given template using these primers. In the next PCR cycle, these eluted PCR A and B products were taken (equimolar DNA) as template along with FPA and RPB primers and amplification performed. (c) Overlap extension PCR- This method was used to clone an insert into a plasmid without restriction endonucleases or T4 DNA ligase treatment. The method was similar to 'Quick change' mutagenesis, except here we used PCR amplified inserts of interest as large primers. The inserts needed to have the overlap region of plasmid site for cloning. The strategy and schematics were adapted from an earlier report (Bio-Techniques, 2010, 48, 463-465). 
bioRxiv preprint doi: https://doi.org/10.1101/726133; this version posted August 5, 2019. The copyright holder for this preprint (which was

not certified by peer review) is the author/funder, who has granted bioRxiv a license to display the preprint in perpetuity. It is made available under aCC-BY-NC-ND 4.0 International license.

a

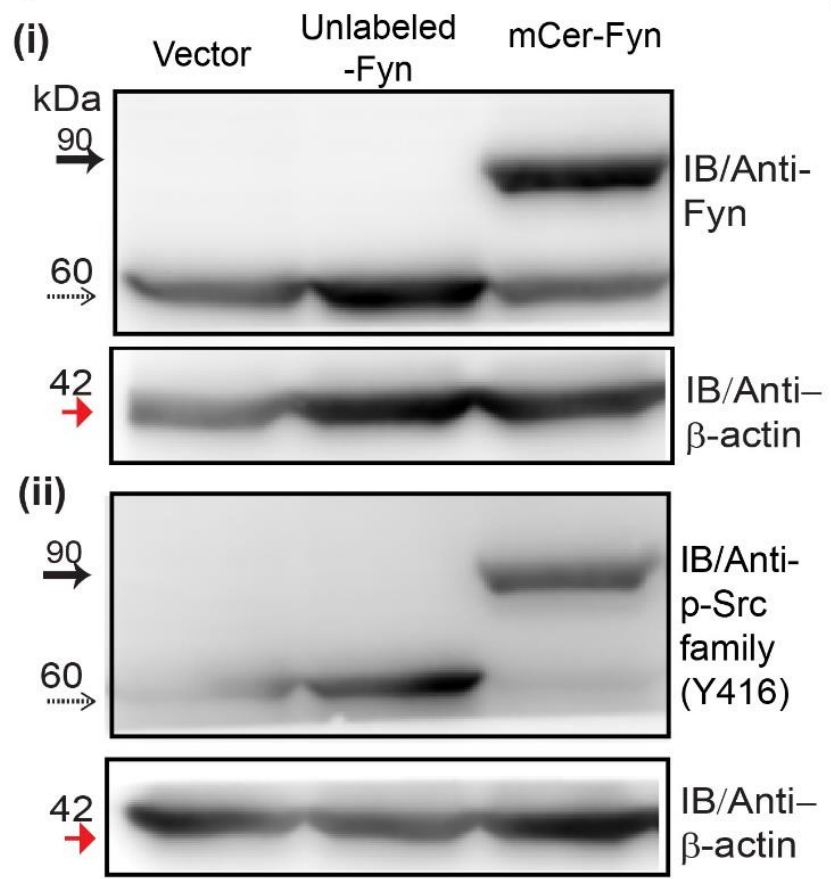

b

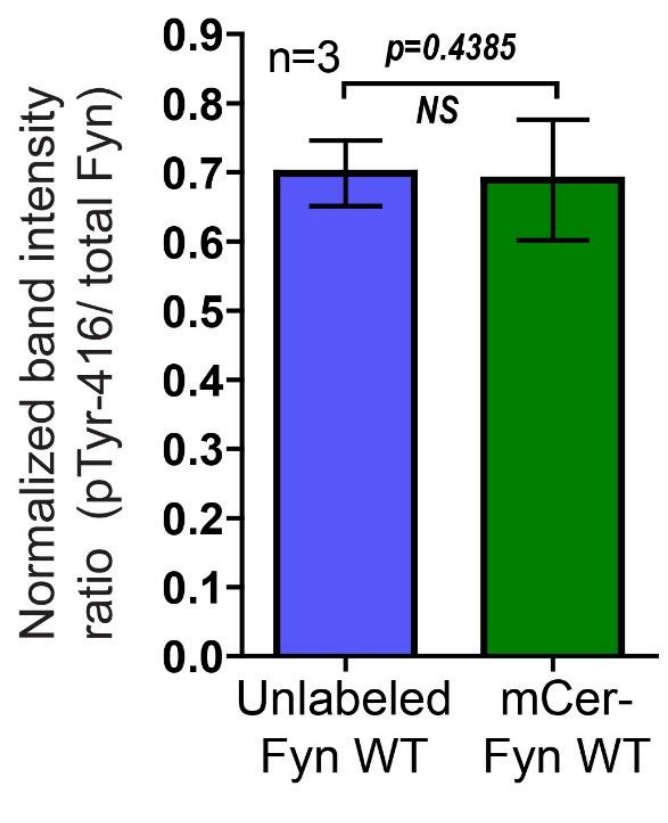

C

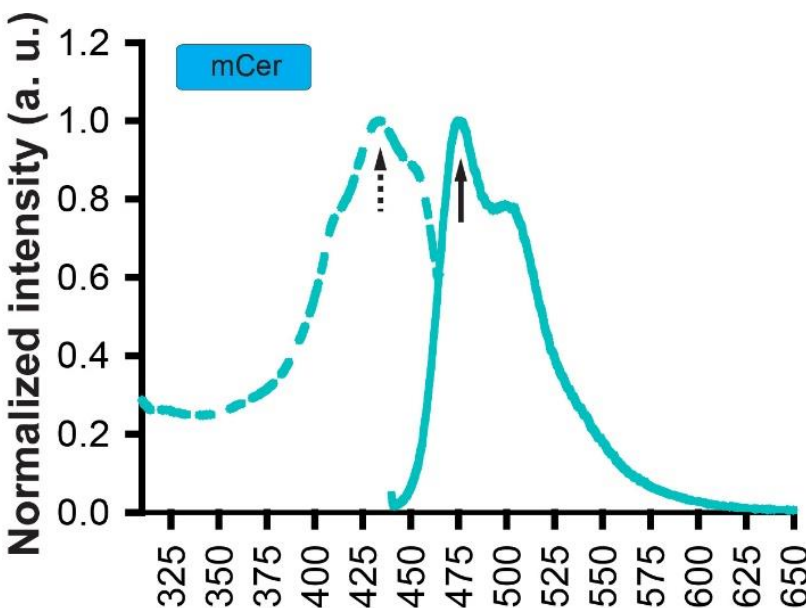

Wavelength $(\mathrm{nm})$

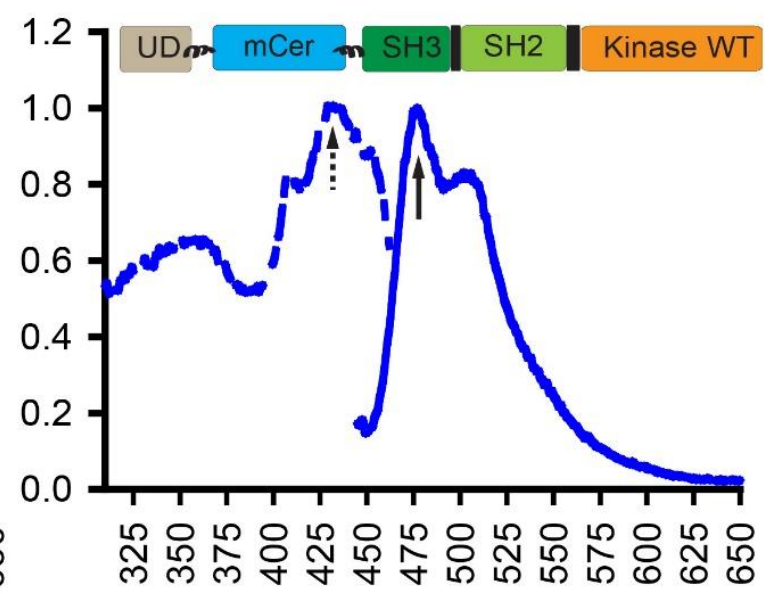

Wavelength $(\mathrm{nm})$

Supplementary Fig. 7: Labeled Fyn biosensor, mCer-Fyn is active, fluorescent, resistant to proteolysis and shows activity (autophosphorylation) levels identical to unlabeled, wild-type kinase.

(a) Immuno-blot of FRET donor (mCer-Fyn) and unlabeled Fyn construct ectopically expressed in HEK-293T cells. The lysate $(50 \mu \mathrm{g})$ was resolved on $8 \%$ SDS gel followed by PVDF membrane transfer and probed using (i) anti-Fyn antibody, as well as (ii) anti-phosphoSrc family (Tyr416) antibody. Also shown is a loading control, $\beta$-actin ( 42 kDa, red arrow). In each panel, the labeled kinase (with mCerulean insertion, mCer-Fyn) bands ( $\sim 90 \mathrm{kDa})$ are shown with solid arrows while the unmodified or unlabeled Fyn kinase variants $(\sim 60 \mathrm{kDa})$ are shown with dotted arrows. (b) Kinase auto-phosphorylation levels observed with FRET donor 
bioRxiv preprint doi: https://doi.org/10.1101/726133; this version posted August 5, 2019. The copyright holder for this preprint (which was

not certified by peer review) is the author/funder, who has granted bioRxiv a license to display the preprint in perpetuity. It is made available under aCC-BY-NC-ND 4.0 International license.

190 labeled wild-type (wt) Fyn (mCer-Fyn) compared with autophosphorylation of the unlabeled, 191 WT Fyn kinase using immuno-blot analysis from panel a. In each case the band intensity was 192 normalized with their loading controls. The ratios of active Fyn (pTyr416) to total Fyn have 193 been plotted for both unmodified and FRET-donor (mCer-Fyn) from three such blots, $(n=3)$. 194 Data sets were compared using Student's unpaired one tailed-t-test on GraphPad Prism. The 195 extent of autophosphorylation for both labeled (mCer-Fyn) and unlabeled wild-type Fyn were 196 virtually identical ( $p$-value=0.4385). Values are mean \pm s.d. (c) Whole cell fluorescence 197 excitation (dotted line) and emission (solid line) spectra of HEK-293T cells ectopically 198 expressing either FRET donor construct (mCer-Fyn, wt) or unmodified mCerulean. For 199 emission spectra, $\lambda_{\mathrm{ex}}=435 \mathrm{~nm}$ and for excitation spectra, $\lambda_{\mathrm{em}}=475 \mathrm{~nm}$. Labeled Fyn (mCer200 Fyn) expressing cells show spectra similar to cells expressing untagged mCerulean. 
a

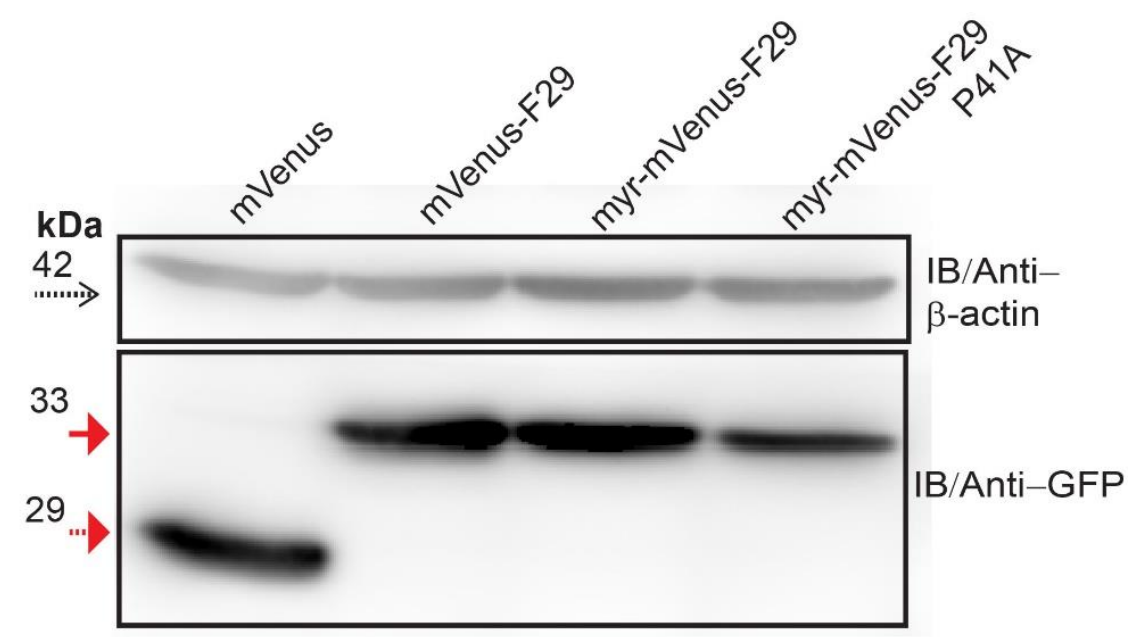

b
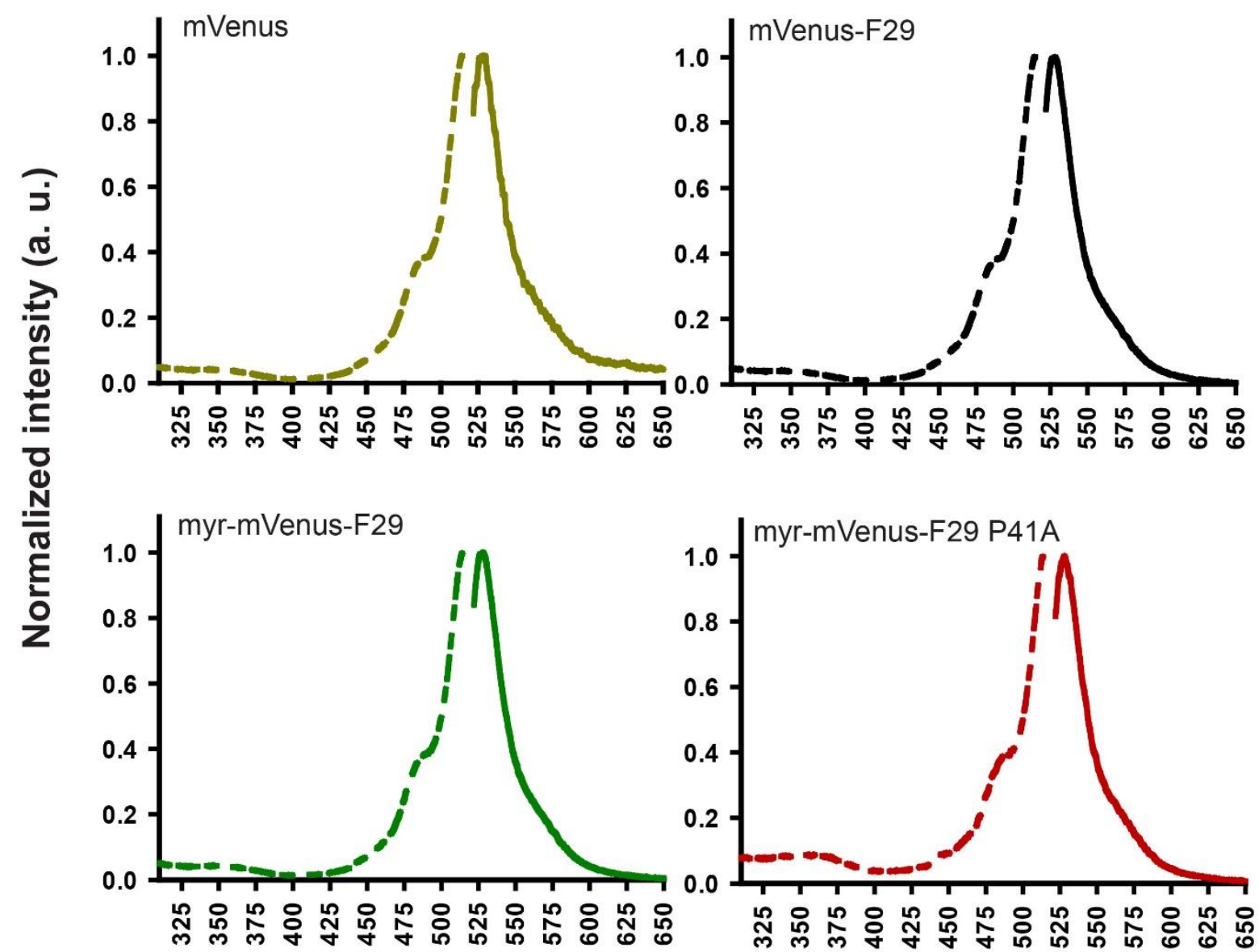

\section{Wavelength $(\mathrm{nm})$}

Supplementary Fig. 8: Labeled F29 expressed in cells is resistant to proteolysis and shows expected fluorescence in cells.

a) Immuno-blot analysis of FRET acceptor (mVenus-F29 and its variants) expressed in HEK293 T cells. $\beta$-actin serves as loading control (dotted arrow). mVenus (29kDa) alone or FRETacceptor variants $(\sim 35 \mathrm{kDa})$ probed with GFP antibody. (b) Whole cell fluorescence excitation 
bioRxiv preprint doi: https://doi.org/10.1101/726133; this version posted August 5, 2019. The copyright holder for this preprint (which was

not certified by peer review) is the author/funder, who has granted bioRxiv a license to display the preprint in perpetuity. It is made available under aCC-BY-NC-ND 4.0 International license.

207 (dotted line) and emission (solid line) spectral measurements of HEK-293T cells expressing 208 either labeled mVenus F29, mVenus F29 with a myristoyl tag (myr-mVenus-F29), mVenus209 myr-F29 P41A (non-binding control) or mVenus alone. In case of cells expressing each labeled 210 F29 variant, the fluorescence spectra were virtually identical to the spectra observed from cells 211 expressing untagged $\mathrm{mVenus}$ with $\lambda_{\mathrm{ex}}=515 \mathrm{~nm}$ and $\lambda_{\mathrm{em}}=525 \mathrm{~nm}$. 
a (i)

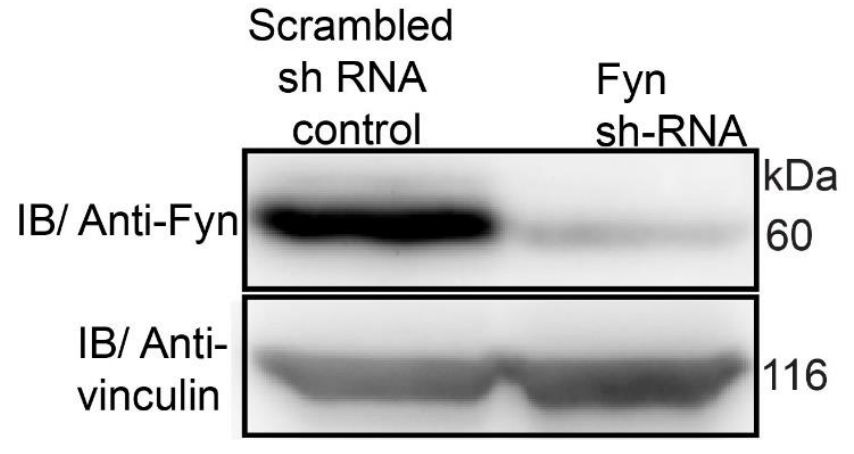

(ii)

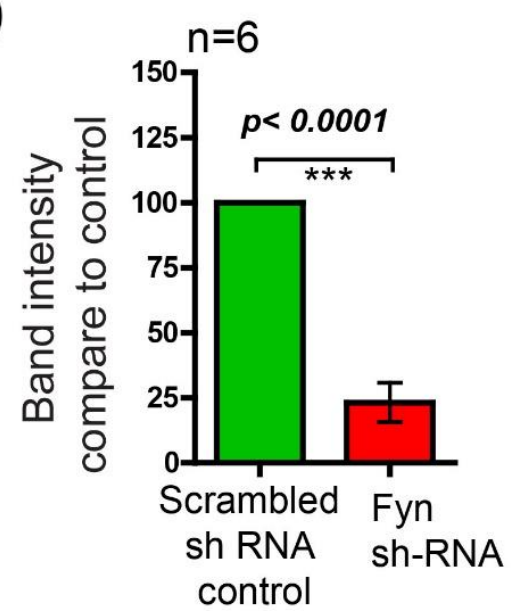

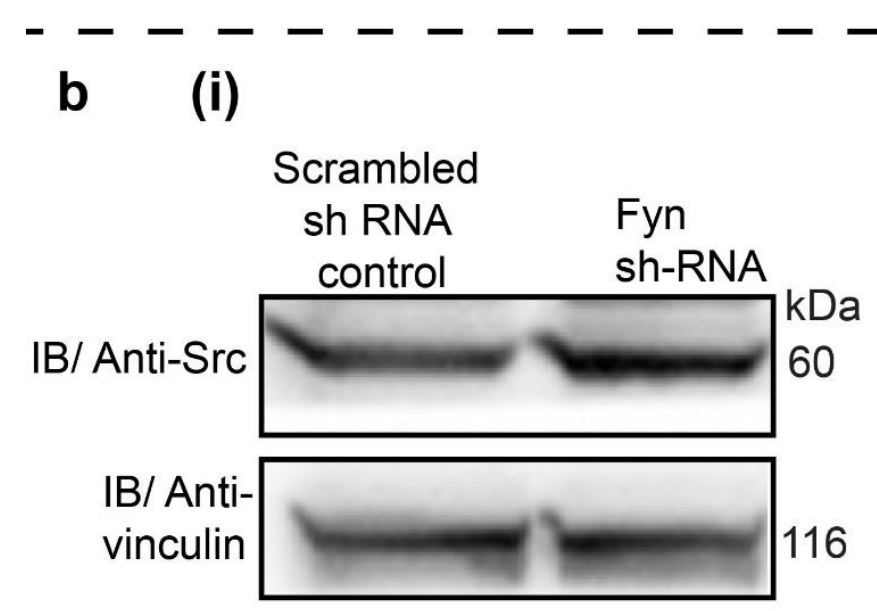

(ii)
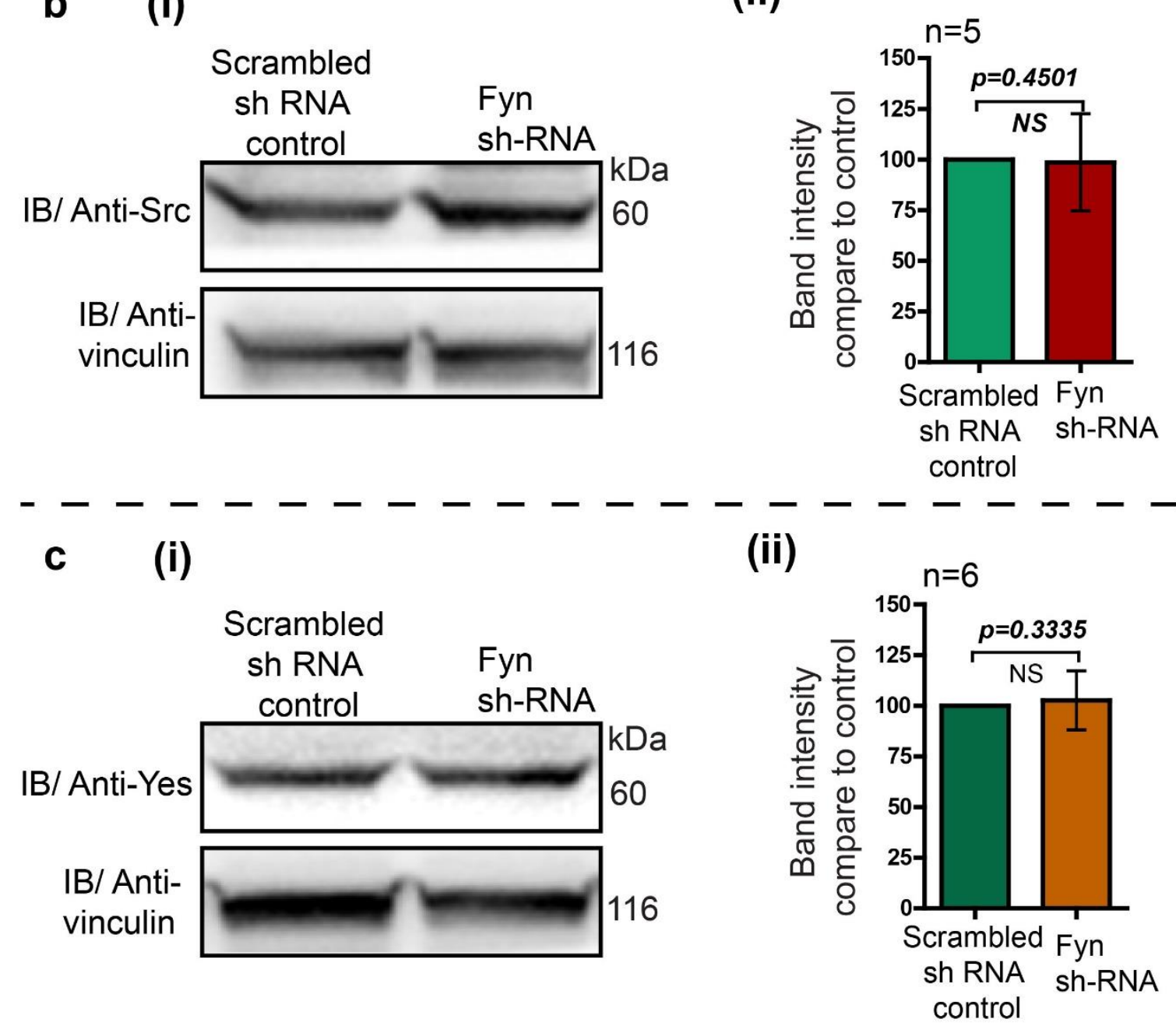

Supplementary Fig. 9: Knock-down of endogenous Fyn kinase expression using RNA interference in HEK293T cells.

215 RNA interference leads to a substantial reduction in Fyn levels while levels of c-Src and c-Yes are not affected. The HEK293T cells were transduced with retroviral construct having Fyn 3'UTR targeting Sh-RNA. The transduced cells were selected using puromycin. No target Sh- 
bioRxiv preprint doi: https://doi.org/10.1101/726133; this version posted August 5, 2019. The copyright holder for this preprint (which was

not certified by peer review) is the author/funder, who has granted bioRxiv a license to display the preprint in perpetuity. It is made available under aCC-BY-NC-ND 4.0 International license.

218 RNA (scrambled) was used as control (see Supplementary methods). The cells were grown and

219 cell lysate were analysed using immunoblot assay to measure the total levels of endogenous

220 Src family members (a) Fyn, (b) Src and, (c) Yes kinases using specific antibodies. In each of

221 the cases, (i) immunoblot were probed with specific antibodies (Src, Yes or Fyn) as well as

222 vinculin as loading control (ii) quantitative analysis of more than five such blots showing the

223 normalized expression level of respective kinases compared to scrambled sh-RNA control

224 cells. Fyn protein levels show a substantial reduction while levels of Src and c-Yes do not show

225 any significant change. The data was analysed using Student's unpaired one tailed-t-test on

226 Graph-pad Prism and corresponding $p$ values are shown on respective panels. Values are

227 mean \pm s.d. 


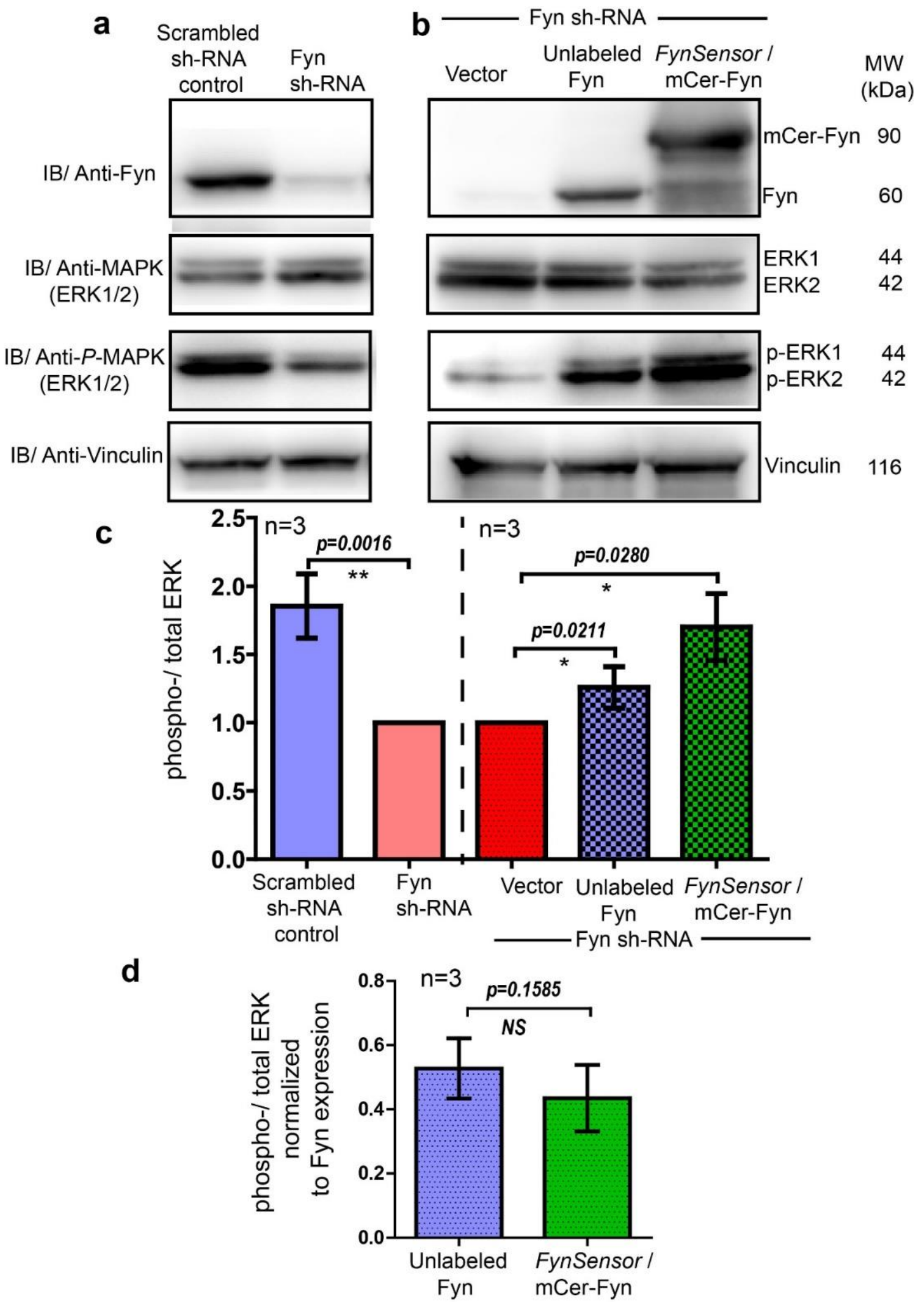

Supplementary Fig. 10: FynSensor (mCerulean labeled) Fyn is signaling competent and rescues ERK phosphorylation in Fyn knock-down cells similar to unlabeled Fyn.

a) Cellular Fyn levels regulate downstream signaling, as measured through levels of ERK phosphorylation. Immunoblots showing levels of Fyn kinase (60 kDa), total-ERK as well as 
phospho-ERK (42-44 kDa) in control (scrambled sh RNA control) as well as stable Fyn knockdown HEK293T cells (Fyn KD cells generated through RNAi). Reduction in cellular Fyn levels causes a reduction in extent of ERK phosphorylation. Vinculin (116 kDa) has been used as loading control. (b) FynSensor (mCerulean labeled) mCer-Fyn is able to rescue downstream signaling in Fyn-knockdown cells. Specifically, expression of mCer-Fyn in Fyn-knockdownHEK293T (FynKD) cells is able to reverse the reduction in ERK phosphorylation, that is otherwise observed in FynKD cells. This rescue is similar to that observed with unlabeled wildtype Fyn. Briefly, FynKD cells were transiently transfected with either vector control, unlabeled Fyn kinase (wt) or labeled FynSensor-Fyn (mCer-Fyn), and the extent of ERK phosphorylation probed. Immunoblot showing the levels of Fyn, total-ERK as well as phosphoERK. Vinculin was used as loading control. (c) Ectopic expression of FynSensor-Fyn in FynKD cells causes a significant enhancement in levels of ERK phosphorylation. Bar graph showing extent of ERK phosphorylation (ratio of phospho-/total-ERK) in RNAi control as well Fyn knockdown cells (left panel, also see a). There is a significant reduction in ERK phosphorylation (ratio of phospho-/total-ERK) in FynKD cells. Further, in this FynKD background, the recovery of ERK phosphorylation (ratio of phospho-/total-ERK) by expressing either labeled Fyn (mCer-Fyn) or unmodified/unlabeled, WT Fyn is quantified (right panel, also see $\boldsymbol{b}$ ). (d) Extent of ERK phosphorylation induced by ectopic Fyn rescue (in Fyn-knockdown cells) is similar for unlabeled and labelled (FynSensor) Fyn. Bar graph showing ratio of phospho-/total-ERK, when normalized for Fyn expression levels, for labeledFyn (FynSensor) as well as unlabeled-wt-Fyn transiently expressed in Fyn knockdown cells (as shown in b). No significant difference is observed between labeled and unlabeled kinase. For $\mathbf{c}$ and $\mathbf{d}$, immunoblot band intestines from three independent experiments each were analysed using Student's unpaired one tailed-t-test on Graph-pad Prism and corresponding $p$ values are shown on respective panels. Values are mean \pm s.d. 
a
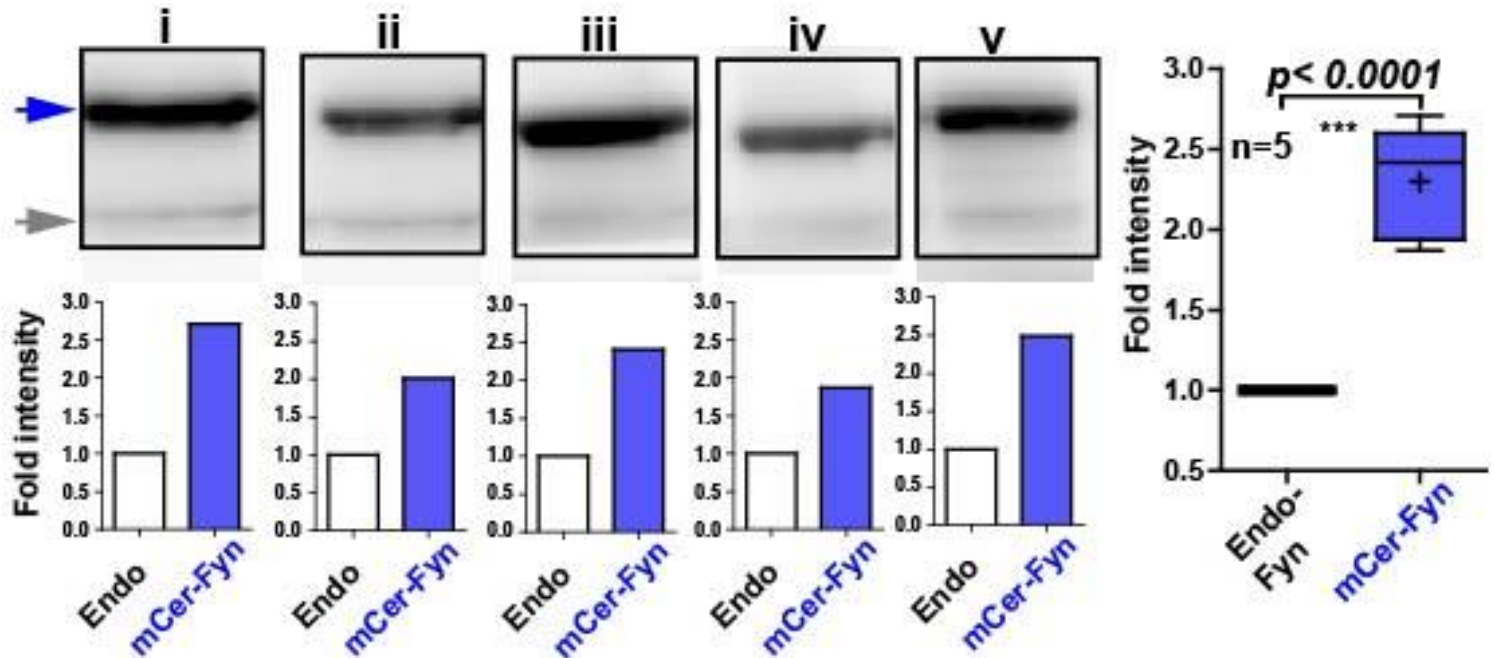

b

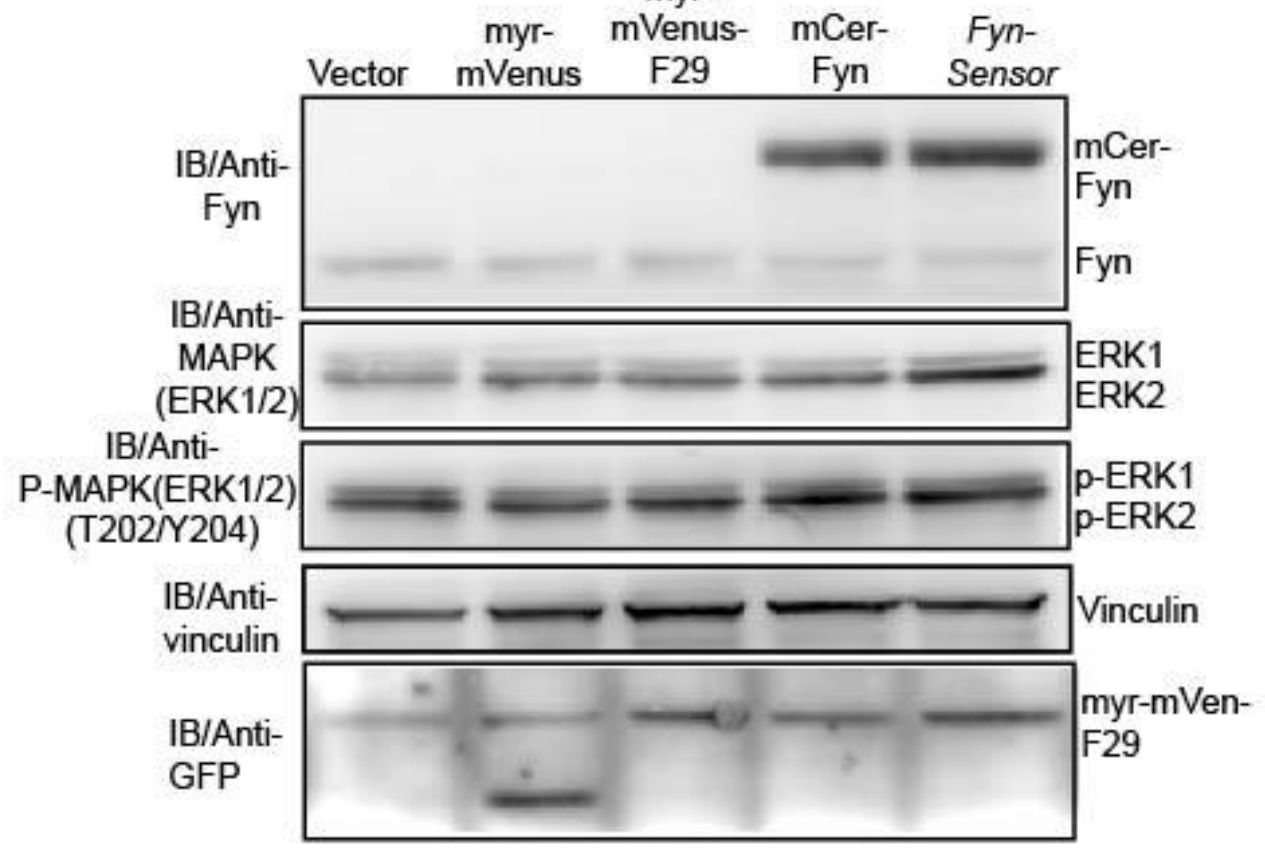

MW

$(\mathrm{kDa})$

C

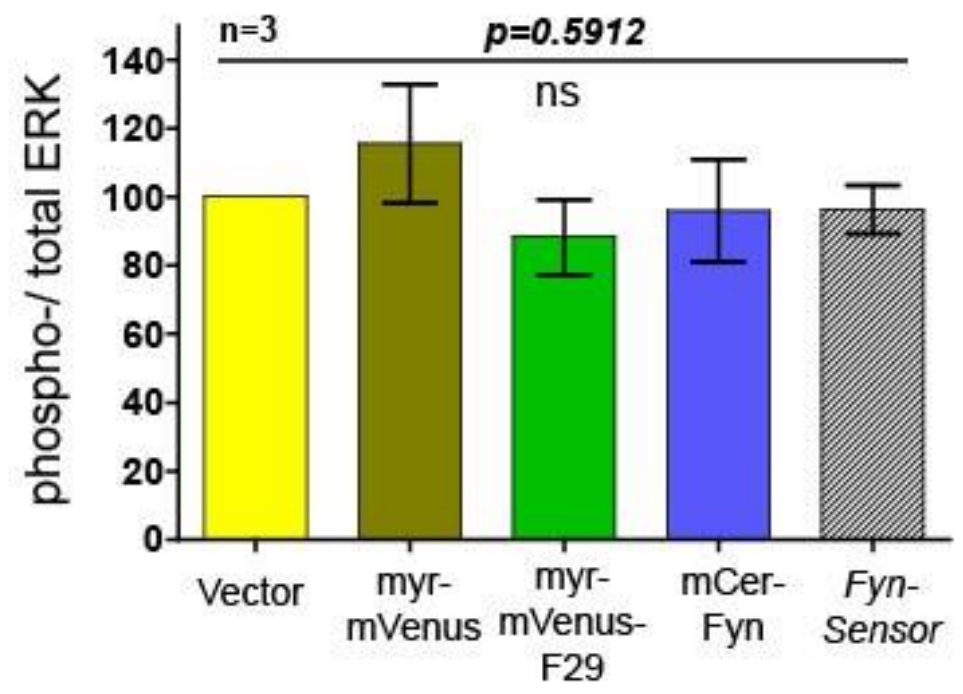

259 Supplementary Fig. 11: FynSensor, labelled Fyn and F29 binder do not perturb downstream signalling at the expression levels used for FynSensor imaging. 
Under expression levels used for biosensor imaging (quantified here in U2OS cells), the FynSensor labeled Fyn (mCer-Fyn) does not perturb downstream signalling as measured through ERK phosphorylation. (a) Immuno blot assay performed on cells transiently transfected with the FynSensor DNA constructs (same as used for biosensor imaging; donor: acceptor ratio, 1:2) and probed with Anti-Fyn antibody. Here five immunoblots (i-v) are shown where mCer-Fyn $(90 \mathrm{kDa})$ band has been marked with blue arrowhead and endogenous Fyn band is marked with grey arrowhead. For each case (i-v) the band intensity mCer-Fyn was compared with endogenous Fyn and plotted using GraphPad Prism and shown in a Box-andWhisker plot. Data from five blots (i-v) analysed using Student's unpaired one tailed t-test on GraphPad Prism (respective p values are indicated on panel). Average from FIVE independent experiments show that mCer-Fyn is expressed at levels LESS THAN 2.5 times that of the endogenous Fyn kinase. Imaging data in manuscript (see Fig. 6, 7c, 8a\& 9) show that Fyn activity patterns revealed by FynSensor remain the same, independent of mCer-Fyn expression in this current expression range.

Expression of neither FynSensor (mCer-Fyn + myr-mVenus-F29), labelled Fyn (mCer-Fyn) nor labelled F29 (myr-mVenus-F29) alone perturb downstream signalling in U2OS cells, as measured through ERK phosphorylation levels. (b) Immunoblot showing levels of phosphorylated (anti-p-ERK) and total ERK (anti-ERK) in U2OS cells expressing either FynSensor, labelled Fyn (mCer-Fyn), labelled F29 (myr-mVenus-F29), a myristoyl-taggedmVenus or a vector control. Also shown are levels of Fyn (anti-Fyn) and vinculin (antivinculin, control) as well as levels of myr-mVenus-F29 and myr-mVenus (anti-GFP).(c) Measurement of levels of ERK phosphorylation in cells expressing either FynSensor, labelled Fyn, labelled F29, a myristoyl-tagged-mVenus or a vector control. Graphs showing ratios of phospho-/total-ERK from immunoblot band densities from three independent experiments. No significant changes were seen in ERK phosphorylation levels. The pairs of means were tested using Newman-Keuls test in one-way ANOVA. The corresponding $p$ values are shown on respective panels. Values are mean \pm s.e.m.

Note: We do see a nonspecific band at $\sim 33 \mathrm{kDa}$ in lanes 1 and 2 in blots shown in Supplementary Fig. 11 likely due to non-specific binding of the anti-GFP antibody to some species present in U2OS cell lysates. However, in HEK293T cells and C2C12 cells, we do not observe any such corresponding 33kDa nonspecific band (See lane 1 of Supplementary Fig. 8 and lanes 1,2 and 4 of Supplementary Fig. 14). 


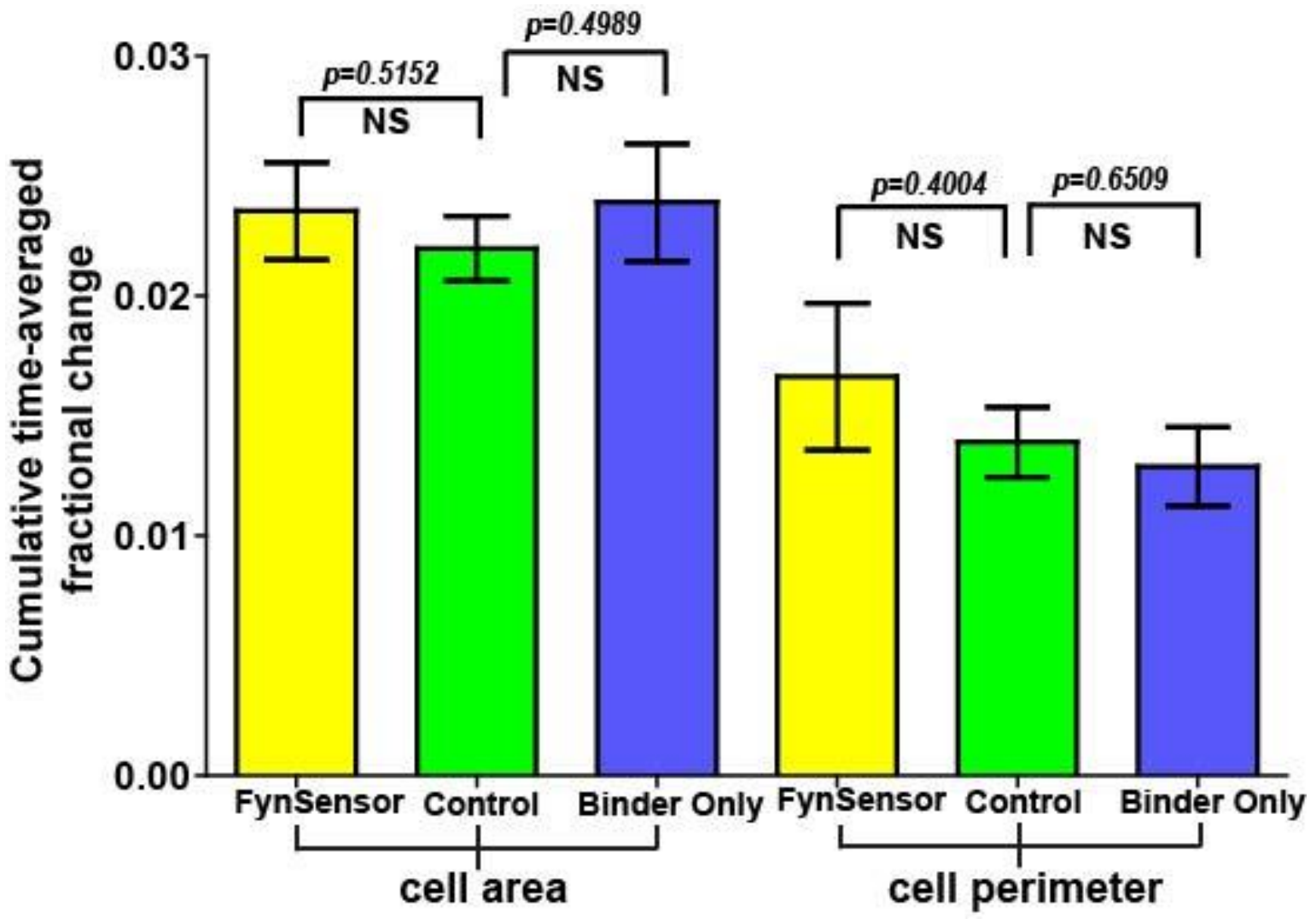

Supplementary Figure 12: Cellular expression of FynSensor or Fyn binder 'F29' does not perturb cellular morphodynamics.

297 U2OS cells transiently expressing either FynSensor (mCer-Fyn AND myristyol-tag-mVenusF29) or just myr-mVenus-F29 binder alone or a control fluorescent protein construct (myrmVenus) were imaged and two morphodynamic parameters, namely, cumulative timeaveraged change in cell area and cell perimeter were calculated through computer-aided quantitative image analysis. Details of the analysis are provided in the methods section. Under the imaging conditions used in this manuscript, the three image sets show no statistically significant differences, suggesting that the expression of FynSensor or the F29 binder has no measurable effect on cell morphodynamics. Values are mean \pm s.e.m. Student's two-tailed unpaired t-test has been used to determine the p-value. 
a TIRF micrograph

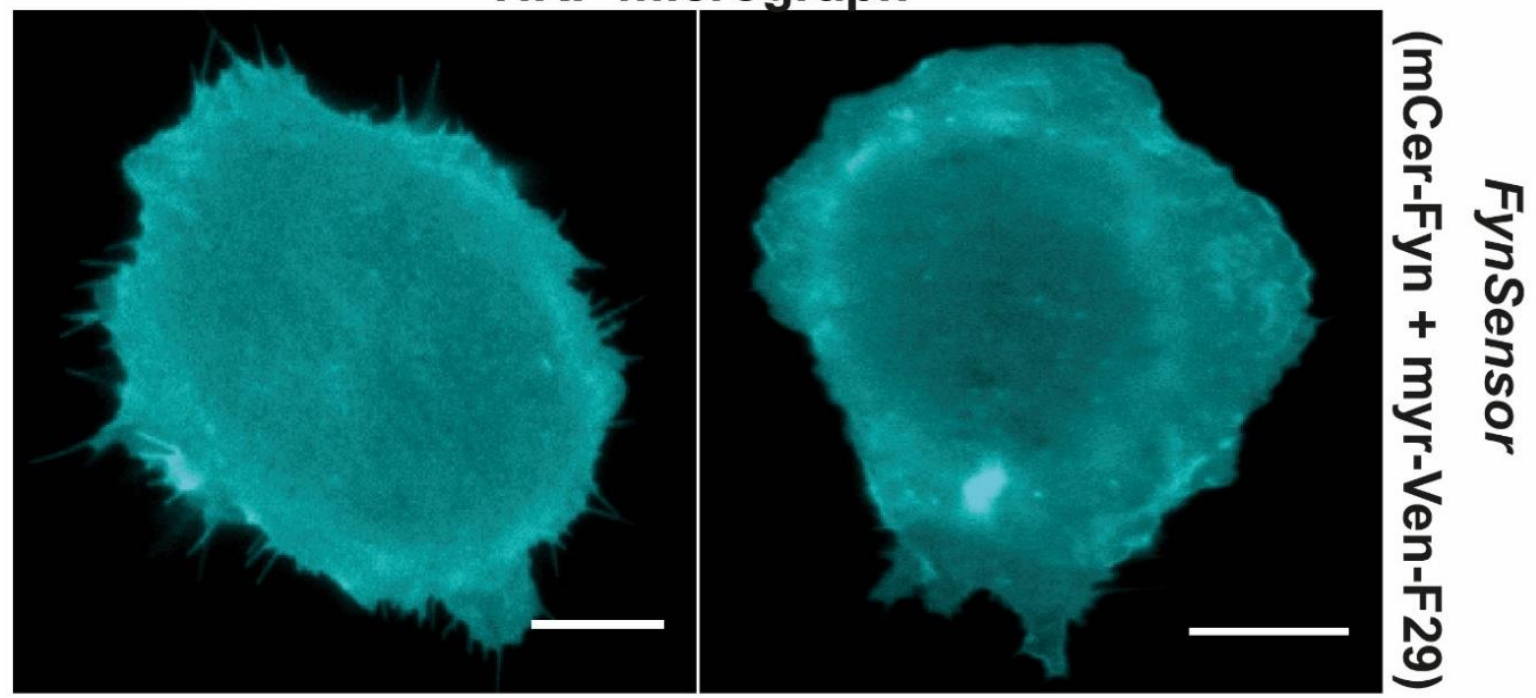

b

Confocal micrograph

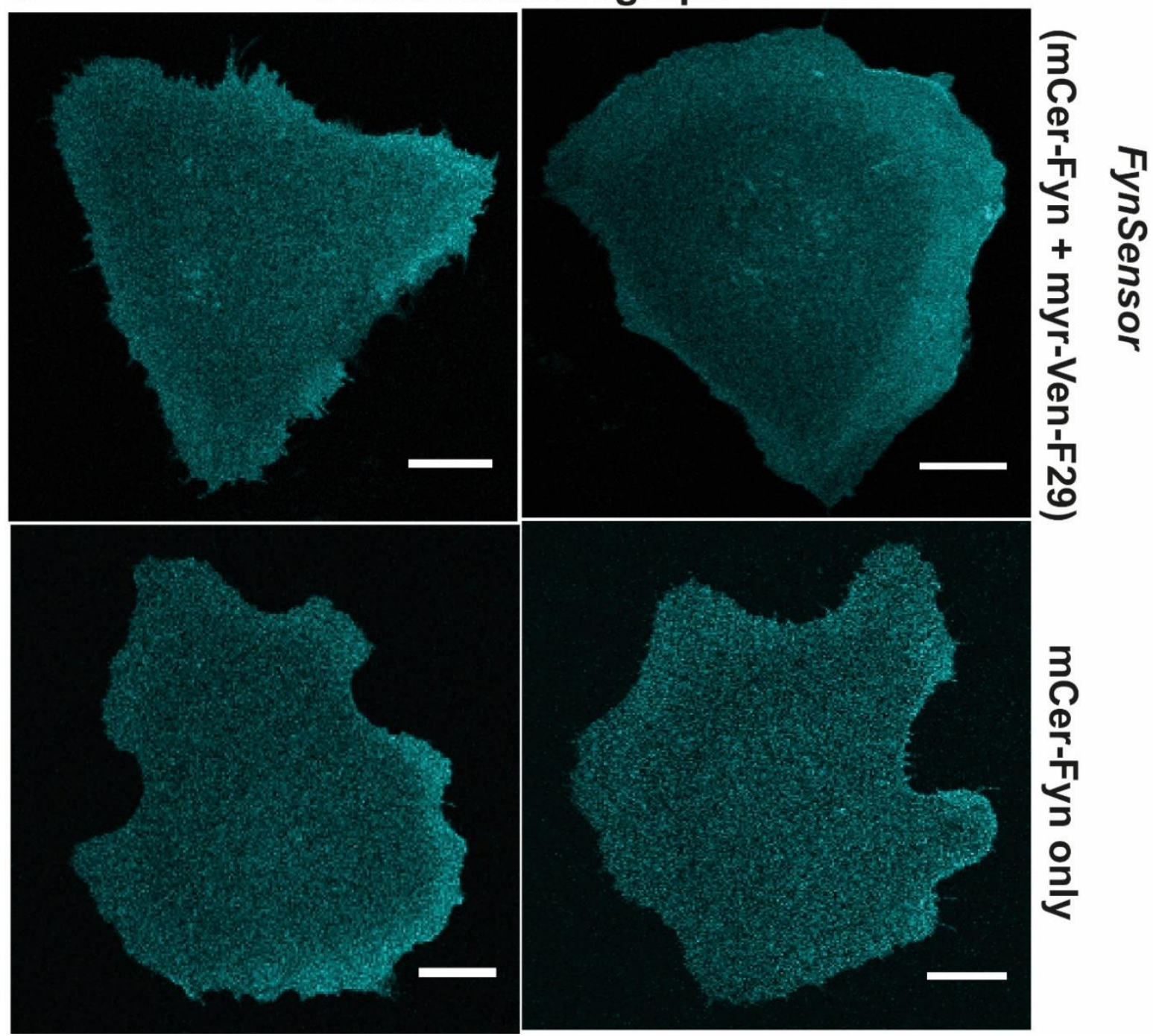

Supplementary Figure 13: Labeled FynSensor Fyn (mCer-Fyn) kinase localizes to the plasma membrane in cells and the localization is not affected by presence of F29 binder. 
bioRxiv preprint doi: https://doi.org/10.1101/726133; this version posted August 5, 2019. The copyright holder for this preprint (which was

not certified by peer review) is the author/funder, who has granted bioRxiv a license to display the preprint in perpetuity. It is made available under aCC-BY-NC-ND 4.0 International license.

309 (a) Labeled FynSensor Fyn (mCer-Fyn) kinase localizes to the plasma membrane as visualized 310 by Total internal reflection microscopy (TIRFM). U2OS cells were transiently transfected with 311 FynSensor (mCer-Fyn and myr-mVenus-F29, FRET donor and acceptor) and TIRFM imaging 312 performed. Shown here TIRF micrographs confirming plasma membrane localization of 313 labeled mCer-Fyn. (b) Labeled Fyn localization is not effected by expression of binder/FRET 314 acceptor (myr-mVenus-F29). Shown here are confocal micrographs depicting labeled Fyn localization with or without labeled F29 binder. U2OS cells were transiently transfected with either FynSensor (mCer-Fyn and myr-mVenus-F29, FRET donor and acceptor) or mCer-Fyn alone. Micrographs confirm that co-expression of binder/FRET acceptor (myr-mVenus-F29) has no measurable effect on the localization pattern of labeled Fyn. (Scale bar $=10 \mu \mathrm{m}$ ). 
a
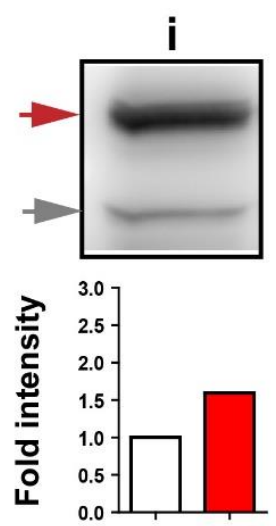

$u^{a^{0}} \mathrm{ce}^{e^{x^{x}}}$
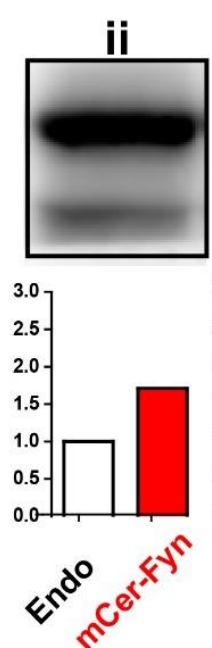
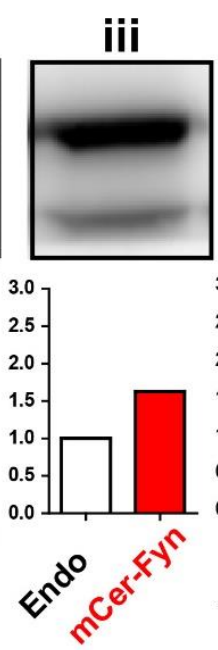
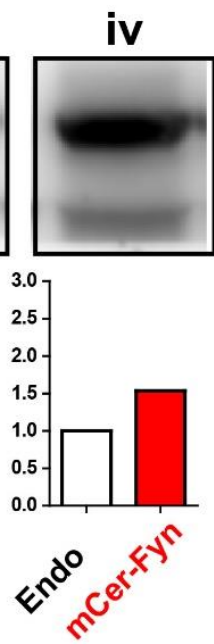

myr-

mVenus- mCer- Fyn-

b

myr-
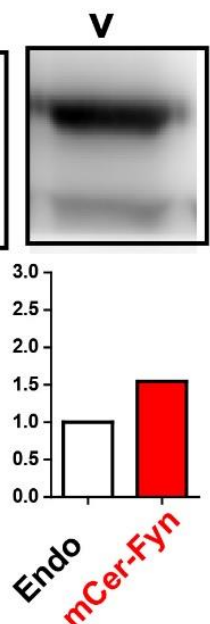

Fyn Sensor

mCer-

Fyn

Fyn

ERK1

ERK2

p-ERK1 $p$-ERK2

Vinculin 116

myr-mVen- 33

F29

myr-Ven- 29

C

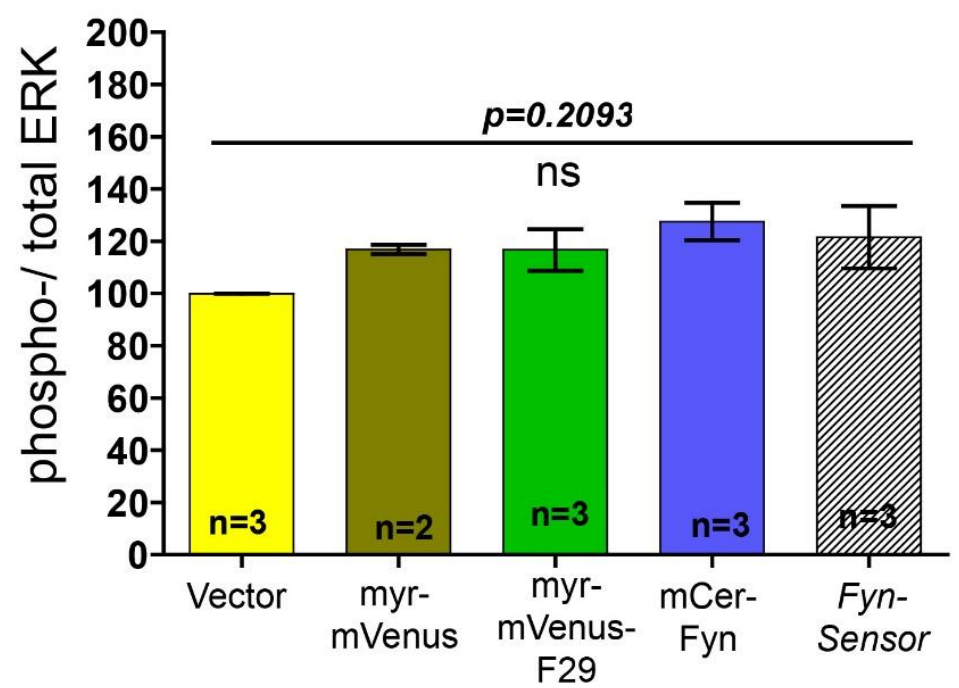




\section{Supplementary Fig. 14: FynSensor, labelled Fyn and F29 binder do not perturb downstream signalling in $\mathrm{C} 2 \mathrm{C} 12$ cells.}

322 Under expression levels (carefully quantified here) used for biosensor imaging in C2C12 cells,

323 the FynSensor labeled Fyn (mCer-Fyn) does not perturb downstream signalling as measured through ERK phosphorylation. (a)Immuno blot assay performed on cells transiently transfected with the FynSensor DNA constructs (same as used for biosensor imaging; donor: acceptor ratio, 1:2) and probed with Anti-Fyn antibody. Here five immunoblots (i-v) are shown where mCer-Fyn $(90 \mathrm{kDa})$ band has been marked with blue arrowhead and endogenous Fyn band is marked with grey arrowhead. For each case (i-v) the band intensity mCer-Fyn was compared with endogenous Fyn and plotted using GraphPad Prism and shown in a Box-andWhisker plot. Data from five blots (i-v) analysed using Student's unpaired one tailed t-test on

331 GraphPad Prism (respective p values are indicated on panel). Average from FIVE independent experiments show that mCer-Fyn is expressed at levels $(\sim 1.8$ fold $)$ comparable to the endogenous Fyn kinase. Imaging data in manuscript (see Fig. 7a, 8b, e) show that Fyn activity patterns revealed by FynSensor remain the same, independent of mCer-Fyn expression in this current expression range.

Expression of neither FynSensor (mCer-Fyn + myr-mVenus-F29), labelled Fyn (mCer-Fyn) nor labelled F29 (myr-mVenus-F29) alone perturb downstream signalling in C2C12 mytoblast cells, as measured through ERK phosphorylation levels. (b) Immunoblot showing levels of phosphorylated (anti-p-ERK) and total ERK (anti-ERK) in C2C12 cells expressing either FynSensor, labelled Fyn (mCer-Fyn), labelled F29 (myr-mVenus-F29), a myristoyl-taggedmVenus or a vector control. Also shown are levels of Fyn (anti-Fyn) and vinculin (antivinculin, control) as well as levels of myr-mVenus-F29 and myr-mVenus (anti-GFP). (c) Measurement of levels of ERK phosphorylation in cells expressing either FynSensor, labelled Fyn, labelled F29, a myristoyl-tagged-mVenus or a vector control. Graphs showing ratios of phospho-/total-ERK from immunoblot band densities from three independent experiments. No significant changes were seen in ERK phosphorylation levels. The pairs of means were tested using Newman-Keuls test in one-way ANOVA. The corresponding $p$ values are shown on 
a HEK239T(scrambled shRNA control)
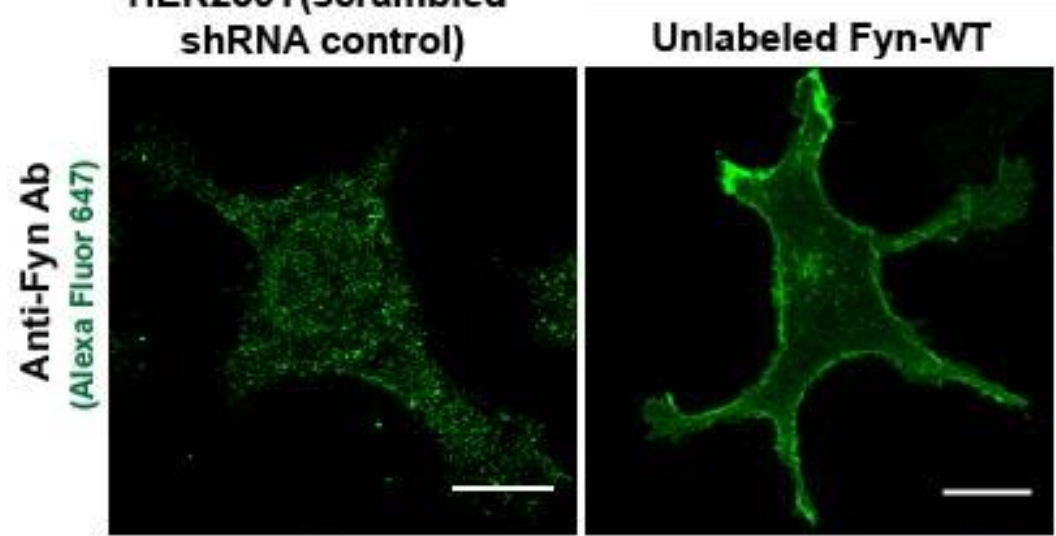

HEK293T-Fyn-KD cells
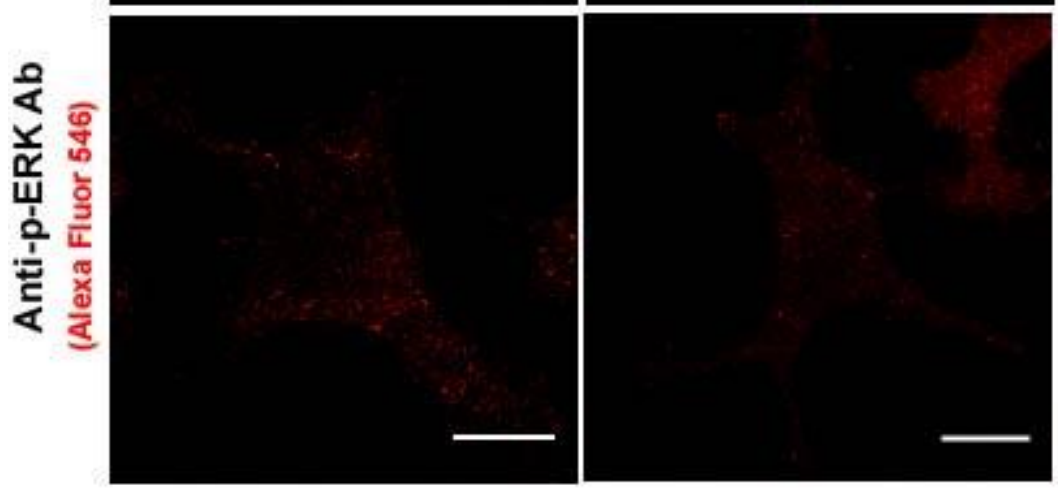

\section{FynSensor WT}
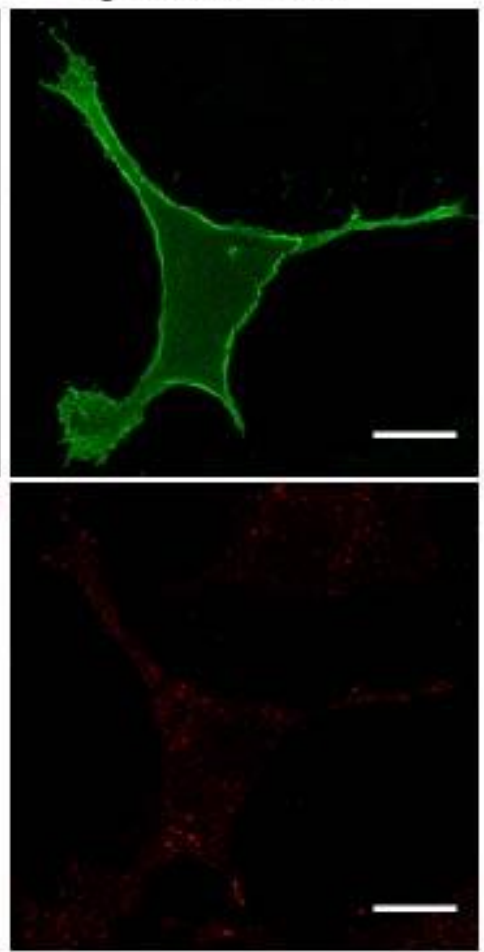

b

(i) Anti-Fyn Ab (Alexa Fluor 647)

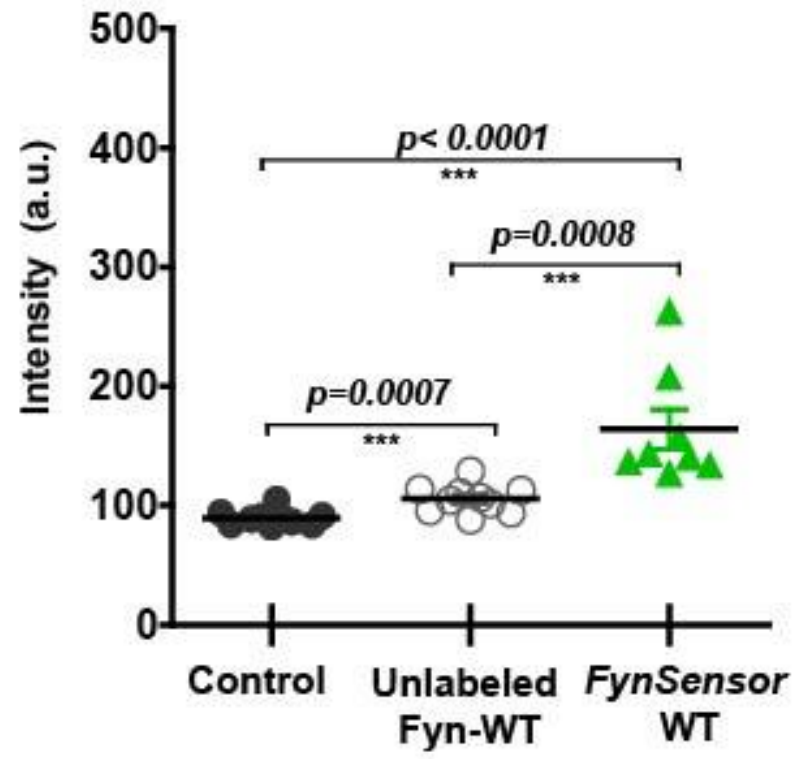

(ii) Anti-p-ERK Ab (Alexa Fluor 546)

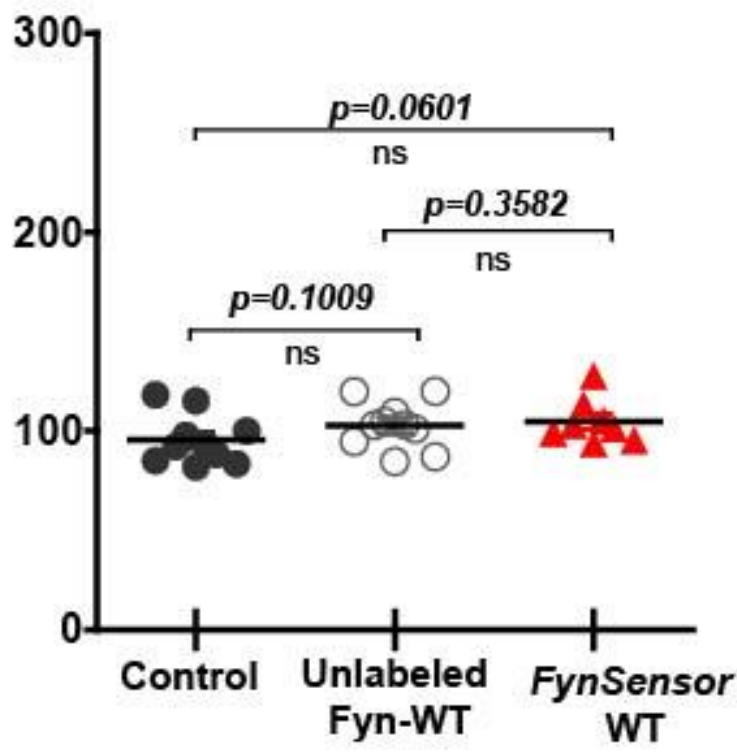

Supplementary Fig. 15: Ectopic expression of FynSensor, in HEK-293T cells used for imaging, does not perturb levels and intracellular patterns of ERK phosphorylation.

Levels and intracellular patterns of ERK phosphorylation were examined in knockdown-rescue cells expressing either FynSensor or wild-type, unlabeled Fyn and compared to control cells. Expression of labeled FynSensor Fyn (mCer-Fyn) in cells at levels and expression conditions used for biosensor imaging does not perturb downstream signaling ( $\mathrm{p}$-ERK levels and 
bioRxiv preprint doi: https://doi.org/10.1101/726133; this version posted August 5, 2019. The copyright holder for this preprint (which was not certified by peer review) is the author/funder, who has granted bioRxiv a license to display the preprint in perpetuity. It is made available under aCC-BY-NC-ND 4.0 International license.

localization). For FynSensor imaging, we have ectopically expressed labeled Fyn in HEK-293T cells where endogenous Fyn has been knocked down. This ensures that labeled Fyn levels are comparable to endogenous Fyn levels. In knock-down rescue cells, levels and patterns of pERK are comparable to control HEK293T cells. Further, the behaviour and localization of labeled FynSensor Fyn is similar to unlabeled, wild type Fyn.

(a) Immunofluorescence confocal micrographs showing Fyn expression/localization (anti-Fyn) as well as the levels and intracellular patterns of p-ERK (anti-phosphorylated-ERK) in either 'control cells' or Fyn KD HEK293T cells transiently transfected with either FynSensor (mCerFyn+ myr-mVen-F29) or unlabeled Fyn kinase (wt). Expression and plating conditions used are identical to conditions used for FynSensor imaging, see supplementary methods. Control cells are HEK-293T cells treated with endogenous Fyn levels (cells expressing scrambled shRNA HEK-293T and vector alone). Cells were co-stained with Anti-Fyn (mouse) and antiphospho-ERK primary antibody (rabbit) as well as Alexa Flour-647 conjugated anti-mouse and Alexa Flour-546 conjugated anti-rabbit secondary antibodies. Fyn kinase localization is shown on top panel (green LUT) while p-ERK localization pattern is shown (red LUT) in the panel below.

(b) Scatter-plot showing the levels of (i) Fyn and (ii) phospho-ERK immunofluorescence complex from multiple single cells. Mean \pm s.e.m is denoted. Student's unpaired one-tailed ttest has been used to determine the p-value. (Scale bar=10 $\mu \mathrm{m}$ ). Overall, in Fyn-KD cells ectopically expressing either labeled FynSensor or wt, unlabeled Fyn, p-ERK levels are similar to control cells in conditions identical to those used for imaging. 


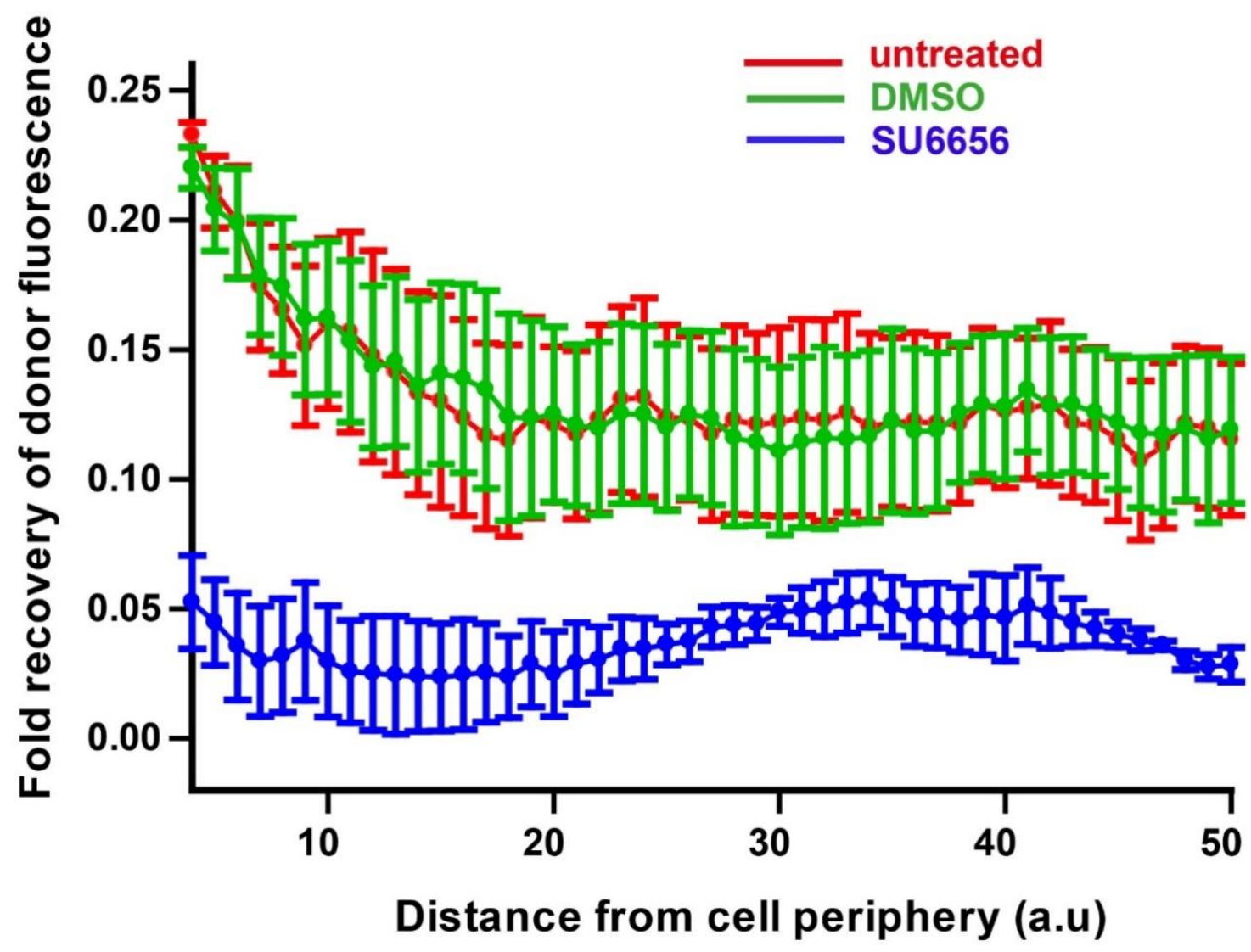

Supplementary Figure 16: FynSensor FRET readout is sensitive to kinase activity of Fyn.

FynSensor FRET signal is significantly attenuated when FynSensor expressing cells are treated with an inhibitor of Fyn (SFK) activity. Plotted here is the recovery of donor (mCer-Fyn) fluorescence (425-500 nm) when FynSensor expressing cells are subject to photobleaching at $515 \mathrm{~nm}$, corresponding to absorption wavelength of the acceptor (mVenus-F29 binder). The extent of this recovery is measured as a function of distance from cell periphery. Acceptorphotobleaching (APB) of FynSensor expressing U2OS cells causes a recovery in donor fluorescence showing clear FRET in live cells. This FRET signal (Fyn activity) is observed to be higher at the cell periphery. However, cells treated with $2 \mu \mathrm{M}$ of SU6656 inhibitor fail to show a similar pattern of recovery of donor fluorescence after ABP. Therefore, the FynSensor cellular FRET signal is significantly attenuated on inhibitor treatment. On the contrary, cells treated with DMSO (vehicle control) show an APB-induced recovery of donor fluorescence similar to untreated cells. Values are mean \pm s.e.m. 

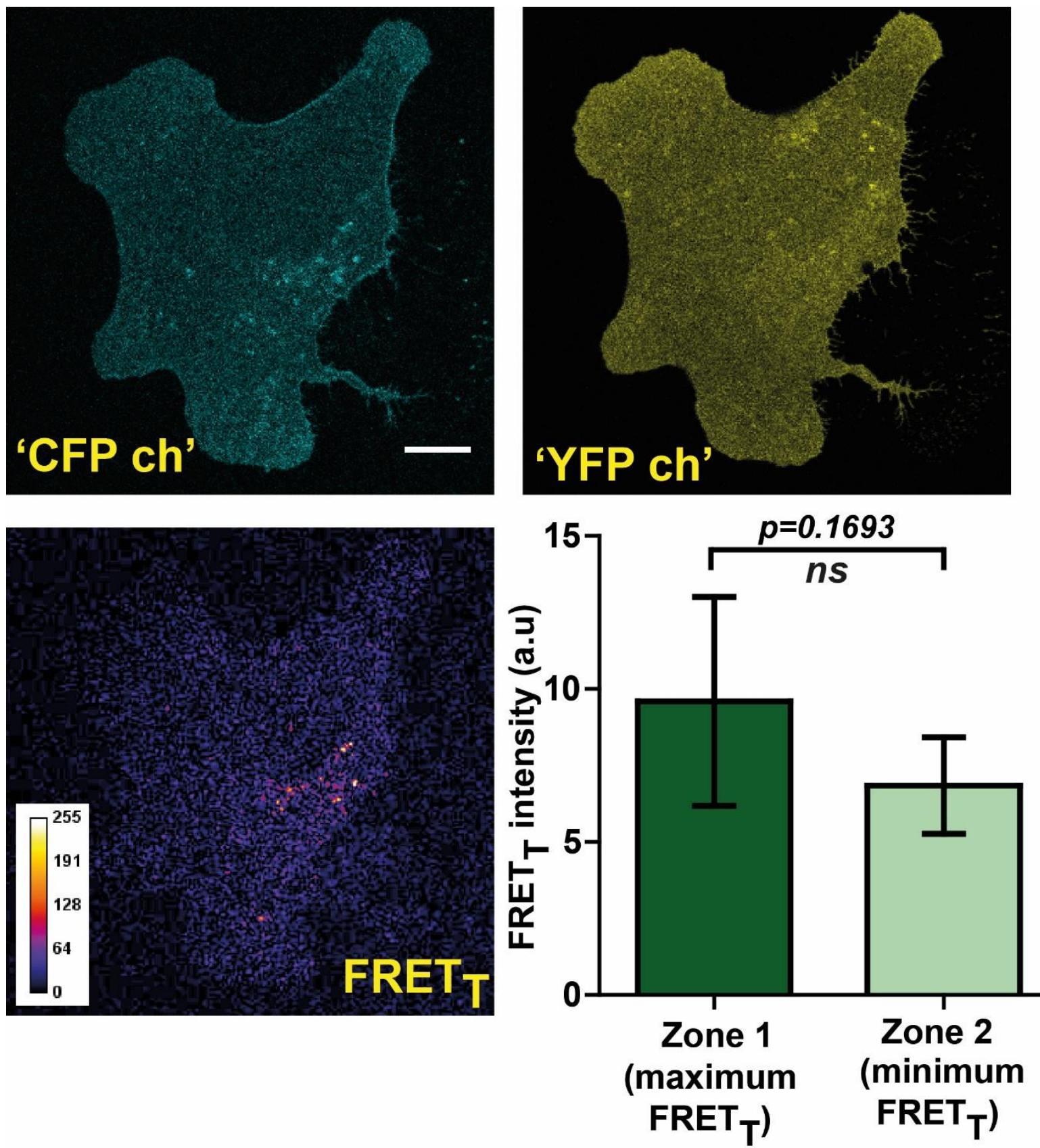

Supplementary Figure 17: FynSensor FRET readout is highly sensitive to F29 binding to Fyn kinase in cells. Non-binding mutant of FynSensor fails to show significant FRET response.

FynSensor FRET readout is dependent on F29 binding Fyn, with the non-binding control mutant of F29 (P41A) showing no or significantly reduced FRET $_{\mathrm{T}}$ signal. Confocal fluorescence micrographs showing Donor $_{\mathrm{ex}}$-Donor $_{\mathrm{em}}$, Acceptor $\mathrm{A}_{\mathrm{ex}}$-Aacceptor $_{\mathrm{em}}$ and corresponding FRET $_{\mathrm{T}}$ (sensitized emission) images for U2OS cells expressing mCer-Fyn AND non-binding control binder mVenus-F29 P41A (marked as 'CFP ch', 'YFP ch' and 'FRET ${ }_{\mathrm{T}}$ ' respectively). These images confirm that mCer-Fyn and non-binding control constructs are coexpressed in these cells and show expected 'CFP' and 'YFP' fluorescence on direct excitation. However, for non-binding control cells little or no FRET (sensitized emission) is seen. This 
bioRxiv preprint doi: https://doi.org/10.1101/726133; this version posted August 5, 2019. The copyright holder for this preprint (which was

not certified by peer review) is the author/funder, who has granted bioRxiv a license to display the preprint in perpetuity. It is made available under aCC-BY-NC-ND 4.0 International license.

403 lack of FRET shows that the FynSensor response is indeed specific and is dependent on F29

404 binding to activated Fyn. Also, plotted here is the average difference in $\mathrm{FRET}_{\mathrm{T}}$ levels between 405 intracellular zones showing maximum and minimum FRET $_{\mathrm{T}}$ (bottom right panel). Unlike the

406 cells expressing FynSensor, non-binder expressing U2OS cells do not show any discernible 407 spatially-compartmentalized kinase activity pattern. 
a

(i)

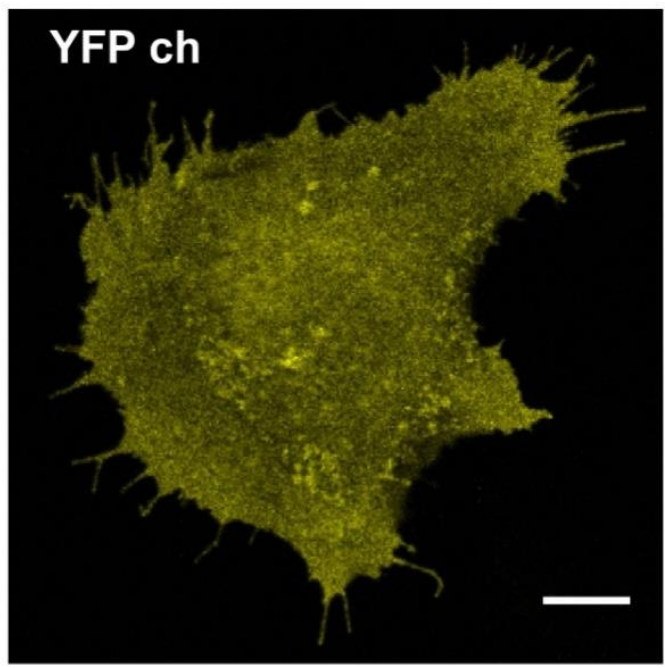

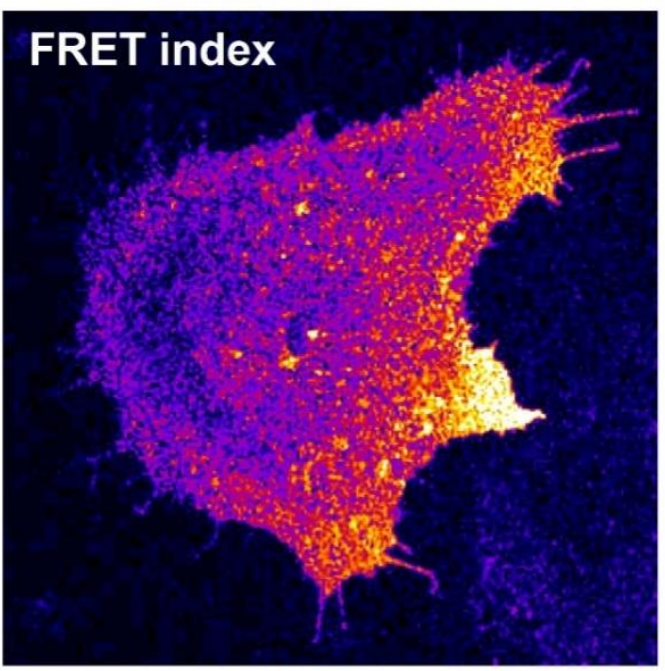

(ii)

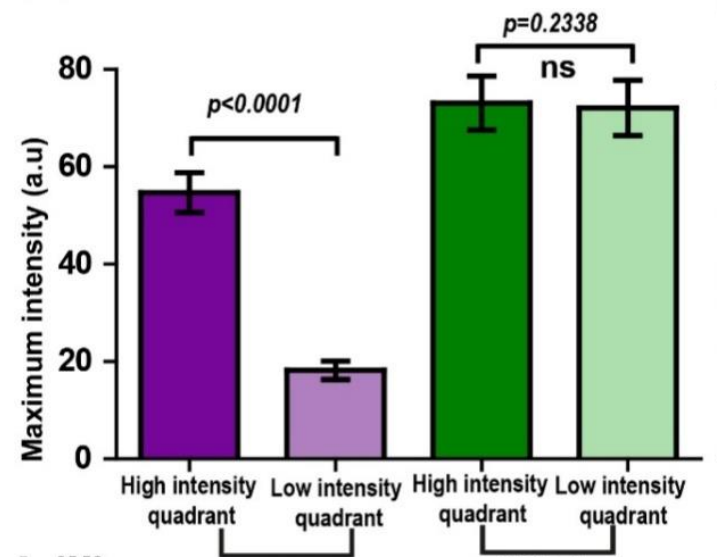

b(i)
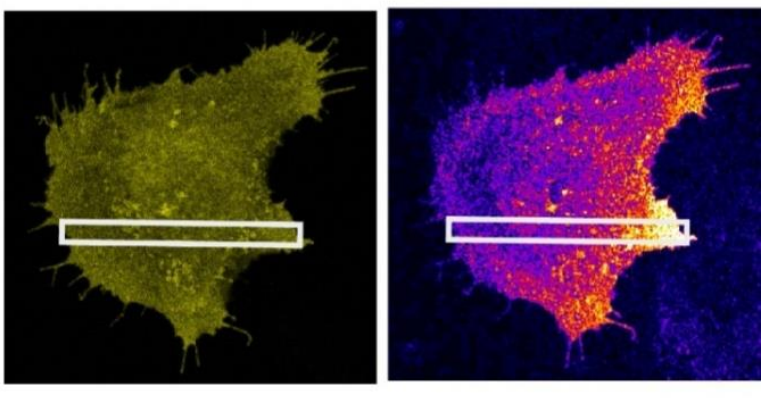

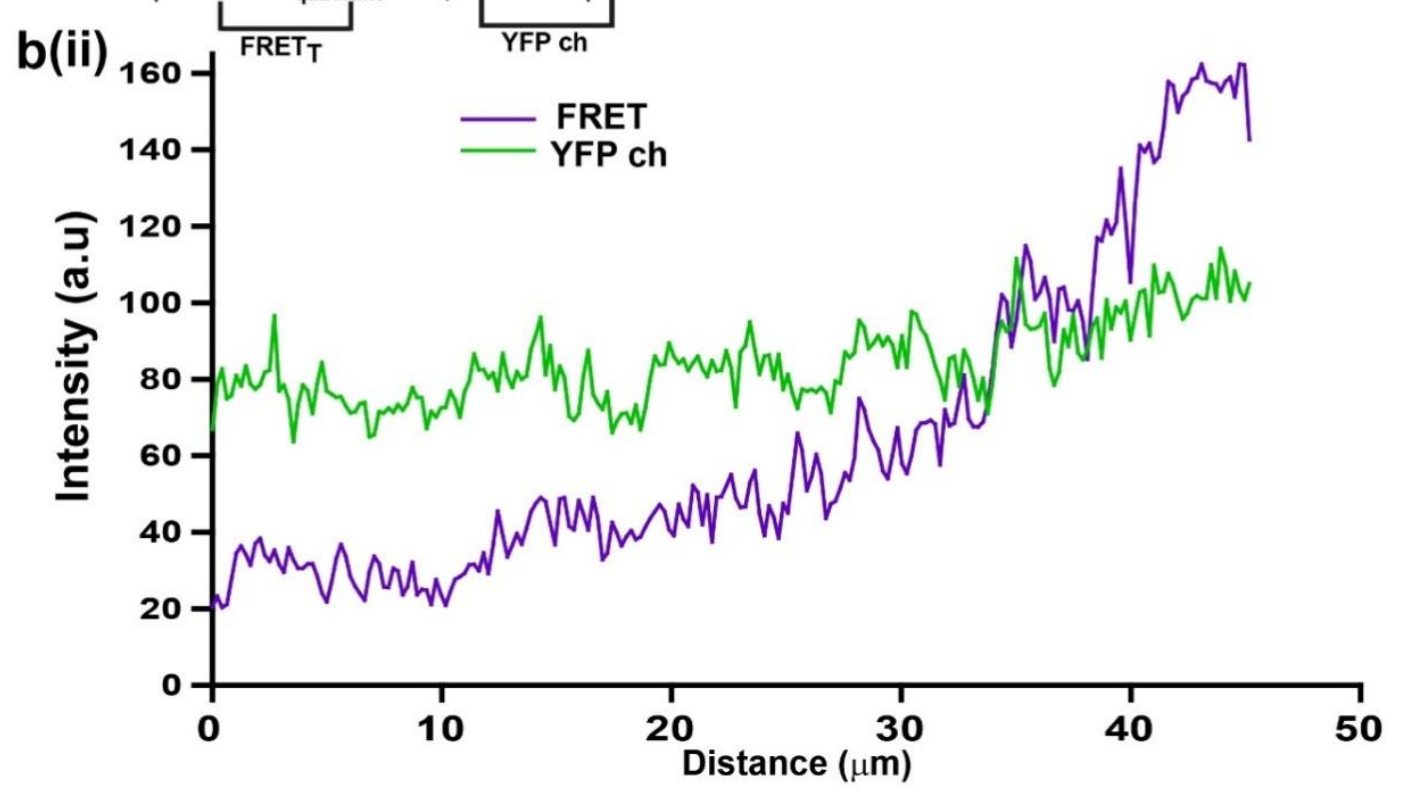

409 Supplementary Figure 18: Localized Fyn activity (spatially localized FynSensor FRET) 410 is not an artifact of binder localization.

411 (a) C2C12 cells showing localized Fyn activity (spatially localized FynSensor FRET), show a 412 spatially uniform binder localization. Therefore, localized FRET $_{\mathrm{T}}$ patterns are not determined 
bioRxiv preprint doi: https://doi.org/10.1101/726133; this version posted August 5, 2019. The copyright holder for this preprint (which was

not certified by peer review) is the author/funder, who has granted bioRxiv a license to display the preprint in perpetuity. It is made available under aCC-BY-NC-ND 4.0 International license.

413 by binder localization (i) Fluorescent confocal micrograph shows that while labeled F29 binder

414 (myr-mVenus-F29; 'YFP ch') localizes nearly uniformly in cells, the FynSensor FRET index

415 image clearly reveals a spatially-localized pattern of Fyn activity. (ii) Quantitative comparison

416 of average FRET $_{\mathrm{T}}$ and 'YFP ch' (labeled F29) intensities in two distinct intracellular zones

417 (quadrants). Quadrant analysis performed on both the YFP channel image and the FRET index

418 image for each cell (refer to methods section for details) yielded the maximum 'fluorescence

419 intensity' values from the 'high FRET $_{\mathrm{T}}$ quadrant (HFQ)' and the 'low FRET $\mathrm{T}$ quadrant (LFQ)'

420 in FRET index and 'YFP ch' images. Bar graph shows the average of these values from $\mathrm{n}=32$

421 cells. Student's paired one tailed-t-test was used to determine the p-value. While there is a clear

422 and significant difference in the $\mathrm{FRET}_{\mathrm{T}}$ levels across two intracellular zones (quadrants),

423 fluorescence due to the binder across the cell ('YFP ch') remains constant. (b) Illustration of

424 uniform binder localization in regions showing localized FRET $\mathrm{T}$. (i) Fluorescent intensity scan

425 across FRET and 'YFP ch' confocal micrographs to determine uniformity of signal. (ii) Plots

426 of average intensity values scanned across an extended cellular area (white box) for FRET

427 index and 'YFP ch'/binder alone images. While FRET $_{\mathrm{T}}$ is spatially modulated, binder

428 localization is uniform. 

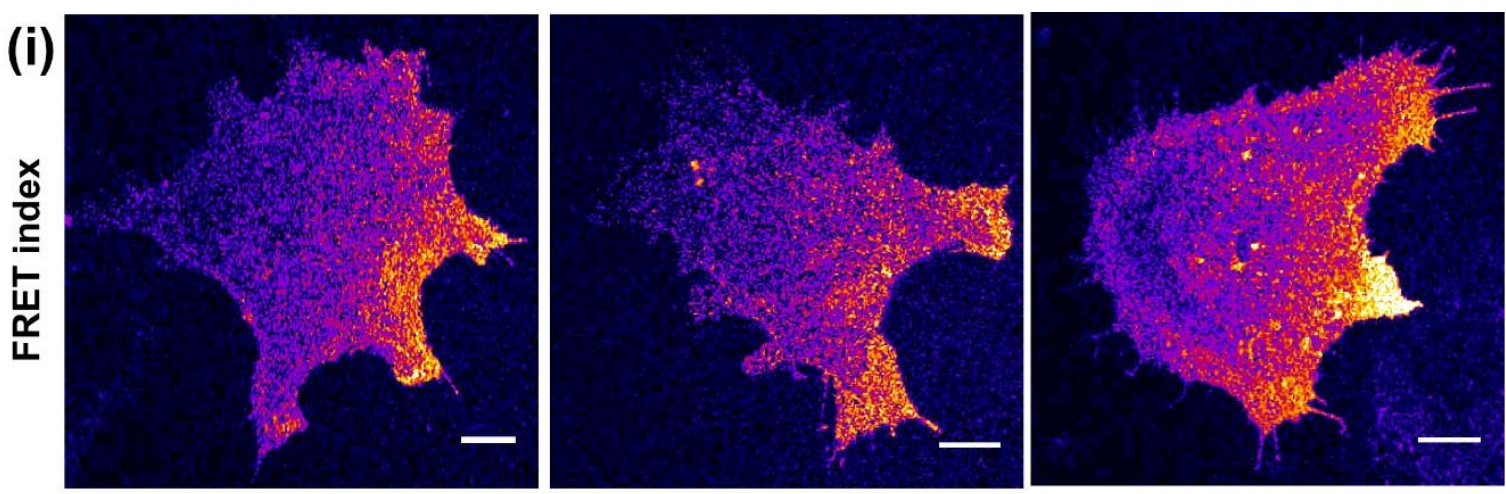

(ii)

b (i)
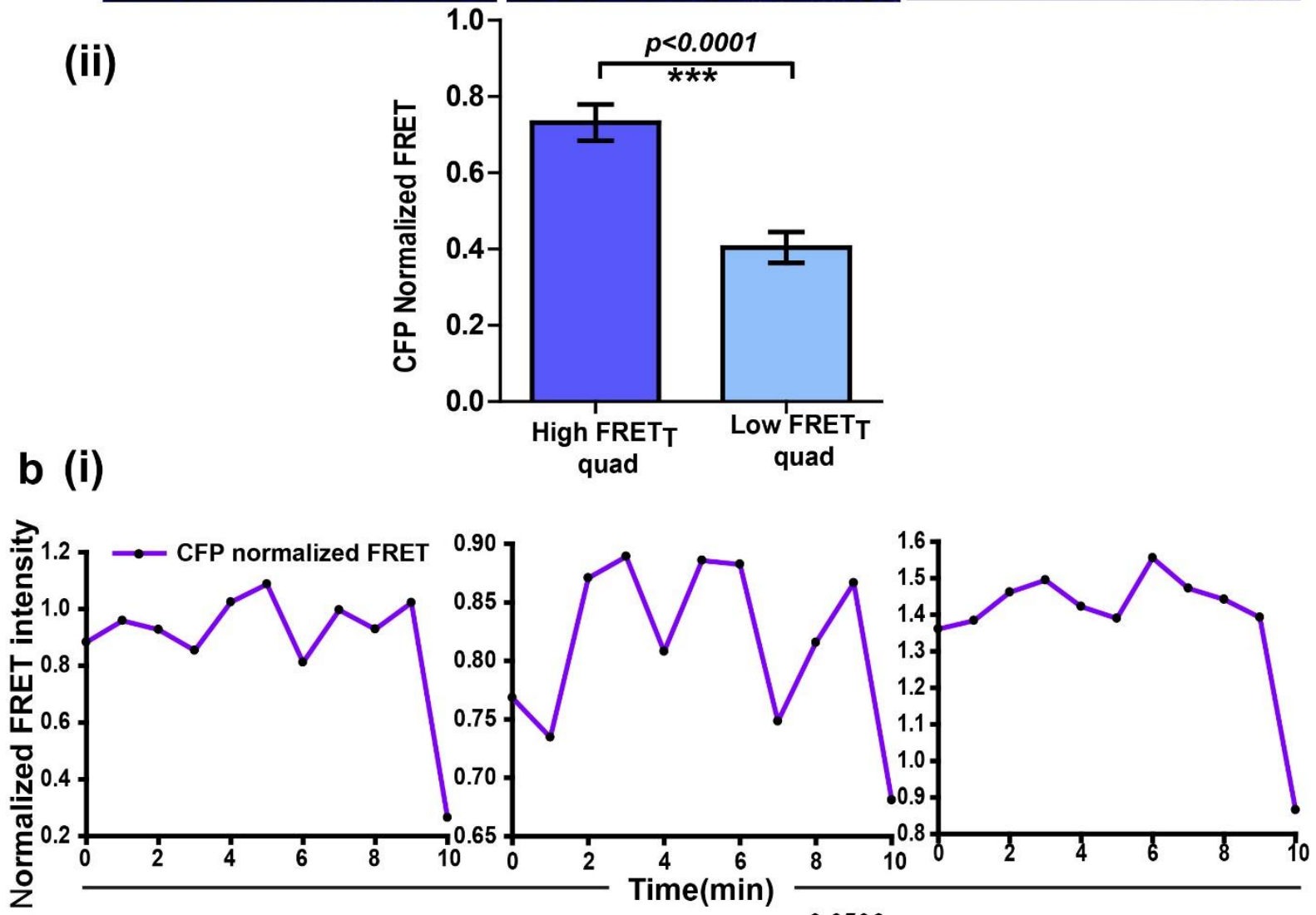

(ii)

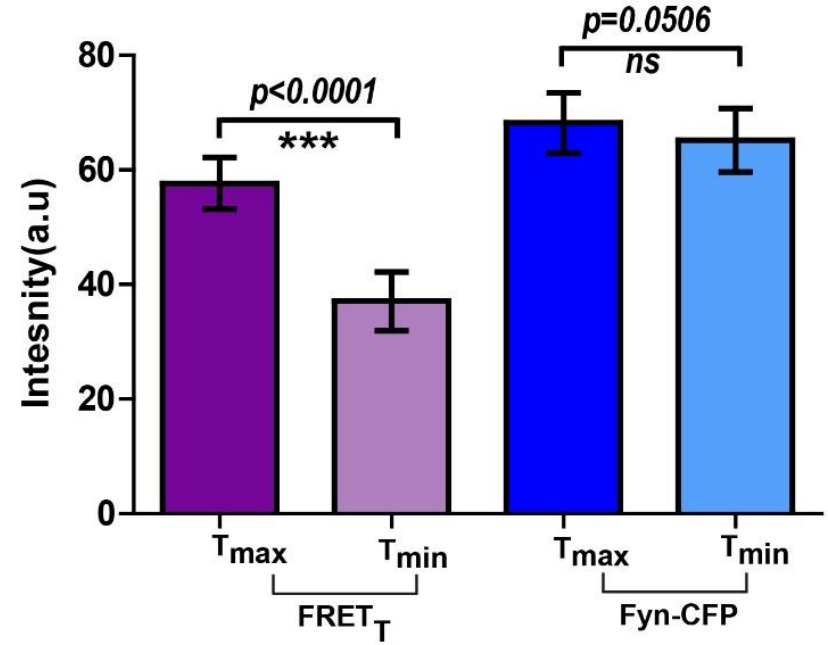

430 Supplementary Figure 19: Spatio-temporal modulations in Fyn activity are not simply due to changes in local Fyn protein concentration. 
bioRxiv preprint doi: https://doi.org/10.1101/726133; this version posted August 5, 2019. The copyright holder for this preprint (which was

not certified by peer review) is the author/funder, who has granted bioRxiv a license to display the preprint in perpetuity. It is made available under aCC-BY-NC-ND 4.0 International license.

432 (a) Spatially enhanced Fyn activity patterns are observed even when activation levels are normalized for any changes in local protein concentrations. This shows localized protein activation cannot simply be ascribed to increased protein accumulation. (i) FynSensor FRET index images (confocal micrographs) of 3 representative FynSensor expressing C2C12 cells showing localized Fyn activity. Specifically, intracellular zones with enhanced levels of active Fyn can be observed. (ii) Bar graphs comparing the mean of maximum 'CFP-normalized FRET' intensity values (see methods) in both the high FRET $_{\mathrm{T}}$ quadrant and low FRET $_{\mathrm{T}}$ quadrant in serum starved. For mean, 'CFP-normalized FRET' values were averaged across the specified quadrant in a single cell and then across cells. 'CFP normalized FRET' quantifies

441 Fyn activity normalized for changes in levels of labeled mCer-Fyn (protein concentration). For 442 each cell, time point showing max $\mathrm{FRET}_{\mathrm{T}}$ was chosen. Values are mean \pm s.e.m. Student's onetailed $\mathrm{t}$-test has been used to determine the $\mathrm{p}$-value $(\mathrm{n}=32$ cells

444 (b) Oscillations in Fyn activity oscillations are not due to fluctuations in local protein levels.

445 (i) Normalized FynSensor FRET temporal profiles (FRET ${ }_{\mathrm{T}}$ normalized to mCerulean 446 intensity/protein levels) also show the characteristic pulsatile signals as seen with FRET T. (ii) 447 While significant temporal oscillations are observed in Fyn activity, Fyn protein levels do not 448 fluctuate similarly. Shown here extent of oscillations in $\mathrm{FRET}_{\mathrm{T}}$ (cell averaged differences 449 between maximum and minimum FRET $_{\mathrm{T}}$ levels observed over time, in the high-FRET cellular 450 quadrant, HFQ). While significant changes in $\mathrm{FRET}_{\mathrm{T}}$ levels are observed, mCerulean ('CFP 451 ch') fluorescence in the corresponding cellular zones remains largely constant over time. ( $\mathrm{n}=8$ 452 cells). Values are mean \pm s.e.m. Student's paired one-tailed t-test has been used to calculate the 453 p-value. 
a

(i) FRET index-kinase activity
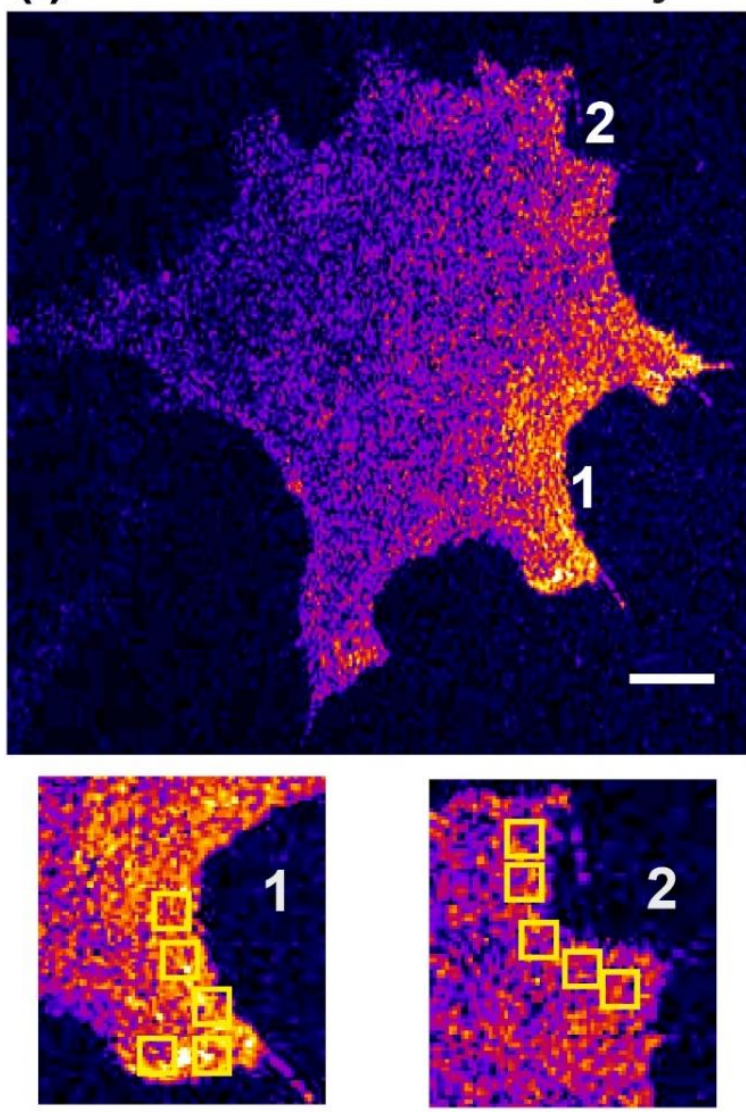

(ii) CFP ch-kinase localisation
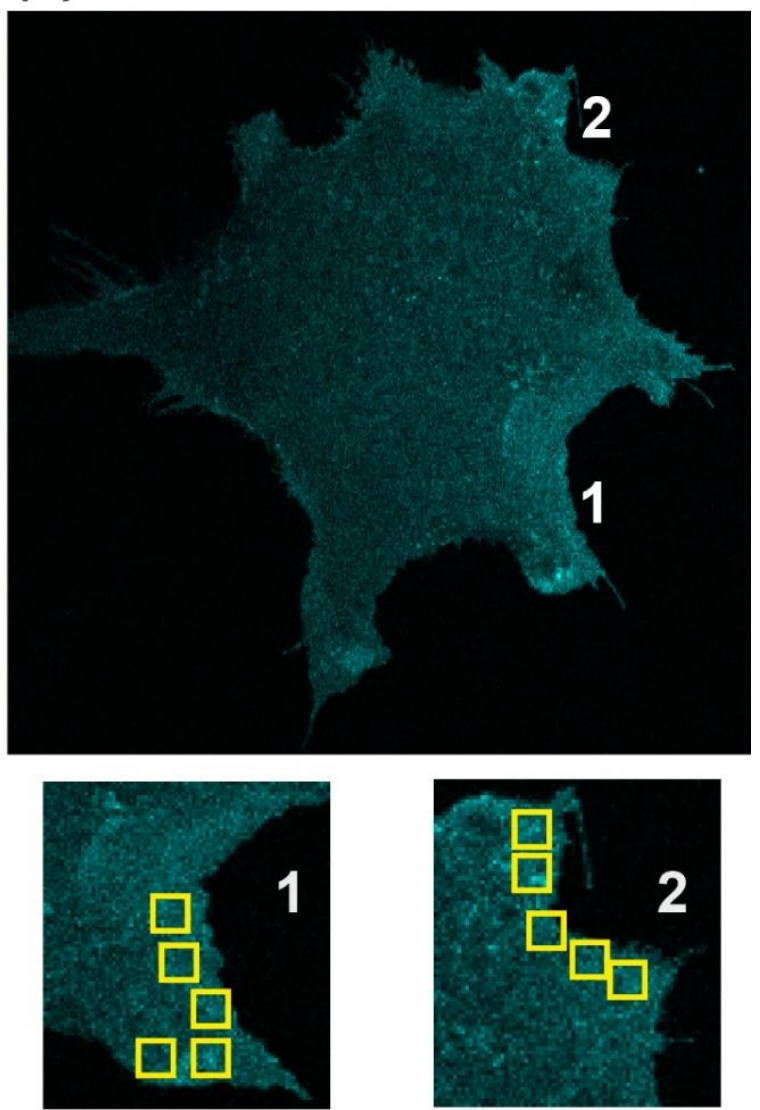

b

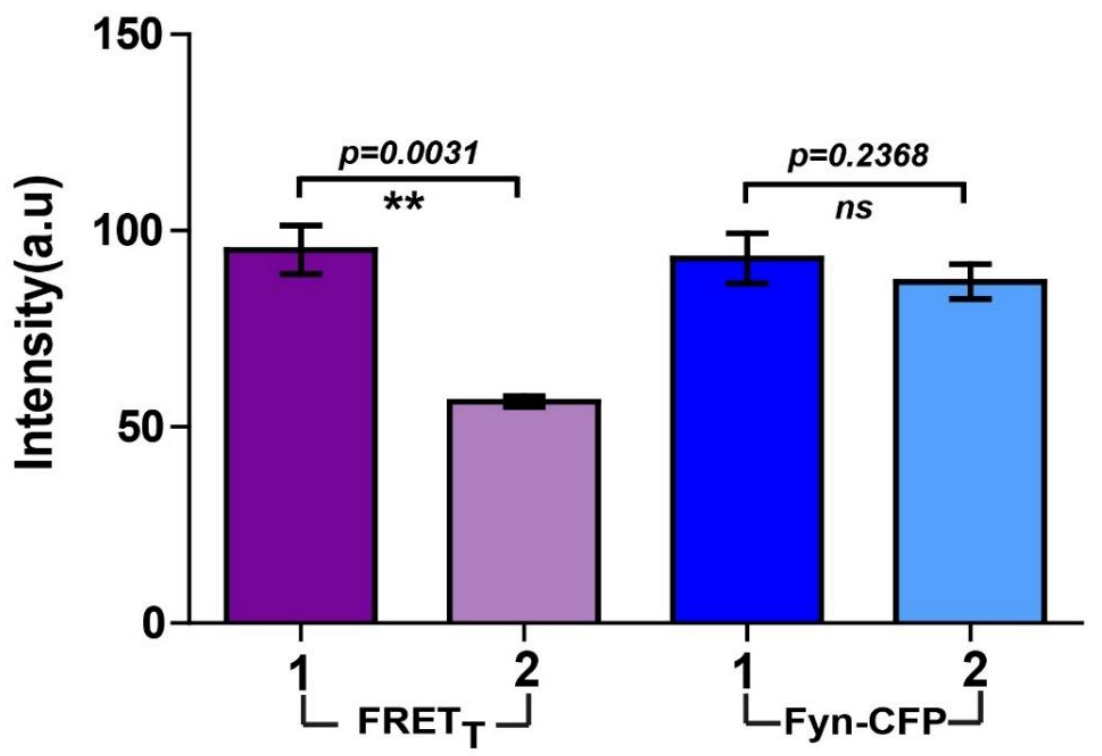

b

Supplementary Figure 20: Localized Fyn activity patterns are distinct from patterns of Fyn localization.

457 Fyn localization does not equate with Fyn activity. (a) Confocal micrograph of FynSensor 458 FRET index (Fyn activity, i) and 'CFP ch' (mCer-Fyn localization, ii) Images showing 459 intracellular zones (zones 1 and 2, enlarged and marked) with differing levels of FRET (Fyn 
bioRxiv preprint doi: https://doi.org/10.1101/726133; this version posted August 5, 2019. The copyright holder for this preprint (which was

not certified by peer review) is the author/funder, who has granted bioRxiv a license to display the preprint in perpetuity. It is made available under aCC-BY-NC-ND 4.0 International license.

460 activity) but similar levels of labeled Fyn protein. (b) Plots quantifying and comparing levels

461 of FRET $_{\mathrm{T}}$ (activity) and mCerulean fluorescence (' $\mathrm{CFP}$ ch'/protein concentration) between the 462 highlighted intracellular zones 1 and 2 (bar represent mean of the 5 ROIs drawn in each zone, 463 see a). Values are mean \pm s.e.m. Student's paired one-tailed t-test has been used to calculate the 464 p-value. Intracellular zones have significantly different FRET $_{\mathrm{T}}$ levels but similar local 465 concentrations of Fyn. 
bioRxiv preprint doi: https://doi.org/10.1101/726133; this version posted August 5, 2019. The copyright holder for this preprint (which was

not certified by peer review) is the author/funder, who has granted bioRxiv a license to display the preprint in perpetuity. It is made available under aCC-BY-NC-ND 4.0 International license.

a

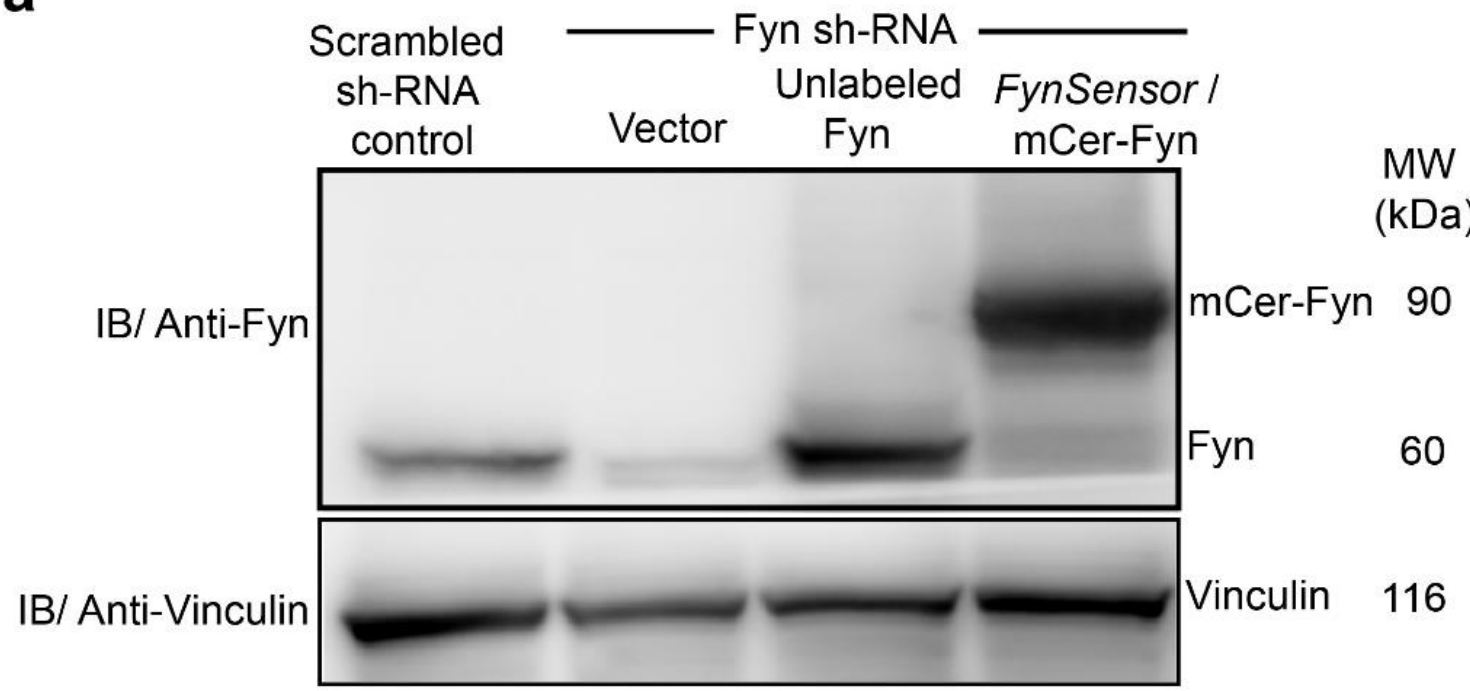

b

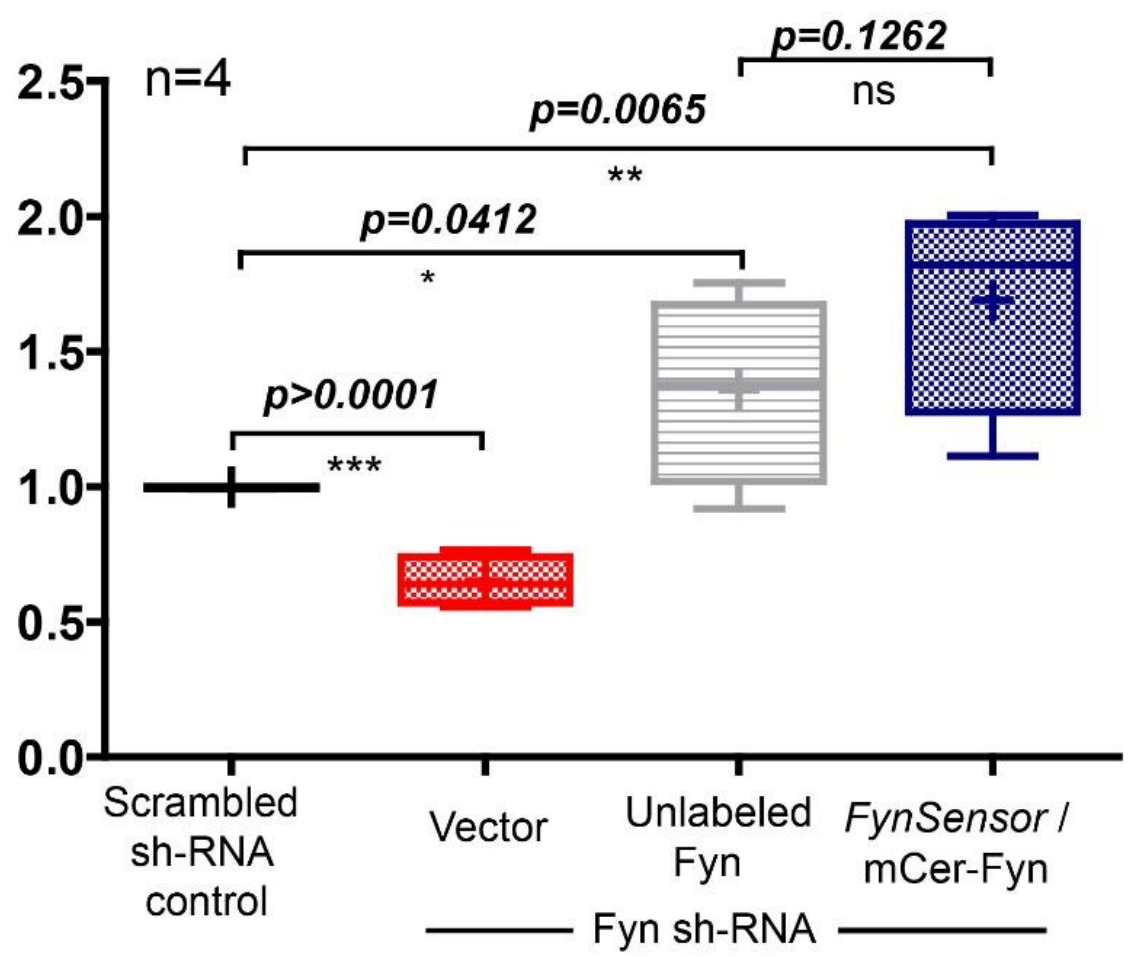

Supplementary Figure 21: Ectopic FynSensor expression in Fyn knockdown HEK-293T cells for biosensor imaging ensures labeled Fyn is expressed at levels comparable to endogenous Fyn.

(a) Immunoblot showing Fyn protein levels in either control HEK293T cells (harbouring scrambled sh RNA), Fyn knockdown cells (stable Fyn sh RNA cells along with an empty vector), Fyn knockdown cells expressing wild type, unlabeled Fyn or Fyn knockdown cells expressing FynSensor. Blot probed using Anti-Fyn antibody, with vinculin as a loading control. (b)The Box-and-Whisker plot quantifying Fyn protein levels in each of these cellular conditions from FOUR independent experiments. The band intensities as obtained using ImageJ software, were first normalized to their respective loading controls (vinculin protein) and then used for plotting relative Fyn protein levels by further normalizing sample values to 
bioRxiv preprint doi: https://doi.org/10.1101/726133; this version posted August 5, 2019. The copyright holder for this preprint (which was

not certified by peer review) is the author/funder, who has granted bioRxiv a license to display the preprint in perpetuity. It is made available under aCC-BY-NC-ND 4.0 International license.

478 levels of endogenous Fyn in control HEK293T cells. Plot confirms that knock-down cells 479 showed significant reduction in endogenous Fyn kinase levels. Expression of FynSensor 480 caused a recovery of total Fyn levels, with resulting protein levels comparable to levels of endogenous Fyn in control cells. Data was analyzed using Student's unpaired one tailed t-test on GraphPad Prism (respective p values are indicated on panel). 
a

b
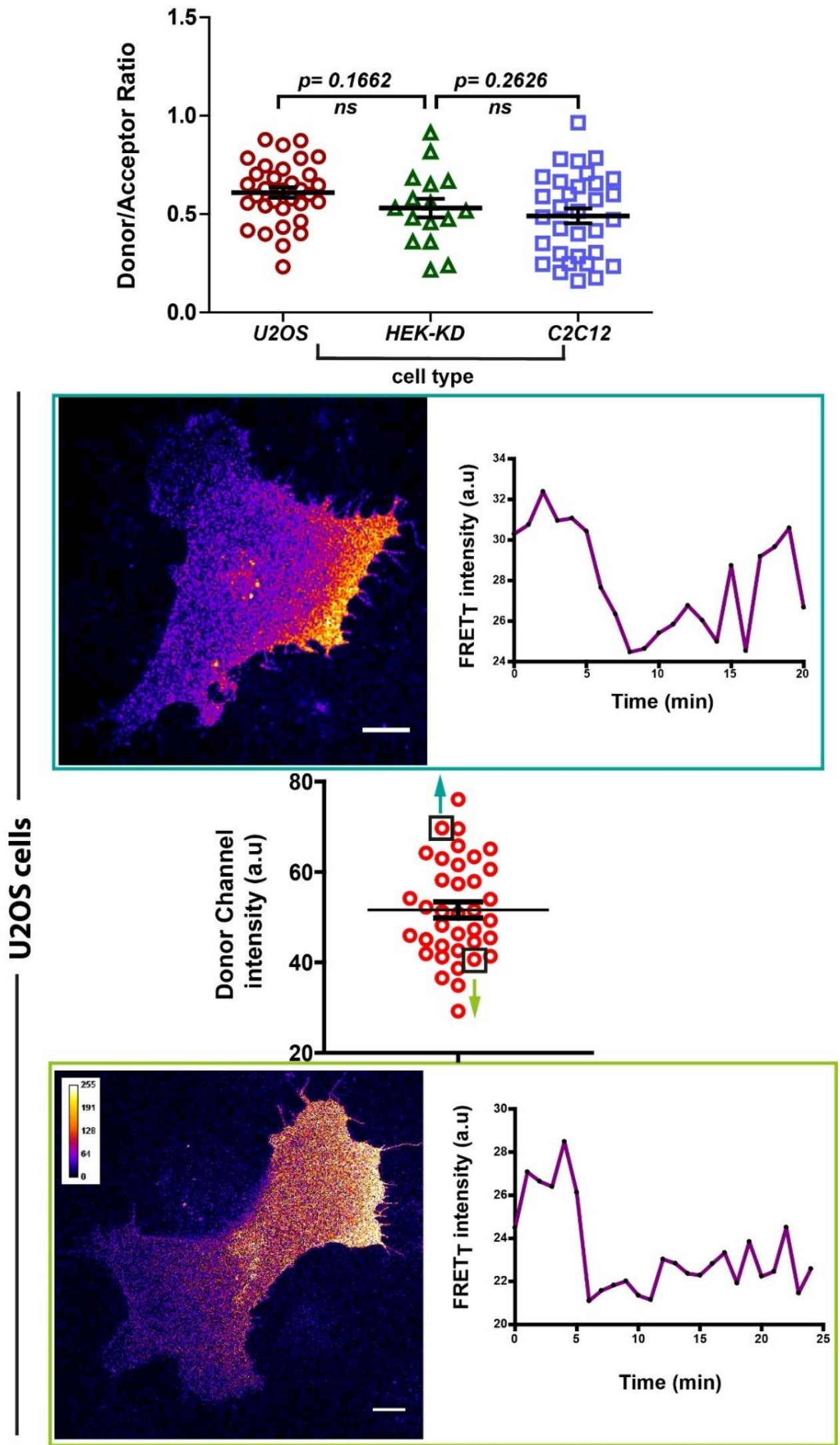

Supplementary Figure 22: FynSensor FRET spatial patterns and oscillations observed are independent of expression levels of labeled (FynSensor) Fyn. 
bioRxiv preprint doi: https://doi.org/10.1101/726133; this version posted August 5, 2019. The copyright holder for this preprint (which was not certified by peer review) is the author/funder, who has granted bioRxiv a license to display the preprint in perpetuity. It is made available under aCC-BY-NC-ND 4.0 International license.

FynSensor expression levels (as well as donor to acceptor ratios) in the distinct cell-types used for imaging are quantified here. Overall, the distributions across cell types are found to be similar. Also see Fig. 7c.(a) Scatter plot showing the distribution of donor/acceptor fluorescence ratios (mCer-Fyn/mVenus-F29) in the different cell types where FynSensor constructs have been ectopically expressing and used for biosensor imaging. Distributions of donor/acceptor rations are similar across cell types showing expression levels are carefully controlled and imaging conditions are comparable. (b) Consistent FynSensor spatio-temporal patterns are observed independent of precise levels of labeled Fyn. Confocal micrograph and time traces showing FRET patterns and temporal changes from representative U2OS cells from the data set, having either relatively 'high' (above the mean value of $51.61 \pm 1.805$ fluorescence intensity units, denoted by black box) or 'low' (below the mean value, black box) FynSensor expression (donor $\mathrm{m}$-Cer fluorescence intensities). Data and images confirm very similar spatially compartmentalized and pulsatile Fyn activity patterns from two example cells expressing different amounts of labeled Fyn. This demonstrates that the conserved Fyn activity patterns revealed here in individual cells are independent of precise expression levels, within the specified ranges. 